Alexandre de Macedo Torturela

\title{
Novos Métodos para Estimação por Mínimos Quadrados de Sistemas Esparsos
}

Tese apresentada como requisito parcial para obtenção do grau de Doutor pelo Programa de Pós-Graduação em Engenharia Elétrica do Departamento de Engenharia Elétrica da PUC-Rio

Orientador

Prof. Raimundo Sampaio Neto Co-Orientador: Prof. Cesar Augusto Medina Sotomayor 


\section{Alexandre de Macedo Torturela}

\section{Novos Métodos para Estimação por Mínimos Quadrados de Sistemas Esparsos}

Tese apresentada como requisito parcial para obtenção do grau de Doutor pelo Programa de Pós-Graduação em Engenharia Elétrica do Departamento de Engenharia Elétrica do Centro Técnico Científico da PUC-Rio. Aprovada pela Comissão Examinadora abaixo assinada.

Prof. Raimundo Sampaio Neto Orientador Centro de Estudos em Telecomunicações / PUC - Rio

Prof. Cesar Augusto Medina Sotomayor Co-Orientador Centro de Estudos em Telecomunicações / PUC - Rio

Prof. Marco Antonio Grivet Mattoso Maia Centro de Estudos em Telecomunicações / PUC - Rio

Prof. Rodrigo Caiado de Lamare Centro de Estudos em Telecomunicações / PUC - Rio

Prof. Ernesto Leite Pinto IME

Prof. Moisés Vidal Ribeiro UFJF

Prof. Juraci Ferreira Galdino IME

Prof. José Eugenio Leal Coordenador Setorial do Centro Técnico Científico - PUC-Rio 
Todos os direitos reservados. É proibida a reprodução total ou parcial do trabalho sem autorização da universidade, do autor e do orientador.

\section{Alexandre de Macedo Torturela}

Graduado em Engenharia de Comunicações pelo Instituto Militar de Engenharia em 1994. Pós-graduado em Análise, Gerência e Projeto de Sistemas pela Pontifícia Universidade Católica do Rio de Janeiro em 1997. Mestre em Engenharia Elétrica pelo Instituto Militar de Engenharia em 2002. Como oficial integrante do Quadro de Engenheiros Militares do Exército Brasileiro, trabalhou na Fábrica de Material de Comunicações e Eletrônica da Indústria de Material Bélico do Brasil entre os anos de 1995 e 2009 e, desde então, trabalha no Centro Tecnológico do Exército (CTEx), como integrante do Projeto Rádio Definido por Software (RDS) de Defesa, no âmbito do Ministério da Defesa.

Ficha Catalográfica

Torturela, Alexandre de Macedo

Novos Métodos para Estimação por Mínimos Quadrados de Sistemas Esparsos / Alexandre de Macedo Torturela; orientador: Raimundo Sampaio Neto; co-orientador: Cesar Augusto Medina Sotomayor. - 2014.

144 f. : il. (color.); $30 \mathrm{~cm}$

Tese (doutorado) - Pontifícia Universidade Católica do Rio de Janeiro, Departamento de Engenharia Elétrica, 2014. Inclui bibliografia

1. Engenharia Elétrica - Teses. 2. Estimação de sistemas esparsos. 3. Identificação de sistemas esparsos. 4. Estimação de canais esparsos. 5. Otimização convexa. 6. Compressive sensing. 7. Transmissão digital. 8. Ultra WideBand (UWB). 9. Impulse radio (IR). I. Sampaio Neto, Raimundo. II. Sotomayor, Cesar Augusto Medina. III. Pontifícia Universidade Católica do Rio de Janeiro. Departamento de Engenharia Elétrica. IV. Título. 
Aos meus pais Angela e Moacir. 


\section{Agradecimentos}

Aos professores Raimundo Sampaio Neto e César A. Medina, pela inestimável orientação acadêmica e por toda a demonstração de amizade.

À minha esposa Claudia, por todo o carinho e compreensão nos momentos de ausência, dedicados aos estudos e à pesquisa.

Aos meus familiares, por todo o incentivo e apoio cotidianos.

Aos meus superiores e companheiros de trabalho no Exército Brasileiro, que respectivamente me deram a oportunidade e o respaldo para que eu fizesse um curso de tão longa duração.

Aos meus colegas de pós-graduação, pela troca enriquecedora de conhecimentos e pela amizade.

Aos profissionais do CETUC e do Departamento de Engenharia Elétrica, por todo o apoio administrativo.

À PUC - Rio, por me conceder isenção das mensalidades, viabilizando assim eu cursar Doutorado em Engenharia Elétrica nesta tão conceituada Instituição de Ensino e Pesquisa.

Especiais agradecimentos ao professor Rodrigo de Lamare, cuja orientação e injeção de ânimo foram fundamentais para que eu lograsse êxito nessa longa empreitada. 


\section{Resumo}

Torturela, Alexandre de Macedo; Sampaio Neto, Raimundo; Sotomayor, Cesar Augusto Medina. Novos Métodos para Estimação por Mínimos Quadrados de Sistemas Esparsos. Rio de Janeiro, 2014. 144p. Tese de Doutorado - Departamento de Engenharia Elétrica, Pontifícia Universidade Católica do Rio de Janeiro.

Neste trabalho, quatro métodos projetados especificamente para a estimação de sistemas esparsos são originalmente elaborados e apresentados. São eles: Encolhimentos Sucessivos, Expansões Sucessivas, Minimização da Norma $l_{1}$ e Ajuste Automático do fator de regularização do Custo LS. Os quatro métodos propostos baseiam-se na técnica de estimação de sistemas lineares e invariantes no tempo pelo critério dos mínimos quadrados, universalmente conhecida por sua denominação em inglês - Least Squares (LS) Estimation, e incorporam técnicas relacionadas a otimização convexa e à teoria de compressive sensing. Os resultados obtidos em simulações mostram que os métodos em questão têm desempenho superior que a estimação LS convencional e que o algoritmo Recursive Least Squares (RLS) com regularização convexa denominado $l_{1}$-RLS, em muitos casos alcançando o desempenho ótimo apresentado pelo método de estimação LS Oráculo, no qual o suporte da resposta ao impulso em tempo discreto do sistema estimado é conhecido a priori. Além disso, os métodos propostos apresentam custo computacional menor que do algoritmo $l_{1}$-RLS.

\section{Palavras-chave}

Estimação de sistemas esparsos; Identificação de sistemas esparsos; Estimação de canais esparsos; Otimização convexa; Compressive sensing; Transmissão digital; Ultra WideBand (UWB); Impulse radio (IR). 


\section{Abstract}

Torturela, Alexandre de Macedo; Sampaio Neto, Raimundo (Advisor); Sotomayor, Cesar Augusto Medina (Co-advisor). Novel Sparse Systems Least Squares Estimation methods. Rio de Janeiro, 2014. 144p. PhD Thesis - Departamento de Engenharia Elétrica, Pontifícia Universidade Católica do Rio de Janeiro.

In this thesis, four methods specifically designed for sparse systems estimation are originally developed and presented, which were called here: Relaxations method, Successive Expansions method, $l_{1}$-norm Minimization method and Automatic Adjustment of the Regularization Factor method. The four proposed methods are based on the Least Squares (LS) Estimation method and incorporate techniques related to convex optimization and to the theory of compressive sensing. The simulation results show that the proposed methods herein present superior performance than the ordinary LS estimation method and the Recursive Least Squares (RLS) with convex regularization method $\left(l_{1}\right.$-RLS), in many cases achieving the same optimal performance presented by the LS Oracle method. Furthermore, the proposed methods demand lower computational cost than the $l_{1}$-RLS method.

\section{Keywords}

Sparse system identification; Sparse system estimation; Sparse channel estimation; Convex optimization; Compressive sensing; Digital transmission; Ultra WideBand (UWB); Impulse radio (IR). 


\section{Sumário}

1 Introdução $\quad 14$

1.1 Contextualização deste trabalho 14

1.2 Motivação e objetivo 16

$\begin{array}{lll}1.3 & \text { Organização do texto } & 17\end{array}$

$\begin{array}{lll}1.4 & \text { Notação adotada } & 18\end{array}$

2 Estimação LS de sistemas lineares e invariantes no tempo $\quad 19$

$\begin{array}{lll}2.1 & \text { Introdução } & 19\end{array}$

2.2 Esquema de Estimação LS 20

2.3 Processamento em blocos da estimação LS 22

2.4 Generalização do processamento em blocos da estimação LS 23

2.5 Considerações finais 24

3 Estimação Least Squares com suporte reduzido $\quad 25$

$\begin{array}{lll}3.1 & \text { Introdução } & 25\end{array}$

3.2 Apresentação do esquema de estimação LS com suporte reduzido 26

3.3 Composição da estimativa LS com suporte reduzido 28

3.4 Análise de convergência das estimativas LS com suporte reduzido 29

3.5 Análise de convergência do custo LS com suporte reduzido 32

3.6 O erro médio quadrático como medida de distância 35

3.7 Determinação da estimativa LS através de algoritmos de busca 36

3.7.1 Velocidade de convergência versus valor inicial da estimativa LS 39

3.8 Custo computacional 40

3.9 Considerações finais 42

4 Métodos propostos para estimação de sistemas esparsos 44

4.1 Introdução 44

4.2 O valor máximo admitido para o custo LS 45

4.2.1 Critério para escolha do fator de relaxamento do custo LS 46

4.3 Norma L1 como medida de esparsidade 47

$\begin{array}{lll}4.4 & \text { Estimação LS ótima } & 50\end{array}$

4.5 Método de Encolhimentos Sucessivos 51

4.5.1 Introdução $\quad 51$

4.5.2 Decimação iterativa $\quad 52$

4.5.3 Minimização linear $\quad 54$

4.5.4 Despolarização $\quad 55$

4.5.5 Algoritmo proposto $\quad 55$

4.5.6 Custo computacional $\quad 57$

4.5.7 Análise de desempenho $\quad 57$

4.6 Método de Expansões Sucessivas 64

4.6.1 Introdução 64

4.6.2 Algoritmo proposto 64

$\begin{array}{lll}\text { 4.6.3 Custo computacional } & 66\end{array}$

$\begin{array}{lll}\text { 4.6.4 Análise de desempenho } & 67\end{array}$ 
4.7 Método de Minimização da Norma L1 73

$\begin{array}{lll}4.7 .1 & \text { Introdução } & 73\end{array}$

$\begin{array}{lll}\text { 4.7.2 } & \text { Algoritmo proposto } & 74\end{array}$

$\begin{array}{lll}\text { 4.7.3 Análise de convergência } & 80\end{array}$

4.7.4 Interpretação geométrica $\quad 80$

4.7.5 Custo computacional 84

4.7.6 Avaliação de desempenho 84

4.8 Método de Regularização Automática do custo LS 90

$\begin{array}{lll}4.8 .1 & \text { Introdução } & 90\end{array}$

4.8.2 O valor ótimo do fator de regularização 90

4.8.3 Regularização do custo LS por homotopia 91

4.8.4 Variação do custo LS e da penalidade em função do fator de regularização $\quad 92$

4.8.5 Algoritmo proposto 94

4.8.6 Algoritmo de busca para obtenção da estimativa regularizada 95

4.8.7 Algoritmo de Regularização Automática em versão iterativa 96

$\begin{array}{ll}4.8 .8 \text { Custo computacional } & 100\end{array}$

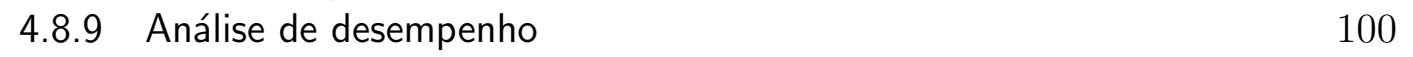

$\begin{array}{lll}4.9 & \text { Considerações finais } & 107\end{array}$

5 Avaliação de desempenho $\quad \mathbf{1 1 0}$

5.1 Introdução 110

5.2 Avaliação de desempenho dos métodos quando empregados na estimação de sistemas MESS 110

5.3 Avaliação de desempenho dos métodos quando empregados na estimação de canais IEEE 802.15.4a 118

$\begin{array}{lll}5.4 & \text { Avaliação do custo computacional } & 118\end{array}$

$\begin{array}{ll}5.5 \text { Considerações finais } & 128\end{array}$

6 Conclusão $\quad 129$

A Representação de sinais em sistemas UWB $\quad 134$

A.1 Modelo de sinal em tempo contínuo 134

A.2 Modelo de sinal em tempo discreto 136

A.3 Regras de decisão de símbolos 138

A.4 Impacto do erro de estimação do canal na detecção 140

$\begin{array}{lll}\text { B O Sistema Esparso de Máxima Entropia } & 144\end{array}$ 


\section{Lista de figuras}

3.1 Convergência de estimativas LS de um sistema $\operatorname{MESS}(100,10)$, com $\mathcal{E}_{\text {chip }} / \sigma_{z}^{2}=5 \mathrm{~dB}, \mathrm{~N}=16$ e $\mathrm{Q}=100$, em função do quantidade $k$ de blocos processados

3.2 Curvas de convergência do custo LS para $\mathcal{E}_{\text {chip }} / \sigma_{z}^{2}=10 \mathrm{~dB}$ e $\mathrm{N}=16$, avaliada em $\mathrm{Q}=1000$ realizações de um sistema $\operatorname{MESS}(100,10)$

3.3 Monotonicidade de uma sequência $\left\{J_{k}\left(\mathbb{S}_{i}\right) \mid \mathbb{S}_{i} \supset \mathbb{S}_{i+1}\right\}$, em $k=100$, gerada aleatoriamente, referente a uma única realização de um sistema $\operatorname{MESS}(100,10)$, com $\mathcal{E}_{\text {chip }} / \sigma_{z}^{2}=10 \mathrm{~dB}$ e $\mathrm{N}=16$

3.4 Quantidade média de iterações do Algoritmo de Busca LS em função de $|\mathbb{S}|$ para $\mathrm{N}=8, \mathrm{k}=10, \mathcal{E}_{\text {chip }} / \sigma_{z}^{2}=0 \mathrm{~dB}$ e $\rho=10^{-4}$, em $\mathrm{Q}=1000$ realizações de um sistema $\operatorname{MESS}(201,15)$, nos seguintes casos: $(1)\left(\hat{\mathbf{h}}_{k}^{\mathbb{S}}\right)^{(0)}=\mathbf{0},(2)\left(\hat{\mathbf{h}}_{k}^{\mathbb{S}}\right)^{(0)}=\left(\mathbf{b}_{k}\right)_{\mathbb{S}},(3)\left(\hat{\mathbf{h}}_{k}^{\mathbb{S}}\right)^{(0)}=\mathbf{I}_{\mathbb{S}, \mathbb{L}} \hat{\mathbf{h}}_{k}^{\mathbb{L}}$

3.5 Tempo médio gasto para determinar a estimativa $\mathrm{LS} \hat{\mathbf{h}}_{k}^{\mathbb{S}}$ em função de $|\mathbb{S}|$, para $\mathrm{N}=8, \mathrm{k}=10, \mathcal{E}_{\text {chip }} / \sigma_{z}^{2}=0 \mathrm{~dB}, \rho=10^{-4}$, em $\mathrm{Q}=1000$ realizações de um sistema MESS(201,15), empregando os seguinte métodos: (1) cálculo direto através de 3-55; (2) cálculo através de 3-57, empregando-se a Decomposição de Cholesky de $\left(\mathbf{A}_{k}\right)_{\mathbb{S}, \mathbb{S}}$; (3) busca através do Algoritmo 3.2, com $\left(\hat{\mathbf{h}}_{k}^{\mathbb{S}}\right)^{(0)}=\mathbf{0}$; (4) busca através do Algoritmo 3.2, com $\left(\hat{\mathbf{h}}_{k}^{\mathbb{S}}\right)^{(0)}=\left(\mathbf{b}_{k}\right)_{\mathbb{S}} ;(5)$ busca através do Algoritmo 3.2, com $\left(\hat{\mathbf{h}}_{k}^{\mathbb{S}}\right)^{(0)}=\mathbf{I}_{\mathbb{S}, \mathbb{L}} \hat{\mathbf{h}}_{k}^{\mathbb{L}}$, calculando-se previamente $\hat{\mathbf{h}}_{k}^{\mathbb{L}}$ através de 3-56 (com $\mathbf{A}_{k}^{i n v}$ calculada previamente e armazenada em memória).

4.1 Variação da média e do desvio-padrão de $L_{0}(\mathbf{h})$ em função de $S$, estimadas em $Q=1000$ realizações independentes de um sistema $\operatorname{MESS}(100, S)$, para cada valor de $S=1,2, \ldots, 100$

4.2 Exemplo do funcionamento do Método de Encolhimentos Sucessivos relativa à estimação de uma realização de um sistema $\operatorname{MESS}(50,10)$, com $K=5, \operatorname{SINR}=3 \mathrm{~dB}, N=16$ e $\lambda=1,2$

4.3 Enc. Suc.: $\operatorname{SINR}=5 \mathrm{~dB}, \operatorname{MESS}(100,10), \lambda=1,05, \mathrm{~N}=16$ e $\mathrm{Q}=1000 \quad 58$

4.4 Enc. Suc.: $\operatorname{SINR}=10 \mathrm{~dB}, \operatorname{MESS}(100,10), \lambda=1,05, \mathrm{~N}=16$ e $\mathrm{Q}=100058$

4.5 Enc. Suc.: $\operatorname{SINR}=15 d B, \operatorname{MESS}(100,10), \lambda=1,05, N=16$ e $Q=100059$

4.6 Enc. Suc.: $\operatorname{SINR}=20 d B, \operatorname{MESS}(100,10), \lambda=1,05, N=16$ e $Q=100059$

4.7 Enc. Suc.: $\operatorname{SINR}=25 d B, \operatorname{MESS}(100,10), \lambda=1,05, N=16$ e $Q=1000 \quad 60$

4.8 Enc. Suc.: $\operatorname{SINR}=30 \mathrm{~dB}, \operatorname{MESS}(100,10), \lambda=1,05, \mathrm{~N}=16$ e $\mathrm{Q}=1000 \quad 60$

4.9 Enc. Suc.: $\operatorname{SINR}=5 \mathrm{~dB}, \mathrm{MESS}(100,20), \lambda=1,05, \mathrm{~N}=16$ e $\mathrm{Q}=1000 \quad 61$

4.10 Enc. Suc.: $\operatorname{SINR}=10 d B, \operatorname{MESS}(100,20), \lambda=1,05, N=16$ e $Q=1000 \quad 61$

4.11 Enc. Suc.: $\operatorname{SINR}=15 d B, \operatorname{MESS}(100,20), \lambda=1,05, N=16$ e $Q=100062$

4.12 Enc. Suc.: $\operatorname{SINR}=20 d B, \operatorname{MESS}(100,20), \lambda=1,05, N=16$ e $Q=100062$

4.13 Enc. Suc.: $\operatorname{SINR}=25 \mathrm{~dB}, \operatorname{MESS}(100,20), \lambda=1,05, \mathrm{~N}=16$ e $\mathrm{Q}=100063$

4.14 Enc. Suc.: $\operatorname{SINR}=30 d B, \operatorname{MESS}(100,20), \lambda=1,05, N=16$ e $Q=100063$ 
4.15 Exp. Suc.: $\operatorname{MESS}(100,10), \operatorname{SINR}=5 d B, \lambda=1,05, N=16$ e $Q=1000$

4.16 Exp. Suc.: MESS $(100,10)$, $\operatorname{SINR}=10 \mathrm{~dB}, \lambda=1,05, \mathrm{~N}=16$ e $\mathrm{Q}=100068$

4.17 Exp. Suc.: MESS $(100,10)$, $\operatorname{SINR}=15 d B, \lambda=1,05, N=16$ e $Q=100068$

4.18 Exp. Suc.: MESS $(100,10)$, $S I N R=20 d B, \lambda=1,05, N=16$ e $Q=100069$

4.19 Exp. Suc.: $\operatorname{MESS}(100,10), \operatorname{SINR}=25 d B, \lambda=1,05, N=16$ e $Q=1000 \quad 69$

4.20 Exp. Suc.: $\operatorname{MESS}(100,10), \operatorname{SINR}=30 \mathrm{~dB}, \lambda=1,05, \mathrm{~N}=16$ e $\mathrm{Q}=100070$

4.21 Exp. Suc.: MESS $(100,20), \operatorname{SINR}=5 \mathrm{~dB}, \lambda=1,05, \mathrm{~N}=16$ e $\mathrm{Q}=100070$

4.22 Exp. Suc.: MESS $(100,20)$, $\operatorname{SINR}=10 \mathrm{~dB}, \lambda=1,05, \mathrm{~N}=16$ e $\mathrm{Q}=100071$

4.23 Exp. Suc.: $\operatorname{MESS}(100,20)$, $S I N R=15 d B, \lambda=1,05, N=16$ e $Q=100071$

4.24 Exp. Suc.: MESS $(100,20), \operatorname{SINR}=20 \mathrm{~dB}, \lambda=1,05, \mathrm{~N}=16$ e $\mathrm{Q}=100072$

4.25 Exp. Suc.: MESS $(100,20), \operatorname{SINR}=25 \mathrm{~dB}, \lambda=1,05, \mathrm{~N}=16$ e $\mathrm{Q}=100072$

4.26 Exp. Suc.: $\operatorname{MESS}(100,20), \operatorname{SINR}=30 d B, \lambda=1,05, N=16$ e $Q=100073$

4.27 Transição entre os sub-espaços $\mathbb{V}^{(i-1)}$ e $\mathbb{V}^{(i)}$, com $\mathbb{V}^{(i)} \subset \mathbb{V}^{(i-1)}$. A curva azul representa o trajeto de máxima distância $l_{1}$, no subespaço $\mathbb{V}^{(i-1)}$, entre $\hat{\mathbf{h}}_{k}^{\mathbb{S}^{(i-1)}}\left(\delta_{u}=0\right)$ e o ponto de contato $\mathbf{z}$ com $\mathbb{V}^{(i)}\left(\delta_{u}=a^{(i-1)}\right)$. A curva vermelha representa o trajeto de máxima distância $l_{1}$, no sub-espaço $\mathbb{V}^{(i)}$, entre $\hat{\mathbf{h}}_{k}^{\mathbb{S}^{(i)}}\left(\delta_{u}=0\right)$ e $\mathbf{y}$ $\left(\delta_{u}=b\right)$, passando pelo ponto $\mathbf{x} \equiv \mathbf{z}\left(\delta_{u}=a^{(i-1)}\right)$.

4.28 Unicidade da estimativa referente à minimização da norma $l_{1}$, restrita ao custo LS menor que $J_{\max }$ : (a) $J_{k}^{\mathbb{L}}(\mathbf{0}) \leq J_{\max }$, (b) $J_{k}^{\mathbb{L}}(\mathbf{0})>J_{\max }$

4.29 Tangência do hiper-elipsoide com: (a) face do hiperpoliedro; (b) aresta do hiperpoliedro; (c) vértice do hiperpoliedro

4.30 Analogia do Método de Minimização da Norma $l_{1}$ com a redução do nível de água em uma represa. Neste exemplo $|\mathbb{L}|=20$, onde: (a) se $\delta_{u}=0$, então $J_{k}^{\mathbb{L}}\left(\tilde{\mathbf{h}}_{k}\right)=J_{k}(\mathbb{L})$; (b) se $\delta_{u}>0$, então $J_{k}^{\mathbb{L}}\left(\tilde{\mathbf{h}}_{k}\right)>J_{k}(\mathbb{L})$. À medida em que $\delta_{u}$ aumenta, $J_{k}^{\mathbb{L}}\left(\tilde{\mathbf{h}}_{k}\right)$ também aumenta, ao mesmo tempo em que os elementos de $\tilde{\mathbf{h}}_{k}$ são encolhidos. Dependendo do valor de $\delta_{u}$, alguns elementos de $\tilde{\mathbf{h}}_{k}$ podem ser zerados, o que é ilustrado em (b) pelas superfícies do fundo da represa acima do nível de água.

4.31 NMSD do custo LS para sistema MESS(100,S), com $S=10$ e $S=20, N=32, \lambda=1,01$ e $Q=1000$

4.32 Min. $l_{1}$ : $\operatorname{MESS}(100,10), \operatorname{SINR}=15 \mathrm{~dB}, \lambda=1,05, N=16$ e $\mathrm{Q}=1000$

4.33 Min. $l_{1}$ : $\operatorname{MESS}(100,10), \operatorname{SINR}=20 \mathrm{~dB}, \lambda=1,05, N=16$ e $\mathrm{Q}=1000$

4.34 Min. $l_{1}$ : $\operatorname{MESS}(100,10), \operatorname{SINR}=25 \mathrm{~dB}, \lambda=1,05, N=16$ e $\mathrm{Q}=1000$

4.35 Min. $l_{1}$ : $\operatorname{MESS}(100,10), \operatorname{SINR}=30 \mathrm{~dB}, \lambda=1,05, \mathrm{~N}=16$ e $\mathrm{Q}=1000 \quad 87$

4.36 Min. $l_{1}$ : $\operatorname{MESS}(100,20), \operatorname{SINR}=15 \mathrm{~dB}, \lambda=1,05, \mathrm{~N}=16$ e $\mathrm{Q}=1000 \quad 88$

4.37 Min. $l_{1}$ : MESS $(100,20), \operatorname{SINR}=20 \mathrm{~dB}, \lambda=1,05, \mathrm{~N}=16$ e $\mathrm{Q}=1000 \quad 88$

4.38 Min. $l_{1}$ : $\operatorname{MESS}(100,20), \operatorname{SINR}=25 \mathrm{~dB}, \lambda=1,05, N=16$ e $\mathrm{Q}=1000$

4.39 Min. $l_{1}$ : $\operatorname{MESS}(100,20), \operatorname{SINR}=30 \mathrm{~dB}, \lambda=1,05, N=16$ e $\mathrm{Q}=1000 \quad 89$

4.40 Curva de tradeoff entre $p(\rho)$ e $q(\rho)$ para uma única realização de um sistema $\operatorname{MESS}(100,10)$ e SINR $=10 \mathrm{~dB}$, com $N=32$ e $k=50 \quad 94$

4.41 Mecanismo de bisseção em uma única realização de canal gaussiano esparso com $L=100, S=10, \mathrm{SINR}=15 \mathrm{~dB}, N=32$ e $\lambda=1,05$. Parâmetros iniciais: $\check{\mathbf{h}}_{0}=\hat{\mathbf{h}}_{1}, \rho_{\max , 0}=1$ e $\rho_{\min , 0}=1 E-10$, $\rho_{0}=0,01$, tol $=0,01, \eta=0,0001, \beta=0,8$ e $\epsilon=0,001$ 
4.42 Variação média do fator de regularização $\rho$ ao longo da sequência de treinamento referente a $Q=1000$ realizações independentes de um sistema $\operatorname{MESS}(100, S)$, com $N=16$ e $\lambda=1,05$. Parâmetros iniciais: $\check{\mathbf{h}}_{0}=\hat{\mathbf{h}}_{1}, \rho_{\max , 0}=1$ e $\rho_{\min , 0}=1 E-10, \rho_{0}=0,5$, tol $=0,01, \eta=0,0001, \beta=0,8$ e $\epsilon=0,001$

4.43 Reg. iter.: MESS(100,10), $\operatorname{SINR}=5 \mathrm{~dB}, \lambda=1,05, N=16$ e $Q=1000101$

4.44 Reg. iter.: $\operatorname{MESS}(100,10), \operatorname{SINR}=10 \mathrm{~dB}, \lambda=1,05, \mathrm{~N}=16$ e $\mathrm{Q}=1000101$

4.45 Reg. iter.: $\operatorname{MESS}(100,10), \operatorname{SINR}=15 \mathrm{~dB}, \lambda=1,05, \mathrm{~N}=16$ e $\mathrm{Q}=1000102$

4.46 Reg. iter.: $\operatorname{MESS}(100,10), \operatorname{SINR}=20 \mathrm{~dB}, \lambda=1,05, \mathrm{~N}=16$ e $\mathrm{Q}=1000102$

4.47 Reg. iter.: $\operatorname{MESS}(100,10), \operatorname{SINR}=25 \mathrm{~dB}, \lambda=1,05, \mathrm{~N}=16$ e $\mathrm{Q}=1000103$

4.48 Reg. iter.: $\operatorname{MESS}(100,10), \operatorname{SINR}=30 \mathrm{~dB}, \lambda=1,05, \mathrm{~N}=16$ e $\mathrm{Q}=1000103$

4.49 Reg. iter.: $\operatorname{MESS}(100,20), \operatorname{SINR}=5 \mathrm{~dB}, \lambda=1,05, \mathrm{~N}=16$ e $\mathrm{Q}=1000104$

4.50 Reg. iter.: $\operatorname{MESS}(100,20), \quad \operatorname{SINR}=10 \mathrm{~dB}, \lambda=1,05, \quad \mathrm{~N}=16 \mathrm{e}$ $\mathrm{Q}=1000$

4.51 Reg. iter.: $\operatorname{MESS}(100,20), \quad \operatorname{SINR}=15 \mathrm{~dB}, \lambda=1,05, \quad \mathrm{~N}=16$ e $\mathrm{Q}=1000$

4.52 Reg. iter.: $\operatorname{MESS}(100,20), \quad \operatorname{SINR}=20 \mathrm{~dB}, \lambda=1,05, \quad \mathrm{~N}=16$ e $\mathrm{Q}=1000$

4.53 Reg. iter.: $\operatorname{MESS}(100,20), \quad \operatorname{SINR}=25 \mathrm{~dB}, \lambda=1,05, \quad \mathrm{~N}=16$ e $\mathrm{Q}=1000$

4.54 Reg. iter.: $\operatorname{MESS}(100,20), \quad \operatorname{SINR}=30 \mathrm{~dB}, \lambda=1,05, \quad \mathrm{~N}=16$ e $\mathrm{Q}=1000$

5.1 Comparação de desempenho: $\operatorname{MESS}(100,10), \operatorname{SINR}=5 \mathrm{~dB}, \lambda=$ $1,05, N=16$ e $Q=1000$

5.2 Comparação de desempenho: $\operatorname{MESS}(100,10), \operatorname{SINR}=10 \mathrm{~dB}$, $\lambda=1,05, N=16$ e $Q=1000$

5.3 Comparação de desempenho: $\operatorname{MESS}(100,10), \operatorname{SINR}=15 \mathrm{~dB}$, $\lambda=1,05, N=16$ e $Q=1000$

5.4 Comparação de desempenho: $\operatorname{MESS}(100,10), \operatorname{SINR}=20 \mathrm{~dB}$, $\lambda=1,05, N=16$ e $Q=1000$

5.5 Comparação de desempenho: $\operatorname{MESS}(100,10), \operatorname{SINR}=25 \mathrm{~dB}$, $\lambda=1,05, N=16$ e $Q=1000$

5.6 Comparação de desempenho: $\operatorname{MESS}(100,10)$, SINR $=30 \mathrm{~dB}$, $\lambda=1,05, N=16$ e $Q=1000$

5.7 Comparação de desempenho: $\operatorname{MESS}(100,20), \operatorname{SINR}=5 \mathrm{~dB}, \lambda=$ $1,05, N=16$ e $Q=1000$

5.8 Comparação de desempenho: $\operatorname{MESS}(100,20), \operatorname{SINR}=10 \mathrm{~dB}$, $\lambda=1,05, N=16$ e $Q=1000$

5.9 Comparação de desempenho: $\operatorname{MESS}(100,20), \operatorname{SINR}=15 \mathrm{~dB}$, $\lambda=1,05, N=16$ e $Q=1000$

5.10 Comparação de desempenho: $\operatorname{MESS}(100,20), \operatorname{SINR}=20 \mathrm{~dB}$, $\lambda=1,05, N=16$ e $Q=1000$

5.11 Comparação de desempenho: $\operatorname{MESS}(100,20), \operatorname{SINR}=25 \mathrm{~dB}$, $\lambda=1,05, N=16$ e $Q=1000$

5.12 Comparação de desempenho: $\operatorname{MESS}(100,20), \operatorname{SINR}=30 \mathrm{~dB}$, $\lambda=1,05, N=16$ e $Q=1000$ 
5.13 Comparação de desempenho entre os métodos de Encolhimentos Sucessivos, Expansões Sucessivas despolarizada, Minimização da norma $l_{1}$ despolarizada, Ajuste automático do fator de regularização do custo LS, LS Full-support, LS Oráculo e $l_{1}$-RLS, com: $\operatorname{MESS}(64,8), \operatorname{SINR}=20 \mathrm{~dB}, \lambda=1,2, N=1000, M=1 \mathrm{e}$ $Q=1000$

5.14 Fator de casamento na estimação do canal IEEE 802.15.4a (6dB) 119

5.15 Taxa de erro de bits na estimação do canal IEEE 802.15.4a (6dB) 119

5.16 Fator de casamento na estimação do canal IEEE 802.15.4a (8dB) 120

5.17 Taxa de erro de bits na estimação do canal IEEE 802.15.4a (8dB) 120

5.18 Fator de casamento na estimação do canal IEEE 802.15.4a (10dB) 121

5.19 Taxa de erro de bits na estimação do canal IEEE 802.15.4a (10dB) 121

5.20 Fator de casamento na estimação do canal IEEE 802.15.4a (12dB) 122

5.21 Taxa de erro de bits na estimação do canal IEEE 802.15.4a (12dB) 122

5.22 Fator de casamento na estimação do canal IEEE 802.15.4a (14dB) 123

5.23 Taxa de erro de bits na estimação do canal IEEE 802.15.4a (14dB) 123

5.24 Fator de casamento na estimação do canal IEEE 802.15.4a (16dB) 124

5.25 Taxa de erro de bits na estimação do canal IEEE 802.15.4a (16dB) 124

5.26 Fator de casamento na estimação do canal IEEE 802.15.4a (18dB) 125

5.27 Taxa de erro de bits na estimação do canal IEEE 802.15.4a (18dB) 125

5.28 Fator de casamento na estimação do canal IEEE 802.15.4a (20dB) 126

5.29 Taxa de erro de bits na estimação do canal IEEE 802.15.4a (20dB) 126

5.30 BER versus SINR

5.31 Custo computacional relativo

A.1 Diagrama de um Sistema de Comunicações com Espalhamento Direto por Código e Time-Hopping

138

A.2 Diagrama equivalente do Sistema de Comunicações representado na Fig. A.1 


\section{Introdução}

\section{1}

\section{Contextualização deste trabalho}

Um sistema linear e invariante no tempo é considerado esparso quando o vetor que representa sua resposta ao impulso em tempo discreto possui elementos nulos ou desprezíveis. Uma técnica amplamente empregada para estimação de sistemas lineares e invariantes no tempo é a estimação por mínimos quadrados, universalmente conhecida por sua denominação em inglês - Least Squares (LS) Estimation(1). Embora a estimação LS seja uma técnica estatisticamente consistente, sua velocidade de convergência pode ser proibitivamente lenta em algumas aplicações que envolvam a estimação de sistemas esparsos $(2,3)$. Recentes pesquisas relacionadas à Teoria de Compressive Sensing (4, 5) tem motivado o desenvolvimento de técnicas específicas para a estimação (ou identificação) de sistemas esparsos, nos quais a esparsidade do sistema estimado é explorada com o propósito de acelerar a velocidade de convergência do seu processo de estimação.

Em (6), é apresentado o algoritmo Matching Pursuit (MP), que consiste em construir, de forma iterativa, uma estimativa esparsa como uma combinação linear de vetores pertencentes a um conjunto sobredeterminado denominado dicionário. Em (7), uma variação do algoritmo MP é apresentado para fins de estimação de canais esparsos, em que alguns elementos da estimativa LS, com os menores módulos, são zerados e, subsequentemente, uma estimativa esparsa é obtida a partir da reestimação LS dos elementos não-nulos remanescentes. Em (8), é apresentado um algoritmo baseado em MP que constrói a estimativa esparsa através da técnica de gradiente conjugado.

Em (9), é proposto um método de identificação de sistema no contexto de estimação de canal para fins de equalização adaptativa, em que apenas um subconjunto dos elementos do vetor que representa o canal são selecionados e atualizados de forma adaptativa. Em (10), são apresentados alguns algoritmos que tentam detectar elementos nulos no vetor que representa a estimativa esparsa. Um desses algoritmos zera os elementos da estimativa LS cujos módulos estejam abaixo de um limiar pré-estabelecido e, subsequentemente, reconstrói a estimativa LS restrita aos elementos não-nulos remanescentes. Esse processo é repetido iterativamente até que não seja observada nenhuma mudança significativa entre duas estimativas sucessivas. Em (11), é apresentado um algoritmo de hard-thresholding, em que alguns elementos da estimativa 
esparsa são zerados iterativamente até que algum critério de parada seja atendido.

Em (12), o operador LASSO (Least Absolute Shrinkage and Selection Operator) é apresentado, no qual a estimação LS ordinária da resposta ao impulso em tempo discreto do sistema é executada tendo como restrição sua norma $l_{1}$ ser menor que um valor arbitrado. Em (13), é apresentado o critério de otimização denominado Basis Pursuit (BP), o qual estabelece que, em um sistema livre de ruídos, uma representação esparsa da resposta ao impulso do sistema pode ser obtida através da minimização de sua norma $l_{1}$, sujeita à restrição de que o erro de observação seja zero. Em um sistema com ruído aditivo, o critério de otimização do BP é diferente, e equivale à minimização do custo LS acrescido de uma função de regularização que corresponde à norma $l_{1}$ da estimativa multiplicada por uma constante real positiva arbitrária denominada fator de regularização. Em (14), uma família de algoritmos que promovem o encolhimento do suporte da estimativa é proposta.

Em $(15,16,17,18)$, são apresentadas algumas variações do algoritmo Least Mean Squares (LMS), que exploram esparsidade com o objetivo de acelerar a velocidade de convergência na estimação. Em (19), é apresentada uma variação do algoritmo LMS que emprega sequência de Krylov e que tem como vantagens não utilizar nenhuma informação a priori do sistema estimado e não se limitar à estimação de sistemas esparsos.

Em (20), é apresentada uma família de algoritmos Recursive Least Squares (RLS) que incorporam regularização convexa. Dentre eles, é apresentado o algoritmo $l_{1}$-RLS, utilizado no presente trabalho como benchmark na avaliação de desempenho e de esforço computacional dos métodos de estimação de sistemas esparsos originalmente elaborados e apresentados nesta Tese.

Em (21), é apresentado um algoritmo que minimiza o custo LS mais a norma $l_{1}$ empregando uma função homotópica que produz uma sequência decrescente de valores ao parâmetro de regularização. Em (22), é apresentado um algoritmo para estimação de sistemas esparsos que emprega homotopia. Em (23, 24), são apresentados dois trabalhos que abordam o ajuste automático dos parâmetros de regularização.

Em (25), é apresentado um método de estimação baseado no algoritmo Normalized-LMS (NLMS) que emprega filtros paralelos. Em (26), é proposto um método de equalização em sistemas Direct-Squence Ultra-Wideband (DSUWB) que explora a esparsidade do canal e que emprega múltiplos filtros paralelos e técnicas de posto reduzido. 
O enfoque desta Tese está direcionado ao desenvolvimento e à apresentação de métodos originais para estimação de sistemas esparsos, de baixa complexidade computacional, baseados em estimação por mínimos quadrados e que incorporam técnicas relacionadas à otimização convexa e à Teoria de Compressive Sensing.

\section{2}

\section{Motivação e objetivo}

A atual proliferação de Redes de Sensores baseados na tecnologia ImpulseRadio Ultra-Wideband (IR-UWB), de amplo interesse em aplicações civis e militares, foi uma das motivações para a realização deste trabalho, haja vista que os canais nos quais operam os sistemas IR-UWB são tipicamente esparsos. Em relação ao problema específico de estimação LS de canais esparsos em sistemas de comunicações, reside aí a seguinte razão custo-benefício: ou a sequência de treinamento é longa, para que o erro de estimação seja pequeno o suficiente para que a taxa de erros de bits fique abaixo de um valor máximo admitido, ao custo da redução da taxa efetiva de transmissão, ou a sequência de treinamento é curta, aumentando-se assim a taxa efetiva de transmissão, ao custo do aumento da taxa de erros de bits.

O problema de estimação LS de canais esparsos, em particular em sistemas de comunicações baseados na tecnologia IR-UWB, motivou o desenvolvimento de quatro métodos de baixa complexidade computacional projetados especificamente para a estimação de sistemas esparsos. Estes métodos são originalmente elaborados e apresentados no Capítulo 4. São eles: Encolhimentos Sucessivos, Expansões Sucessivas, Minimização da Norma $l_{1}$ e Ajuste automático do fator de regularização do Custo LS. Esses quatro métodos são baseados na técnica de estimação LS e, com o objetivo de acelerar a velocidade de convergência do processo de estimação, incorporam técnicas relacionadas à otimização convexa e à Teoria de Compressive Sensing. A apresentação desses quatro métodos é feita de maneira unificada tendo como elo de ligação o mesmo critério para o relaxamento do custo LS.

O método de Encolhimentos Sucessivos guarda semelhanças com algumas técnicas de hard-tresholding (11) e provoca, de forma controlada, sucessivos encolhimentos do suporte da estimativa da resposta ao impulso do sistema, até que o custo LS exceda um limiar previamente arbitrado. O método de Expansões Sucessivas foi inspirado no algoritmo de Matching Pursuit (6) e, em oposição ao método anterior, provoca, de maneira controlada, sucessivas expansões do suporte da estimativa da resposta ao impulso do sistema, até 
que o custo LS fique abaixo de um limiar previamente arbitrado. O método de Minimização da Norma $l_{1}$ foi inspirado nas técnicas de Basis Pursuit (13) e no operador LASSO (Least Absolute Shrinkage and Selection Operator) (12), e guarda muita semelhança com o método de Encolhimentos Sucessivos. Porém, a diferença fundamental entre esses dois métodos é a seguinte: enquanto o método de Encolhimentos Sucessivos baseia-se na consistência estatística da técnica de estimação LS com suporte reduzido, o método de minimização da norma $l_{1}$ baseia-se em argumentos geométricos, mais precisamente nas condições de contorno associadas ao problema de otimização convexa intrínseco no método. O método de Ajuste Automático do Fator de Regularização do custo LS foi inspirado nas técnicas baseadas na regularização da funçãocusto $(17,20)$ e tem, como principal mérito, a busca automática pelo fator de regularização ótimo. Sucedendo a apresentação de cada um dos métodos, são apresentados resultados de simulações em que seus desempenhos são comparados com as técnicas de estimação LS ordinária e LS Oráculo (que corresponde à estimação LS com suporte reduzido em que o suporte da resposta ao impulso do canal é conhecido a priori) e a análise de custo computacional.

\section{3}

\section{Organização do texto}

No Capítulo 2, é feita uma breve apresentação do método ordinário de estimação por mínimos quadrados de sistemas lineares e invariantes no tempo, universalmente conhecido por sua denominação em inglês - Least Squares (LS) Estimation. Neste capítulo, é também apresentada uma forma de realizar a estimação LS através de processamento em blocos do sinal observado na saída do sistema a ser estimado.

No Capítulo 3, é apresentada, com uma abordagem inédita, a técnica de estimação LS com suporte reduzido, cujo arcabouço teórico fundamenta os quatro métodos de estimação de sistemas esparsos originalmente apresentados nesta Tese. Neste capítulo, também são realizadas a análise de convergência e do custo computacional da estimação LS com suporte reduzido.

No Capítulo 4, o problema de estimação LS ótima de sistemas esparsos é caracterizado. Em seguida, é estabelecida, de maneira inédita, uma conexão entre a norma $l_{1}$ (amplamente empregada de forma heurística como medida de esparsidade) e a pseudo-norma $l_{0}$. Finalmente, os quatro métodos de estimação de sistemas esparsos, originalmente desenvolvidos ao longo desta Tese, são apresentados. São eles: Encolhimentos Sucessivos, Expansões Sucessivas, Minimização da Norma $l_{1}$ e Ajuste automático do fator de regularização do Custo 
LS. Esses quatro métodos são abordados de forma unificada, tendo como elo de ligação um critério único para o relaxamento do custo LS.

O Capítulo 5 é dedicado à avaliação de desempenho e de custo computacional dos métodos propostos, a partir de resultados de simulações em computador, comparando-os entre si e com a técnica $l_{1}$-RLS (20). Os resultados das simulações são organizados em duas partes: na primeira, as simulações são dedicadas à estimação de Sistemas Esparsos de Máxima Entropia (o conceito de Sistemas Esparsos de Máxima Entropia é introduzido, de forma inédita, no Anexo B); na segunda, as simulações são dedicadas ao caso de particular interesse de avaliar a taxa de erro de bits em sistemas UWB que envolvem a estimação de canais esparsos, baseados no modelo de canal IEEE 802.15.4a (27) (o modelo adotado para representação de sinais em sistemas UWB é apresentado no Anexo A). Os resultados das simulações demonstram que os métodos originalmente apresentados nesta Tese têm desempenho acentuadamente superior ao apresentado pelo algoritmo $l_{1}$-RLS, em muitos casos alcançando o mesmo desempenho ótimo do algoritmo de benchmark LS Oráculo.

O Capítulo 6 fecha este trabalho com a apresentação de uma análise sintética dos métodos propostos e aponta oportunidades de continuidade nas pesquisas.

\section{4}

\section{Notação adotada}

Caracteres maiúsculos em negrito denotam matrizes (por exemplo, X). Caracteres minúsculos em negrito denotam vetores (por exemplo, $\mathbf{x}$ ). Caracteres maiúsculos em negrito vazado denotam conjuntos (por exemplo, $\mathbb{X}$ ).

Os símbolos $\star^{T H} \dagger$ indicam, respectivamente, os seguintes operadores: convolução linear, transposição, hermitiano e pseudo-inversa. O símbolo $\leftarrow$ denota atribuição. O símbolo $\stackrel{a . s}{\longrightarrow}$ denota convergência com probabilidade 1 (almost sure convergence).

O operador $|\mathbb{X}|$ denota a cardinalidade de $\mathbb{X}$. O operador $\Re(x)$ denota a parte real de $x$. O operador $\lceil x\rceil$ denota o menor inteiro maior ou igual a $x$. 


\section{2}

\section{Estimação LS de sistemas lineares e invariantes no tempo}

\section{1}

\section{Introdução}

Nesta Tese, quatro novos métodos de estimação de sistemas esparsos são originalmente desenvolvidos e apresentados. São eles: Encolhimentos Sucessivos, Expansões Sucessivas, Minimização da Norma $l_{1}$ e Regularização Automática do Custo LS.

Estes quatro métodos baseiam-se na técnica de estimação de sistemas lineares e invariantes no tempo por mínimos quadrados, universalmente conhecida pela sua denominação em inglês - Least Squares (LS) Estimation. Por isso, neste capítulo, a técnica de estimação LS é sumariamente apresentada, bem como o modelo adotado ao longo da Tese para representar o esquema de estimação de sistemas (esparsos) lineares e invariantes no tempo.

A técnica de estimação LS é uma das mais comumente empregadas para a estimação (ou identificação) de sistemas lineares e invariantes no tempo. No caso da estimação de sistemas lineares e invariantes no tempo representados em tempo discreto, a estimação LS fornece como resultado a estimativa da resposta ao impulso em tempo discreto do sistema que minimiza a média aritmética das normas ao quadrado dos erros de observação, que correspondem às diferenças entre os vetores de observação (isto é, os vetores que representam o sinal na saída do sistema) e os vetores que representam o sinal de saída reconstituído (isto é, o sinal resultante da convolução entre o sinal de entrada e a estimativa das resposta ao impulso discreta do sistema). Esta estimativa, denominada estimativa LS, é única (28).

Quando os erros de observação constituem-se em um processo estocástico gaussiano branco, é possível demonstrar que as estimativas LS são ótimas, no sentido de que correspondem às estimativas que atendem ao critério de máxima verossimilhança. Mesmo quando a caracterização estatística dos erros de observação é desconhecida, a estimação LS costuma apresentar bom desempenho. Além disso, a estimação LS é uma técnica amplamente empregada mesmo quando a caracterização estatística do erro de observação é conhecida e não corresponde a um processo estocástico gaussiano branco. Isto se deve ao fato de que a estimação LS proporciona um tratamento matemático simples para a estimação de sistemas lineares e invariantes no tempo. 
A estimação LS produz estimativas LS consistentes: isto é, quanto maior a quantidade de vetores de observação processados na geração da estimativa, menor a variância do erro de estimação, que corresponde à norma da diferença entre os vetores que representam a estimativa LS e a verdadeira resposta ao impulso em tempo discreto do sistema. A consistência e a baixa complexidade da estimação LS foram as razões para restringir as pesquisas e o desenvolvimento de novos métodos de estimação de sistemas esparsos a essa modalidade de estimação. Técnicas de caráter estocástico, tal qual o algoritmo LMS, não foram objetos de pesquisa nesta Tese, por apresentarem desvantagens em relação à técnica de estimação LS, tais como baixa velocidade de convergência e sempre apresentar um patamar de erro de estimação (1).

Uma outra qualidade da estimação LS é que ela pode ser modificada para produzir adaptativamente estimativas de sistemas variantes no tempo, como por exemplo a técnica Recursive Least Squares (RLS), que tem como parâmetro um fator de esquecimento (1). Embora a estimação de sistemas variantes no tempo esteja fora do escopo desta Tese, as técnicas aqui apresentadas também podem ser adaptadas para esta finalidade.

Apesar de todas as qualidades da estimação LS, quando aplicada à estimação de sistemas esparsos, se a quantidade de vetores de observação processados não for muito grande, o erro de estimação pode não ser pequeno o suficiente, dependendo da aplicação. Por exemplo, em sistemas de comunicações, a estimação LS de canais esparsos depara-se com a seguinte relação custobenefício: ou o tamanho da sequência de treinamento é longa a fim de que o erro de estimação seja pequeno o suficiente para que a probabilidade de erro de bits fique abaixo do limite tolerável pelo sistema, às custas da redução da taxa efetiva de transmissão, ou o tamanho da sequência de treinamento é curta, aumentando-se assim a taxa efetiva de transmissão, às custas do aumento da taxa de erro de bits do sistema. Por isso, nos capítulos seguintes a técnica de estimação LS será adaptada para que a velocidade de convergência da estimação LS de sistemas esparsos seja acelerada, destacandose o desenvolvimento da técnica de estimação LS com suporte reduzido. Além disso, com o objetivo de reduzir seu custo computacional, nesse trabalho é apresentada a técnica de processamento em blocos da estimação LS.

\section{2}

\section{Esquema de Estimação LS}

Seja $\mathbf{h}$ o vetor-coluna de comprimento $L$ que representa a resposta ao impulso em tempo discreto de um sistema linear e invariante no tempo $\mathcal{H}$. Seja $\overline{\mathbf{c}}$ o 
vetor-coluna de comprimento $N$ que representa, em tempo discreto, um sinal na entrada de $\mathcal{H}$. O sinal em tempo discreto na saída de $\mathcal{H}$ pode ser representado pelo vetor-coluna $\overline{\mathbf{s}}$, de comprimento $P=L+N-1$, dado por

$$
\overline{\mathbf{s}}=\overline{\mathbf{c}} \star \mathbf{h}=\overline{\mathbf{C}} \mathbf{h}
$$

em que * representa a operação discreta de convolução e $\overline{\mathbf{C}}$ é matriz Toeplitz, de dimensão $P \times L$, cujo produto $\overline{\mathbf{C}} \mathbf{h}$ representa a convolução entre $\overline{\mathbf{c}}$ e $\mathbf{h}$.

Seja $\overline{\mathbf{r}}$ o vetor de observação, dado por

$$
\overline{\mathbf{r}}=\overline{\mathbf{s}}+\overline{\mathbf{z}}=\overline{\mathbf{C}} \mathbf{h}+\overline{\mathbf{z}}
$$

em que $\overline{\mathbf{z}}$ representa o erro de observação, de natureza estocástica.

A estimativa LS da resposta ao impulso em tempo discreto de $\mathcal{H}$ é definida como

$$
\hat{\mathbf{h}} \triangleq \arg \min _{\mathbf{x}}\|\overline{\mathbf{r}}-\overline{\mathbf{C}} \mathbf{x}\|^{2}
$$

O problema de minimização em 2-3 é convexo e, portanto, possui um único mínimo global. Calculando o gradiente de 2-3 e igualando a zero, tem-se que

$$
\hat{\mathbf{h}}=\overline{\mathbf{C}}^{\dagger} \overline{\mathbf{r}}
$$

em que $\overline{\mathbf{C}}^{\dagger} \triangleq\left(\overline{\mathbf{C}}^{H} \overline{\mathbf{C}}\right)^{-1} \overline{\mathbf{C}}^{H}$ é a matriz pseudo-inversa de $\overline{\mathbf{C}}$.

Alternativamente, a estimativa LS pode ser calculada também da seguinte forma

$$
\hat{\mathbf{h}}=\overline{\mathbf{A}}^{-1} \overline{\mathbf{b}}
$$

em que $\overline{\mathbf{A}}=\overline{\mathbf{C}}^{H} \overline{\mathbf{C}}$ e $\overline{\mathbf{b}}=\overline{\mathbf{C}}^{H} \overline{\mathbf{r}}$.

Desde que $\overline{\mathbf{A}}$ seja de posto cheio, ela também é positiva-definida e, nesse caso, é possível calcular $\hat{\mathbf{h}}$ consumindo apenas $2 / 3$ das operações de ponto flutuante em relação a 2-5 (29), da seguinte forma

$$
\hat{\mathbf{h}}=\overline{\mathbf{L}}^{H} \backslash_{T}\left(\overline{\mathbf{L}} \backslash_{T} \overline{\mathbf{b}}\right)
$$

em que $\overline{\mathbf{L}}$ é a matriz triangular inferior obtida através da Decomposição de Cholesky de $\overline{\mathbf{A}}$ (ou seja, $\overline{\mathbf{A}}=\overline{\mathbf{L}}^{H} \overline{\mathbf{L}}$ ) e $\backslash_{T}$ representa a operação de divisão à esquerda por matriz triangular. 


\section{3}

\section{Processamento em blocos da estimação LS}

O cálculo de $\hat{\mathbf{h}}$ a partir de 2-3 exige o armazenamento em memória de todo o vetor de observação $\overline{\mathbf{r}}$, o que pode ser uma dificuldade, se o seu comprimento $P$ for muito grande em relação à capacidade de armazenamento do dispositivo que executa a estimação LS do sistema. Uma alternativa é o processamento da estimação LS em blocos, que consiste em quebrar o vetor de observação em blocos $\mathbf{r}_{i}$ de comprimento $M \leq P$.

Seja $\mathbb{L}=\{1,2, \ldots, L\}$ e $\mathbb{P}_{i}$ o conjunto dos índices das linhas correspondentes ao $i$-ésimo bloco, dado por

$$
\mathbb{P}_{i}=\{(i-1) M+1, \ldots, i M\}
$$

Suponha, sem perda de generalidade, que $P$ seja múltiplo de $M$ tal que $P=N_{b} M$, em que $N_{b}$ é a quantidade de blocos (se $P$ não for múltiplo de $M$, o vetor $\overline{\mathbf{c}}$ que representa o sinal na entrada de $\mathcal{H}$ pode ser acrescido de zeros até que o vetor de observação resultante $\overline{\mathbf{r}}$ tenha comprimento múltiplo de $M)$. Nesse caso, a equação 2-3 pode ser reescrita da seguinte forma

$$
\hat{\mathbf{h}} \triangleq \arg \min _{\mathbf{x}} \sum_{i=1}^{N_{b}}\left\|\mathbf{r}_{i}-\mathbf{C}_{i} \mathbf{x}\right\|^{2}
$$

em que $\mathbf{r}_{i}$ é o vetor-coluna, de comprimento $M$, correspondente ao $i$-ésimo bloco de observação, dado por

$$
\mathbf{r}_{i}=(\overline{\mathbf{r}})_{\mathbb{P}_{i}, \mathbb{L}}
$$

e $\mathbf{C}_{i}$ corresponde ao $i$-ésimo sub-bloco da matriz $\overline{\mathbf{C}}$, dado por

$$
\mathbf{C}_{i}=(\overline{\mathbf{C}})_{\mathbb{P}_{i}, \mathbb{L}}
$$

Seja $\hat{\mathbf{h}}_{k}$ a estimativa LS no instante $k$, definida como

$$
\hat{\mathbf{h}}_{k} \triangleq \arg \min _{\mathbf{x}} J_{k}(\mathbf{x}) \quad 1 \leq k \leq N_{b}
$$

em que $J_{k}(\mathbf{x}): \mathbb{C}^{L} \rightarrow \mathbb{R}^{+}$é o custo LS no instante $k$, definido como

$$
J_{k}(\mathbf{x}) \triangleq \frac{1}{k} \sum_{i=1}^{k}\left\|\mathbf{r}_{i}-\mathbf{C}_{i} \mathbf{x}\right\|^{2}
$$

Comparando as equações $2-8$ e 2-11, verifica-se que $\hat{\mathbf{h}}=\hat{\mathbf{h}}_{k}$ quando $k=N_{b}$.

A função-custo $J_{k}(\mathbf{x})$ em 2-12 pode ser reescrita como 


$$
J_{k}(\mathbf{x})=R_{k}-2 \Re\left[\mathbf{b}_{k}^{H} \mathbf{x}\right]+\mathbf{x}^{H} \mathbf{A}_{k} \mathbf{x}
$$

em que

$$
\begin{aligned}
R_{k} & \triangleq \frac{1}{k} \sum_{i=1}^{k}\left\|\mathbf{r}_{i}\right\|^{2} \\
\mathbf{A}_{k} & \triangleq \frac{1}{k} \sum_{i=1}^{k} \mathbf{C}_{i}^{H} \mathbf{C}_{i} \\
\mathbf{b}_{k} & \triangleq \frac{1}{k} \sum_{i=1}^{k} \mathbf{C}_{i}^{H} \mathbf{r}_{i}
\end{aligned}
$$

Igualando o gradiente de $J_{k}(\mathbf{x})$ a zero, verifica-se que a estimativa LS $\hat{\mathbf{h}}_{k}$, dada por $2-11$, pode ser calculada da seguinte forma

$$
\hat{\mathbf{h}}_{k}=\mathbf{A}_{k}^{-1} \mathbf{b}_{k}
$$

É importante observar que uma condição necessária para que $\mathbf{A}_{k}$ possua posto completo $L$ é que

$$
k M \geq L
$$

Portanto, para que 2-15 possua solução determinada, a condição em 2-16 deve ser satisfeita.

Observando que $\mathbf{A}_{k}$ é matriz positiva-definida, é possível calcular $\hat{\mathbf{h}}_{k}$ consumindo apenas 2/3 das operações de ponto flutuante em relação a 2-15 (29), da seguinte forma

$$
\hat{\mathbf{h}}_{k}=\mathbf{L}_{k}^{H} \backslash_{T}\left(\mathbf{L}_{k} \backslash_{T} \mathbf{b}_{k}\right)
$$

em que $\mathbf{L}_{k}$ é a matriz triangular inferior obtida através da Decomposição de Cholesky de $\mathbf{A}_{k}$ (ou seja, $\mathbf{A}_{k}=\mathbf{L}_{k}^{H} \mathbf{L}_{k}$ ).

\section{4}

\section{Generalização do processamento em blocos da estimação LS}

Observando as equações 2-2, 2-9 e 2-10, verifica-se que cada bloco $\mathbf{r}_{i}$ pode ser representado da seguinte forma

$$
\mathbf{r}_{i}=\mathbf{C}_{i} \mathbf{h}+\mathbf{z}_{i}
$$

em que $\mathbf{z}_{i}$ é o vetor-coluna, de comprimento $M$, correspondente ao $i$-ésimo bloco de erros de observação, dado por

$$
\mathbf{z}_{i}=(\overline{\mathbf{z}})_{\mathbb{P}_{i}, \mathbb{L}}
$$


A equação 2-18 pode ser generalizada para representar não somente o $i$-ésimo bloco de $\overline{\mathbf{r}}$, mas para representar também o $i$-ésimo vetor de observação $\mathbf{r}_{i}$ em um esquema de estimação em que, em vez do emprego de um único sinal $\overline{\mathbf{c}}$ na entrada do sistema a ser estimado $\mathcal{H}$, é empregada uma sequência de sinais de entrada independentes entre si $\left\{\mathbf{c}_{i}, i=1,2, \ldots\right\}$.

Uma aplicação de particular interesse é a estimação de canais em sistemas de comunicações com esquema de múltiplo acesso baseado em espalhamento direto, tais quais alguns padrões CDMA (Code Division Multiple Access) e UWB (Ultra-wideband). Nesse caso, $\mathbf{c}_{i}$ passa a representar o $i$-ésimo código de espalhamento direto transmitido através do canal $\mathcal{H}$, cuja resposta ao impulso em tempo discreto é $\mathbf{h}$. Assim, $\mathbf{C}_{i}$ é matriz Toeplitz cujo produto $\mathbf{C}_{i} \mathbf{h}$ representa a convolução entre $\mathbf{c}_{i}$ e $\mathbf{h}$. O cálculo de $\hat{\mathbf{h}}_{k}$, que representa a estimativa LS da resposta ao impulso de $\mathcal{H}$ no instante $k$, permanece exatamente igual como foi apresentado na seção anterior.

O Anexo A apresenta a construção do modelo equivalente discreto em banda básica para o esquema de transmissão e recepção de alguns padrões CDMA e do padrão UWB IEEE 802.15.4a.

\section{5}

\section{Considerações finais}

Neste capítulo, foi realizada uma breve recapitulação sobre a técnica de estimação LS. Em seguida, foi apresentada uma forma de realizar a estimação LS a partir da fragmentação do vetor de observação em blocos.

A estimação LS apresentada neste capítulo é denominada ao longo desta Tese como Estimação LS Full-support (de suporte completo). No capítulo a seguir, será apresentada a técnica de estimação LS com suporte reduzido, na qual é suposto a priori que um ou mais elementos da estimativa $\hat{\mathbf{h}}_{k}$ sejam iguais a zero. A estimação LS Full-support consiste em um caso particular da estimação LS com suporte reduzido. A estimação LS com suporte reduzido constitui-se na base de três das quatro técnicas de estimação de sistemas esparsos apresentados originalmente apresentadas nesta Tese (Encolhimentos Sucessivos, Expansões Sucessivas e Minimização da Norma $l_{1}$ ). 


\section{3}

\section{Estimação Least Squares com suporte reduzido}

\section{1}

\section{Introdução}

No capítulo anterior, a técnica de estimação LS full-support ordinária foi sumariamente apresentada. Em seguida, foi apresentada uma forma de executar a estimação LS full-support através do processamento em blocos (fragmentos) do vetor de observação. Apesar das virtudes da estimação LS, dentre as quais destacam-se a produção de estimativas consistentes e despolarizadas, sua convergência pode ser lenta quando aplicada à estimação de um sistema linear e invariante no tempo esparso $(2,3)$, cuja resposta ao impulso em tempo discreto possa ser representada por um vetor-coluna de comprimento longo, mas apenas uma quantidade pequena de seus elementos significativos, com os elementos restantes nulos ou desprezíveis.

Uma forma de acelerar a estimação LS de sistemas esparsos (como por exemplo, a estimação de canais esparsos em sistemas de comunicações), reduzindo-se assim as diferenças entre a estimativa LS e a resposta ao impulso do sistema quando a quantidade de observações processadas é pequena, é restringir adequadamente o suporte da estimação. Dessa forma, a estimação LS de sistemas esparsos pode ser vista como um problema de se determinar qual o suporte mais adequado para representar a resposta ao impulso do sistema, considerando-se o conjunto de vetores de observação processados. Consequentemente, a estimação LS de sistemas esparsos envolve o estabelecimento de algum critério para determinar quais os elementos da estimativa LS full-support ordinária podem ser, de fato, considerados desprezíveis e, portanto, descartáveis.

Neste capítulo, é apresentada uma adaptação da técnica de estimação LS fullsupport ordinária para um modelo de estimação LS com suporte reduzido. Nesse aspecto, a estimação LS full-support pode ser vista como um caso particular da estimação LS com suporte reduzido. Em seguida, é realizada uma análise de convergência do custo LS e do erro de estimação produzidos pela técnica de estimação LS com suporte reduzido. As conclusões apresentadas neste capítulo são usadas para fundamentar os seguintes métodos de estimação de sistemas esparsos originalmente elaborados e apresentados no capítulo seguinte: Encolhimentos Sucessivos, Expansões Sucessivas e Minimização da Norma $l_{1}$. 


\section{2}

\section{Apresentação do esquema de estimação LS com suporte reduzido}

Considere o problema de estimação da resposta ao impulso discreta de um sistema linear e invariante no tempo $\mathcal{H}$ em que o sinal observado na saída do sistema, no instante $k$, pode ser representado pelo vetor de observação $\mathbf{r}_{k}$, dado pela equação 2-18, repetida aqui por conveniência

$$
\mathbf{r}_{k}=\mathbf{C}_{k} \mathbf{h}+\mathbf{z}_{k}
$$

em que:

- $\mathbf{r}_{k}$ é um vetor complexo de dimensão $M \times 1$, com $M=N+L-1$;

- h é um vetor complexo de dimensão $L \times 1$ que representa a resposta ao impulso discreta de $\mathcal{H}$;

- $\mathbf{C}_{k}$ é uma matriz Toeplitz de dimensão $M \times L$ cujo produto $\mathbf{C}_{k} \mathbf{h}$ representa a convolução entre uma sequência de $N$ símbolos $\mathbf{c}_{k}$ na entrada de $\mathcal{H}\left(\operatorname{com} E\left\{\left\|\mathbf{c}_{k}\right\|^{2}\right\}=\mathcal{E}_{c}<\infty\right.$ para todo $k$ ) e a resposta ao impulso discreta $\mathbf{h}$;

- $\mathbf{z}_{k}$ é um vetor complexo de dimensão $M \times 1$ que representa o erro de observação na saída de $\mathcal{H}$.

Seja $\mathbb{S}_{\mathbf{h}}$ o suporte (conjunto dos índices dos elementos não nulos) de $\mathbf{h}$, isto é,

$$
\mathbb{S}_{\mathbf{h}}=\left\{i \in \mathbb{L} \mid \mathbf{h}_{i} \neq 0\right\}
$$

em que $\mathbb{L}=\{1,2, \ldots, L\}$. Seja $\mathbb{S}$ um superconjunto qualquer de $\mathbb{S}_{\mathbf{h}}$ (isto é, $\mathbb{L} \supseteq \mathbb{S} \supseteq \mathbb{S}_{\mathbf{h}}$ ); nesse caso, 3-1 pode ser reescrita da seguinte forma

$$
\mathbf{r}_{k}=\left(\mathbf{C}_{k}\right)_{\mathbb{S}}(\mathbf{h})_{\mathbb{S}}+\mathbf{z}_{k}
$$

em que $\left(\mathbf{C}_{k}\right)_{\mathbb{S}}$ é uma matriz de dimensão $M \times|\mathbb{S}|$ formada pelas colunas de $\mathbf{C}_{k}$ cujos índices pertencem a $\mathbb{S}$ e $(\mathbf{h})_{\mathbb{S}}$ é um vetor de dimensão $|\mathbb{S}| \times 1$ formado pelos elementos de $\mathbf{h}$ cujos índices pertencem a $\mathbb{S}$ (aqui, |.| denota a cardinalidade do conjunto).

Seja $\hat{\mathbf{h}}_{k}^{\mathbb{S}}$ o vetor de dimensão $|\mathbb{S}| \times 1$ que representa a estimativa da resposta ao impulso discreta do sistema, no instante $k$, associada a um suporte genérico $\mathbb{S} \subseteq \mathbb{L}$, dado por

$$
\hat{\mathbf{h}}_{k}^{\mathbb{S}}=\arg \min _{\mathbf{x}} J_{k}^{\mathbb{S}}(\mathbf{x})
$$


em que $J_{k}^{\mathbb{S}}(\mathbf{x}): \mathbb{C}^{|\mathbb{S}|} \rightarrow \mathbb{R}^{+}$é a função-custo associada ao suporte $\mathbb{S}$ que fornece a média aritmética dos erros quadráticos, definida como

$$
J_{k}^{\mathbb{S}}(\mathbf{x}) \triangleq \frac{1}{k} \sum_{i=1}^{k}\left\|\mathbf{r}_{i}-\left(\mathbf{C}_{i}\right)_{\mathbb{S}} \mathbf{x}\right\|^{2}
$$

A função-custo $J_{k}^{\mathbb{S}}(\mathbf{x})$ pode ser reescrita da seguinte forma

$$
J_{k}^{\mathbb{S}}(\mathbf{x})=R_{k}-2 \Re\left[\left(\mathbf{b}_{k}\right)_{\mathbb{S}}^{H} \mathbf{x}\right]+\mathbf{x}^{H}\left(\mathbf{A}_{k}\right)_{\mathbb{S}, \mathbb{S}} \mathbf{x}
$$

em que $R_{k}, \mathbf{A}_{k}$ e $\mathbf{b}_{k}$ são definidos na equação 2-14, repetida aqui por conveniência

$$
\begin{aligned}
R_{k} & \triangleq \frac{1}{k} \sum_{i=1}^{k}\left\|\mathbf{r}_{i}\right\|^{2} \\
\mathbf{A}_{k} & \triangleq \frac{1}{k} \sum_{i=1}^{k} \mathbf{C}_{i}^{H} \mathbf{C}_{i} \\
\mathbf{b}_{k} & \triangleq \frac{1}{k} \sum_{i=1}^{k} \mathbf{C}_{i}^{H} \mathbf{r}_{i}
\end{aligned}
$$

e $\left(\mathbf{b}_{k}\right)_{\mathbb{S}}$ é o vetor de dimensão $|\mathbb{S}| \times 1$ formado pelos elementos de $\mathbf{b}_{k}$ cujos índices pertencem a $\mathbb{S}$ e $\left(\mathbf{A}_{k}\right)_{\mathbb{S}, \mathbb{S}}$ é a matriz de dimensão $|\mathbb{S}| \times|\mathbb{S}|$ formada pelos elementos de $\mathbf{A}_{k}$ cujos índices de suas linhas e de suas colunas pertencem a $\mathbb{S}$. A função-custo $J_{k}^{\mathbb{S}}(\mathbf{x})$ é quadrática em $\mathbf{x}$ e, portanto, convexa. Além disso, o domíniio de $J_{k}^{\mathbb{S}}($.$) é conjunto fechado. Logo, J_{k}^{\mathbb{S}}(\mathbf{x})$ possui um único valor mínimo global. O gradiente de $J_{k}^{\mathbb{S}}(\mathbf{x})$ é dado por

$$
\nabla J_{k}^{\mathbb{S}}(\mathbf{x})=2\left[\left(\mathbf{A}_{k}\right)_{\mathbb{S}, \mathbb{S}} \mathbf{x}-\left(\mathbf{b}_{k}\right)_{\mathbb{S}}\right]
$$

A solução de 3-4 é obtida resolvendo-se $\nabla J_{k}^{\mathbb{S}}\left(\hat{\mathbf{h}}_{k}^{\mathbb{S}}\right)=\mathbf{0}$, que é dada por

$$
\hat{\mathbf{h}}_{k}^{\mathbb{S}}=\left(\mathbf{A}_{k}\right)_{\mathbb{S}, \mathbb{S}}^{-1}\left(\mathbf{b}_{k}\right)_{\mathbb{S}}
$$

Seja $J_{k}(\mathbb{S})$ o mínimo erro médio quadrático associado ao suporte $\mathbb{S}$, ou seja,

$$
J_{k}(\mathbb{S})=J_{k}^{\mathbb{S}}\left(\hat{\mathbf{h}}_{k}^{\mathbb{S}}\right)
$$

Aplicando 3-9 em 3-6 e observando que $\left(\mathbf{A}_{k}\right)_{\mathbb{S}, \mathbb{S}}^{-1}$ é matriz positiva-definida, verifica-se que $J_{k}(\mathbb{S})$ é dado por

$$
J_{k}(\mathbb{S})=R_{k}-\left(\mathbf{b}_{k}\right)_{\mathbb{S}}^{H}\left(\mathbf{A}_{k}\right)_{\mathbb{S}, \mathbb{S}}^{-1}\left(\mathbf{b}_{k}\right)_{\mathbb{S}}
$$

ou equivalentemente, substituindo 3-9 em 3-11, verifica-se que

$$
J_{k}(\mathbb{S})=R_{k}-\left(\mathbf{b}_{k}\right)_{\mathbb{S}}^{H} \hat{\mathbf{h}}_{k}^{\mathbb{S}}
$$

Como $\left(\mathbf{A}_{k}\right)_{\mathbb{S}, \mathbb{S}}$ é matriz positiva-definida, ela pode ser expressa como 


$$
\left(\mathbf{A}_{k}\right)_{\mathbb{S}, \mathbb{S}}=\left(\mathbf{L}_{k}^{\mathbb{S}}\right)^{H} \mathbf{L}_{k}^{\mathbb{S}}
$$

em que $\mathbf{L}_{k}^{\mathbb{S}}$ é matriz triangular inferior, resultante da Decomposição de Cholesky da matriz $\left(\mathbf{A}_{k}\right)_{\mathbb{S}, \mathbb{S}}$. Aplicando-se 3-13, 3-11 pode ser reescrita como

$$
J_{k}(\mathbb{S})=R_{k}-\left\|\left(\mathbf{L}_{k}^{\mathbb{S}}\right)^{-1}\left(\mathbf{b}_{k}\right)_{\mathbb{S}}\right\|^{2}
$$

A equação 3-14 é uma forma de se calcular $J_{k}(\mathbb{S})$ sem a necessidade de conhecer $\hat{\mathbf{h}}_{k}^{\mathbb{S}}$ e será útil em alguns algoritmos propostos no próximo capítulo.

\section{3}

\section{Composição da estimativa LS com suporte reduzido}

Combinando 3-1, 3-7 e 3-9, a estimativa esparsa do sistema, associada ao suporte $\mathbb{S}$, no instante $k$, pode ser reescrita como

$$
\hat{\mathbf{h}}_{k}^{\mathbb{S}}=\left(\mathbf{A}_{k}\right)_{\mathbb{S}, \mathbb{S}}^{-1}\left[\frac{1}{k} \sum_{i=1}^{k}\left(\mathbf{C}_{i}\right)_{\mathbb{S}}^{H}\left(\mathbf{C}_{i} \mathbf{h}+\mathbf{z}_{i}\right)\right]
$$

Observando que $\mathbf{C}_{i} \mathbf{h}=\left(\mathbf{C}_{i}\right)_{\mathbb{S}}(\mathbf{h})_{\mathbb{S}}+\left(\mathbf{C}_{i}\right)_{\overline{\mathbb{S}}}(\mathbf{h})_{\overline{\mathbb{S}}}$, em que $\overline{\mathbb{S}}=\mathbb{L} \backslash \mathbb{S}$ é o complemento de $\mathbb{S}$ (isto é, $\mathbb{S} \cup \overline{\mathbb{S}}=\mathbb{L}$ e $\mathbb{S} \cap \overline{\mathbb{S}}=\emptyset$ ), então 3 -15 pode ser reescrita como

$$
\hat{\mathbf{h}}_{k}^{\mathbb{S}}=\left(\mathbf{A}_{k}\right)_{\mathbb{S}, \mathbb{S}}^{-1}\left\{\frac{1}{k} \sum_{i=1}^{k}\left(\mathbf{C}_{i}\right)_{\mathbb{S}}^{H}\left\{\left[\left(\mathbf{C}_{i}\right)_{\mathbb{S}}(\mathbf{h})_{\mathbb{S}}+\left(\mathbf{C}_{i}\right)_{\overline{\mathbb{S}}}(\mathbf{h})_{\overline{\mathbb{S}}}\right]+\mathbf{z}_{i}\right\}\right\}
$$

Verifica-se que $\frac{1}{k} \sum_{i=1}^{k}\left(\mathbf{C}_{i}\right)_{\mathbb{S}}^{H}\left(\mathbf{C}_{i}\right)_{\mathbb{S}}=\left(\mathbf{A}_{k}\right)_{\mathbb{S}, \mathbb{S}}$ e $\frac{1}{k} \sum_{i=1}^{k}\left(\mathbf{C}_{i}\right)_{\mathbb{S}}^{H}\left(\mathbf{C}_{i}\right)_{\overline{\mathbb{S}}}=\left(\mathbf{A}_{k}\right)_{\mathbb{S}, \overline{\mathbb{S}}}$, em que $\left(\mathbf{A}_{k}\right)_{\mathbb{S}, \overline{\mathbb{S}}}$ é a matriz de dimensão $|\mathbb{S}| \times|\overline{\mathbb{S}}|$ formada pelas linhas de $\mathbf{A}_{k}$ cujos índices pertencem a $\mathbb{S}$ e pelas colunas de $\mathbf{A}_{k}$ cujos índices pertencem a $\overline{\mathbb{S}}$. Logo, a estimativa esparsa associada ao suporte $\mathbb{S}$, no instante $k$, pode ser expressa como

$$
\hat{\mathbf{h}}_{k}^{\mathbb{S}}=(\mathbf{h})_{\mathbb{S}}+\boldsymbol{v}_{k}^{\mathbb{S}}+\boldsymbol{\varepsilon}_{k}^{\mathbb{S}}
$$

em que $\boldsymbol{v}_{k}^{\mathbb{S}}$ é aqui denominado desvio-cruzado, definido como

$$
\boldsymbol{v}_{k}^{\mathbb{S}} \triangleq\left(\mathbf{A}_{k}\right)_{\mathbb{S}, \mathbb{S}}^{-1}\left(\mathbf{A}_{k}\right)_{\mathbb{S}, \overline{\mathbb{S}}}(\mathbf{h})_{\overline{\mathbb{S}}}
$$

e $\varepsilon_{k}^{\mathbb{S}}$ é aqui denominado desvio-de-ruído, definido como

$$
\boldsymbol{\varepsilon}_{k}^{\mathbb{S}} \triangleq\left(\mathbf{A}_{k}\right)_{\mathbb{S}, \mathbb{S}}^{-1}\left[\frac{1}{k} \sum_{i=1}^{k}\left(\mathbf{C}_{i}\right)_{\mathbb{S}}^{H} \mathbf{z}_{i}\right]
$$

O desvio-cruzado em 3-18 também pode ser alternativamente expresso como 


$$
\boldsymbol{v}_{k}^{\mathbb{S}}=\left(\mathbf{A}_{k}\right)_{\mathbb{S}, \mathbb{S}}^{-1}\left(\mathbf{A}_{k}\right)_{\mathbb{S}, \tilde{\mathbb{S}}}(\mathbf{h})_{\tilde{\mathbb{S}}}
$$

em que

$$
\tilde{\mathbb{S}}=\mathbb{S}_{\mathbf{h}} \backslash \mathbb{S}=\mathbb{S}_{\mathbf{h}} \cap \overline{\mathbb{S}}
$$

é o conjunto dos elementos de $\mathbb{S}_{\mathbf{h}}$ que não estão em $\mathbb{S}$. Portanto, se $\mathbb{S}=\mathbb{L}$, então $\overline{\mathbb{S}}=\emptyset$ e, consequentemente, $\boldsymbol{v}_{k}^{\mathbb{S}}$ não existe. Similarmente, se $\mathbb{L} \supset \mathbb{S} \supseteq \mathbb{S}_{\mathbf{h}}$, então $(\mathbf{h})_{\tilde{\mathbb{S}}}=\mathbf{0}$ e, consequentemente, $\boldsymbol{v}_{k}^{\mathbb{S}}=\mathbf{0}$.

Em suma, 3-17 mostra que a estimativa $\hat{\mathbf{h}}_{k}^{\mathbb{S}}$ pode ser expressa como a soma de três parcelas. A primeira parcela $(\mathbf{h})_{\mathbb{S}}$ corresponde ao vetor formado pelos elementos da resposta ao impulso verdadeira $\mathbf{h}$ cujos índices pertencem a S. A segunda parcela $\boldsymbol{v}_{k}^{\mathbb{S}}$ é denominada desvio-cruzado e seus elementos são formados pela combinação linear dos elementos de $\mathbf{h}$ cujos índices não pertencem a $\mathbb{S}$. A terceira parcela $\varepsilon_{k}^{\mathbb{S}}$ é denominada desvio-de-ruído e seus elementos são formados pela combinação linear dos elementos do vetor $\mathbf{z}_{k}$ que representa o ruído mais interferência.

\section{4}

\section{Análise de convergência das estimativas LS com suporte reduzido}

Suponha que o sinal na entrada do sistema estimado possa ser representado por uma sequência de vetores pseudoaleatórios $\mathbf{c}_{k}$ de média nula e matriz autocorrelação $\left(\mathcal{E}_{c} / N\right) \mathbf{I}$, para todo $k$ e com $\mathcal{E}_{c}<\infty$. Sem perda de generalidade, deste ponto em diante é admitido que $\mathcal{E}_{c}=1$. Nesse caso, com base na forma forte da Lei dos Grandes Números, é possível verificar que, quando $k$ tende a infinito, a matriz $\mathbf{A}_{k}$ converge com probabilidade 1 para a matriz-identidade, isto é,

$$
\operatorname{Pr}\left[\lim _{k \rightarrow \infty} \mathbf{A}_{k}=\mathbf{I}\right]=1
$$

Em decorrência disso, é possível demonstrar que, quando $k$ tende a infinito, a inversa da matriz $\mathbf{A}_{k}$ também converge com probabilidade 1 para a inversa do limite da matriz $\mathbf{A}_{k}$, ou seja,

$$
\operatorname{Pr}\left[\lim _{k \rightarrow \infty} \mathbf{A}_{k}^{-1}=\left(\lim _{k \rightarrow \infty} \mathbf{A}_{k}\right)^{-1}=\mathbf{I}\right]=1
$$

Também devido à Lei Forte dos Grandes Números, é possível afirmar que

$$
\operatorname{Pr}\left[\lim _{k \rightarrow \infty}\left(\mathbf{A}_{k}\right)_{\mathbb{S}, \mathbb{S}}^{-1}=\left(\lim _{k \rightarrow \infty}\left(\mathbf{A}_{k}\right)_{\mathbb{S}, \mathbb{S}}\right)^{-1}=\mathbf{I}^{-1}=\mathbf{I}\right]=1
$$


e que

$$
\operatorname{Pr}\left[\lim _{k \rightarrow \infty}\left(\mathbf{A}_{k}\right)_{\mathbb{S}, \overline{\mathbb{S}}}=\left(\lim _{k \rightarrow \infty} \mathbf{A}_{k}\right)_{\mathbb{S}, \overline{\mathbb{S}}}=(\mathbf{I})_{\mathbb{S}, \overline{\mathbb{S}}}=\mathbf{0}\right]=1
$$

Aplicando 3-24 e 3-25 em 3-18, conclui-se então que

$$
\operatorname{Pr}\left[\lim _{k \rightarrow \infty} \boldsymbol{v}_{k}^{\mathbb{S}}=\mathbf{0}\right]=1 \forall \mathbb{S}
$$

Aplicando 3-24 em 3-19 e supondo que cada elemento de $\mathbf{z}_{i}$ seja variável aleatória de média zero e variância $\sigma_{z}^{2}<\infty$, todos estatisticamente independentes entre si, então verifica-se que

$$
\operatorname{Pr}\left[\lim _{k \rightarrow \infty} \varepsilon_{k}^{\mathbb{S}}=\mathbf{0}\right]=1 \forall \mathbb{S}
$$

Ou seja, verifica-se que, para qualquer suporte $\mathbb{S}$, tanto o desvio-cruzado $\boldsymbol{v}_{k}^{\mathbb{S}}$ quanto o desvio-de-ruído $\varepsilon_{k}^{\mathbb{S}}$ tendem a zero com probabilidade 1 quando $k$ tende a infinito.

Juntando 3-17, 3-26 e 3-27, conclui-se que

$$
\operatorname{Pr}\left[\lim _{k \rightarrow \infty} \hat{\mathbf{h}}_{k}^{\mathbb{S}}=(\mathbf{h})_{\mathbb{S}}\right]=1 \forall \mathbb{S}
$$

Portanto, verifica-se que $\mathbf{h}_{k}^{\mathbb{S}}$ consiste em uma estimativa consistente e despolarizada de $(\mathbf{h})_{\mathbb{S}}$.

Seja $\hat{\mathbf{h}}_{k}$ a estimativa LS esparsa do sistema no instante $k$, dada por

$$
\hat{\mathbf{h}}_{k}=\mathbf{I}_{\mathbb{L}, \hat{\mathbb{S}}} \hat{\mathbf{h}}_{k}^{\hat{\mathbb{S}}}
$$

em que $\hat{\mathbb{S}}$ é a estimativa do suporte $\mathbb{S}_{\mathbf{h}}$. A equação 3-28 mostra que se $\widehat{\mathbb{S}} \supseteq \mathbb{S}_{\mathbf{h}}$, então $\hat{\mathbf{h}}_{k}$ converge com probabilidade 1 para a resposta ao impulso verdadeira do sistema $\mathbf{h}$.

A Figura 3.1 ilustra a convergência da estimação LS com suporte reduzido de um sistema $\operatorname{MESS}(100,10)$ (o modelo MESS é descrito no Apêndice B), com $\mathcal{E}_{\text {chip }} / \sigma_{z}^{2}=5 \mathrm{~dB}$, em função da cardinalidade de $\mathbb{S}$, para os seguintes casos particulares:

- $\mathbb{S}=\mathbb{L}$, que corresponde à estimação LS full-support ordinária;

- $\mathbb{S}=\mathbb{S}_{\mathbf{h}}$, que corresponde à estimação LS Oráculo (com conhecimento a priori do suporte verdadeiro do sistema);

$-\mathbb{S} \supset \mathbb{S}_{\mathbf{h}}, \operatorname{com}|\mathbb{S}|=20$.

$-\mathbb{S} \supset \mathbb{S}_{\mathbf{h}}, \operatorname{com}|\mathbb{S}|=40$. 


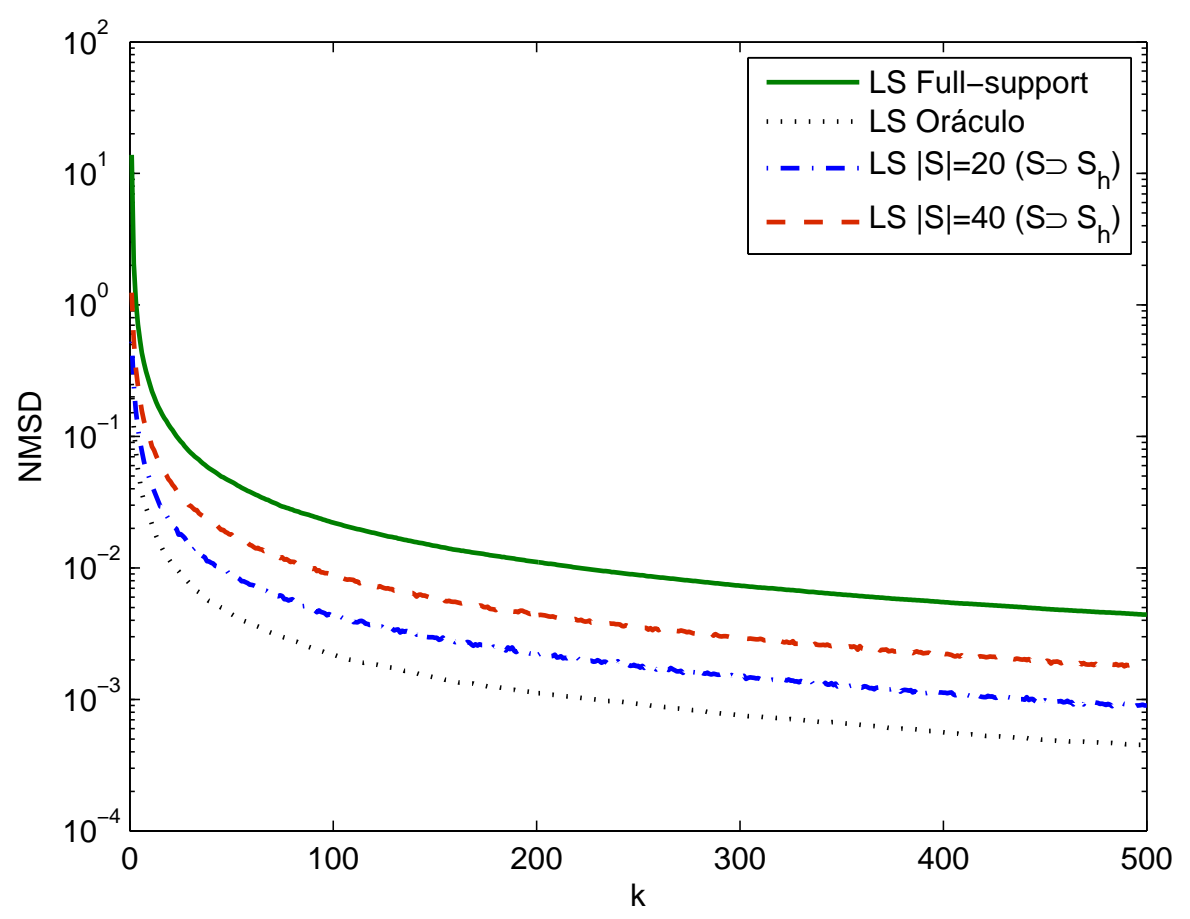

Figura 3.1: Convergência de estimativas LS de um sistema $\operatorname{MESS}(100,10)$, $\operatorname{com} \mathcal{E}_{\text {chip }} / \sigma_{z}^{2}=5 \mathrm{~dB}, \mathrm{~N}=16$ e $\mathrm{Q}=100$, em função do quantidade $k$ de blocos processados

A convergência da estimação LS na Figura 3.1 é mostrada em função do Desvio Médio Quadrático Normalizado (Normalized Mean Square Deviation - NMSD), calculado como

$$
\operatorname{NMSD}(k)=\frac{1}{Q} \sum_{q=1}^{Q} \frac{\left\|\hat{\mathbf{h}}^{(q)}-\mathbf{h}^{(q)}\right\|^{2}}{\left\|\mathbf{h}^{(q)}\right\|^{2}}
$$

em que $Q$ corresponde à quantidade de realizações do sistema e $(.)^{(q)}$ corresponde à $q$-ésima realização. Nessa simulação, foram geradas $Q=100$ realizações do sistema. O tamanho das sequências pseudoaleatórias na entrada do sistema foi $N=16$. Os elementos de cada realização $\mathbb{S}^{(q)}$ não-pertencentes ao suporte de $\mathbf{h}^{(q)}$ foram sorteados tendo todos a mesma probabilidade.

A Figura 3.1 ilustra o fato de que, quando $\mathbb{S}$ contém $\mathbb{S}_{\mathbf{h}}$, quanto menor a cardinalidade de $\mathbb{S}$, mais rápida a convergência da estimação LS com suporte reduzido. Portanto, um dos principais objetivos da estimação LS com suporte reduzido é acelerar a convergência no processo de estimação da resposta ao impulso discreta de sistemas esparsos, restringindo a estimação LS a um suporte $\mathbb{S}$ escolhido de forma adequada. 


\section{5}

\section{Análise de convergência do custo LS com suporte reduzido}

A equação 3-28 mostra que $\hat{\mathbf{h}}_{k}^{\mathbb{S}}$ converge com probabilidade 1 para $(\mathbf{h})_{\mathbb{S}}$ quando $k \rightarrow \infty$ e é repetida a seguir com outra notação

$$
\hat{\mathbf{h}}_{k}^{\mathbb{S}} \stackrel{a . s .}{\longrightarrow}(\mathbf{h})_{\mathbb{S}}
$$

Portanto, tem-se que

$$
J_{k}^{\mathbb{S}}\left(\hat{\mathbf{h}}_{k}^{\mathbb{S}}\right) \stackrel{a . s}{\longrightarrow} J_{k}^{\mathbb{S}}\left((\mathbf{h})_{\mathbb{S}}\right)
$$

Nesta seção, é realizada a análise de convergência de $J_{k}^{\mathbb{S}}\left(\hat{\mathbf{h}}_{k}^{\mathbb{S}}\right)$, quando $k$ tende a infinito, considerando duas hipóteses:

- Primeira hipótese: $\mathbb{S}$ é superconjunto de $\mathbb{S}_{\mathbf{h}}$;

- Segunda hipótese: $\mathbb{S}$ não é superconjunto de $\mathbb{S}_{\mathbf{h}}$.

\section{Primeira hipótese: $\mathbb{S}$ é superconjunto de $\mathbb{S}_{h}$}

Suponha que $\mathbb{S} \supseteq \mathbb{S}_{\mathbf{h}}$. Nesse caso, $\left(\mathbf{C}_{i}\right)_{\mathbb{S}}(\mathbf{h})_{\mathbb{S}}=\mathbf{C}_{i} \mathbf{h}$. Dessa forma,

$$
J_{k}^{\mathbb{S}}\left((\mathbf{h})_{\mathbb{S}}\right)=\frac{1}{k} \sum_{i=1}^{k}\left\|\mathbf{r}_{i}-\left(\mathbf{C}_{i}\right)_{\mathbb{S}}(\mathbf{h})_{\mathbb{S}}\right\|^{2}=\frac{1}{k} \sum_{i=1}^{k}\left\|\mathbf{r}_{i}-\mathbf{C}_{i} \mathbf{h}\right\|^{2}=J_{k}^{\mathbb{L}}(\mathbf{h})
$$

Aplicando 3-1 em 3-33, tem-se que

$$
J_{k}^{\mathbb{L}}(\mathbf{h})=\frac{1}{k} \sum_{i=1}^{k}\left\|\mathbf{z}_{i}\right\|^{2}
$$

Supondo que $\left\{\mathbf{z}_{i}, i=1,2, \ldots\right\}$ seja um processo ergódico no tempo (nos instantes $i$ ) e que os elementos de $\mathbf{z}_{i}$ sejam variáveis aleatórias independentes entre si de média zero e variância $\sigma_{z}^{2}$, então é possível afirmar que

$$
\lim _{k \rightarrow \infty} J_{k}^{\mathbb{L}}(\mathbf{h})=\lim _{k \rightarrow \infty} \frac{1}{k} \sum_{i=1}^{k}\left\|\mathbf{z}_{i}\right\|^{2}=E\left\{\left\|\mathbf{z}_{i}\right\|^{2}\right\}=M \sigma_{z}^{2} \quad \forall i
$$

Portanto, aplicando 3-35 e 3-33 em 3-32, conclui-se que

$$
J_{k}^{\mathbb{S}}\left(\hat{\mathbf{h}}_{k}^{\mathbb{S}}\right) \stackrel{a . s .}{\longrightarrow} M \sigma_{z}^{2} \quad \mathbb{S} \supseteq \mathbb{S}_{\mathbf{h}}
$$

Em suma, se $\mathbb{S}$ é um superconjunto de $\mathbb{S}_{\mathbf{h}}$, então $J_{k}^{\mathbb{S}}\left(\hat{\mathbf{h}}_{k}^{\mathbb{S}}\right)$ converge com probabilidade 1 para o patamar de ruído-mais-interferência $M \sigma_{z}^{2}$. 


\section{Segunda hipótese: $\mathbb{S}$ não é superconjunto de $\mathbb{S}_{\mathrm{h}}$}

Suponha que $\mathbb{S} \nsupseteq \mathbb{S}_{\mathbf{h}}$. Nesse caso, $\tilde{\mathbb{S}} \neq \emptyset$ e, portanto, $\left(\mathbf{C}_{i}\right)_{\mathbb{S}}(\mathbf{h})_{\mathbb{S}}=\mathbf{C}_{i} \mathbf{h}-$ $\left(\mathbf{C}_{i}\right)_{\tilde{\mathbb{S}}}(\mathbf{h})_{\tilde{\mathbb{S}}}$. Logo, verifica-se que

$$
J_{k}^{\mathbb{S}}\left((\mathbf{h})_{\mathbb{S}}\right)=\frac{1}{k} \sum_{i=1}^{k}\left\|\mathbf{r}_{i}-\left(\mathbf{C}_{i} \mathbf{h}-\left(\mathbf{C}_{i}\right)_{\tilde{\mathbb{S}}}(\mathbf{h})_{\tilde{\mathbb{S}}}\right)\right\|^{2}
$$

Utilizando 3-1, 3-37 pode ser reescrita da seguinte forma

$$
J_{k}^{\mathbb{S}}\left((\mathbf{h})_{\mathbb{S}}\right)=\frac{1}{k} \sum_{i=1}^{k}\left\|\mathbf{z}_{i}+\left(\mathbf{C}_{i}\right)_{\tilde{\mathbb{S}}}(\mathbf{h})_{\tilde{\mathbb{S}}}\right\|^{2}
$$

que pode ser expandida como

$$
J_{k}^{\mathbb{S}}\left((\mathbf{h})_{\mathbb{S}}\right)=\frac{1}{k} \sum_{i=1}^{k}\left\{\left\|\mathbf{z}_{i}\right\|^{2}+2 \Re\left[\mathbf{z}_{i}^{H}\left(\mathbf{C}_{i}\right)_{\tilde{\mathbb{S}}}(\mathbf{h})_{\tilde{\mathbb{S}}}\right]+\left\|\left(\mathbf{C}_{i}\right)_{\tilde{\mathbb{S}}}(\mathbf{h})_{\tilde{\mathbb{S}}}\right\|^{2}\right\}
$$

Por hipótese, $\left\{\mathbf{z}_{i}, i=1,2, \ldots\right\}$ é um processo ergódico, em que os elementos de $\mathbf{z}_{i}$ são variáveis aleatórias independentes entre si de média zero e variância $\sigma_{z}^{2},\left\{\mathbf{c}_{i}, i=1,2, \ldots\right\}$ é processo ergódico de média zero, com $\mathcal{E}_{c}=E\left\{\left\|\mathbf{c}_{i}\right\|^{2}\right\}$, e os dois processos são independentes entre si. Nesse caso, quando $k$ tende a infinito, 3-39 pode ser reescrita como

$$
\lim _{k \rightarrow \infty} J_{k}^{\mathbb{S}}\left((\mathbf{h})_{\mathbb{S}}\right)=M \sigma_{z}^{2}+\mathcal{E}_{c}\left\|(\mathbf{h})_{\tilde{S}}\right\|^{2} \quad \tilde{\mathbb{S}} \neq \emptyset
$$

Aplicando 3-40 em 3-32, tem-se que

$$
J_{k}^{\mathbb{S}}\left(\hat{\mathbf{h}}_{k}^{\mathbb{S}}\right) \stackrel{a . s .}{\longrightarrow} M \sigma_{z}^{2}+\mathcal{E}_{c}\left\|(\mathbf{h})_{\tilde{\mathbb{S}}}\right\|^{2} \quad \tilde{\mathbb{S}} \neq \emptyset
$$

A razão sinal-ruído-mais-interferência média (SINR) é definida como

$$
\mathrm{SINR} \triangleq \frac{\mathcal{E}_{c} E\left[\|\mathbf{h}\|^{2}\right]}{\sigma_{z}^{2}}
$$

Lembrando que, por hipótese, o sistema é considerado invariante no tempo ao longo de todo o processo de estimação (e, nesse caso, $E\left[\|\mathbf{h}\|^{2}\right]=\|\mathbf{h}\|^{2}$ ), então a SINR, dado $\mathbf{h}$, pode ser reescrita como

$$
\operatorname{SINR}=\frac{\mathcal{E}_{c}\|\mathbf{h}\|^{2}}{\sigma_{z}^{2}}
$$

Aplicando 3-43 em 3-41, conclui-se que 


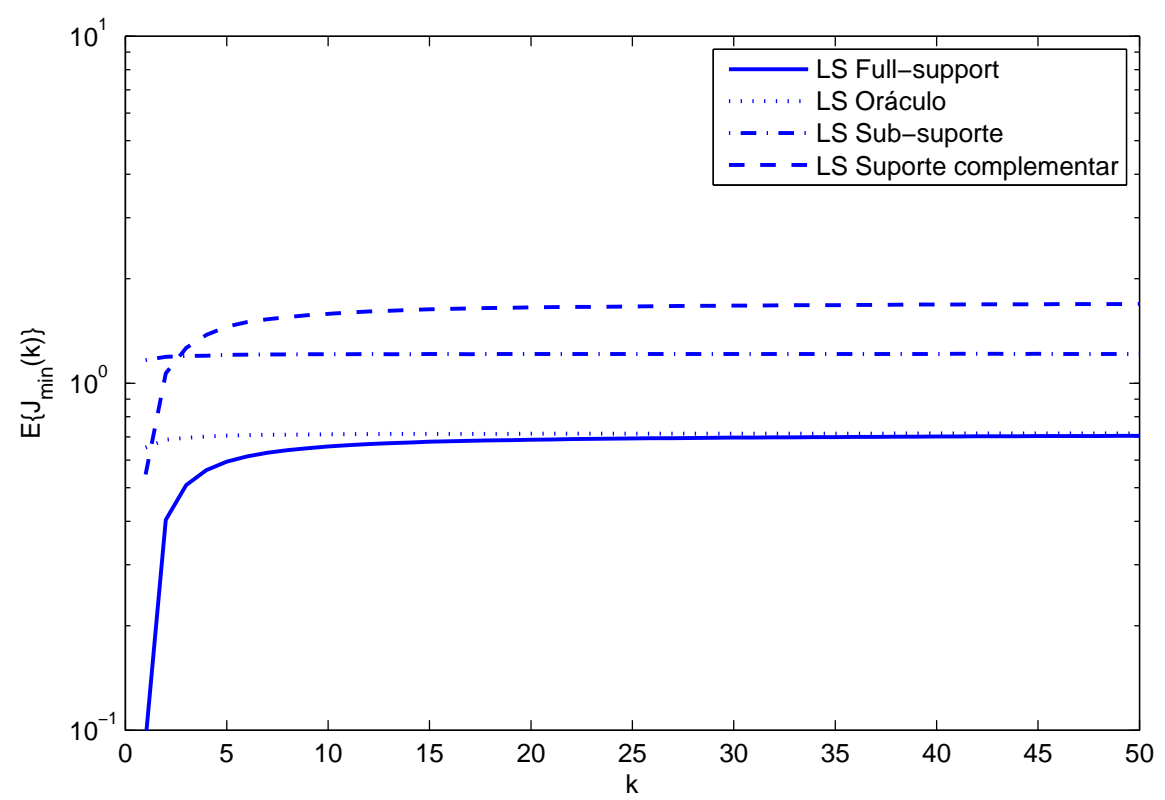

Figura 3.2: Curvas de convergência do custo LS para $\mathcal{E}_{\text {chip }} / \sigma_{z}^{2}=10 \mathrm{~dB}$ e $\mathrm{N}=16$, avaliada em $\mathrm{Q}=1000$ realizações de um sistema $\operatorname{MESS}(100,10)$

$$
J_{k}^{\mathbb{S}}\left(\hat{\mathbf{h}}_{k}^{\mathbb{S}}\right) \stackrel{\text { a.s. }}{\longrightarrow}\left[1+\frac{\mathrm{SINR}}{M} \frac{\left\|(\mathbf{h})_{\tilde{\mathbb{S}}}\right\|^{2}}{\|\mathbf{h}\|^{2}}\right] M \sigma_{z}^{2} \quad \tilde{\mathbb{S}} \neq \emptyset
$$

Em suma, se $\mathbb{S}$ não contém $\mathbb{S}_{\mathbf{h}}$, então quanto maior for o produto entre a razão sinal-ruído-mais-interferência (SINR) e a razão entre as energias de $(\mathbf{h})_{\tilde{\mathbb{S}}}$ e $\mathbf{h}$, maior será a diferença entre $J_{k}^{\mathbb{S}}\left(\hat{\mathbf{h}}_{k}^{\mathbb{S}}\right)$ e o patamar de ruído-mais-interferência $M \sigma_{z}^{2}$ quando $k$ tende a infinito. Convém observar que, se $\mathbb{S} \supseteq \mathbb{S}_{\mathbf{h}}$, então $\widetilde{\mathbb{S}}=\emptyset$ e 3-44 degenera-se para o formato de 3-36.

A Figura 3.2 ilustra a evolução de $E\left\{J_{k}(\mathbb{S})\right\}$ em função de $k$, avaliada em $\mathrm{Q}=1000$ realizações de um sistema $\operatorname{MESS}(100,10)$, com $\mathcal{E}_{\text {chip }} / \sigma_{z}^{2}=10 \mathrm{~dB}$ e $N=$ 16, para quatro casos particulares de $\mathbb{S}$ : a curva LS Full-support corresponde a $\mathbb{S}=\mathbb{L} ;$ a curva LS Oráculo corresponde ao caso ideal em que $\mathbb{S}=\mathbb{S}_{\mathbf{h}} ;$ a curva LS Sub-suporte corresponde ao caso indesejado em que $\mathbb{S}$ é subconjunto de $\mathbb{S}_{\mathbf{h}}$, particularmente aqui $\mathbb{S}=\left\{s_{i} \mid s_{i} \in \mathbb{S}_{\mathbf{h}}, i=1 \ldots S / 2\right.$ e $\left.\left|h_{s_{i}}\right|>\left|h_{s_{i+1}}\right|\right\}$; e LS Suporte complementar corresponde ao caso indesejado em que $\mathbb{S}=\mathbb{L} \backslash \mathbb{S}_{\mathbf{h}}$. Conforme esperado, observa-se a convergência das curvas LS Full-support e LS Oráculo para o patamar de ruído-mais-interferência, mas com a diferença da estimação LS Oráculo convergir mais rapidamente, pelo fato de que $\mathbb{S}_{\mathbf{h}} \subseteq \mathbb{L}$. Por outro lado, como também era esperado, verifica-se uma severa degradação de desempenho nas estimativas LS Sub-suporte e LS Suporte complementar, uma vez que, nesses dois casos, $\mathbb{S}$ não é superconjunto de $\mathbb{S}_{\mathbf{h}}$. 


\section{6}

\section{O erro médio quadrático como medida de distância}

A equação 3-1 pode ser reescrita na seguinte forma ampliada

$$
\overline{\mathbf{r}}_{k}=\overline{\mathbf{C}}_{k} \mathbf{h}+\overline{\mathbf{z}}_{k}
$$

em que $\overline{\mathbf{r}}_{k}=\left[\mathbf{r}_{1}^{T} \mathbf{r}_{2}^{T} \ldots \mathbf{r}_{k}^{T}\right]^{T}$ é vetor de dimensão $k M \times 1, \overline{\mathbf{C}}_{k}=\left[\mathbf{C}_{1}^{T} \mathbf{C}_{2}^{T} \ldots \mathbf{C}_{k}^{T}\right]^{T}$ é matriz de dimensão $k M \times L$ e $\overline{\mathbf{z}}_{k}=\left[\mathbf{z}_{1}^{T} \mathbf{z}_{2}^{T} \ldots \mathbf{z}_{k}^{T}\right]^{T}$ é vetor de dimensão $k M \times 1$. Quando $\mathbb{S} \supseteq \mathbb{S}_{\mathbf{h}}, 3-45$ pode ser reescrita como

$$
\overline{\mathbf{r}}_{k}=\left(\overline{\mathbf{C}}_{k}\right)_{\mathbb{S}}(\mathbf{h})_{\mathbb{S}}+\overline{\mathbf{z}}_{k}
$$

em que $\left(\overline{\mathbf{C}}_{k}\right)_{\mathbb{S}}$ é a matriz formada pelas colunas de $\overline{\mathbf{C}}_{k}$ cujos índices pertencem a $\mathbb{S}$.

Observando 3-46, a função-custo $J_{k}^{\mathbb{S}}(\mathbf{x})$, dada por 3-5, que fornece a média aritmética da soma dos erros quadráticos associada ao suporte $\mathbb{S}$, pode ser reescrita da seguinte forma

$$
J_{k}^{\mathbb{S}}(\mathbf{x})=\frac{1}{k}\left\|\overline{\mathbf{r}}_{k}-\left(\overline{\mathbf{C}}_{k}\right)_{\mathbb{S}} \mathbf{x}\right\|^{2}
$$

O vetor $\hat{\mathbf{h}}_{k}^{\mathbb{S}}$ que minimiza 3-47 é dado por

$$
\hat{\mathbf{h}}_{k}^{\mathbb{S}}=\left(\overline{\mathbf{C}}_{k}\right)_{\mathbb{S}}^{\dagger} \overline{\mathbf{r}}_{k}=\left(\left(\overline{\mathbf{C}}_{k}\right)_{\mathbb{S}}^{H}\left(\overline{\mathbf{C}}_{k}\right)_{\mathbb{S}}\right)^{-1}\left(\overline{\mathbf{C}}_{k}\right)_{\mathbb{S}}^{H} \overline{\mathbf{r}}_{k}
$$

que pode ser reescrita como

$$
\hat{\mathbf{h}}_{k}^{\mathbb{S}}=\left(\frac{1}{k} \sum_{i=1}^{k}\left(\mathbf{C}_{i}\right)_{\mathbb{S}}^{H}\left(\mathbf{C}_{i}\right)_{\mathbb{S}}\right)^{-1}\left(\frac{1}{k} \sum_{i=1}^{k}\left(\mathbf{C}_{i}\right)_{\mathbb{S}}^{H} \mathbf{r}_{i}\right)=\left(\mathbf{A}_{k}\right)_{\mathbb{S}, \mathbb{S}}^{-1}\left(\mathbf{b}_{k}\right)_{\mathbb{S}}
$$

Coerentemente, os resultados em 3-48 e 3-49 são o mesmo que em 3-9.

Seja $\mathbb{U}_{k}$ o espaço-vetorial contendo todos os possíveis valores de $\overline{\mathbf{r}}_{k}$ (isto é, o espaço-vetorial varrido pelos vetores da base canônica formada pelas colunas da matriz-identidade $\left.\mathbf{I}_{k M \times k M}\right)$. Seja também $\mathbb{V}_{k}^{\mathbb{S}}$ o espaço-vetorial varrido pelas colunas de $\left(\overline{\mathbf{C}}_{k}\right)_{\mathbb{S}}$. Como a cardinalidade de $\mathbb{S}$ é menor ou igual à dimensão de $\mathbb{U}_{k}$, então $\mathbb{V}_{k}^{\mathbb{S}}$ constitui-se em um sub-espaço de $\mathbb{U}_{k}$. A equação 3-47 mostra que $\overline{\mathbf{s}}_{k}^{\mathbb{S}}=\left(\overline{\mathbf{C}}_{k}\right)_{\mathbb{S}} \hat{\mathbf{h}}_{k}^{\mathbb{S}}$ representa o vetor pertencente ao sub-espaço $\mathbb{V}_{k}^{\mathbb{S}}$ mais próximo (com menor distância Euclidiana) do vetor de observação $\overline{\mathbf{r}}_{k}$, sendo que $\hat{\mathbf{h}}_{k}^{\mathbb{S}}$ representa as coordenadas de $\overline{\mathbf{s}}_{k}^{\mathbb{S}}$ em relação à base formada pelas colunas de $\left(\overline{\mathbf{C}}_{k}\right)_{\mathbb{S}}$. Como $J_{k}^{\mathbb{S}}($.$) é função convexa, então o vetor \hat{\mathbf{h}}_{k}^{\mathbb{S}}$ que minimiza $J_{k}^{\mathbb{S}}($.$) é$ 
único. Portanto, o elemento $\overline{\mathbf{s}}_{k}^{\mathbb{S}}$, pertencente ao sub-espaço $\mathbb{V}_{k}^{\mathbb{S}}$, mais próximo de $\overline{\mathbf{r}}_{k}$, é único. A unicidade de $\overline{\mathbf{s}}_{k}^{\mathbb{S}}$ permite demonstrar o teorema a seguir.

Teorema 3.1 Se $\mathbb{S}_{1} \supset \mathbb{S}_{2}$, então $J_{k}\left(\mathbb{S}_{1}\right) \leq J_{k}\left(\mathbb{S}_{2}\right)$

Prova. Sejam dois suportes $\mathbb{S}_{1}$ e $\mathbb{S}_{2}$ tais que $\mathbb{S}_{1} \supset \mathbb{S}_{2}$. Nesse caso, o subespaço $\mathbb{V}_{k}^{\mathbb{S}_{1}}$ contém o sub-espaço $\mathbb{V}_{k}^{\mathbb{S}_{2}}$. Sejam também $\overline{\mathbf{s}}_{k}^{\mathbb{S}_{1}}$ e $\overline{\mathbf{s}}_{k}^{\mathbb{S}_{2}}$ os elementos pertencentes, respectivamente, a $\mathbb{V}_{k}^{\mathbb{S}_{1}} e \mathbb{V}_{k}^{\mathbb{S}_{2}}$ que apresentam a menor distância Euclidiana em relação a $\overline{\mathbf{r}}_{k}$. Se $\overline{\mathbf{s}}_{k}^{\mathbb{S}_{1}}$ pertence a $\mathbb{V}_{k}^{\mathbb{S}_{2}}$ então, como $\overline{\mathbf{s}}_{k}^{\mathbb{S}_{2}}$ é único, obrigatoriamente tem-se que $\overline{\mathbf{s}}_{k}^{\mathbb{S}_{1}}=\overline{\mathbf{s}}_{k}^{\mathbb{S}_{2}}$ e, consequentemente, $J_{k}\left(\mathbb{S}_{1}\right)=J_{k}\left(\mathbb{S}_{2}\right)$. Por outro lado, se $\overline{\mathbf{s}}_{k}^{\mathbb{S}_{1}}$ não pertence $a \mathbb{V}_{k}^{\mathbb{S}_{2}}$, então a distância Euclidiana entre $\overline{\mathbf{s}}_{k}^{\mathbb{S}_{1}}$ e $\overline{\mathbf{r}}_{k}$ é, obrigatoriamente (devido à definição de $\overline{\mathbf{s}}_{k}^{\mathbb{S}_{1}}$ ), menor que a distância Euclidiana entre $\overline{\mathbf{s}}_{k}^{\mathbb{S}_{2}}$ e $\overline{\mathbf{r}}_{k}$ e, nesse caso, $J_{k}\left(\mathbb{S}_{1}\right)<J_{k}\left(\mathbb{S}_{2}\right)$. Logo, fica assim demonstrado que, se $\mathbb{S}_{1} \supset \mathbb{S}_{2}$, então $J_{k}\left(\mathbb{S}_{1}\right) \leq J_{k}\left(\mathbb{S}_{2}\right)$.

O fato de que, se $\mathbb{S}_{1} \supset \mathbb{S}_{2}$, então $J_{k}\left(\mathbb{S}_{1}\right) \leq J_{k}\left(\mathbb{S}_{2}\right)$, pode também ser explicado da seguinte forma: as dimensões extras do espaço-vetorial $\mathbb{V}_{k}^{\mathbb{S}_{1}}$ em relação ao seu sub-espaço $\mathbb{V}_{k}^{\mathbb{S}_{2}}$ conferem um maior grau de liberdade à equação 3-47, tendo como consequência $\left\|\overline{\mathbf{r}}_{k}-\overline{\mathbf{s}}_{k}^{\mathbb{S}_{1}}\right\| \leq\left\|\overline{\mathbf{r}}_{k}-\overline{\mathbf{s}}_{k}^{\mathbb{S}_{2}}\right\|$.

A Figura 3.3 mostra a variação de $J_{k}(\mathbb{S})$, no instante $k=100$, em função de $\mathbb{S}$ para uma única realização de um sistema $\operatorname{MESS}(100,10), \operatorname{com} \mathcal{E}_{\text {chip }} / \sigma_{z}^{2}=10 \mathrm{~dB}$ e $\mathrm{N}=16$. Nesse experimento, iniciou-se com $\mathbb{S}_{1}=\mathbb{L}$ e gerou-se uma sequência $\left\{\mathbb{S}_{i} \supset \mathbb{S}_{i+1}\right\}$ retirando-se aleatoriamente um elemento de $\mathbb{S}_{i}$ para a determinação de $\mathbb{S}_{i+1}$. Conforme esperado, observa-se que a sequência $\left\{J_{k}\left(\mathbb{S}_{i}\right) \mid \mathbb{S}_{i} \supset\right.$ $\left.\mathbb{S}_{i+1}\right\}$ é monótona não-decrescente. Os saltos mais acentuados correspondem à retirada em $\mathbb{S}_{i}$ de algum elemento pertencente a $\mathbb{S}_{\mathbf{h}}$.

Qualquer estratégia de estimação de sistemas esparsos inevitavelmente induz um relaxamento do custo LS, no sentido em que a estimativa LS esparsa não coincide com a estimativa LS full-support ordinária, que é a que apresenta o menor custo LS. O Teorema 3.1, demonstrado nesta seção, vai um passo além ao mostrar que qualquer sequência $\left\{J_{k}\left(\mathbb{S}_{i}\right) \mid \mathbb{S}_{i+1} \subset \mathbb{S}_{i}\right\}$ é monótona não-decrescente.

\section{7}

\section{Determinação da estimativa LS através de algoritmos de busca}

Observando 3-9, constata-se que o cálculo de $\hat{\mathbf{h}}_{k}^{\mathbb{S}}$ envolve a inversão da matriz $\left(\mathbf{A}_{k}\right)_{\mathbb{S}, \mathbb{S}}$. Como será visto no próximo capítulo, a estratégia de Encolhimentos Sucessivos inicia-se com o cálculo de $\hat{\mathbf{h}}_{k}^{\mathbb{L}}$, o que torna o armazenamento em memória de $\mathbf{A}_{k}^{-1}$, previamente calculado, conveniente. Entretanto, para 


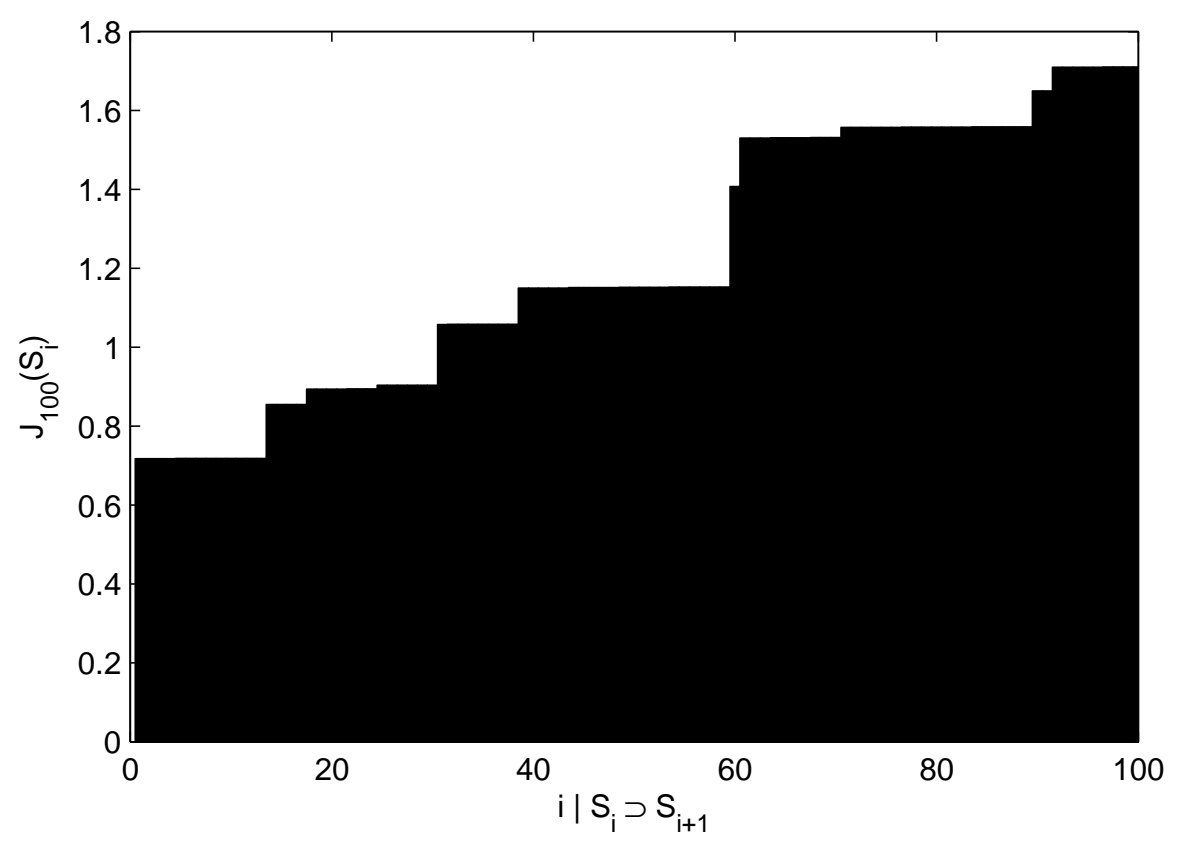

Figura 3.3: Monotonicidade de uma sequência $\left\{J_{k}\left(\mathbb{S}_{i}\right) \mid \mathbb{S}_{i} \supset \mathbb{S}_{i+1}\right\}$, em $\mathrm{k}=100$, gerada aleatoriamente, referente a uma única realização de um sistema $\operatorname{MESS}(100,10), \operatorname{com} \mathcal{E}_{\text {chip }} / \sigma_{z}^{2}=10 \mathrm{~dB}$ e $\mathrm{N}=16$

$\mathbb{S} \neq \mathbb{L}$, torna-se ser impraticável o pré-armazenamento em memória de todas as matrizes $\left(\mathbf{A}_{k}\right)_{\mathbb{S}, \mathbb{S}}^{-1}$ correspondentes a todas as possibilidades de $\mathbb{S} \subseteq \mathbb{L}$, com $\mathbb{S} \neq \emptyset$.

Uma alternativa a 3-9 em que se evita o cálculo online de $\left(\mathbf{A}_{k}\right)_{\mathbb{S}, \mathbb{S}}^{-1}$ é a busca iterativa de $\hat{\mathbf{h}}_{k}^{\mathbb{S}}$ através de Algoritmos de Descida (Descent Algorithms) (28), que podem ser expressos conforme o Algoritmo 3.1.

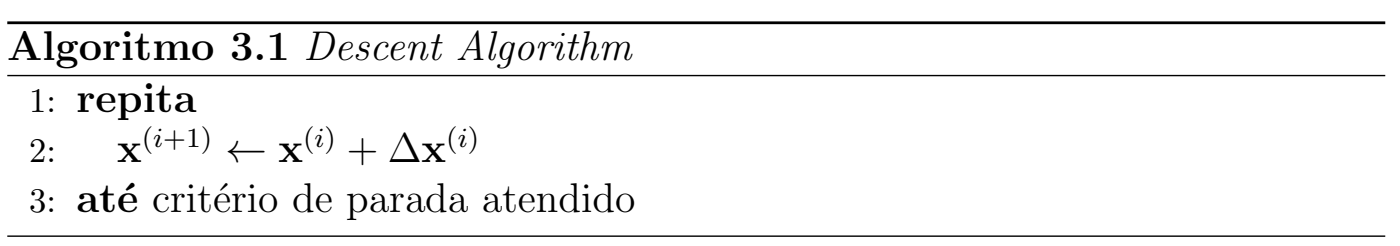

em que $\mathbf{x}^{(i)}$ representa o vetor resultante da busca e $\Delta \mathbf{x}^{(i)}$ é o vetor de busca, ambos referentes à $i$-ésima iteração. O vetor de busca é escolhido de forma que $J_{k}^{\mathbb{S}}\left(\mathbf{x}^{(i+1)}\right)<J_{k}^{\mathbb{S}}\left(\mathbf{x}^{(i)}\right)$.

O critério de parada mais usual no Algoritmo 3.1 é $\left\|\nabla J_{k}^{\mathbb{S}}(\mathbf{x})^{(i)}\right\|<\epsilon$, onde $\epsilon$ é uma constante real positiva pequena. Entretanto, esse critério não leva em conta a ordem de grandeza do vetor estimado. Para contornar essa deficiência, um critério de parada mais apurado é $\left\|\nabla J_{k}^{\mathbb{S}}(\mathbf{x})^{(i)}\right\|^{2}<\rho\left\|\mathbf{x}^{(i)}\right\|^{2}$, onde $\rho$ é uma constante real positiva pequena, costumeiramente entre $10^{-4}$ e $10^{-6}$.

Devido ao fato de $J_{k}^{\mathbb{S}}\left(\mathbf{x}^{(i)}\right)$ ser função quadrática, o valor de $\Delta \mathbf{x}^{(i)}$ que corresponde à direção de descida mais íngreme (steepest descent), ou seja, 
aquela que minimiza $J_{k}^{\mathbb{S}}\left(\mathbf{x}^{(i+1)}\right)-J_{k}^{\mathbb{S}}\left(\mathbf{x}^{(i)}\right)$ quando $x^{(i+1)}=x^{(i)}+\Delta \mathbf{x}^{(i)}$, é dado pelo passo de Newton (Newton step) (28), que é definido como

$$
\Delta \mathbf{x}_{N} \triangleq-\nabla^{2}\left(J_{k}^{\mathbb{S}}(\mathbf{x})\right)^{-1} \nabla J_{k}^{\mathbb{S}}(\mathbf{x})
$$

Utilizando 3-8 e levando-se em conta que $\nabla^{2}\left(J_{k}^{\mathbb{S}}(\mathbf{x})\right)=2\left(\mathbf{A}_{k}\right)_{\mathbb{S}, \mathbb{S}}$, constata-se que $\Delta \mathbf{x}_{N}=-\mathbf{x}+\hat{\mathbf{h}}_{k}^{\mathbb{S}}$. Nesse caso, ao se escolher $\Delta \mathbf{x}=\Delta \mathbf{x}_{N}$, assegura-se que $\hat{\mathbf{h}}_{k}^{\mathbb{S}}$ é encontrado em uma única iteração. Entretanto, o cálculo de $\Delta \mathbf{x}_{N}$ também exige o cálculo da inversa de $\left(\mathbf{A}_{k}\right)_{\mathbb{S}, \mathbb{S}}$. Portanto, o processo de busca através do método de Newton não apresenta nenhuma vantagem em relação ao cálculo direto de $\hat{\mathbf{h}}_{k}^{\mathbb{S}}$ através de 3-9.

Uma alternativa ao passo de Newton para escolha de $\Delta \mathbf{x}$ é

$$
\Delta \mathbf{x}=-\mu \nabla J_{k}^{\mathbb{S}}(\mathbf{x})
$$

em que $\mu$ é uma constante real positiva. Calculando 3-6 para $\mathbf{x}^{(i)}=\mathbf{x}^{(i)}-$ $\mu^{(i)} \nabla J_{k}^{\mathbb{S}}\left(\mathbf{x}^{(i)}\right)$, derivando em relação a $\mu^{(i)}$ e igualando a zero, obtém-se o valor de $\mu^{(i)}$ que minimiza $J_{k}^{\mathbb{S}}\left(\mathbf{x}^{(i+1)}\right)-J_{k}^{\mathbb{S}}\left(\mathbf{x}^{(i)}\right)$, que é dado por

$$
\mu_{o}^{(i)}=\frac{\left\|\nabla J_{k}^{\mathbb{S}}\left(\mathbf{x}^{(i)}\right)\right\|^{2}}{2\left(\nabla J_{k}^{\mathbb{S}}\left(\mathbf{x}^{(i)}\right)\right)^{H}\left(\mathbf{A}_{k}\right)_{\mathbb{S}, \mathbb{S}} \nabla J_{k}^{\mathbb{S}}\left(\mathbf{x}^{(i)}\right)}
$$

O Algoritmo 3.2 descreve a busca da estimativa LS tendo como direção o oposto ao gradiente do custo LS e com o passo ótimo dado por 3-52.

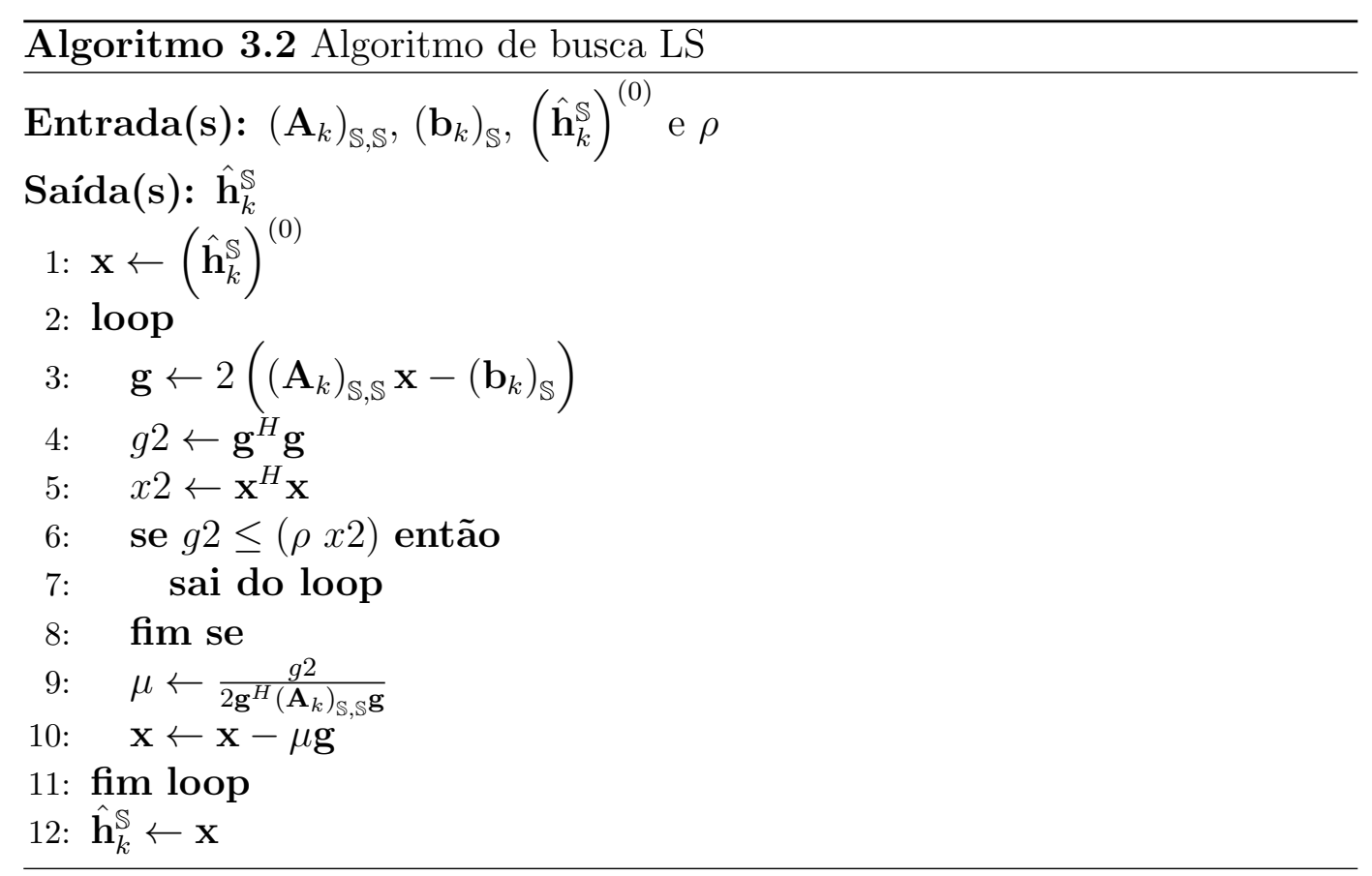




\subsection{1}

\section{Velocidade de convergência versus valor inicial da estimativa LS}

Normalmente, independentemente do algoritmo de busca, quanto mais próximo o valor inicial da estimativa em relação ao valor real, mais rápida a convergência. Observando 3-9 e considerando que $\left(\mathbf{A}_{k}\right)_{\mathbb{S}, \mathbb{S}} \stackrel{a . s .}{\longrightarrow} \mathbf{I}$, então um bom valor inicial (hot start) para o parâmetro de entrada $\left(\hat{\mathbf{h}}_{k}^{\mathbb{S}}\right)^{(0)}$ no Algoritmo 3.2 é

$$
\left(\hat{\mathbf{h}}_{k}^{\mathbb{S}}\right)^{(0)}=\left(\mathbf{b}_{k}\right)_{\mathbb{S}}
$$

No caso em que já tenha sido previamente calculado $\hat{\mathbf{h}}_{k}^{\mathbb{S}_{1}}$ e deseja-se determinar $\hat{\mathbf{h}}_{k}^{\mathbb{S}_{2}}$, sendo que $\mathbb{S}_{2} \subset \mathbb{S}_{1}$ (o que ocorre na estratégia de Encolhimentos Sucessivos, que será apresentada no próximo capítulo), um bom valor inicial $\operatorname{para}\left(\hat{\mathbf{h}}_{k}^{\mathbb{S}_{2}}\right)^{(0)}$ é

$$
\left(\hat{\mathbf{h}}_{k}^{\mathbb{S}_{2}}\right)^{(0)}=(\mathbf{I})_{\mathbb{S}_{2}, \mathbb{S}_{1}} \hat{\mathbf{h}}_{k}^{\mathbb{S}_{1}}
$$

em que $(\mathbf{I})_{\mathbb{S}_{2}, \mathbb{S}_{1}}$ é matriz formada pelas linhas da matriz-identidade $\mathbf{I}_{\mathbb{L}, \mathbb{L}}$ cujos índices pertencem a $\mathbb{S}_{2}$ e pelas colunas da matriz-identidade cujos índices pertencem a $\mathbb{S}_{1}$.

A velocidade de convergência do Algoritmo 3.2 depende do grau de condicionamento de $\left(\mathbf{A}_{k}\right)_{\mathbb{S}, \mathbb{S}}$, que é dado pela razão entre os módulos do maior e do menor autovalores da matriz. Quanto maior o grau de condicionamento, mais lenta a convergência. Admitindo que, para $k \times M$ suficientemente grande, $\left(\mathbf{A}_{k}\right)_{\mathbb{S}, \mathbb{S}} \approx \mathbf{I}$ (uma vez que $\left(\mathbf{A}_{k}\right)_{\mathbb{S}, \mathbb{S}} \stackrel{\text { a.s. }}{\longrightarrow} \mathbf{I}$ ), então o grau de condicionamento de $\left(\mathbf{A}_{k}\right)_{\mathbb{S}, \mathbb{S}}$ é pequeno e, portanto, a velocidade de convergência costuma ser rápida.

A Figura 3.4 mostra a quantidade média de iterações do Algoritmo 3.2, em função da cardinalidade de $\mathbb{S}$, para $\mathrm{N}=8, \mathrm{k}=10, \mathcal{E}_{\text {chip }} / \sigma_{z}^{2}=0 \mathrm{~dB}$ e $\rho=10^{-4}$ em $\mathrm{Q}=1000$ realizações de um sistema $\operatorname{MESS}(201,15)$. Para cada $q$-ésima realização do sistema e para cada valor de cardinalidade $c$, foi gerado um conjunto $\mathbb{S}^{(q)}$ com $\left|\mathbb{S}^{(q)}\right|=c$ de forma totalmente aleatória e independente das demais realizações. A curva (1) refere-se ao caso em que $\left(\hat{\mathbf{h}}_{k}^{\mathbb{S}_{2}}\right)^{(0)}=\mathbf{0}$. A curva (2) refere-se ao caso em que $\left(\hat{\mathbf{h}}_{k}^{\mathbb{S}_{2}}\right)^{(0)}=\left(\mathbf{b}_{k}\right)_{\mathbb{S}}$. A curva (3) refere-se ao caso em que $\left(\hat{\mathbf{h}}_{k}^{\mathbb{S}_{2}}\right)^{(0)}=\mathbf{I}_{\mathbb{S}, \mathbb{L}} \hat{\mathbf{h}}_{k}^{\mathbb{L}}$. Verifica-se que as três curvas apresentam uma quantidade média de iterações pequena. A curva (3) apresenta uma quantidade média de iterações ao menos duas vezes menor que as curvas (1) e (2), porém ao custo de se ter que calcular previamente $\hat{\mathbf{h}}_{k}^{\mathbb{L}}$. 


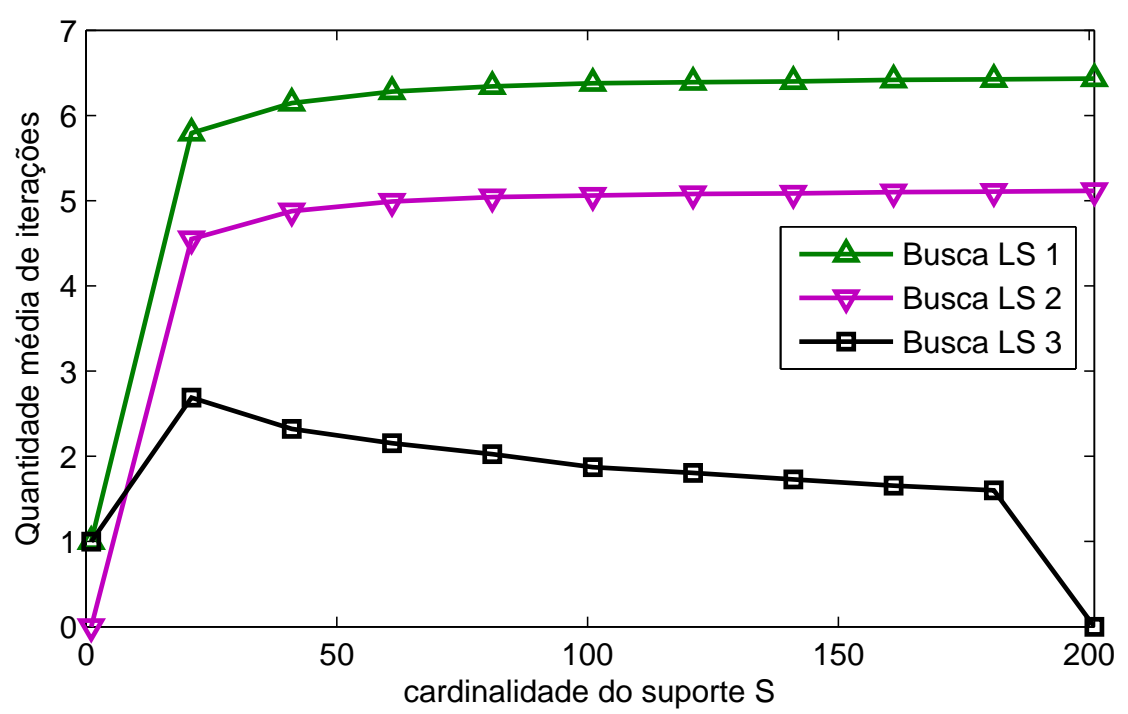

Figura 3.4: Quantidade média de iterações do Algoritmo de Busca LS em função de $|\mathbb{S}|$ para $\mathrm{N}=8, \mathrm{k}=10, \mathcal{E}_{\text {chip }} / \sigma_{z}^{2}=0 \mathrm{~dB}$ e $\rho=10^{-4}$, em $\mathrm{Q}=1000$ realizações de um sistema $\operatorname{MESS}(201,15)$, nos seguintes casos: $(1)\left(\hat{\mathbf{h}}_{k}^{\mathbb{S}}\right)^{(0)}=\mathbf{0}$, $(2)\left(\hat{\mathbf{h}}_{k}^{\mathbb{S}}\right)^{(0)}=\left(\mathbf{b}_{k}\right)_{\mathbb{S}},(3)\left(\hat{\mathbf{h}}_{k}^{\mathbb{S}}\right)^{(0)}=\mathbf{I}_{\mathbb{S}, \mathbb{L}} \hat{\mathbf{h}}_{k}^{\mathbb{L}}$

\section{8}

\section{Custo computacional}

O cálculo da estimativa LS, associada a um suporte $\mathbb{S}$ qualquer, no instante $k$, é dada por 3-9, repetida a seguir por conveniência

$$
\hat{\mathbf{h}}_{k}^{\mathbb{S}}=\left(\mathbf{A}_{k}\right)_{\mathbb{S}, \mathbb{S}}^{-1}\left(\mathbf{b}_{k}\right)_{\mathbb{S}}
$$

A estimativa LS full-support, no instante $k$, corresponde a 3-55 para o caso particular em que $\mathbb{S}=\mathbb{L}$ e pode ser calculada da seguinte forma

$$
\hat{\mathbf{h}}_{k}^{\mathbb{L}}=\mathbf{A}_{k}^{i n v} \mathbf{b}_{k}
$$

em que $\mathbf{A}_{k}^{i n v}=\mathbf{A}_{k}^{-1}$ é calculada a priori e armazenada em memória. Dessa forma, o custo computacional de 3-56, medido pelo número de operações de ponto flutuante $(F L O P S)$, corresponde ao custo computacional da operação de multiplicação algébrica entre $\mathbf{A}_{k}^{i n v}$ e $\mathbf{b}_{k}$, que é $\mathcal{O}\left(L^{2}\right)$.

Entretanto, admitindo que não seja possível pré-calcular e armazenar $\left(\mathbf{A}_{k}\right)_{\mathbb{S}, \mathbb{S}}^{-1}$ para todas as possibilidades em que $\mathbb{S} \neq \mathbb{L}$, então o custo computacional de 3-55 é predominado pelo custo computacional da inversão de $\left(\mathbf{A}_{k}\right)_{\mathbb{S}, \mathbb{S}}$, que é $\mathcal{O}\left(|\mathbb{S}|^{3}\right)$. 
Uma forma de reduzir o custo computacional de 3-55 é calcular $\hat{\mathbf{h}}_{k}^{\mathbb{S}}$ da seguinte forma

$$
\hat{\mathbf{h}}_{k}^{\mathbb{S}}=\left(\mathbf{L}_{k}^{\mathbb{S}}\right)^{H} \backslash_{T}\left(\mathbf{L}_{k}^{\mathbb{S}} \backslash_{T}\left(\mathbf{b}_{k}\right)_{\mathbb{S}}\right)
$$

em que $\mathbf{L}_{k}^{\mathbb{S}}$ é matriz triangular inferior obtida através da Decomposição de Cholesky da matrix positiva-definida $\left(\mathbf{A}_{k}\right)_{\mathbb{S}, \mathbb{S}}\left(\right.$ ou seja, $\left(\mathbf{A}_{k}\right)_{\mathbb{S}, \mathbb{S}}=\left(\mathbf{L}_{k}^{\mathbb{S}}\right)^{H} \mathbf{L}_{k}^{\mathbb{S}} \mathrm{e} \backslash_{T}$ denota divisão à esquerda por matriz triangular (29).

O custo computacional de 3-57 é a soma dos custos computacionais das duas operações $\backslash_{T}$, que é $\mathcal{O}\left(|\mathbb{S}|^{2}\right)$, e do custo computacional da Decomposição de Cholesky, que é $\mathcal{O}\left(|\mathbb{S}|^{3}\right)$.

Embora tanto o custo computacional da Decomposição de Cholesky quanto o custo computacional da inversão da matriz $\left(\mathbf{A}_{k}\right)_{\mathbb{S}, \mathbb{S}}$ seja $\mathcal{O}\left(|\mathbb{S}|^{3}\right)$, o custo computacional da Decomposição de Cholesky é cerca de três vezes menor. Portanto, para $\mathbb{S}$ com cardinalidade muito grande, o cálculo de $\hat{\mathbf{h}}_{k}^{\mathbb{S}}$ através de 3-57 exige cerca de 1/3 da quantidade de flops quando comparado ao cálculo através do emprego direto de 3-55.

A Figura 3.5 mostra o tempo médio gasto para determinar a estimativa LS $\hat{\mathbf{h}}_{k}^{\mathbb{S}}$ em função de $|\mathbb{S}|$, para $\mathrm{N}=8, \mathrm{k}=10, \mathcal{E}_{\text {chip }} / \sigma_{z}^{2}=0 \mathrm{~dB}, \rho=10^{-4}$, em $\mathrm{Q}=1000$ realizações de um sistema $\operatorname{MESS}(201,15)$, empregando os seguinte métodos:

1. cálculo direto através de 3-55;

2. cálculo através de 3-57, empregando-se a Decomposição de Cholesky de $\left(\mathbf{A}_{k}\right)_{\mathbb{S}, \mathbb{S}}$

3. busca através do Algoritmo 3.2, com $\left(\hat{\mathbf{h}}_{k}^{\mathbb{S}}\right)^{(0)}=\mathbf{0}$;

4. busca através do Algoritmo 3.2, com $\left(\hat{\mathbf{h}}_{k}^{\mathbb{S}}\right)^{(0)}=\left(\mathbf{b}_{k}\right)_{\mathbb{S}}$;

5. busca através do Algoritmo 3.2, com $\left(\hat{\mathbf{h}}_{k}^{\mathbb{S}}\right)^{(0)}=\mathbf{I}_{\mathbb{S}, \mathbb{L}} \hat{\mathbf{h}}_{k}^{\mathbb{L}}$, calculandose previamente $\hat{\mathbf{h}}_{k}^{\mathbb{L}}$ através de $3-56$ (com $\mathbf{A}_{k}^{\text {inv }}$ calculada a priori e armazenada em memória).

Os tempos mostrados na Figura 3.5 foram obtidos com os métodos implementados em Matlab em um computador com Processador Intel i5M460@2,53GHz com 4GB de memória RAM. Nessa figura, é possível constatar que:

- apesar de, teoricamente a quantidade de flops da Decomposição de Cholesky ser cerca de $1 / 3$ a quantidade de flops da operação de inversão de 


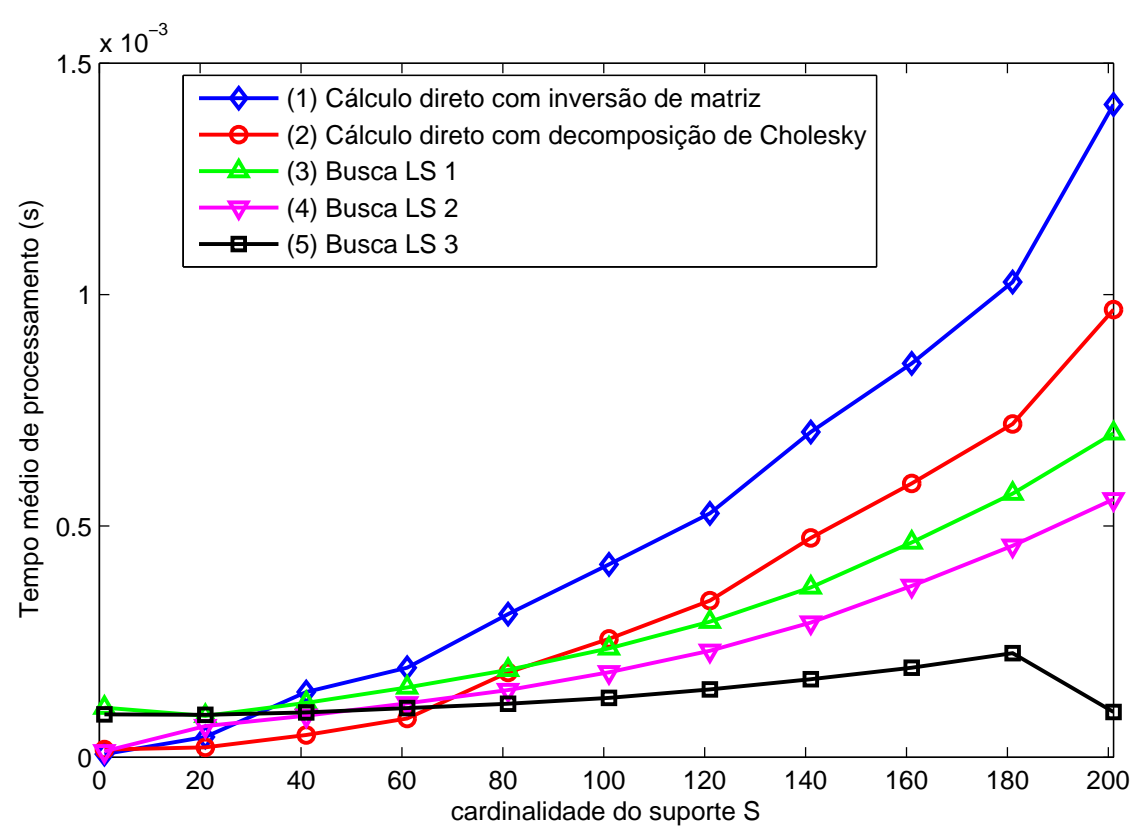

Figura 3.5: Tempo médio gasto para determinar a estimativa LS $\hat{\mathbf{h}}_{k}^{\mathbb{S}}$ em função de $|\mathbb{S}|$, para $\mathrm{N}=8, \mathrm{k}=10, \mathcal{E}_{\text {chip }} / \sigma_{z}^{2}=0 \mathrm{~dB}, \rho=10^{-4}$, em $\mathrm{Q}=1000$ realizações de um sistema MESS(201,15), empregando os seguinte métodos: (1) cálculo direto através de 3-55; (2) cálculo através de 3-57, empregando-se a Decomposição de Cholesky de $\left(\mathbf{A}_{k}\right)_{\mathbb{S}, \mathbb{S}} ;$ (3) busca através do Algoritmo 3.2, $\operatorname{com}\left(\hat{\mathbf{h}}_{k}^{\mathbb{S}}\right)^{(0)}=\mathbf{0}$; (4) busca através do Algoritmo 3.2, com $\left(\hat{\mathbf{h}}_{k}^{\mathbb{S}}\right)^{(0)}=\left(\mathbf{b}_{k}\right)_{\mathbb{S}} ;(5)$ busca através do Algoritmo 3.2, com $\left(\hat{\mathbf{h}}_{k}^{\mathbb{S}}\right)^{(0)}=\mathbf{I}_{\mathbb{S}, \mathbb{L}} \hat{\mathbf{h}}_{k}^{\mathbb{L}}$, calculando-se previamente $\hat{\mathbf{h}}_{k}^{\mathbb{L}}$ através de 3-56 (com $\mathbf{A}_{k}^{i n v}$ calculada previamente e armazenada em memória).

matrizes, na prática (ao menos nas simulações realizadas), o desempenho do método (2) de fato é bem melhor que do método (1), mas não chega a ser três vezes melhor;

- para $|\mathbb{S}|$ relativamente baixo $(|\mathbb{S}| \leq 60$ aproximadamente), o método (2) é o mais rápido para a determinação da estimativa LS;

- para $|\mathbb{S}|$ alto $(|\mathbb{S}|>60)$, o método (5) é o mais rápido para a determinação da estimativa LS.

\section{9}

\section{Considerações finais}

Neste capítulo, foi apresentada a técnica de estimação LS com suporte reduzido, em que a técnica de estimação LS full-support ordinária pode ser vista como um caso particular da estimação LS com suporte reduzido. Nesse sentido, o presente capítulo se propôs a estender todo o arcabouço teórico, tais 
como análises de convergência das estimativas e do custo LS tradicionalmente apresentadas para o caso full-support, para o modelo com suporte reduzido.

Algumas conclusões apresentadas nesse capítulo embasam as técnicas de estimação de sistemas esparsos originalmente elaboradas e apresentadas no próximo capítulo. Dentre elas, convém destacar:

1. De acordo com a equação 3-31, a estimativa LS referente a um suporte $\mathbb{S}$ converge com probabilidade 1 para o vetor formado pelos elementos da resposta ao impulso verdadeira do sistema cujos índices pertencem a $\mathbb{S}$. Portanto, se $\mathbb{S}$ contém o suporte verdadeiro do sistema, então a estimativa LS com suporte reduzido converge para a resposta ao impulso verdadeira do sistema;

2. De acordo com a equação 3-36, o custo LS referente ao suporte $\mathbb{S}$ converge com probabilidade 1 para o patamar de ruído-mais-interferência se $\mathbb{S}$ contém o suporte verdadeiro do sistema. Por outro lado, de acordo com a equação $3-44$, o custo LS referente ao suporte $\mathbb{S}$ converge com probabilidade 1 para o valor correspondente ao patamar do ruído-maisinterferência multiplicado por um fator diretamente proporcional à SINR e à razão entre a energia do vetor formado pelos elementos da resposta ao impulso verdadeira do sistema cujos índices não pertencem a $\mathbb{S}$ e a energia total da resposta ao impulso verdadeira do sistema. As equações 3-36 e 3-44, juntas, servirão como mecanismo para avaliar se a estimação do suporte do sistema é boa ou não;

3. o Teorema 3.1 demonstra que toda sequência $\left\{J_{k}\left(\mathbb{S}_{i}\right) \mid \mathbb{S}_{i+1} \subset \mathbb{S}_{i}\right\}$ é monótona não-decrescente e servirá de base para a estratégia de Encolhimentos Sucessivos, apresentada no próximo capítulo;

4. a Estimação LS com suporte reduzido também pode ser processada por blocos, mantendo-se as mesmas propriedades de convergência do custo e da estimativa LS;

5. a análise do custo computacional para determinação das estimativas LS com suporte reduzido mostram que, se a cardinalidade do suporte for pequena (abaixo de sessenta aproximadamente), o cálculo da estimativa com o emprego da Decomposição de Cholesky é o método mais eficiente; por outro lado, se a cardinalidade do suporte for grande (acima de sessenta aproximadamente), a determinação da estimativa LS através do algoritmo de busca pelo gradiente, com adequada iniciação do algoritmo, é a mais eficiente. 


\section{Métodos propostos para estimação de sistemas esparsos}

\section{1}

\section{Introdução}

Uma técnica amplamente empregada para a identificação de sistemas lineares e invariantes no tempo é a estimação LS full-support ordinária. A estimação LS full-support ordinária é uma técnica de baixa complexidade computacional que produz estimativas consistentes e despolarizadas. Entretanto, como foi visto no capítulo anterior, uma deficiência desta técnica de estimação é apresentar baixa velocidade de convergência quando aplicada à estimação de sistemas esparsos em que a resposta ao impulso em tempo discreto é representada por um vetor de comprimento longo.

Visando acelerar a velocidade de convergência da estimação LS de sistemas esparsos, quatro novos métodos são originalmente elaborados e apresentados neste capítulo. São eles:

1. Encolhimentos Sucessivos;

2. Expansões Sucessivas;

3. Minimização da Norma $l_{1}$;

4. Regularização Automática do Custo LS.

Dos quatro métodos citados, os três primeiros se baseiam na técnica de estimação LS com suporte reduzido, o que envolve a escolha do suporte da estimativa LS da resposta ao impulso do sistema de forma adequada.

Os quatro métodos propostos compartilham o mesmo parâmetro de funcionamento $J_{\max }$, que corresponde ao máximo custo LS admitido. Em todos os métodos propostos, quanto maior $J_{\max }$, mais esparsa a estimativa produzida. Por isso, precedendo a apresentação dos métodos propostos, na seção seguinte é apresentado um critério para a escolha do valor de $J_{\max }$. Visando explicar porque a norma $l_{1}$ costuma ser empregada heuristicamente como medida de esparsidade, na seção subsequente é mostrado, como uma abordagem inédita, o vínculo existente entre a norma $l_{1}$ e a pseudo-norma $l_{0}$. Ainda precedendo a apresentação dos métodos propostos para estimação de sistemas esparsos, o problema de estimação LS ótima de sistemas esparsos é caracterizado. Finalmente, os métodos para estimação de sistemas esparsos originalmente elaborados nesta Tese são apresentados. 


\section{2}

\section{O valor máximo admitido para o custo LS}

A estimação LS full-support ordinária visa minimizar o custo LS. Por definição, a estimativa LS é aquela que minimiza o custo LS. Como o custo LS é função convexa, então a estimativa LS é única. Portanto, a partir do momento em que qualquer outro método de estimação produz estimativas diferentes da estimativa LS, é admitido um relaxamento do custo LS, no sentido de que as estimativas produzidas, como não coincidem com a estimativa LS, não minimizam o custo LS.

Os métodos de estimação de sistemas esparsos propostos neste capítulo compartilham o parâmetro $J_{\max }$, que corresponde ao máximo custo LS admitido. Ou seja, as estimativas esparsas produzidas pelos métodos propostos apresentam custo LS menor ou igual a $J_{\max }$.

A equação 3-36 mostra que $J_{k}(\mathbb{L})$ converge com probabilidade 1 para o patamar de ruído-mais-interferência $M \sigma_{z}^{2}$ quando $k$ tende a infinito. Por isso, a escolha de $J_{\text {max }}$ pode ser vinculada a $M \sigma_{z}^{2}$, da seguinte forma

$$
J_{\max }=\lambda M \sigma_{z}^{2} \quad \lambda>1
$$

onde $\lambda$ é um parâmetro real maior que 1 , denominado fator de relaxamento do custo LS. Entretanto, a escolha indireta do valor de $J_{\max }$ através da escolha do valor de $\lambda$ exige a estimação do patamar de ruído-mais-interferência $M \sigma_{z}^{2}$. Um procedimento semelhante é escolher $J_{\max }$ em função de $J_{k}(\mathbb{L})$, ou seja,

$$
J_{\max }=\lambda J_{k}(\mathbb{L}) \quad \lambda>1
$$

onde $J_{k}(\mathbb{L})$ pode ser calculado a partir de 3-11 ou de 3-12, resultando em

$$
J_{k}(\mathbb{L})=R_{k}-\mathbf{b}_{k}^{H}\left(\mathbf{A}_{k}\right)^{-1} \mathbf{b}_{k}=R_{k}-\mathbf{b}_{k}^{H} \hat{\mathbf{h}}_{k}^{\mathbb{L}}
$$

Considerando que $\mathbf{A}_{k} \stackrel{\text { a.s. }}{\longrightarrow} \mathbf{I}$, para $k$ suficientemente grande, o valor de $J_{k}(\mathbb{L})$ em 4-3 pode ser aproximado como

$$
J_{k}(\mathbb{L}) \approx R_{k}-\left\|\mathbf{b}_{k}\right\|^{2}
$$

Este procedimento para a obtenção de $J_{\max }$ dispensa a estimação de $\sigma_{z}^{2}$ e, por isso, é o procedimento adotado ao longo deste trabalho. Além disso, conforme mostra 3-36, como $J_{k}(\mathbb{L})$ tende com probabilidade 1 a $M \sigma_{z}^{2}$, então para qualquer valor de $\lambda$, o valor de $J_{\max }$ obtido através de 4-2 converge com probabilidade 1 para o valor obtido através de 4-1. 


\subsection{1}

\section{Critério para escolha do fator de relaxamento do custo LS}

Como o valor de $J_{\max }$ é obtido indiretamente, a partir do valor do fator de relaxamento $\lambda$, torna-se necessário o estabelecimento de algum critério para a escolha do valor de $\lambda$.

Dos quatro métodos de estimação de sistemas esparsos propostos nesta Tese, os métodos de Encolhimentos Sucessivos, Expansões Sucessivas e Minimização da Norma $l_{1}$ provocam um encolhimento do suporte, no sentido em que o suporte $\hat{\mathbb{S}}$ da estimativa $\hat{\mathbf{h}}$ é um subconjunto do suporte $\mathbb{L}$.

Ao longo deste trabalho, os encolhimentos são classificados em quatro eventos distintos: quando $\hat{\mathbb{S}}=\mathbb{S}_{\mathbf{h}}$, diz-se que ocorreu um encolhimento correto; quando $\hat{\mathbb{S}} \supset \mathbb{S}_{\mathbf{h}}$, diz-se que ocorreu um sub-encolhimento ou um encolhimento pequeno; quando $\hat{\mathbb{S}} \subset \mathbb{S}_{\mathbf{h}}$, diz-se que ocorreu um super-encolhimento ou um encolhimento excessivo e; quando $\hat{\mathbb{S}}$ não corresponde a nenhum dos eventos anteriores, diz-se que ocorreu um encolhimento disforme.

O enquadramento de $\hat{\mathbb{S}}$ nesses quatro eventos é utilizado neste trabalho apenas para fins de análise de desempenho dos métodos propostos e só faz sentido se $\mathbb{S}_{\mathbf{h}} \subset \mathbb{L}$, ou seja, se $\mathbf{h}$ contém elementos nulos. Nesse caso, o Teorema 3.1 mostra que, na ocorrência de sub-encolhimento, a distância entre a verdadeira resposta ao impulso do sistema e sua respectiva estimativa LS associada a $\hat{\mathbb{S}}$ é menor que a distância entre a verdadeira resposta ao impulso do sistema e sua respectiva estimativa LS associada a $\mathbb{L}$, porém maior que a distância entre a verdadeira resposta ao impulso do sistema e sua respectiva estimativa LS associada ao suporte verdadeiro do sistema $\mathbb{S}_{\mathbf{h}}$. Por outro lado, na ocorrência de um superencolhimento ou um encolhimento disforme, a equação 3-44 mostra que, em média, a distância entre a verdadeira resposta ao impulso do sistema e sua respectiva estimativa LS associada a $\hat{\mathbb{S}}$ cresce significativamente.

A equação 3-44 mostra que, quanto maior o valor de $k$, à medida em que são geradas sucessivas estimativas $\left\{\hat{\mathbf{h}}_{k}^{\mathbb{S}_{i}} \mid \mathbb{S}_{i} \supset \mathbb{S}_{i+1}, i=1,2, \ldots\right\}$, então mais provavelmente

$$
J_{k}^{\mathbb{S}}\left(\hat{\mathbf{h}}_{k}^{\mathbb{S}}\right) \approx\left[1+\frac{\mathrm{SINR}}{M} \frac{\left\|(\mathbf{h})_{\tilde{\mathbb{S}}}\right\|^{2}}{\|\mathbf{h}\|^{2}}\right] M \sigma_{z}^{2}
$$

Portanto, ao longo de encolhimentos sucessivos, embora a sequência dos custos LS $\left\{J_{k}\left(\mathbb{S}_{i}\right) \mid \mathbb{S}_{i+1} \subset \mathbb{S}_{i}\right\}$ cresça, conforme ficou demonstrado na seção 3.6, os valores de $J_{k}\left(\mathbb{S}_{i}\right)$ tendem a permanecer muito próximos de $J_{k}(\mathbb{L})$ enquanto $\mathbb{S}_{i} \supseteq \mathbb{S}_{\mathbf{h}}$. Por outro lado, o valor de $J_{k}\left(\mathbb{S}_{i}\right)$ tende a ser significativamente maior que $J_{k}(\mathbb{L})$ se $\mathbb{S}_{i} \nsubseteq \mathbb{S}_{\mathbf{h}}$. 
À medida em que $k$ aumenta, os métodos de estimação que provocam um encolhimento do suporte tendem a aumentar a robustez contra encolhimentos excessivos provocados pelo ruído (mais interferência). Para exemplificar, considere o caso fictício em que $\operatorname{SINR}=30 \mathrm{~dB}, M=100, \sigma_{z}^{2}=0,01 \mathrm{e}$ $\left|\mathbb{S}_{\mathbf{h}}\right|=10$, sendo o valor absoluto de cada um dos elementos não-nulos de $\mathbf{h}$ aproximadamente o mesmo. Neste exemplo, para $k$ suficientemente grande, a equação 3-36 mostra que, enquanto $\mathbb{S}_{i} \supseteq \mathbb{S}_{\mathbf{h}}$, tem-se que, muito provavelmente, $J_{k}\left(\mathbb{S}_{i}\right) \approx 1$. Por outro lado, a equação 3-44 mostra que. se apenas um dos índices dos elementos não-nulos de $\mathbf{h}$ não pertence a $\mathbb{S}_{i}$, então muito provavelmente $J_{k}\left(\mathbb{S}_{i}\right) \approx 2$. Este salto brusco no valor de $J_{k}(\mathbb{S})$ nitidamente indica um encolhimento excessivo.

Apesar da robustez contra encolhimentos excessivos provocados pelo ruído, não convém atribuir desnecessariamente um valor grande para $\lambda$, pois isso pode eventualmente deixar o método de estimação mais susceptível a encolhimentos excessivos provocados pela natureza estocástica do sistema e, portanto, mais instável.

Diante do exposto, apesar da intrínseca robustez contra encolhimentos excessivos provocados pelo ruído, conclui-se que $\lambda$ deve ser grande o suficiente para permitir que os métodos de estimação propostos produzam estimativas com adequado encolhimento do suporte (idealmente sem sub-encolhimentos e somente encolhimentos corretos), ao mesmo tempo em que não pode ser grande demais, para que esses métodos não fiquem demasiadamente susceptíveis, a cada nova realização do sistema, a encolhimentos excessivos provocados pela natureza estocástica do sistema.

Ao longo deste trabalho, a escolha de $\lambda$ foi feita de maneira empírica, tendo como objetivo acelerar a velocidade de convergência.

\section{3}

\section{Norma L1 como medida de esparsidade}

Para que seja possível a implementação de técnicas de estimação de sistemas esparsos, é necessário medir a esparsidade das estimativas produzidas através de alguma função apropriada. Uma forma de medir a esparsidade de um vetor $\mathbf{x}$ qualquer é através da sua pseudo-norma $l_{0}$, denotada por $\|\mathbf{x}\|_{0}$. Porém, a função $f(\mathbf{x})=\|\mathbf{x}\|_{0}$ é não-linear e não-diferenciável, e a estimação baseada na pseudo-norma $l_{0}$ é um problema NP-hard.

Algumas técnicas de estimação utilizam como métrica de esparsidade uma aproximação convexa e diferenciável de $\|\mathbf{x}\|_{0}$. Outras técnicas utilizam heuris- 
ticamente $\|\mathbf{x}\|_{1}$ (a norma $l_{1}$ ) como medida de esparsidade de $\mathbf{x}$, haja vista se tratar de uma função convexa e diferenciável, o que permite um tratamento analítico ao problema de estimação.

A fim de analisar a conexão existente entre a pseudo-norma $l_{0}$ e a norma $l_{1}$, considere a função $L_{0}(\mathbf{x})$, definida como segue

$$
L_{0}(\mathbf{x})=\left\{\begin{array}{cl}
\left(\frac{\|\mathbf{x}\|_{1}}{\|\mathbf{x}\|_{2}}\right)^{2} & \mathbf{x} \neq \mathbf{0} \\
0 & \mathbf{x}=\mathbf{0}
\end{array}\right.
$$

É possível verificar três interessantes propriedades de $L_{0}(\mathbf{x})$, enunciados pelos três teoremas a seguir ${ }^{1}$ :

Teorema 4.1 Se todos os $S$ elementos não-nulos de $\mathbf{x}$ tiverem o mesmo módulo, então $L_{0}(\mathbf{x})=S$.

Prova. A prova decorre trivialmente de $4-6$.

Teorema 4.2 Se $\alpha \in \mathbb{C}$ e $\alpha \neq 0$, então $L_{0}(\alpha \mathbf{x})=L_{0}(\mathbf{x})$.

Prova. A prova decorre trivialmente de 4-6.

Teorema 4.3 Se $\mathbf{x}$ for um vetor não-nulo de comprimento $N$, então $L_{0}(\mathbf{x}) \leq$ $N$, com a igualdade atingida se e somente se todos os elementos de $\mathbf{x}$ tiverem o mesmo módulo.

Prova. Considere o problema de encontrar o ponto de máximo (ou de mínimo) de $L_{0}(\mathbf{x})$ condicionado a $\|\mathbf{x}\|_{2}^{2}=1$. Como, neste caso, $L_{0}(\mathbf{x})=\|\mathbf{x}\|_{1}^{2}$, o problema é equivalente a minimizar $l_{1}(\mathbf{x})=\|\mathbf{x}\|_{1}$, condicionado a $\|\mathbf{x}\|_{2}^{2}=1$. O Lagrangiano de $l_{1}(\mathbf{x})$ é dado por

$$
\mathfrak{L}(\mathbf{x}, \lambda)=\sum_{i=1}^{N}\left|x_{i}\right|+\lambda\left(\sum_{i=1}^{N}\left|x_{i}\right|^{2}-1\right)
$$

Portanto, $\mathfrak{L}(\mathbf{x}, \lambda)$ equivale à função

$$
\mathfrak{L}(\mathbf{m}, \lambda)=\sum_{i=1}^{N} m_{i}+\lambda\left(\sum_{i=1}^{N} m_{i}^{2}-1\right)
$$

onde $\mathbf{m}=\left[\begin{array}{lll}m_{1} & m_{2} \ldots m_{N}\end{array}\right]^{T}$ e $m_{i}=\left|x_{i}\right|, i=1,2, \ldots, N$.

Calculando a derivada parcial de $\mathfrak{L}(\mathbf{m}, \lambda)$ em relação a $m_{i}$ e igualando a zero, tem-se que

${ }^{1} \mathrm{~A}$ função $L_{0}(\mathbf{x})$ e algumas de suas propriedades são mostradas, com abordagem semelhante, em (30). 


$$
\frac{\partial}{\partial m_{i}} \mathfrak{L}(\mathbf{m}, \lambda)=1+2 \lambda m_{i}=0 \forall i
$$

Logo, $m_{i}=-1 / 2 \lambda \forall i$. Como $m_{i}>0$ (a condição estritamente maior decorre do fato que $\left.\|\mathbf{x}\|_{2}=1\right)$, então tem-se que $\lambda<0$ e, com isso, $\mathfrak{L}(\mathbf{m}, \lambda)$ é um parabolóide côncavo em $\mathbf{m}$. Portanto, $\mathbf{m}=(-1 / 2 \lambda) \mathbf{1}$ corresponde ao ponto de máximo de $L_{0}(\mathbf{x})$, condicionado a $\|\mathbf{x}\|_{2}=1$.

Utilizando o Teorema 4.1, conclui-se que o valor máximo de $L_{0}(\mathbf{x})$ é $N$, condicionado a $\|\mathbf{x}\|_{2}=1$. Utilizando o Teorema 4.2, conclui-se finalmente que, sem restrição de dominio, quando todos os $N$ elementos de $\mathbf{x}$ têm o mesmo módulo, então a função $L_{0}(\mathbf{x})$ atinge seu valor máximo $\max L_{0}(\mathbf{x})=N$.

As três propriedades apresentadas fazem de $L_{0}(\mathbf{x})$ uma boa heurística para medir a esparsidade de $\mathbf{x}$. Para ilustrar, se um vetor $\mathbf{x}$, de comprimento $N$, tiver apenas $S$ elementos significativos (por exemplo, tendendo a infinito) e os outros $N-S$ elementos desprezíveis (por exemplo, tendendo a zero), verifica-se que $L_{0}(\mathbf{x}) \approx S$. Portanto, $L_{0}(\mathbf{x})$ pode ser vista como uma espécie de aproximação contínua e diferenciável de $\|\mathbf{x}\|_{0}$, ou seja,

$$
L_{0}(\mathbf{x})=\|\overline{\mathbf{x}}\|_{1}^{2} \approx\|\mathbf{x}\|_{0} \quad \mathbf{x} \neq \mathbf{0}
$$

Ou seja, dado um vetor $\overline{\mathbf{x}}=\mathbf{x} /\|\mathbf{x}\|_{2}$ (ou seja, $\|\overline{\mathbf{x}}\|_{2}=1$ ), conclui-se que $\|\overline{\mathbf{x}}\|_{1}^{2}$ pode ser interpretada como uma aproximação de $\|\mathbf{x}\|_{0}$ (ou equivalentemente, de $\|\overline{\mathbf{x}}\|_{0}$. Além disso, a norma $l_{1}$ tem as vantagens de ser contínua, diferenciável (exceto nos vetores que contêm elementos iguais a zero) e convexa. Por essas razões, propõe-se nesta Tese a função $L_{0}(\mathbf{x})$ como uma heurística para medir a esparsidade de um vetor $\mathbf{x}$ qualquer.

A Figura 4.1 mostra a faixa delimitada por um desvio-padrão acima e abaixo da média da função $L_{0}(\mathbf{h})$ em função da cardinalidade de $\mathbf{h}$, estimada a partir de $Q=1000$ realizações independentes de um sistema $\operatorname{MESS}(100, S)$, para cada valor de $S=1,2, \ldots, 100$. Para sistemas MESS em particular, verifica-se que a média da função $L_{0}(\mathbf{h})$ varia linearmente com $\|\mathbf{h}\|_{0}=S$. Mais precisamente, verifica-se que a média de $L_{0}(\mathbf{h})$ é cerca de 20 por cento menor que $\|\mathbf{h}\|_{0}$, para todos os valores de $\|\mathbf{h}\|_{0}$. Além disso, nota-se que o desvio-padrão da função é muito pequeno. Portanto, $1,2 \times L_{0}(\mathbf{h})$ corresponde a uma boa estimativa da cardinalidade do suporte de uma realização $\mathbf{h}$ de um sistema MESS. 


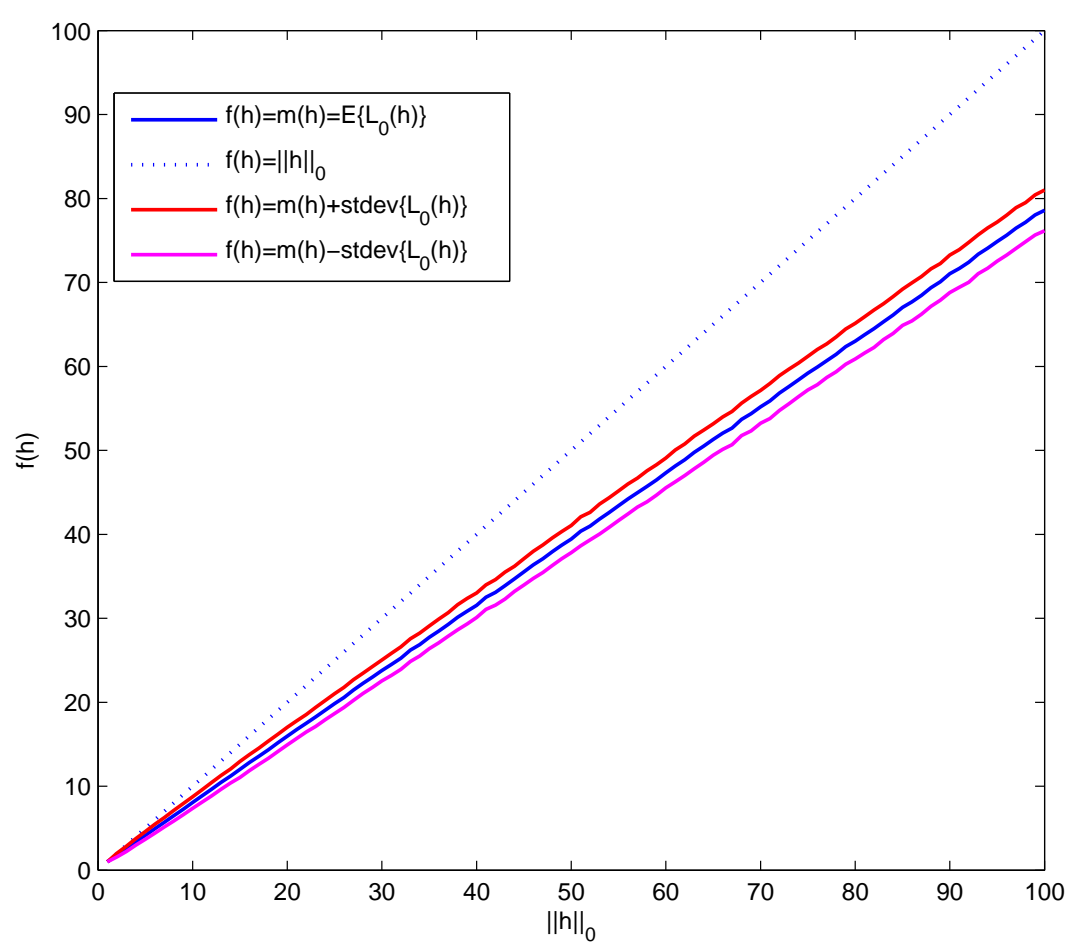

Figura 4.1: Variação da média e do desvio-padrão de $L_{0}(\mathbf{h})$ em função de $S$, estimadas em $Q=1000$ realizações independentes de um sistema $\operatorname{MESS}(100, \mathrm{~S})$, para cada valor de $S=1,2, \ldots, 100$

\section{4}

\section{Estimação LS ótima}

Como foi visto no capítulo anterior, uma alternativa à técnica de estimação LS full-support ordinária é a estimação LS com suporte reduzido. Como ambas as técnicas de estimação produzem estimativas consistentes e despolarizadas, então a estimação LS de sistemas esparsos pode ser enquadrada como um problema de estimação do suporte $\mathbb{S}_{\mathbf{h}}$ da resposta ao impulso do sistema, seguida do cálculo da estimativa LS associado ao suporte estimado $\hat{\mathbb{S}}$.

Seja $\mathbb{H}(\mathbb{L})$ o conjunto formado por todos os possíveis subconjuntos de $\mathbb{L}$, isto é,

$$
\mathbb{H}(\mathbb{L})=\{\mathbb{S} \mid \mathbb{S} \subseteq \mathbb{L}\}
$$

e $\mathbb{H}\left(\mathbb{L}, J_{\max }\right)$ o subconjunto de $\mathbb{H}(\mathbb{L})$ dado por

$$
\mathbb{H}\left(\mathbb{L}, J_{\max }\right)=\left\{\mathbb{S} \mid \mathbb{S} \in \mathbb{H}(\mathbb{L}) \text { e } J_{k}(\mathbb{S})<J_{\max }\right\}
$$


onde $J_{\max }$ é o máximo custo LS admitido.

Seja $\mathbb{H}_{\min }\left(\mathbb{L}, J_{\max }\right)$ o conjunto dos elementos de $\mathbb{H}\left(\mathbb{L}, J_{\max }\right)$ com mínima cardinalidade, isto é,

$$
\mathbb{H}_{\min }\left(\mathbb{L}, J_{\max }\right)=\left\{\mathbb{S}_{i} \mid \mathbb{S}_{i} \in \mathbb{H}\left(\mathbb{L}, J_{\max }\right) \text { e }\left|\mathbb{S}_{i}\right| \leq\left|\mathbb{S}_{j}\right| \quad \forall j \neq i\right\}
$$

Dado $J_{\max }$, a estimação LS ótima de sistemas esparsos é definida nesta Tese como

$$
\hat{\mathbf{h}}_{k}^{\mathbb{S}^{*}}=\arg \min _{\mathbf{x}} J_{k}^{\mathbb{S}^{*}}(\mathbf{x})
$$

tal que

$$
\mathbb{S}^{*}=\arg \min _{\mathbb{S}} J_{k}(\mathbb{S}) \quad \mathbb{S} \in \mathbb{H}_{\min }\left(\mathbb{L}, J_{\max }\right)
$$

Em suma, este critério estabelece como estimativa ótima o vetor $\hat{\mathbf{h}}_{k}^{\mathbb{S}^{*}}$, associado ao suporte $\mathbb{S}^{*}$, de mínima cardinalidade, que apresenta o menor valor $J_{k}(\mathbb{S})$ dentre os subconjuntos $\mathbb{S}$ de $\mathbb{L}$, tal que $J_{k}(\mathbb{S})<J_{\max }$.

A busca pela solução $\hat{\mathbf{h}}_{k}^{\mathbb{S}^{*}}$ ao problema de estimação LS ótima de sistemas esparsos pode envolver o cálculo de $J_{k}(\mathbb{S})$ para até $2^{|\mathbb{L}|}-1$ possibilidades de $\mathbb{S}$ (o que corresponde à quantidade de todos os possíveis subconjuntos de $\mathbb{L}$, exceto o conjunto-vazio $\emptyset$ ), o que pode ser computacionalmente inviável quando a cardinalidade de $\mathbb{L}$ é grande. Por isso, esta Tese apresenta, nas seções seguintes, quatro novos métodos sub-ótimos de estimação LS de sistemas esparsos, que são: Encolhimentos Sucessivos, Expansões Sucessivas, Minimização da Norma $l_{1}$ e Regularização Automática do Custo LS.

\section{5}

\section{Método de Encolhimentos Sucessivos}

\subsection{1}

\section{Introdução}

O Método de Encolhimentos Sucessivos ${ }^{2}$, proposto nesta seção, constitui-se num método de estimação LS sub-ótimo. Este método constrói, começando pela estimativa LS full-support (ou seja, começando com $\mathbb{S}^{(1)}=\mathbb{L}$ ), uma sequência de estimativas LS $\left\{\hat{\mathbf{h}}_{k}^{\mathbb{S}(i)}, i=1,2, \ldots\right\}$ tais que $\mathbb{S}^{(i+1)} \subset \mathbb{S}^{(i)}$, enquanto $J\left(\mathbb{S}^{(i)}\right)<J_{\max }$

\footnotetext{
${ }^{2}$ Uma descrição resumida deste método foi publicada em (31).
} 
Cada $i$-ésima iteração do Método de Encolhimentos Sucessivos pode ser estruturado em três estapas, que são:

- Decimação Iterativa - consiste em zerar sucessivamente os elementos não-nulos da estimativa $\mathbf{x}^{(i)}$, associada ao suporte $\mathbb{S}^{(i)}$, começando pelo elemento de menor módulo e prosseguindo em direção ao elemento de maior módulo, resultando em uma nova estimativa $\mathbf{x}^{(i+1)}$, com suporte $\mathbb{S}^{(i+1)}$, onde $\mathbb{S}^{(i+1)} \subset \mathbb{S}^{(i)}$

- Minimização Linear - consiste no cálculo da estimativa que apresenta o menor custo LS, restrita à reta varrida pelo vetor obtido na etapa anterior de decimação;

- Despolarização - consiste no cálculo da estimativa LS $\hat{\mathbf{h}}_{k}^{\mathbb{S}^{(i)}}$, associada ao suporte $\mathbb{S}^{(i)}$, obtido na etapa de decimação.

\subsection{2}

\section{Decimação iterativa}

A técnica de Decimação Iterativa, proposta nesta subseção, consiste em zerar sucessivamente os elementos não-nulos de $\mathbf{x}$, começando pelo elemento de menor módulo e prosseguindo em direção ao elemento de maior módulo, construindo assim um novo vetor $\mathbf{y}$, enquanto a condição $J_{k}(\mathbf{y})<J_{\max }$ permanece satisfeita. A técnica de Decimação Iterativa, descrita no Algoritmo 4.1, pode ser enquadrada como uma técnica de soft-thresholding e difere-se da técnica de hard-thresholding em (11) pelo fato de que a condição $J_{k}(\mathbf{y})<J_{\max }$ é verificada de forma simultânea à construção de $\mathbf{y}$.

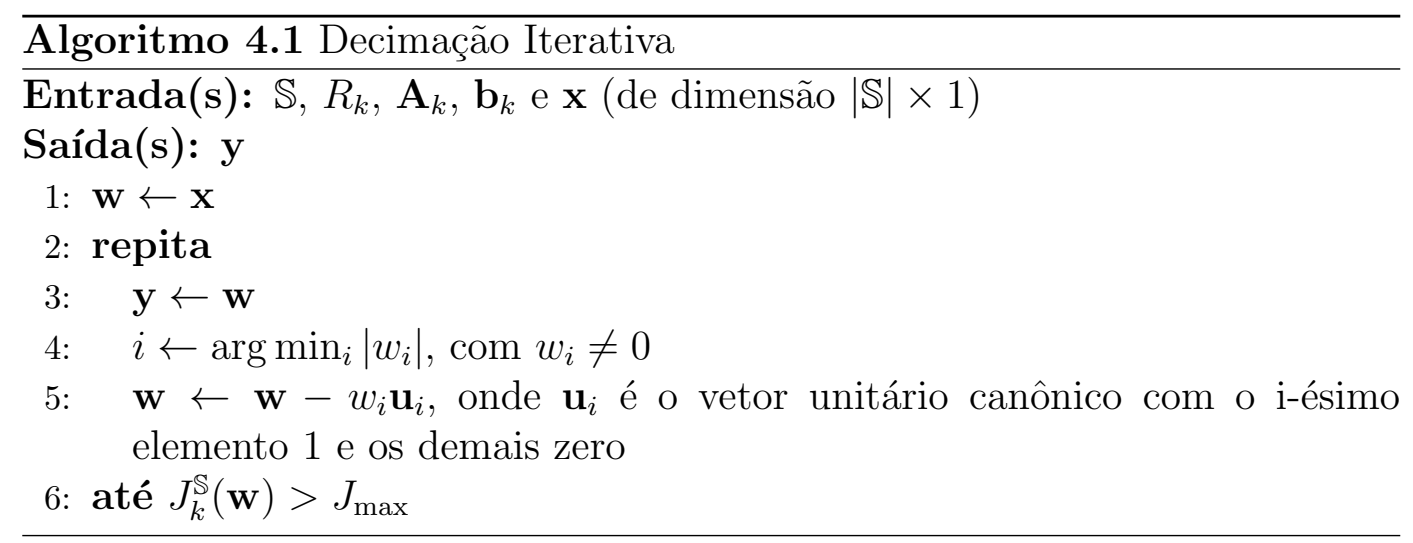

Aplicando 3-6 para $\mathbf{y}=\mathbf{x}+\Delta \mathbf{x}$, obtém-se a seguinte expressão para o cálculo de $J_{k}^{\mathbb{S}}(\mathbf{y})$ em função de $J_{k}^{\mathbb{S}}(\mathbf{x})$.

$$
J_{k}^{\mathbb{S}}(\mathbf{y})=J_{k}^{\mathbb{S}}(\mathbf{x})+\Re\left\{\Delta \mathbf{x}^{H} \nabla J_{k}^{\mathbb{S}}(\mathbf{x})\right\}+\Delta \mathbf{x}^{H}\left(\mathbf{A}_{k}\right)_{\mathbb{S}, \mathbb{S}} \Delta \mathbf{x}
$$


onde $\nabla J_{k}^{\mathbb{S}}(\mathbf{x})$ é obtido a partir de 3-8. Aplicando 3-8 para $\mathbf{y}=\mathbf{x}+\Delta \mathbf{x}$, obtém-se a seguinte expressão para o cálculo de $\nabla J_{k}^{\mathbb{S}}(\mathbf{y})$ em função de $\nabla J_{k}^{\mathbb{S}}(\mathbf{x})$

$$
\nabla J_{k}^{\mathbb{S}}(\mathbf{y})=\nabla J_{k}^{\mathbb{S}}(\mathbf{x})+2\left(\mathbf{A}_{k}\right)_{\mathbb{S}, \mathbb{S}} \Delta \mathbf{x}
$$

No caso particular em que $\Delta \mathbf{x}=-x_{i} \mathbf{u}_{i}$, com $\mathbf{u}_{i}=(\mathbf{I})_{i}, 4$-16 e 4-17 podem ser respectivamente simplificadas como

$$
\begin{gathered}
J_{k}^{\mathbb{S}}(\mathbf{y})=J_{k}^{\mathbb{S}}(\mathbf{x})-\Re\left\{x_{i}^{*}\left(\nabla J_{k}^{\mathbb{S}}(\mathbf{x})\right)_{i}\right\}+\left(\left(\mathbf{A}_{k}\right)_{\mathbb{S}, \mathbb{S}}\right)_{i, i}\left|x_{i}\right|^{2} \\
\nabla J_{k}^{\mathbb{S}}(\mathbf{y})=\nabla J_{k}^{\mathbb{S}}(\mathbf{x})-2 x_{i}\left(\left(\mathbf{A}_{k}\right)_{\mathbb{S}, \mathbb{S}}\right)_{i}
\end{gathered}
$$

onde $\left(\left(\mathbf{A}_{k}\right)_{\mathbb{S}, \mathbb{S}}\right)_{i}$ corresponde à i-ésima coluna de $\left(\mathbf{A}_{k}\right)_{\mathbb{S}, \mathbb{S}}$.

Dessa forma, o algoritmo de Decimação Iterativa pode ser reescrito conforme o Algoritmo 4.2.

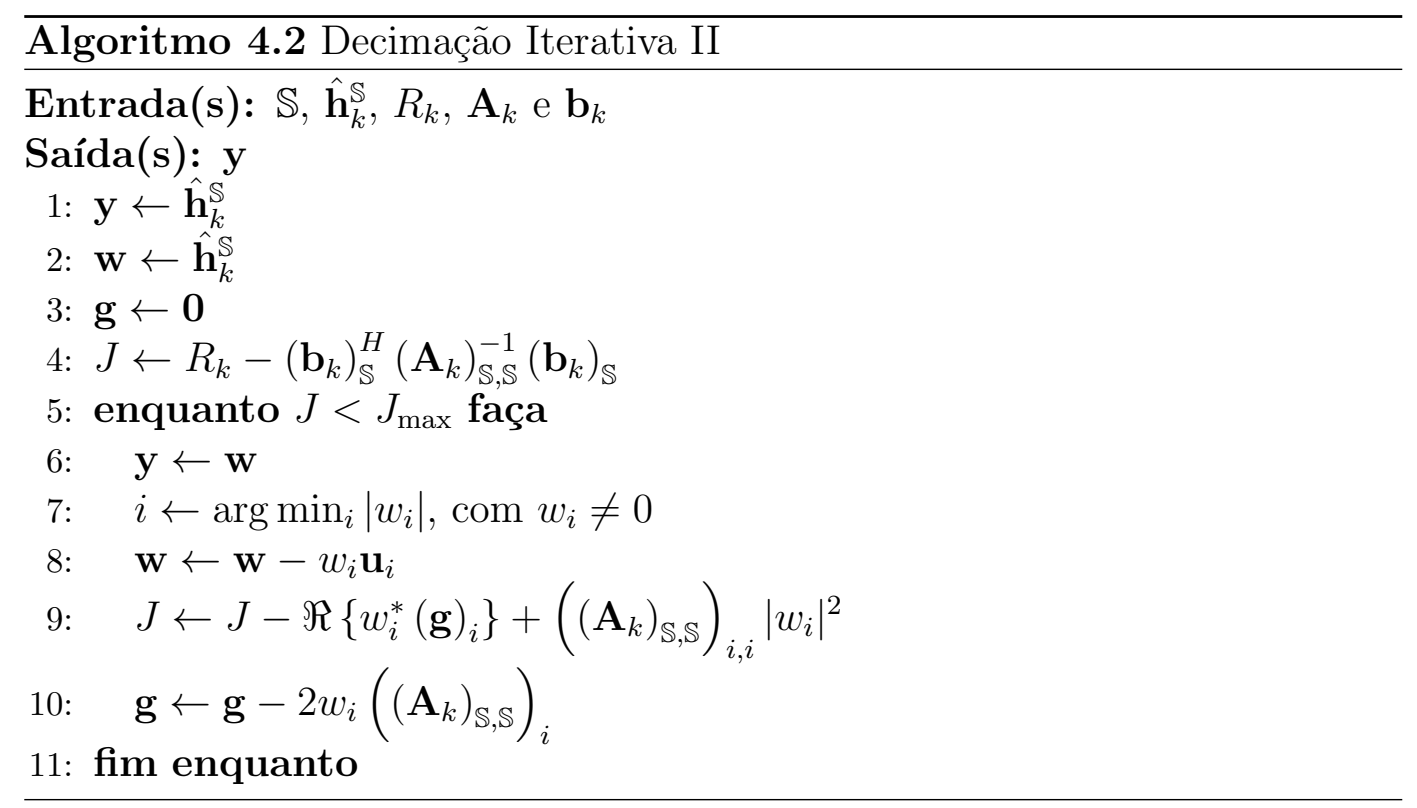

Considerando $k$ suficientemente grande para que seja possível considerar $\left(\mathbf{A}_{k}\right)_{\mathbb{S}, \mathbb{S}} \approx \mathbf{I}$ e empregando 3-6 na expressão $\mathbf{y}=\hat{\mathbf{h}}_{k}^{\mathbb{S}}+\Delta \mathbf{h}$, tem-se que

$$
J_{k}^{\mathbb{S}}(\mathbf{y}) \approx J_{k}(\mathbb{S})+\|\Delta \mathbf{h}\|^{2} \quad k \gg 0
$$

A equação 4-20 pode ser utilizada para o cálculo aproximado de $J_{k}(\mathbf{y})$ quando $k \gg 1$. Nesse caso, o algoritmo de decimação iterativa pode ser simplificado na forma do Algoritmo 4.3. 


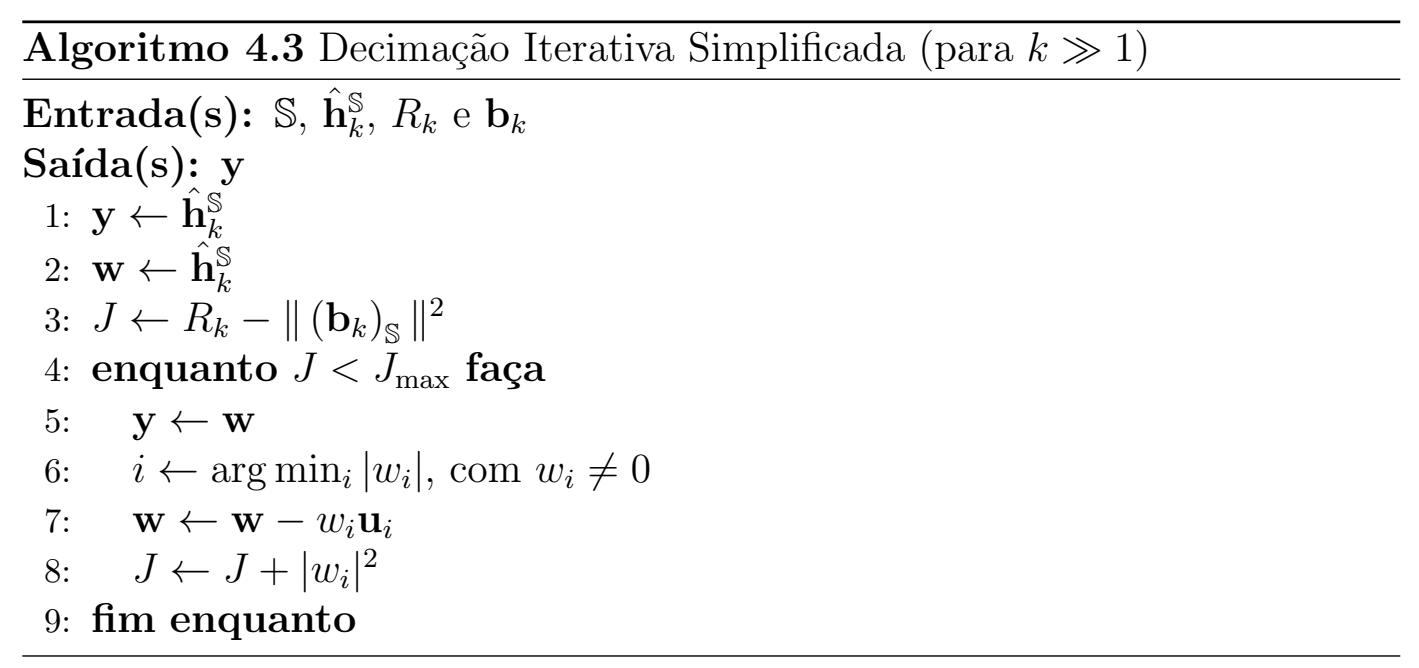

\subsection{3}

\section{Minimização linear}

O procedimento de Minimização Linear, proposto nesta subseção, consiste em $\operatorname{minimizar} J_{k}^{\mathbb{S}}(\mathbf{y})$ restrita à reta $\mathbf{y}=\kappa \mathbf{x}, \kappa \in \mathbb{R}$.

Seja $J_{k}^{\mathbb{S}}(\mathbf{x}, \kappa)=J_{k}^{\mathbb{S}}(\kappa \mathbf{x})$. Usando $3-6, J_{k}^{\mathbb{S}}(\mathbf{x}, \kappa)$ pode ser expressa como

$$
J_{k}^{\mathbb{S}}(\mathbf{x}, \kappa)=R_{k}-2 \kappa \Re\left[\left(\mathbf{b}_{k}\right)_{\mathbb{S}}^{H} \mathbf{x}\right]+\kappa^{2} \mathbf{x}^{H}\left(\mathbf{A}_{k}\right)_{\mathbb{S}, \mathbb{S}} \mathbf{x}
$$

Observando 4-21, verifica-se que $J_{k}^{\mathbb{S}}(\mathbf{x}, \kappa)$ é função quadrática em $\kappa$. Além disso, como $\left(\mathbf{A}_{k}\right)_{\mathbb{S}, \mathbb{S}}$ é matriz positiva-definida, então $\mathbf{x}^{H}\left(\mathbf{A}_{k}\right)_{\mathbb{S}, \mathbb{S}} \mathbf{x}>0 \forall \mathbf{x}$ e, portanto, $J_{k}^{\mathbb{S}}(\mathbf{x}, \kappa)$ é convexa em $\kappa$. Logo, dado $\mathbf{x}$, existe um único valor $\kappa_{\mathbf{x}}$ de $\kappa$ que minimiza $J_{k}^{\mathbb{S}}(\mathbf{x}, \kappa)$. Derivando $J_{k}^{\mathbb{S}}\left(\mathbf{x}, \kappa_{\mathbf{x}}\right)$ em relação a $\kappa_{\mathbf{x}}$ e igualando a zero, verifica-se que

$$
\kappa_{\mathbf{x}}=\frac{\Re\left[\left(\mathbf{b}_{k}\right)_{\mathbb{S}}^{H} \mathbf{x}\right]}{\mathbf{x}^{H}\left(\mathbf{A}_{k}\right)_{\mathbb{S}, \mathbb{S}} \mathbf{x}}
$$

Portanto, o vetor $\mathbf{y}$ que minimiza $J_{k}^{\mathbb{S}}(\mathbf{y})$ na direção de $\mathbf{x}$ é dada por

$$
\mathbf{y}=\kappa_{\mathbf{x}} \mathbf{x}
$$

Convém ressaltar que $L_{0}(\mathbf{y})=L_{0}(\mathbf{x})$. Portanto, o vetor $\mathbf{y}$ produzido pela minimização linear possui a mesma esparsidade que o vetor original x, medida através da função $L_{0}($.$) .$

O emprego da etapa de Minimização Linear no Método de Encolhimentos Sucessivos é opcional e seu propósito é reduzir o esforço computacional na etapa consecutiva de despolarização. 


\section{5 .4}

\section{Despolarização}

A despolarização consiste no cálculo da estimativa LS, associada ao suporte $\mathbb{S}$ obtido na etapa de decimação. O cálculo da estimativa LS associada ao suporte $\mathbb{S}$ é dado por 3-9, repetida aqui por conveniência

$$
\hat{\mathbf{h}}_{k}^{\mathbb{S}}=\left(\mathbf{A}_{k}\right)_{\mathbb{S}, \mathbb{S}}^{-1}\left(\mathbf{b}_{k}\right)_{\mathbb{S}}
$$

Seja $\hat{\mathbf{h}}_{k}$ a estimativa esparsa associada ao suporte $\mathbb{S}$, interpolada para o suporte pleno $\mathbb{L}$, dada por

$$
\hat{\mathbf{h}}_{k}=(\mathbf{I})_{\mathbb{L}, \mathbb{S}} \hat{\mathbf{h}}_{k}^{\mathbb{S}}
$$

onde $(\mathbf{I})_{\mathbb{L}, \mathbb{S}}$ é a matriz formada pelas colunas da matriz-identidade $\mathbf{I}_{\mathbb{L}, \mathbb{L}}$ cujos índices pertencem a $\mathbb{S}$.

O fato dessa técnica ser denominada despolarização justifica-se pelo fato de que, como para todo suporte $\mathbb{S}, \hat{\mathbf{h}}_{k}^{\mathbb{S}} \stackrel{a . s .}{\longrightarrow}(\mathbf{h})_{\mathbb{S}}$, verifica-se que

$$
\mathbb{S} \supseteq \mathbb{S}_{\mathbf{h}} \Rightarrow \hat{\mathbf{h}}_{k} \stackrel{a . s .}{\longrightarrow} \mathbf{h}
$$

Portanto, se $\mathbb{S} \supseteq \mathbb{S}_{\mathbf{h}}$, então $\hat{\mathbf{h}}_{k}=(\mathbf{I})_{\mathbb{L}, \mathbb{S}} \hat{\mathbf{h}}_{k}^{\mathbb{S}}$ é uma estimativa consistente e despolarizada da resposta ao impulso verdadeira $\mathbf{h}$ do sistema estimado.

\subsection{5}

\section{Algoritmo proposto}

A etapa de decimação iterativa provoca o aumento do custo LS até o limite $J_{\max }$ ser ultrapassado. Por outro lado, as etapas de minimização linear e de despolarização puxam o custo LS novamente para baixo e, se a redução é suficiente para colocar o custo LS novamente abaixo do limiar $J_{\max }$, repetese o procedimento. Dessa forma, o método de Encolhimentos Sucessivos gera uma sequência de suportes $\left\{\mathbb{S}^{(i)} \mid \mathbb{S}^{(i)} \supset \mathbb{S}^{(i+1)}\right\}$ e suas respectivas estimativas $\left\{\hat{\mathbf{h}}_{k}^{\mathbb{S}^{(i)}}, i=1,2, \ldots\right\}$, enquanto a condição $J\left(\mathbb{S}^{(i)}\right)<J_{\max }$ é satisfeita. O método proposto é sumarizado no Algoritmo 4.4.

O Método de Encolhimentos Sucessivos explora o fato de que a sequência $\left\{J_{k}\left(\mathbb{S}^{(i)}\right) \mid \mathbb{S}^{(i+1)} \subset \mathbb{S}^{(i)}\right\}$ é monótona não-decrescente, conforme demonstrado pelo Teorema 3.1. A monotonicidade da sequência de custos LS gerada pelo método garante sua convergência.

A Figura 4.2 ilustra o funcionamento do Método de Encolhimentos Sucessivos. Embora a sequência dos custos LS associada aos vetores obtidos na etapa de 


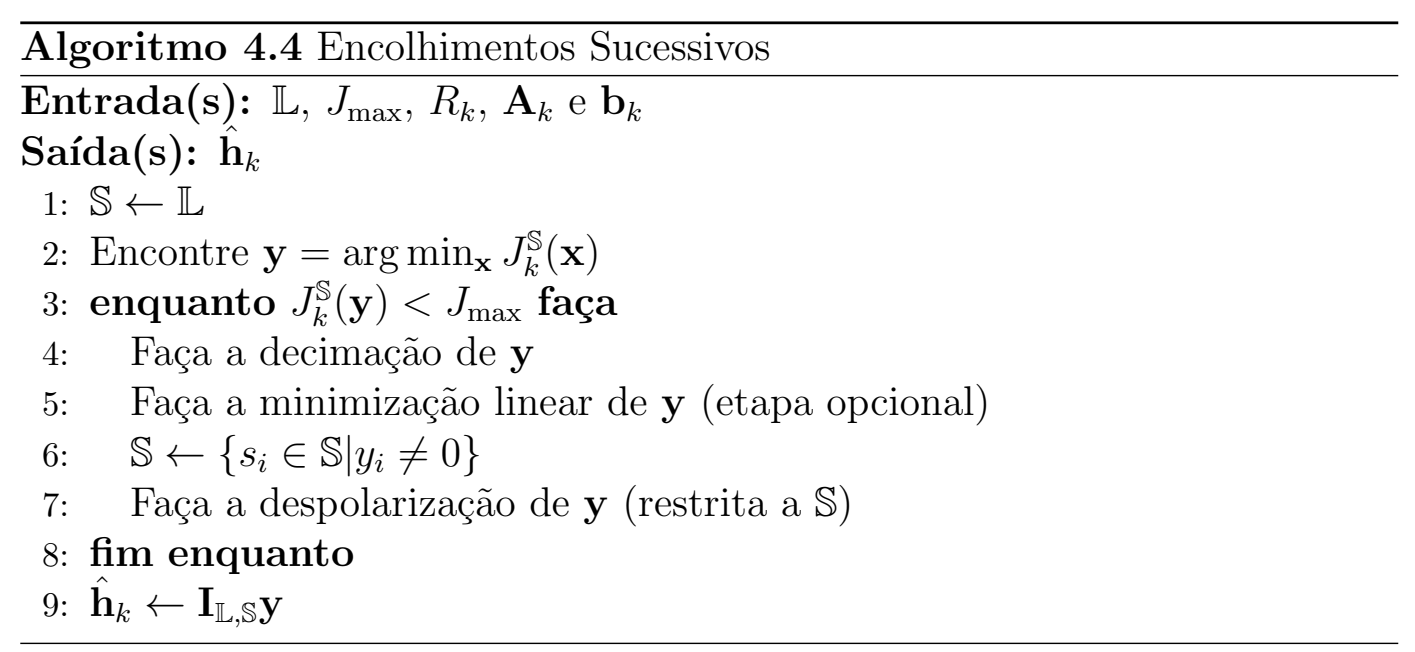

decimação não seja monótona crescente, é possível constatar que ela é limitada inferiormente pela sequência dos mínimos custos LS, associada aos suportes dos vetores obtidos durante a decimação, que é monótona crescente.

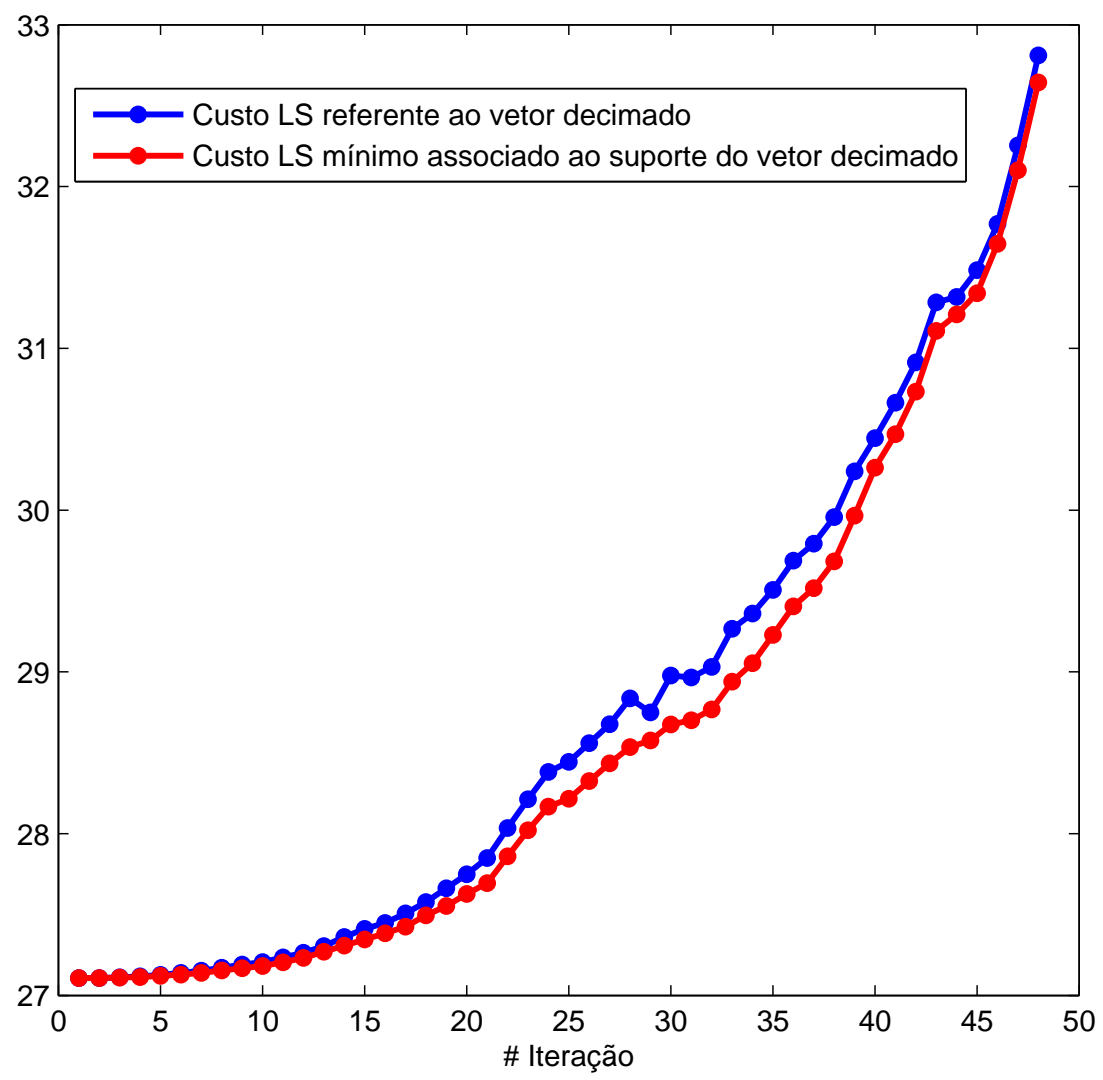

Figura 4.2: Exemplo do funcionamento do Método de Encolhimentos Sucessivos relativa à estimação de uma realização de um sistema $\operatorname{MESS}(50,10)$, com $K=5, \mathrm{SINR}=3 \mathrm{~dB}, N=16$ e $\lambda=1,2$ 


\subsection{6}

\section{Custo computacional}

O custo computacional do Método de Encolhimentos Sucessivos consiste na soma dos custos computacionais de todas as decimações, minimizações lineares e despolarizações realizadas.

O custo computacional de cada iteração da etapa de Decimação Iterativa corresponde à soma dos custos computacionais das equações 4-16 e 4-17, que é aproximadamente $3 \times|\mathbb{S}|+8$ flops apenas. Considerando que $\mathbb{S}^{(1)}=\mathbb{L}$ e supondo a ocorrência de um encolhimento correto $\left(\hat{\mathbb{S}}=\mathbb{S}_{\mathbf{h}}\right)$, então o custo computacional total da Decimação Iterativa é menor ou igual a $\left(|\mathbb{L}|-\left|\mathbb{S}_{\mathbf{h}}\right|\right) \times$ $(3 \times|\mathbb{L}|+8)$, que é $\mathcal{O}\left(|\mathbb{L}|^{2}\right)$, enquanto que o custo computacional do cálculo da estimativa LS com suporte reduzido é $\mathcal{O}\left(\left|\mathbb{S}_{\mathbf{h}}\right|^{3}\right)$.

\subsection{7}

\section{Análise de desempenho}

As Figuras 4.3, 4.4, 4.5 e 4.6 mostram o desempenho do método de Encolhimentos Sucessivos, $\operatorname{com} \lambda=1,05$, comparado com o desempenho das estimações LS full-support e LS oráculo, em função da quantidade $K$ de blocos da sequência de treinamento e $\mathrm{SINR}=5,10,15,20,25$ e $30 \mathrm{~dB}$ respectivamente, avaliado sobre $Q=1000$ realizações independentes de um sistema $\operatorname{MESS}(100,10)$ para cada valor de SINR. O comprimento escolhido para os blocos de treinamento foi $\mathrm{N}=16$ e seus elementos foram sorteados de $-1,+1$ de forma equiprovável. As Figuras 4.9, 4.10, 4.11 e 4.12 apresentam resultados obtidos com sistema MESS(100,20), mantendo-se os demais parâmetros de simulação inalterados. Nos gráficos superiores, são mostrados os desempenhos dos algoritmos citados em termos de NMSD. Nos gráficos inferiores, são mostradas as probabilidades de ocorrência dos possíveis eventos de encolhimento, discutidos na seção 4.2.1. 

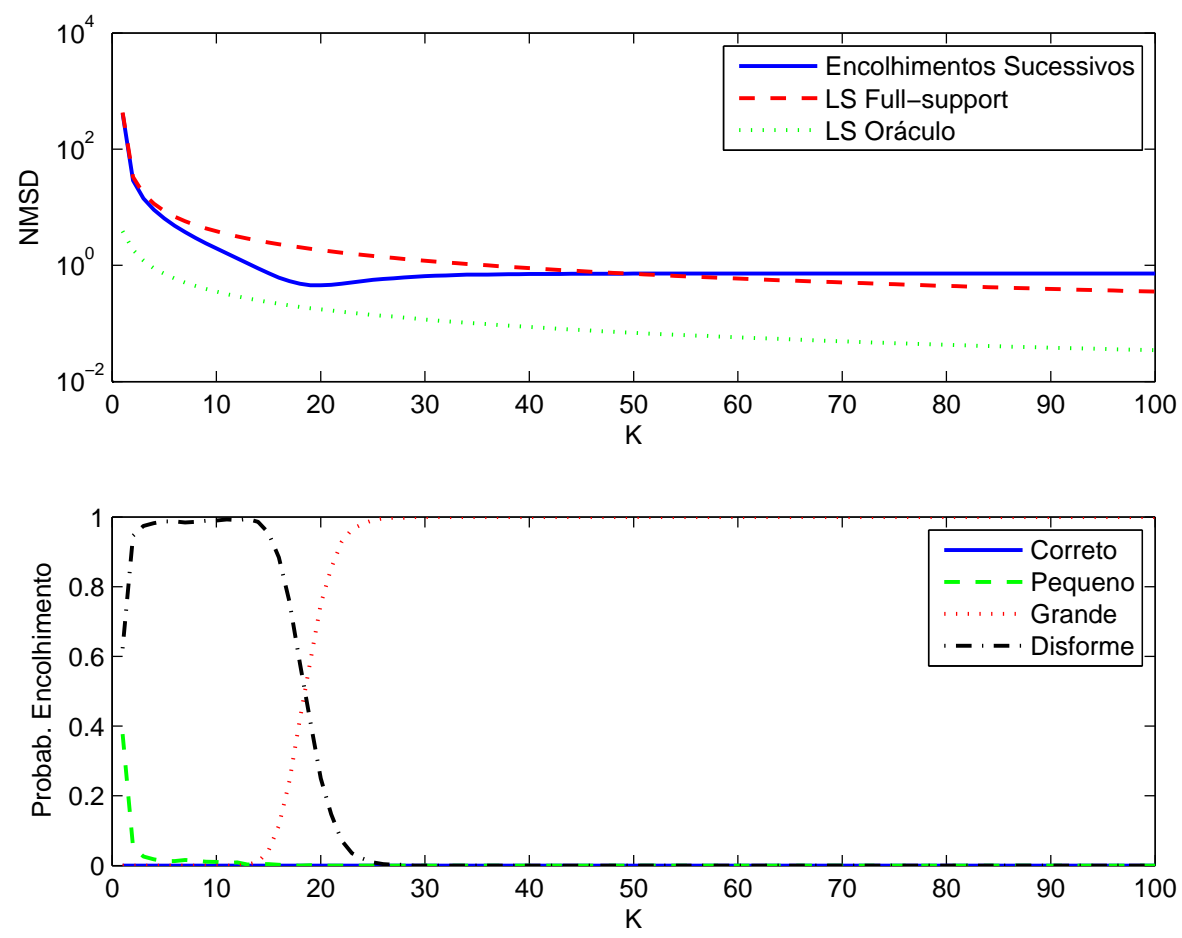

Figura 4.3: Enc. Suc.: $\operatorname{SINR=5dB,~} \operatorname{MESS}(100,10), \lambda=1,05, N=16$ e $Q=1000$
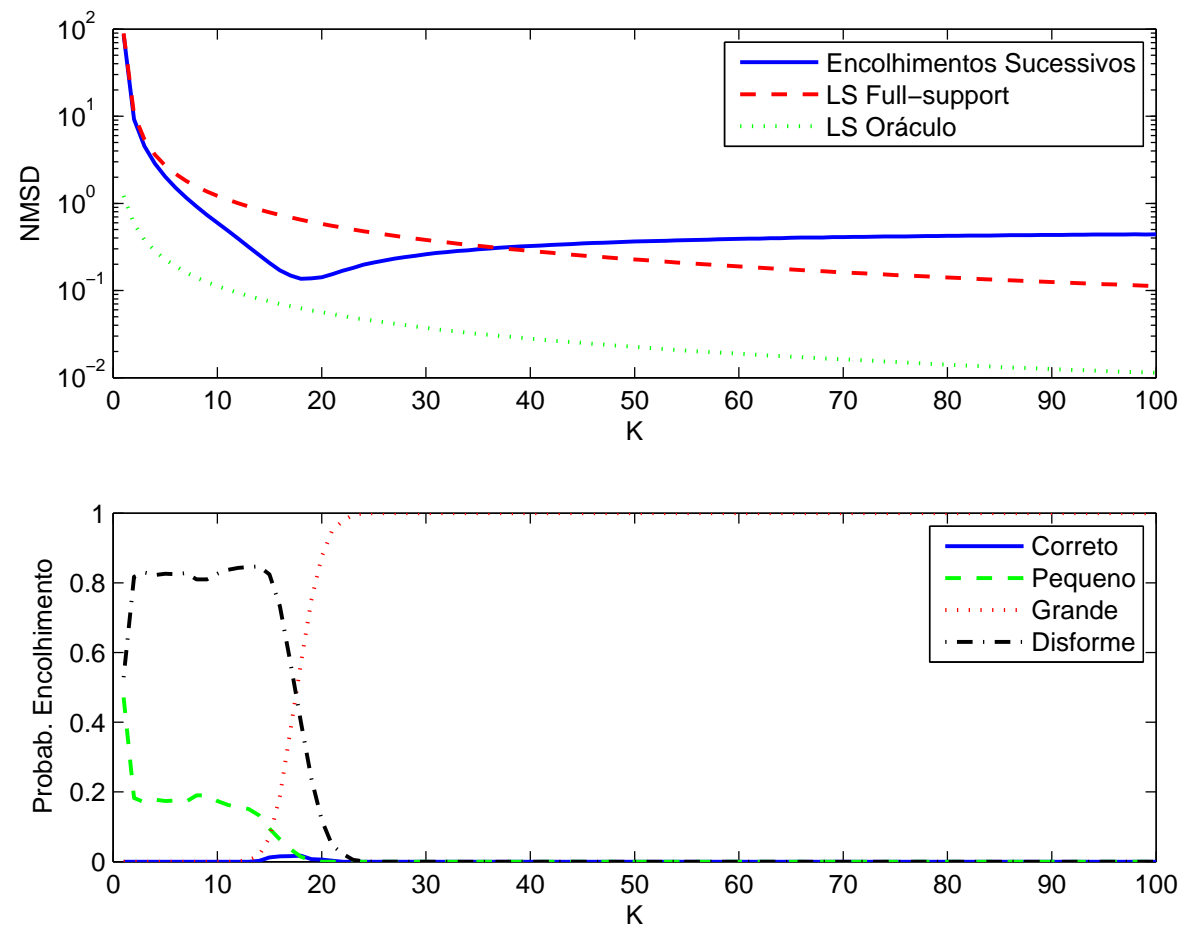

Figura 4.4: Enc. Suc.: SINR=10dB, $\operatorname{MESS}(100,10), \lambda=1,05, \mathrm{~N}=16$ e $\mathrm{Q}=1000$ 

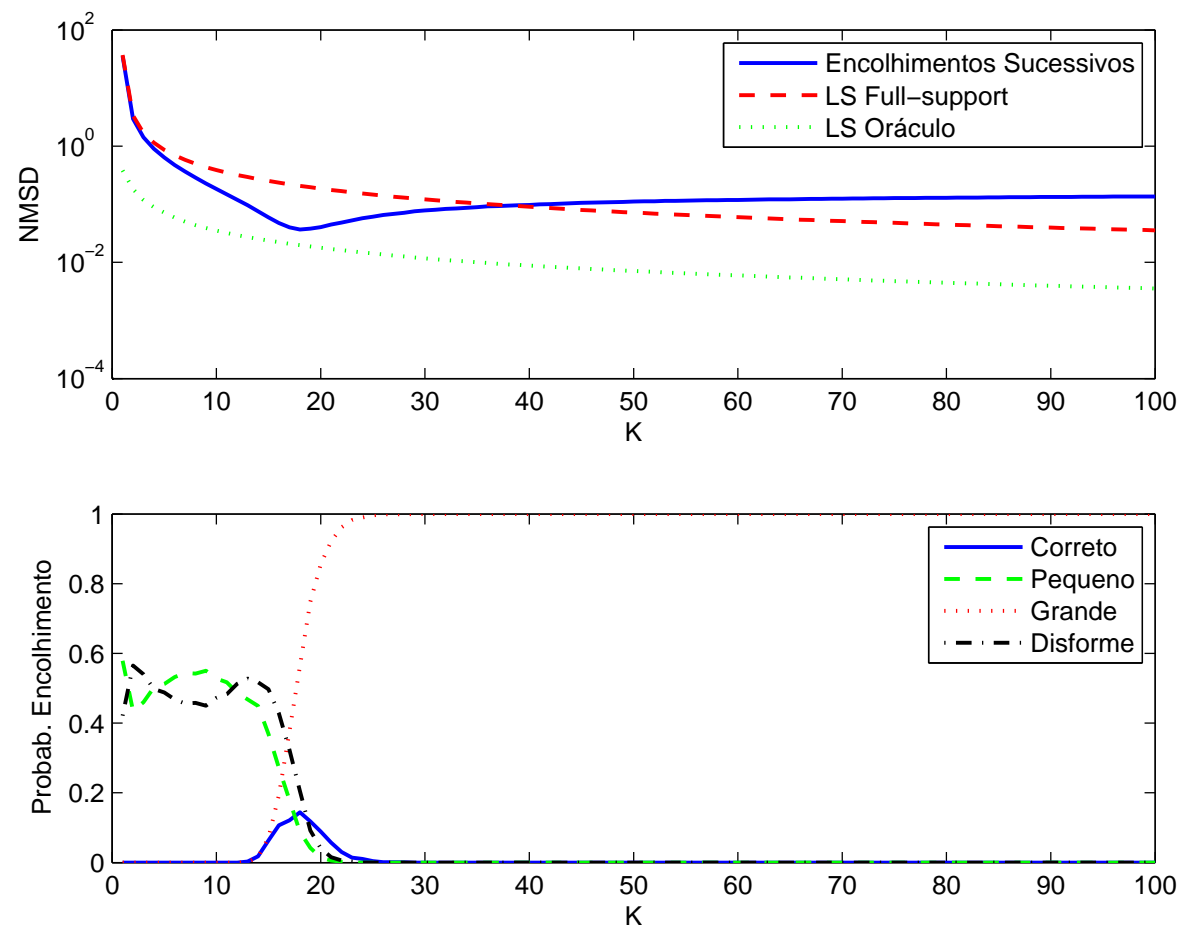

Figura 4.5: Enc. Suc.: $\operatorname{SINR}=15 d B, \operatorname{MESS}(100,10), \lambda=1,05, N=16$ e $Q=1000$
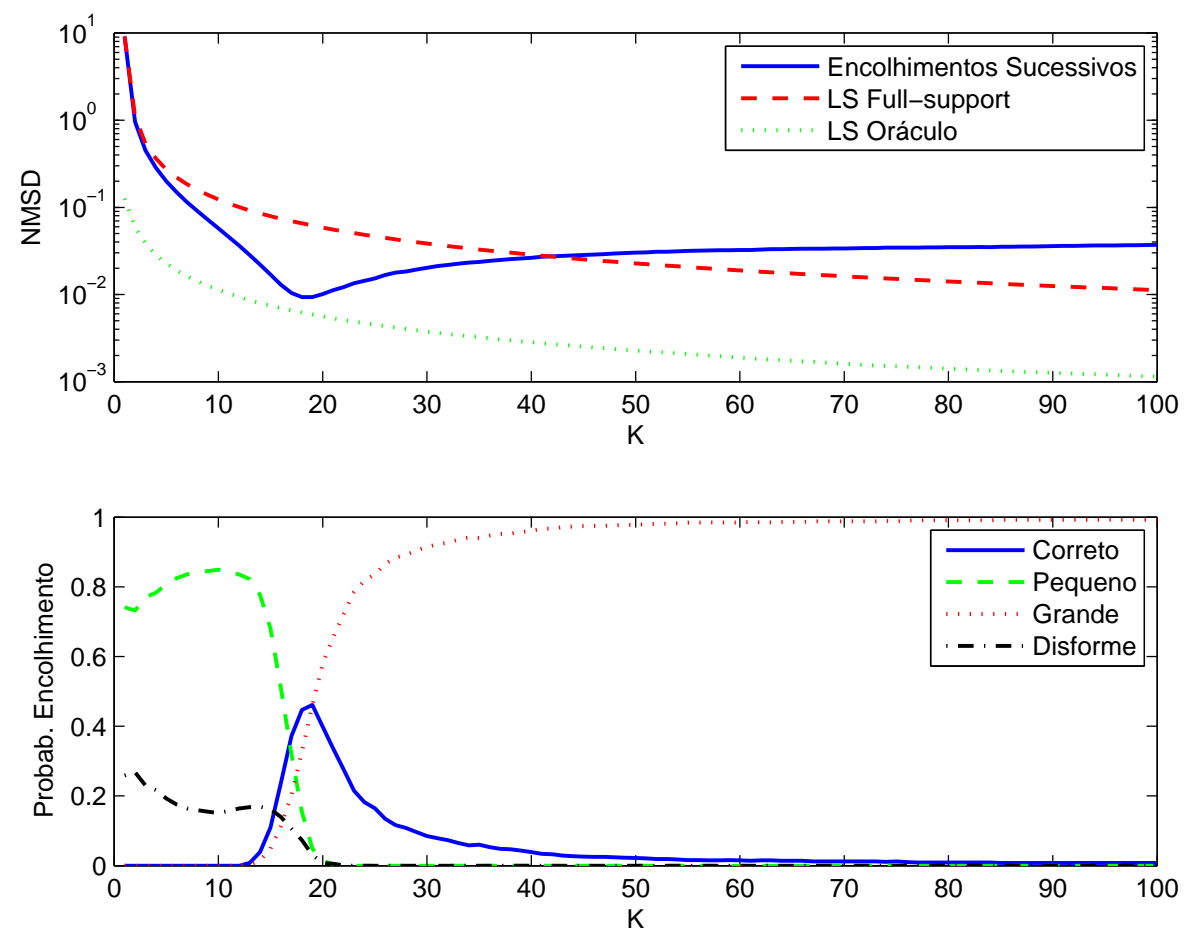

Figura 4.6: Enc. Suc.: $\operatorname{SINR}=20 d B, \operatorname{MESS}(100,10), \lambda=1,05, N=16$ e $Q=1000$ 

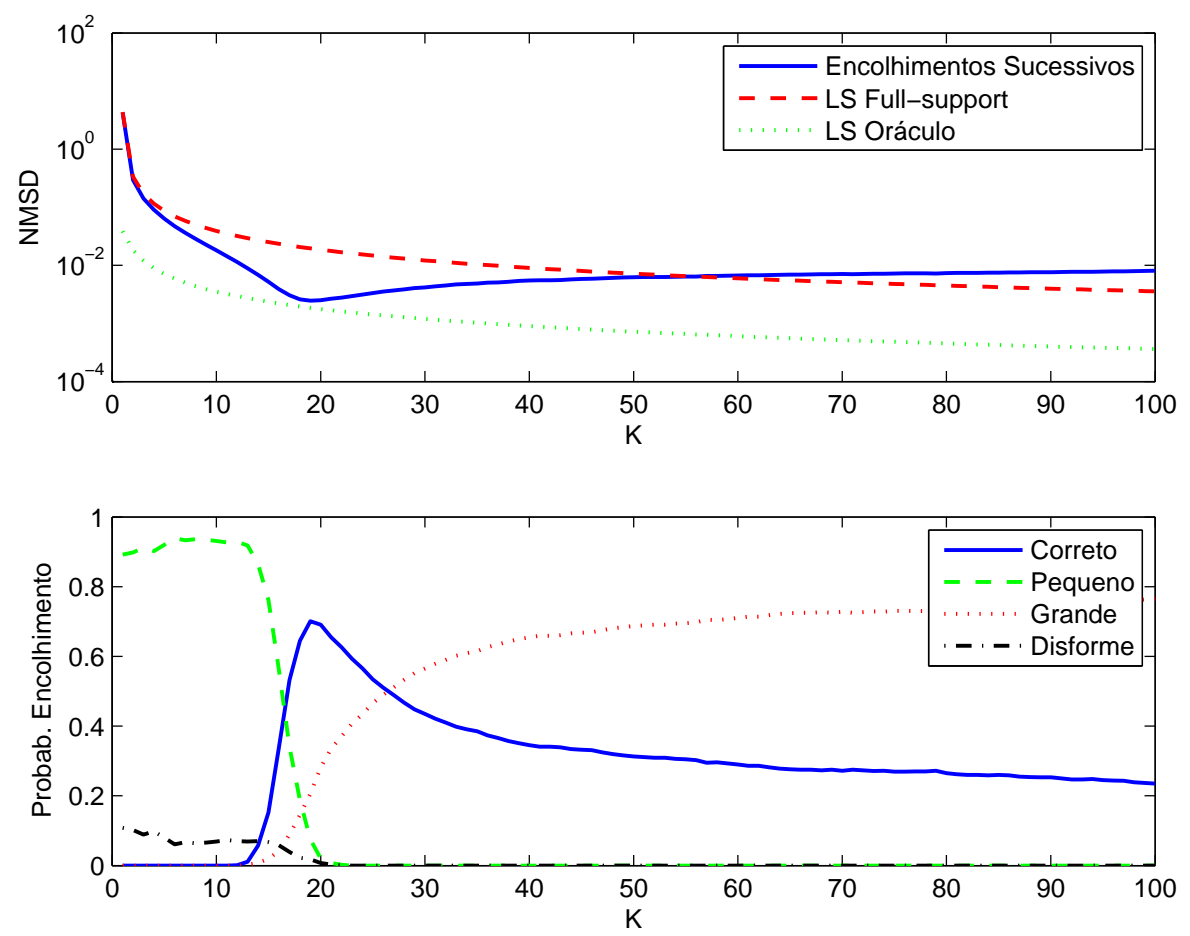

Figura 4.7: Enc. Suc.: $\operatorname{SINR}=25 d B, \operatorname{MESS}(100,10), \lambda=1,05, N=16$ e $Q=1000$
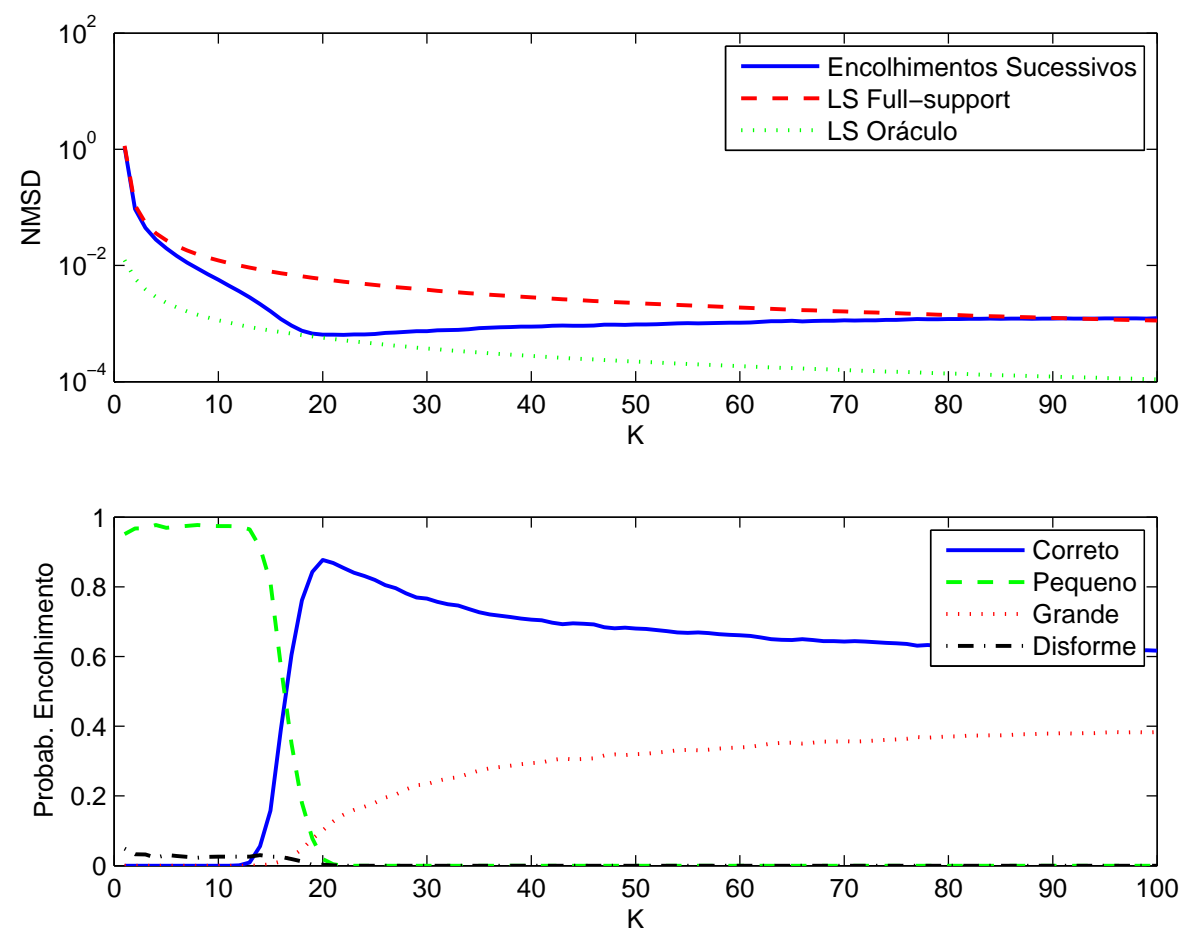

Figura 4.8: Enc. Suc.: $\operatorname{SINR}=30 d B, \operatorname{MESS}(100,10), \lambda=1,05, N=16$ e $Q=1000$ 

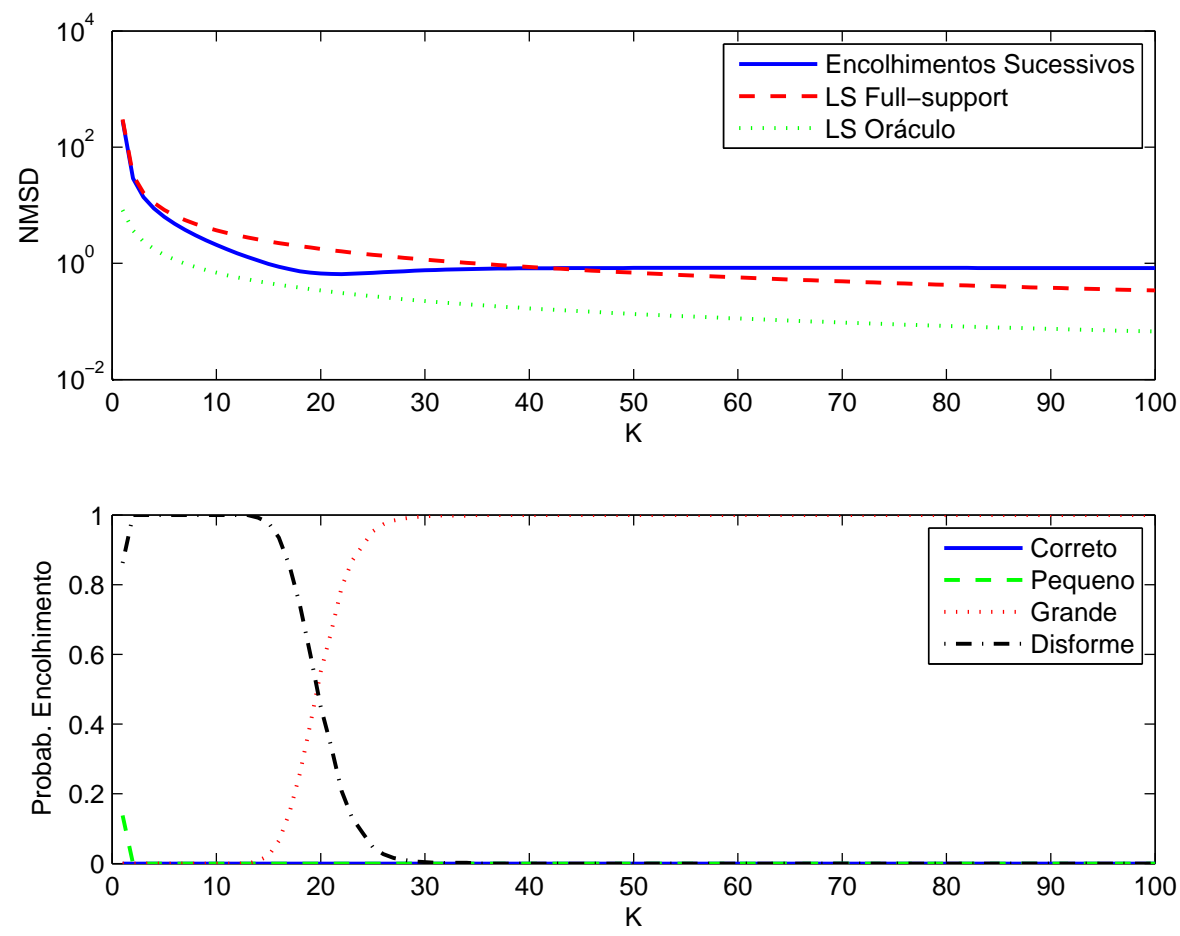

Figura 4.9: Enc. Suc.: SINR=5dB, MESS(100,20), $\lambda=1,05, N=16$ e $Q=1000$
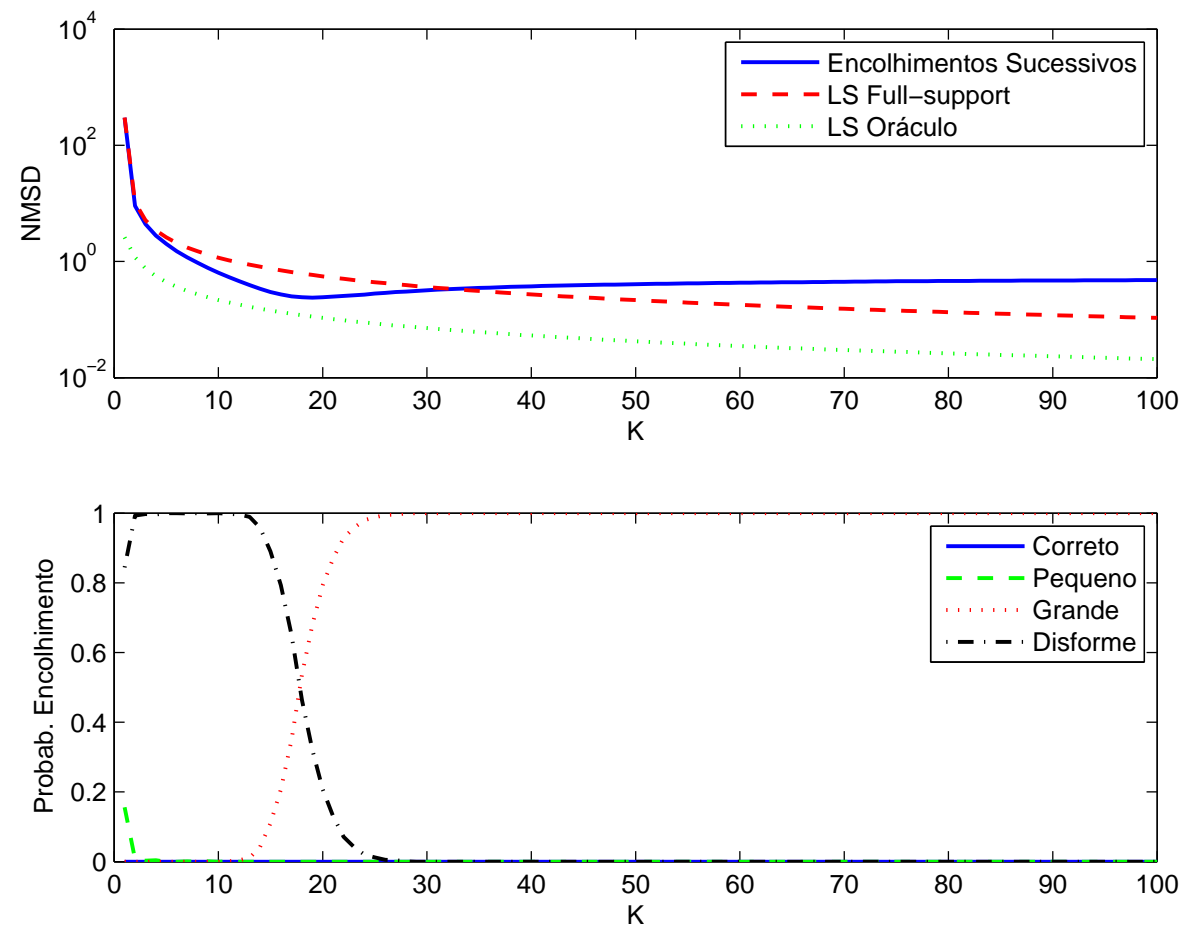

Figura 4.10: Enc. Suc.: $\operatorname{SINR}=10 d B, \operatorname{MESS}(100,20), \lambda=1,05, N=16$ e Q=1000 

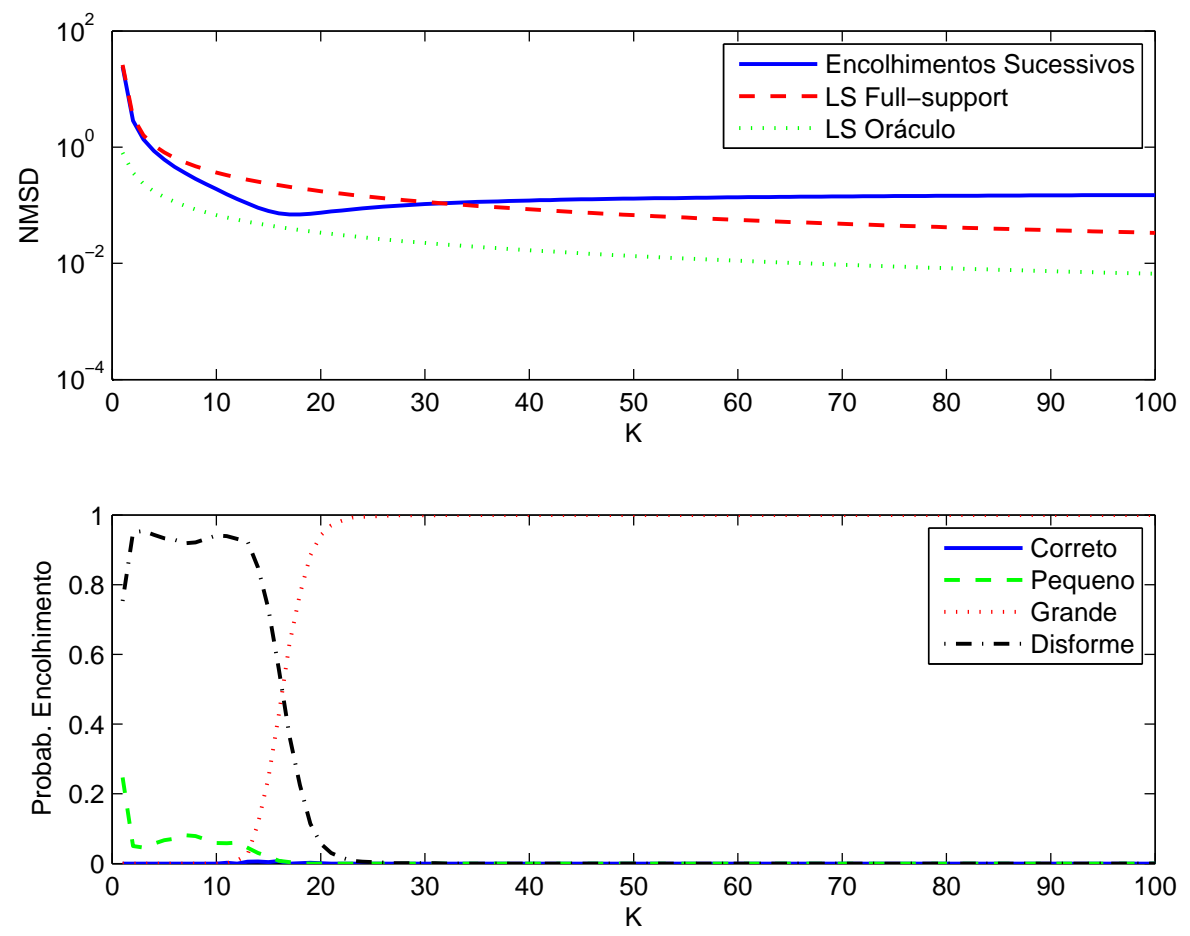

Figura 4.11: Enc. Suc.: $\operatorname{SINR}=15 d B, \operatorname{MESS}(100,20), \lambda=1,05, N=16$ e Q=1000
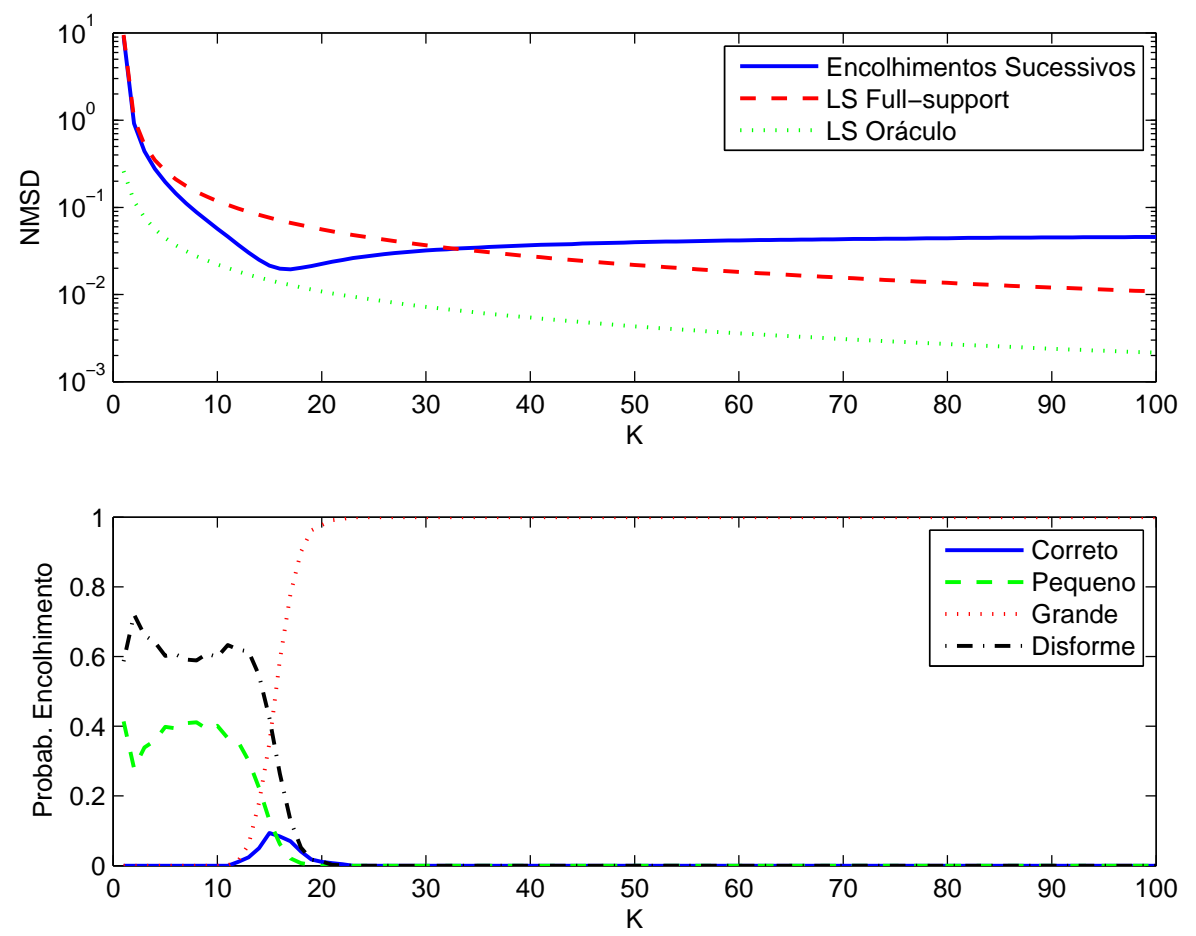

Figura 4.12: Enc. Suc.: $\operatorname{SINR}=20 d B, \operatorname{MESS}(100,20), \lambda=1,05, N=16$ e $Q=1000$ 

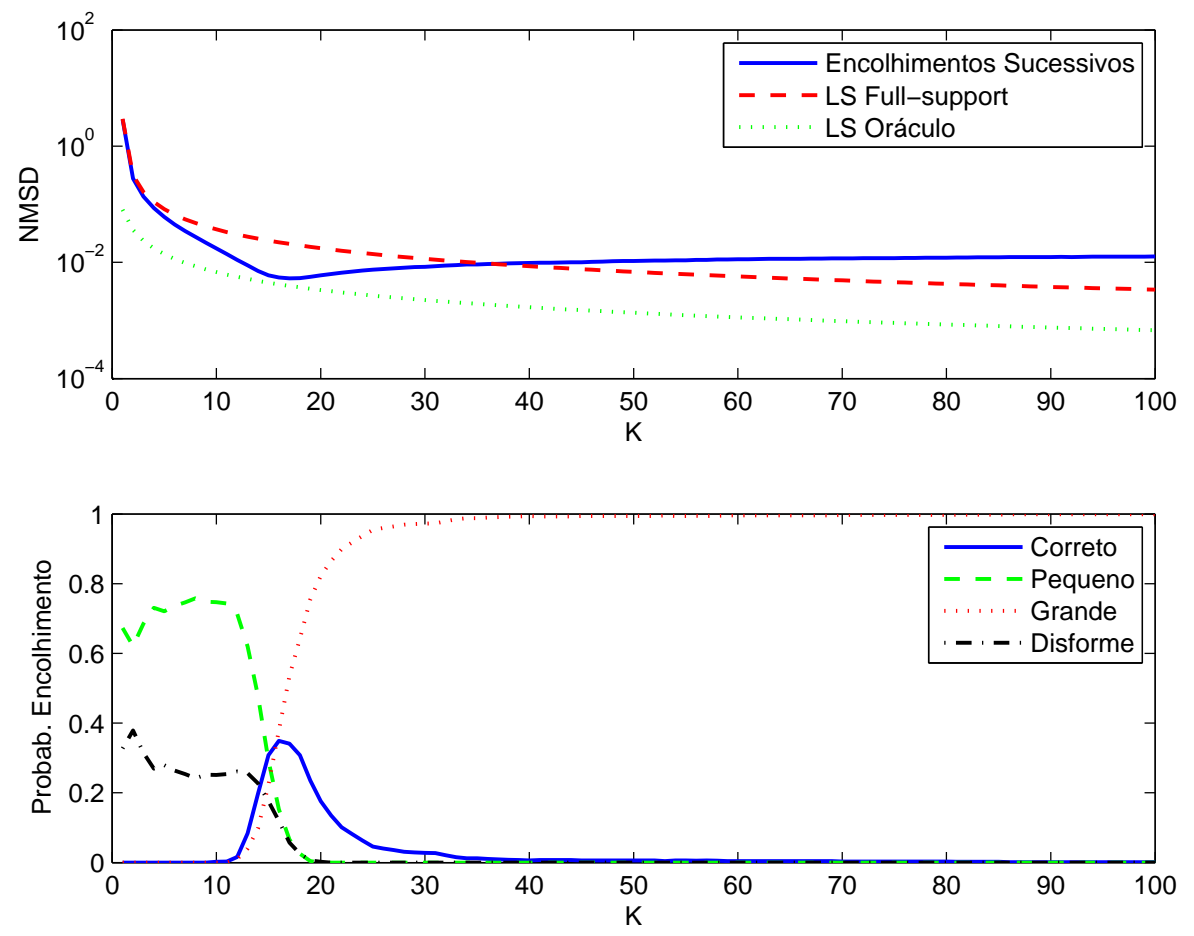

Figura 4.13: Enc. Suc.: $\operatorname{SINR=25dB,~} \operatorname{MESS}(100,20), \lambda=1,05, \mathrm{~N}=16$ e $\mathrm{Q}=1000$
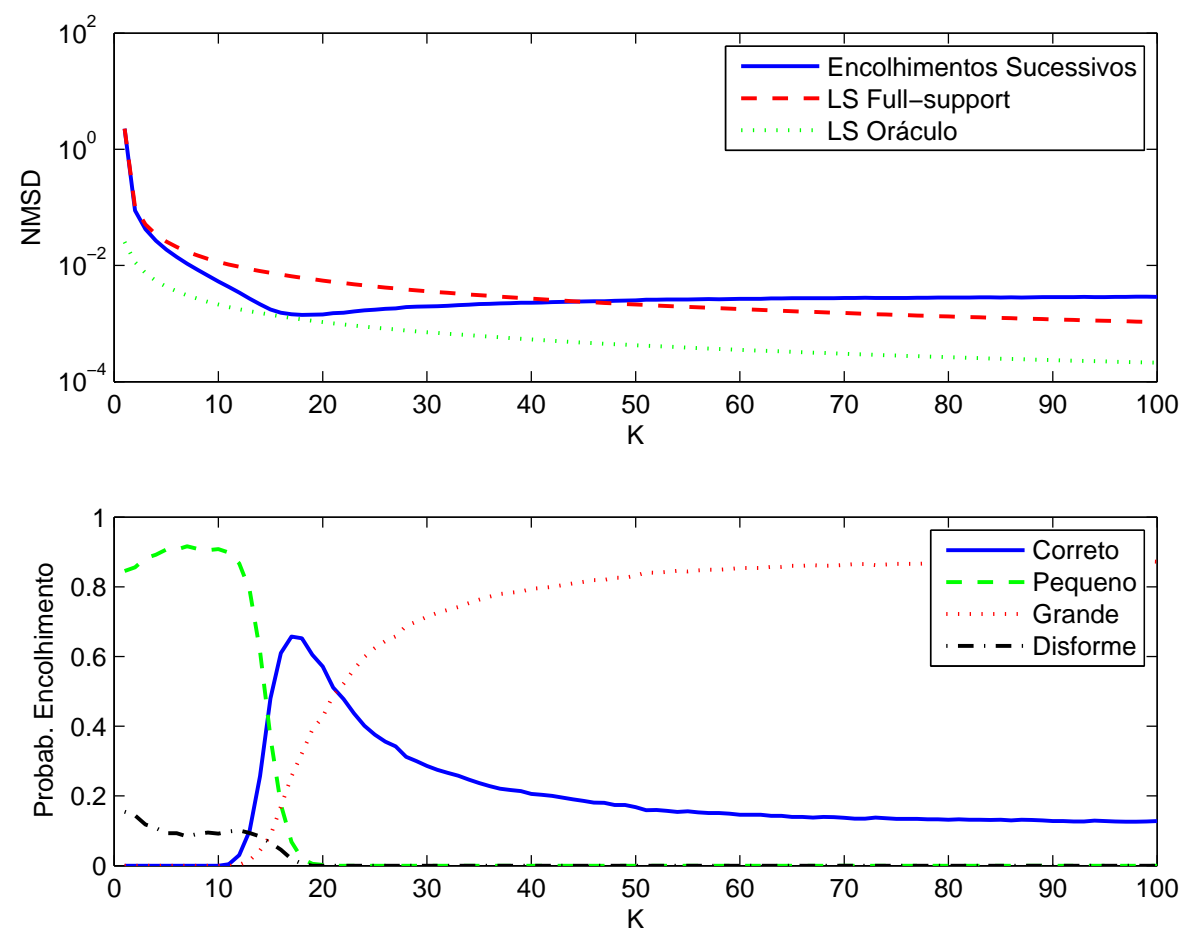

Figura 4.14: Enc. Suc.: $\operatorname{SINR}=30 d B, \operatorname{MESS}(100,20), \lambda=1,05, N=16$ e $Q=1000$ 


\section{6}

\section{Método de Expansões Sucessivas}

\subsection{1}

\section{Introdução}

O Método Expansões Sucessivas, proposto nesta seção, é um método sub-ótimo de estimação de sistemas esparsos baseado no algoritmo de Matching Pursuit (MP) (6). O algoritmo de MP constrói, a partir de um vetor $\mathbf{x}$, uma projeção $\hat{\mathbf{x}}$ em um sub-espaço varrido por um conjunto $\mathcal{D}=\left\{\mathbf{u}_{1}, \mathbf{u}_{2}, \ldots, \mathbf{u}_{P}\right\}$ de vetores de base, todos com norma unitária, mas não necessariamente ortogonais entre si. Definindo o resíduo $\xi^{(i)}=\mathbf{x}-\hat{\mathbf{x}}^{(i)}$ e começando com $\hat{\mathbf{x}}^{(0)}=\mathbf{0}$, este procedimento é realizado iterativamente, de forma que, na $i$-ésima iteração $(i=1,2, \ldots)$, $\hat{\mathbf{x}}^{(i)}=\sum_{j=1}^{i} x^{(j)} \mathbf{u}^{(j)}$, onde $\mathbf{u}^{(i)} \in \mathcal{D}$ e $\mathbf{u}^{(i)} \neq \mathbf{u}^{(i-1)}$, até que um critério de parada seja atendido (por exemplo, $\left\|\xi^{(i)}\right\|<\xi_{\max }$ ). Em cada iteração, $\mathbf{u}^{(i)}=\arg \max _{\mathbf{v} \in \mathcal{D}^{(i-1)}}\left|x^{(i)}\right|$, sendo $x^{(i)}=\left(\mathbf{u}^{(i)}\right)^{H} \xi^{(i-1)}$ e $\mathcal{D}^{(i)}=\mathcal{D}^{(i-1)} \backslash\left\{\mathbf{u}^{(i)}\right\}$.

\subsection{2}

\section{Algoritmo proposto}

Considere o problema de estimação da resposta ao impulso de um sistema a partir da expressão em 3-45, repetida a seguir por conveniência

$$
\overline{\mathbf{r}}_{k}=\overline{\mathbf{C}}_{k} \mathbf{h}+\overline{\mathbf{z}}_{k}
$$

onde $\overline{\mathbf{r}}_{k}=\left[\mathbf{r}_{1}^{T} \mathbf{r}_{2}^{T} \ldots \mathbf{r}_{k}^{T}\right]^{T}$ é vetor de dimensão $k M \times 1, \overline{\mathbf{C}}_{k}=\left[\mathbf{C}_{1}^{T} \mathbf{C}_{2}^{T} \ldots \mathbf{C}_{k}^{T}\right]^{T}$ é matriz de dimensão $k M \times L$ e $\overline{\mathbf{z}}_{k}=\left[\mathbf{z}_{1}^{T} \mathbf{z}_{2}^{T} \ldots \mathbf{z}_{k}^{T}\right]^{T}$ é vetor de dimensão $k M \times 1$. O vetor de observação $\overline{\mathbf{r}}_{k}$ pode ser genericamente representado da seguinte forma

$$
\overline{\mathbf{r}}_{k}=\overline{\mathbf{C}}_{k} \mathbf{x}+\xi=x_{1} \overline{\mathbf{c}}_{k, 1}+x_{2} \overline{\mathbf{c}}_{k, 2}+\ldots+x_{L} \overline{\mathbf{c}}_{k, L}+\xi
$$

onde $L$ é a quantidade de colunas de $\overline{\mathbf{C}}_{k}, \overline{\mathbf{c}}_{k, i}$ é a $i$-ésima coluna de $\overline{\mathbf{C}}_{k}, x_{i}$ é o $i$-ésimo elemento de $\mathbf{x}$ e $\xi$ é o resíduo (diferença) entre $\overline{\mathbf{r}}_{k}$ e $\overline{\mathbf{C}}_{k} \mathbf{x}$.

O custo LS é definido em 3-47, repetido aqui por conveniência

$$
J_{k}^{\mathbb{S}}(\mathbf{x})=\frac{1}{k}\left\|\overline{\mathbf{r}}_{k}-\left(\overline{\mathbf{C}}_{k}\right)_{\mathbb{S}} \mathbf{x}\right\|^{2}
$$

Comparando 4-28 e 4-29, verifica-se que, dado x, tem-se que

$$
\|\xi\|^{2}=k J_{k}^{\mathbb{S}}(\mathbf{x})
$$


Portanto, adotando no algoritmo de MP $\mathcal{D}=\left\{\overline{\mathbf{c}}_{k, 1}, \overline{\mathbf{c}}_{k, 2}, \ldots, \overline{\mathbf{c}}_{k, L}\right\}$, substituindo $\xi_{\max }$ por um limiar $J_{\max }$ e monitorando iterativamente $J_{k}($.$) em vez de \|\xi\|$, origina-se o Método de Expansões Sucessivas, descrito no Algoritmo 4.5.

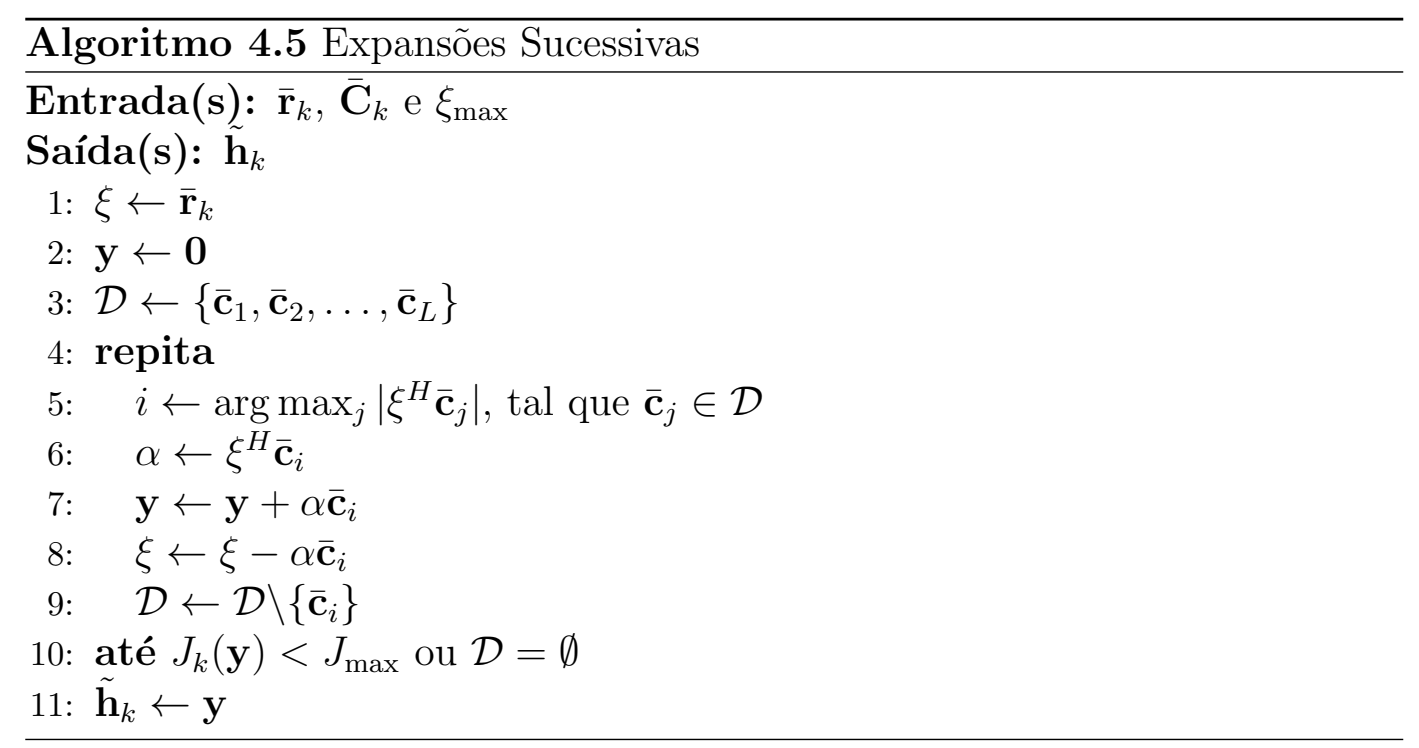

O Algoritmo 4.5 possui a desvantagem de exigir o armazenamento em memória e o empilhamento de todos os vetores de observação $\mathbf{r}_{1}, \mathbf{r}_{2}, \ldots, \mathbf{r}_{k}$ e de todas as sequências de símbolos transmitidos $\mathbf{c}_{1}, \mathbf{c}_{2}, \ldots, \mathbf{c}_{k}$. Devido a essa desvantagem, é proposta a seguir uma segunda versão do método, que apresenta menor custo computacional.

Utilizando 3-9, tem-se que

$$
\mathbf{b}_{k}=\mathbf{A}_{k} \hat{\mathbf{h}}_{k}
$$

Aplicando 3-17 em 4-31, tem-se que

$$
\mathbf{b}_{k}=\mathbf{A}_{k}\left(\mathbf{h}+\boldsymbol{\varepsilon}_{k}\right)=h_{1}\left(\mathbf{A}_{k}\right)_{1}+h_{2}\left(\mathbf{A}_{k}\right)_{2}+\ldots+h_{L}\left(\mathbf{A}_{k}\right)_{L}+\xi
$$

onde

$$
\xi=\mathbf{A}_{k} \varepsilon_{k}
$$

Baseado em 4-32 e utilizando as equações 4-16 e 4-17 para o cálculo iterativo do custo LS à medida em que a estimativa é construída, propõe-se uma segunda versão do método de Expansões Sucessivas, conforme descrito no Algoritmo 4.6 .

O método de Expansões Sucessivas, na versão descrita pelo Algoritmo 4.6, diferencia-se da versão descrita pelo Algoritmo 4.5 no seguinte aspecto fundamental: enquanto que na primeira versão o processamento é realizado diretamente sobre o vetor de observações $\overline{\mathbf{r}}_{k}$, na segunda versão, o processamento é 


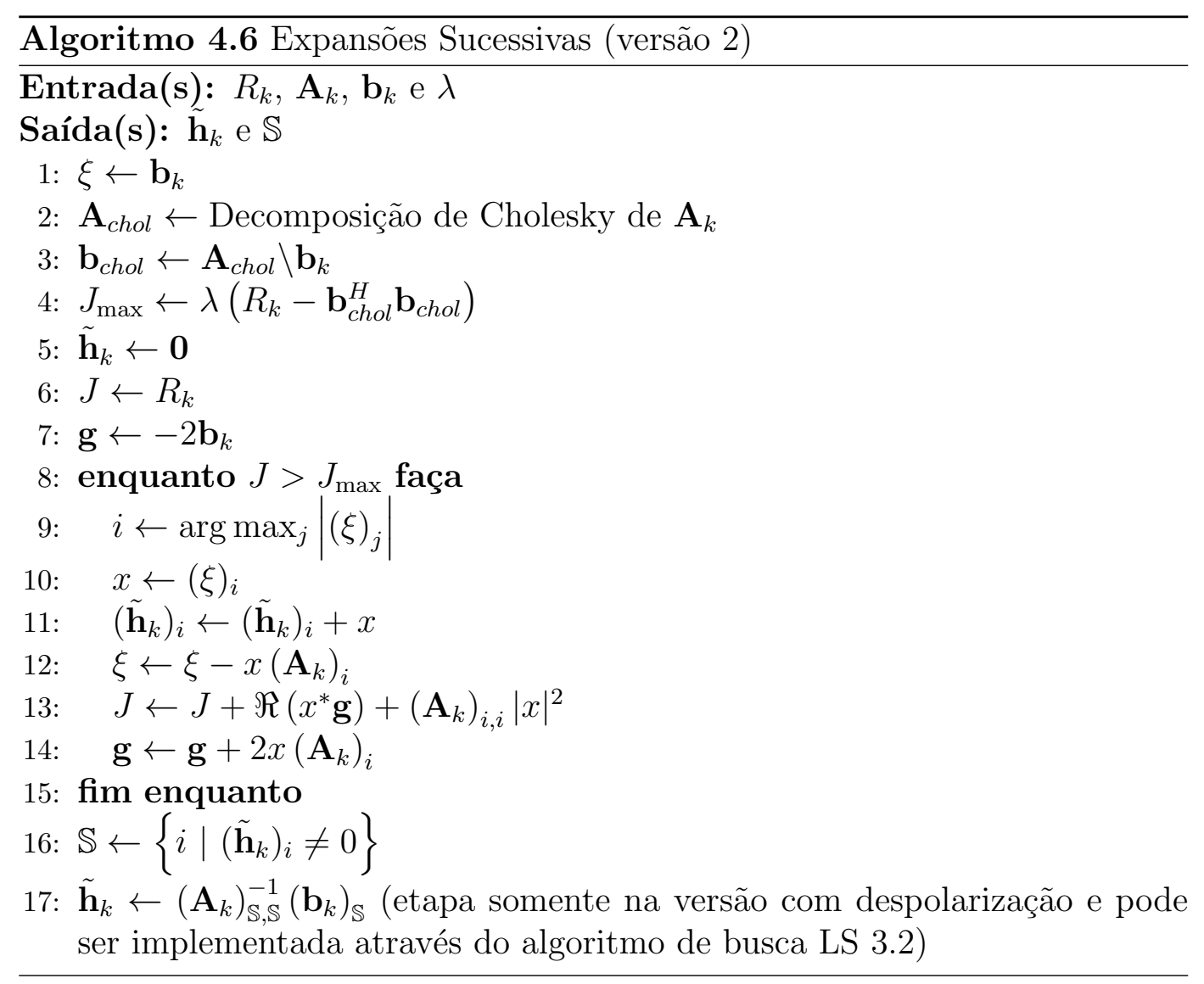

realizado sobre o vetor $\mathbf{b}_{k}$. A etapa opcional de despolarização, quando executada, proporciona um melhor desempenho ao método.

O método de Expansões Sucessivas corresponde ao oposto do método de Encolhimentos Sucessivos, no seguinte aspecto: enquanto o método de Encolhimentos Sucessivos constrói uma estimativa a partir da decimação da estimativa LS, indo em direção ao vetor nulo, o método de Expansões Sucessivas constrói uma estimativa a partir do vetor nulo, indo em direção à estimativa LS.

\subsection{3}

\section{Custo computacional}

O custo computacional de cada iteração do Algoritmo 4.6 é praticamente o mesmo de cada iteração do Algoritmo 4.2 (algoritmo de Decimações Iterativas), haja vista que ambos empregam as mesmas equações (equações 4-18 e 4-19) para atualizar o custo LS à medida em que o vetor estimado é construído. A diferença está no fato de que, admitindo uma expansão correta do suporte $\left(\hat{\mathbb{S}}=\mathbb{S}_{\mathbf{h}}\right)$, o Método de Expansões Sucessivas exige apenas $\left|\mathbb{S}_{\mathbf{h}}\right|$ iterações em vez de $|\mathbb{L}|-\left|\mathbb{S}_{\mathbf{h}}\right|$ iterações exigidas pelo método de Encolhimentos Sucessivos. Portanto, o Método de Expansões Sucessivas é computacionalmente mais vantajoso que o Método de Encolhimentos Sucessivos quando $\left|\mathbb{S}_{\mathbf{h}}\right| \leq(|\mathbb{L}| \div 2)$. 


\subsection{4}

\section{Análise de desempenho}

As Figuras 4.15, 4.16, 4.17 e 4.18 mostram o desempenho do método de Expansões Sucessivas, com $\lambda=1,05$, nas versões sem e com despolarização, comparado com o desempenho das estimações LS full-support e LS Oráculo, em função do comprimento $K$ da sequência de treinamento, avaliado sobre $Q=1000$ realizações independentes de um sistema $\operatorname{MESS}(100,10)$, com N=16 e SINR=5, 10, 15, 20, 25 e 30 dB respectivamente. Nos gráficos superiores, são mostrados os desempenhos dos algoritmos citados em termos de NMSD. Nos gráficos inferiores, são mostradas as probabilidades de ocorrência dos possíveis eventos de encolhimento. As Figuras 4.21, 4.22, 4.23 e 4.24 mostram o desempenho dos algoritmos citados para a estimação de um sistema $\operatorname{MESS}(100,20)$, mantendo-se inalterados os demais parâmetros de simulação. As figuras mostram que, quanto maior SINR ou menor $S$, menores as probabilidades de ocorrências de sub-encolhimentos e encolhimentos disformes, aumentando-se assim o desempenho do algoritmo proposto.
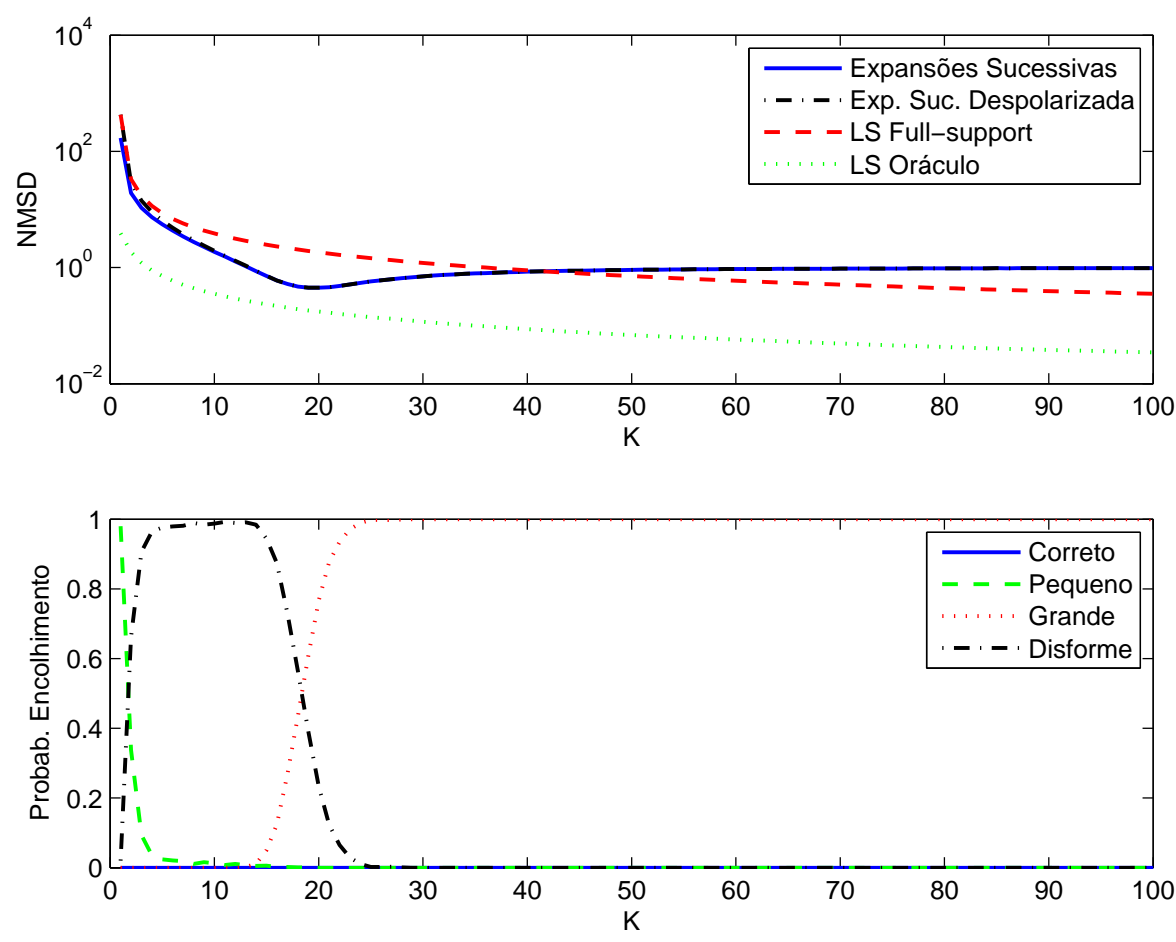

Figura 4.15: Exp. Suc.: $\operatorname{MESS}(100,10), \mathrm{SINR}=5 \mathrm{~dB}, \lambda=1,05, \mathrm{~N}=16$ e $\mathrm{Q}=1000$ 

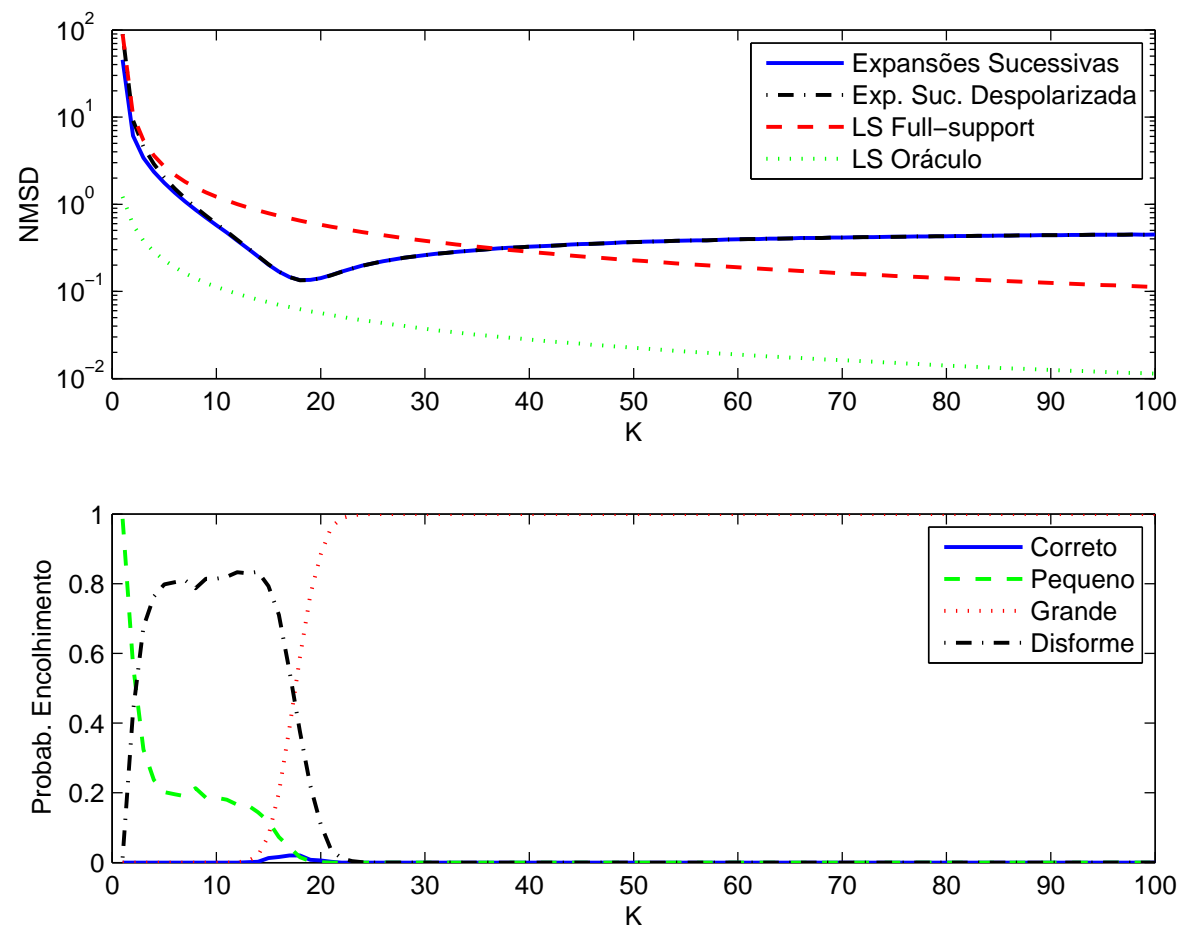

Figura 4.16: Exp. Suc.: $\operatorname{MESS}(100,10), \operatorname{SINR}=10 d B, \lambda=1,05, \mathrm{~N}=16$ e $\mathrm{Q}=1000$
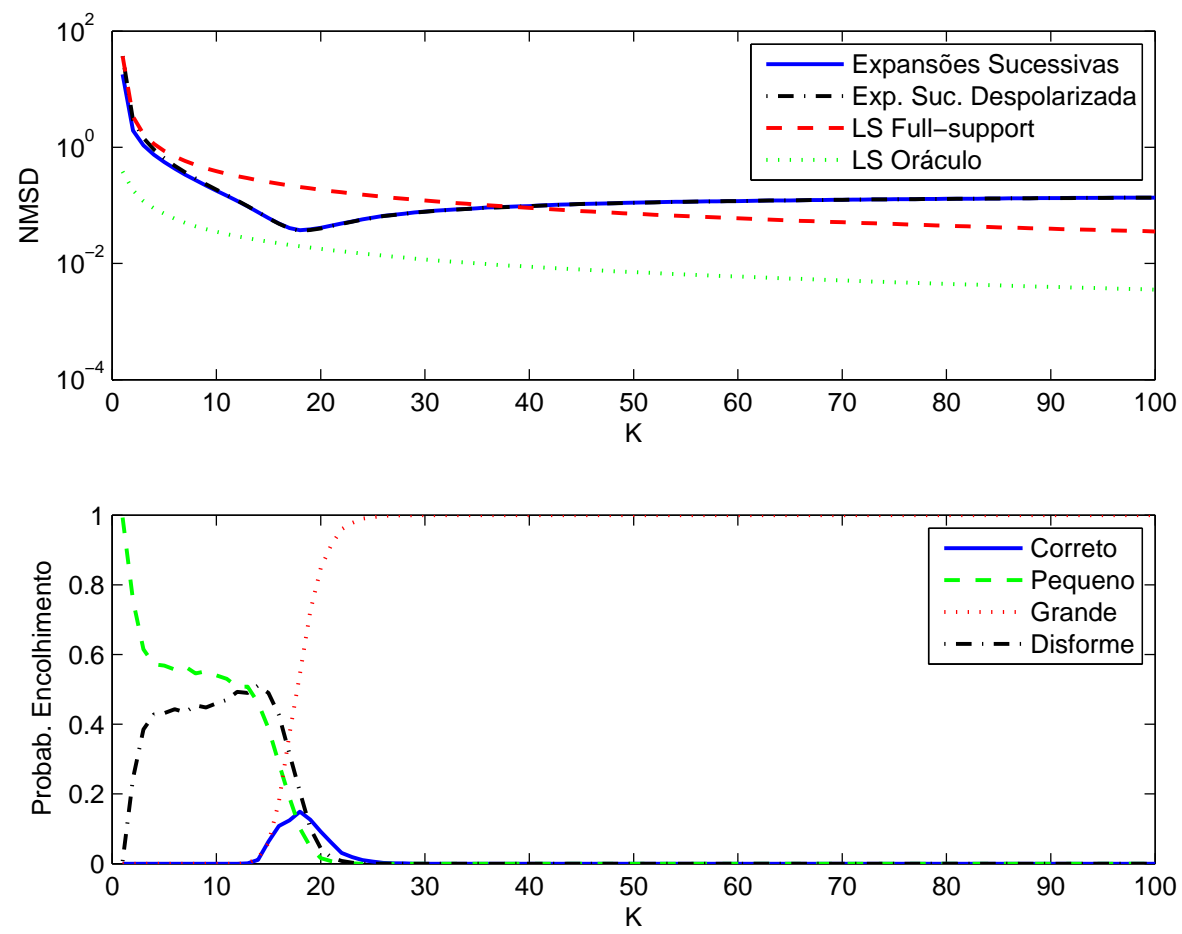

Figura 4.17: Exp. Suc.: MESS(100,10), SINR=15dB, $\lambda=1,05, N=16$ e Q=1000 

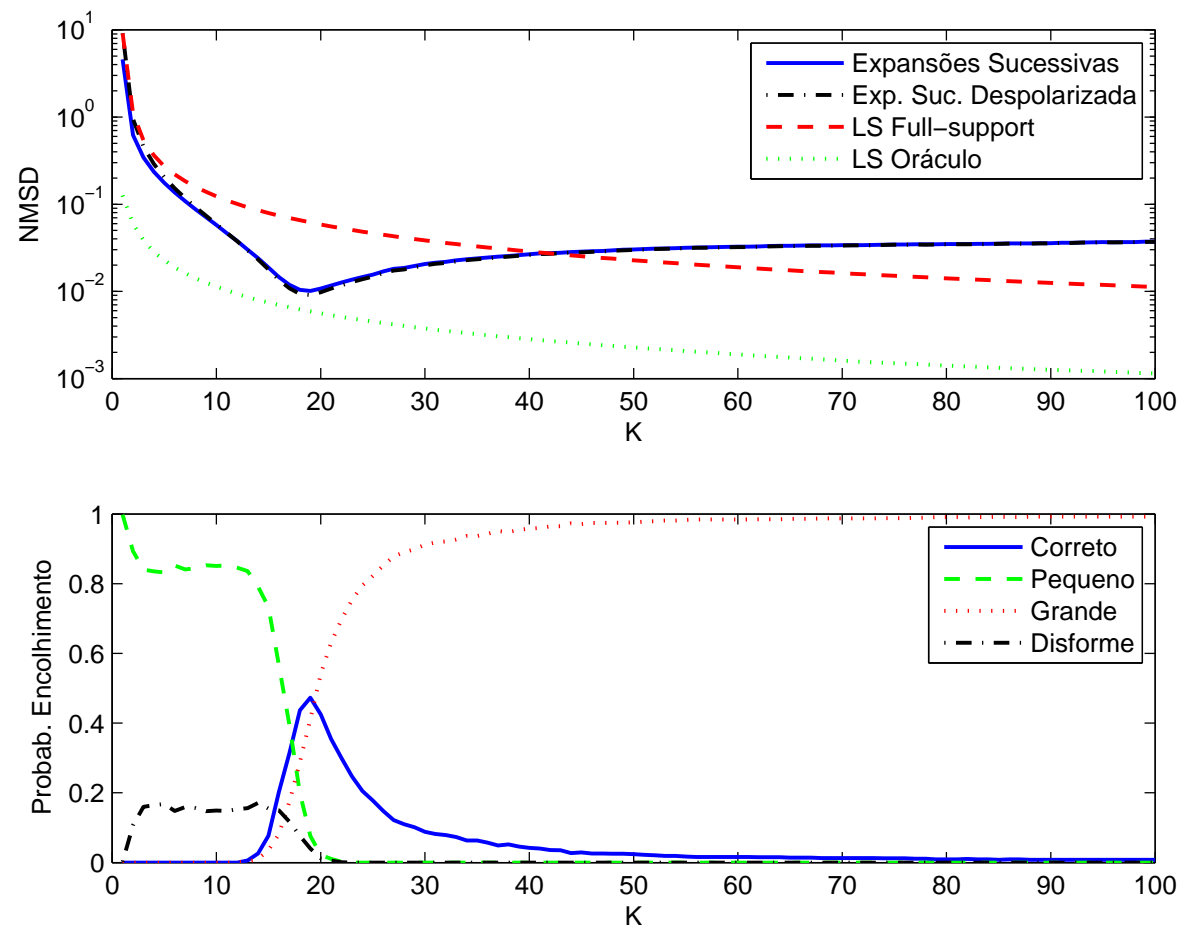

Figura 4.18: Exp. Suc.: $\operatorname{MESS}(100,10), \operatorname{SINR}=20 d B, \lambda=1,05, N=16$ e $Q=1000$
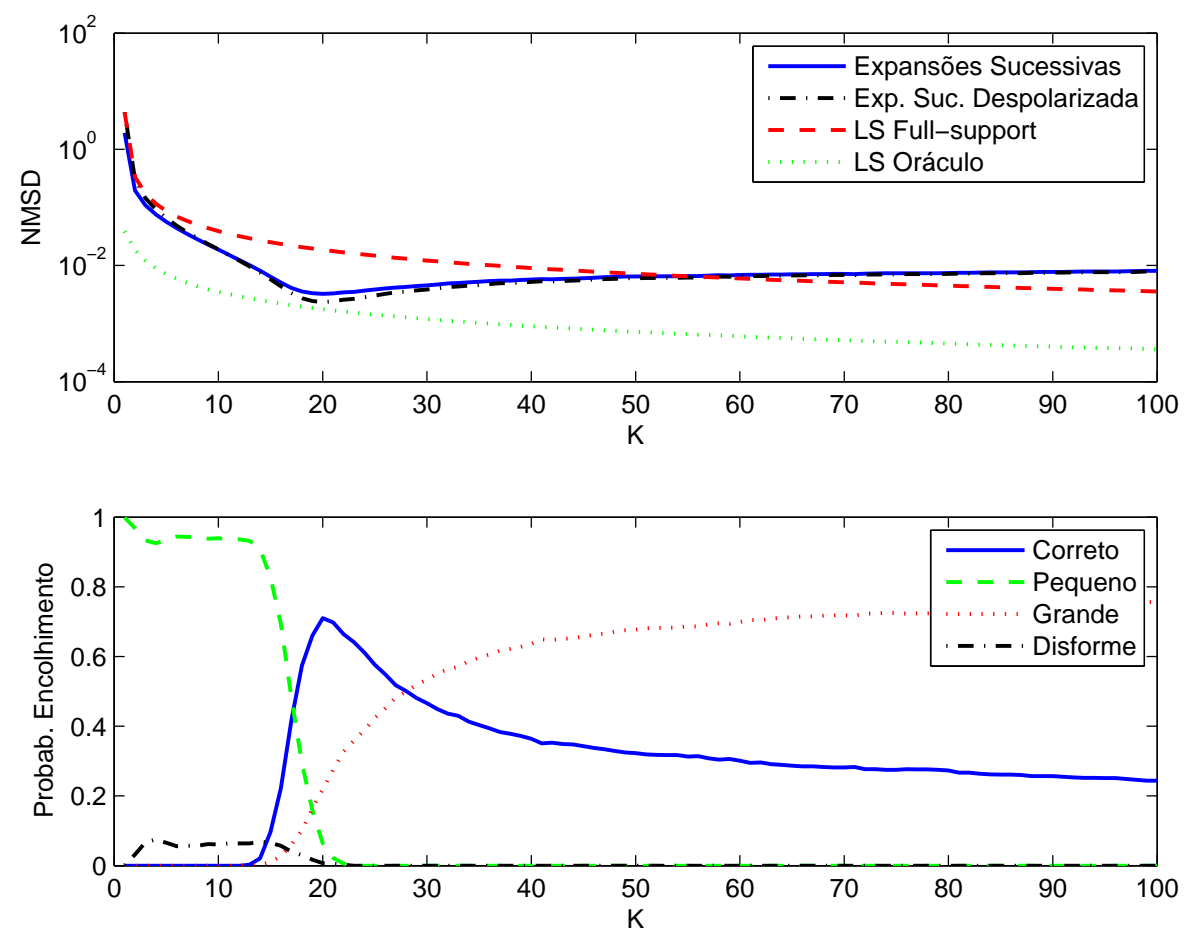

Figura 4.19: Exp. Suc.: $\operatorname{MESS}(100,10), \mathrm{SINR}=25 \mathrm{~dB}, \lambda=1,05, \mathrm{~N}=16$ e $\mathrm{Q}=1000$ 

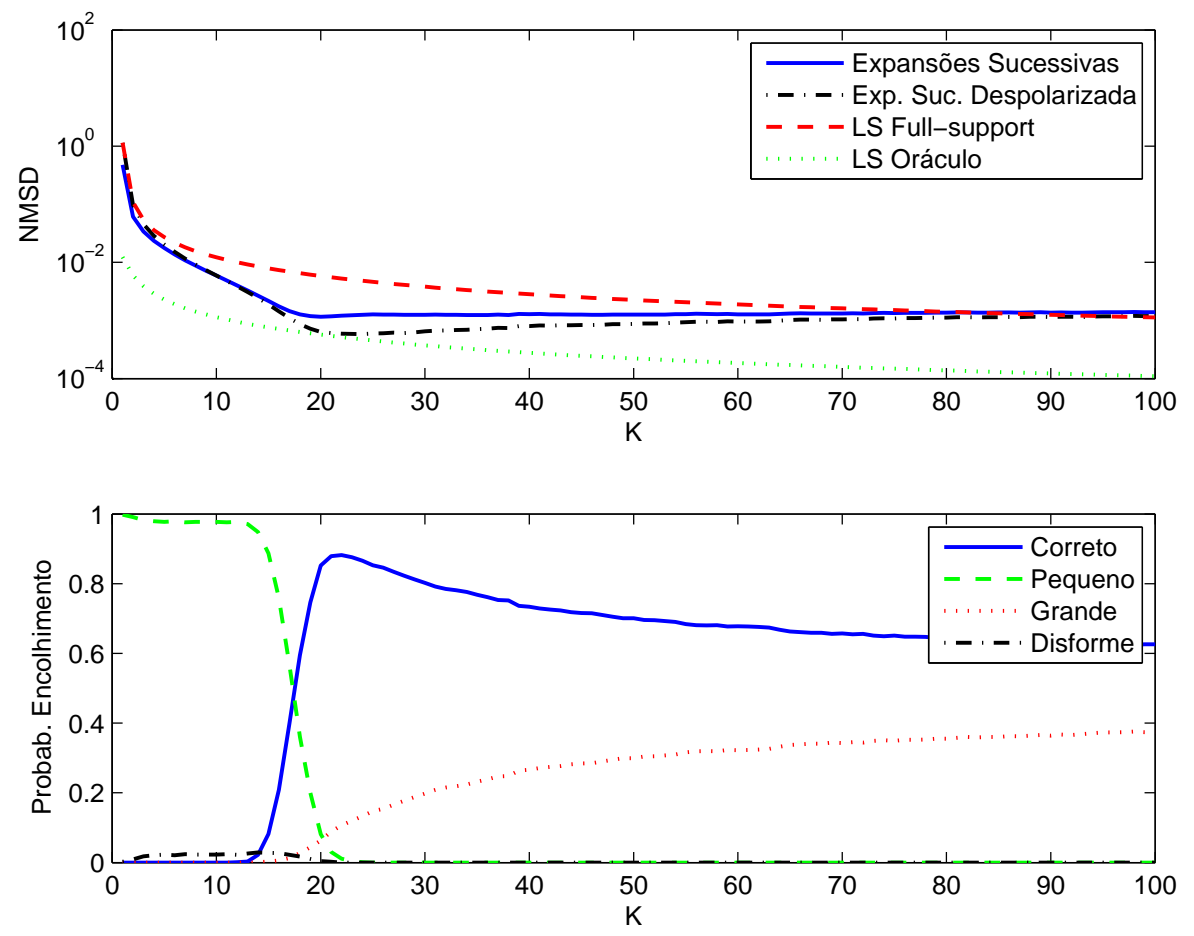

Figura 4.20: Exp. Suc.: $\operatorname{MESS}(100,10), \operatorname{SINR}=30 d B, \lambda=1,05, N=16$ e $Q=1000$
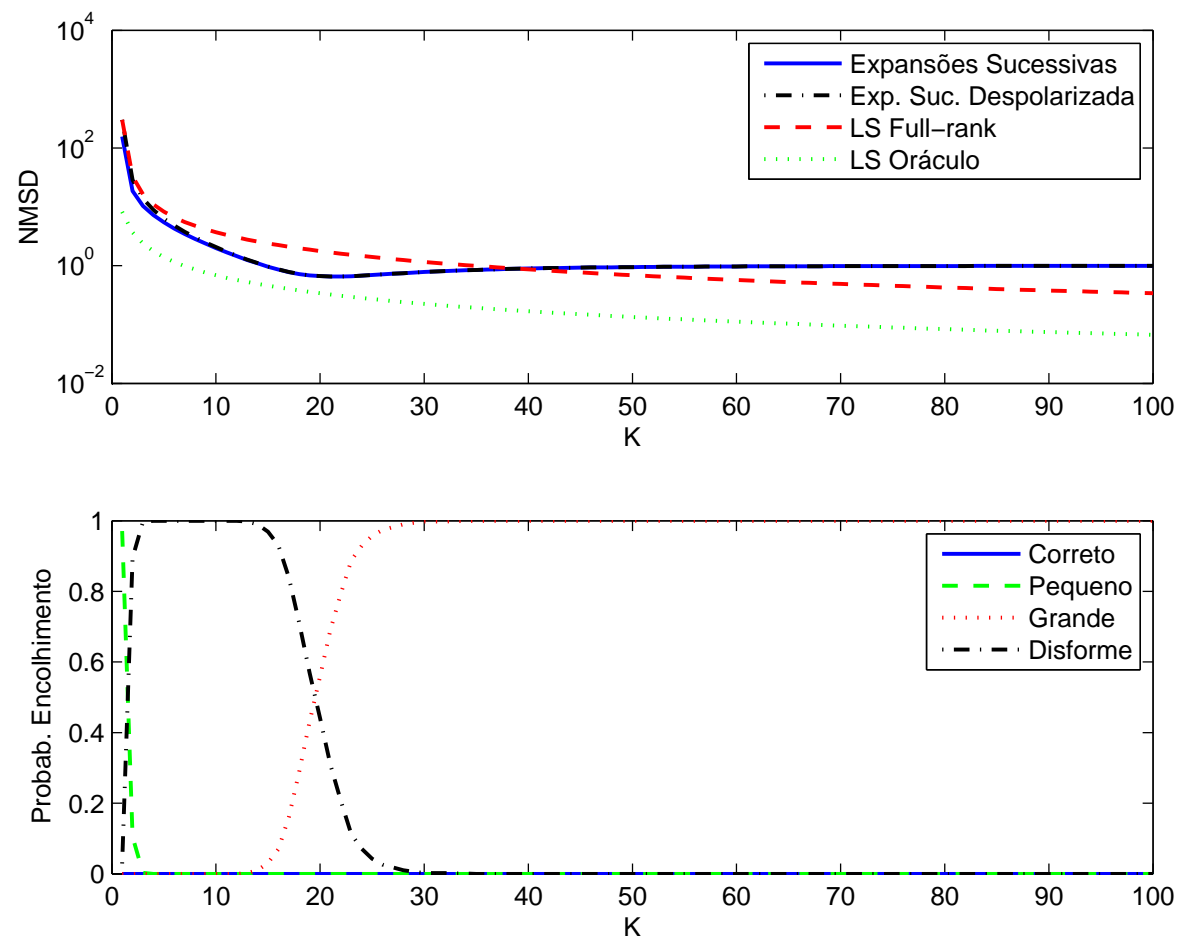

Figura 4.21: Exp. Suc.: $\operatorname{MESS}(100,20), \operatorname{SINR}=5 \mathrm{~dB}, \lambda=1,05, \mathrm{~N}=16$ e $\mathrm{Q}=1000$ 

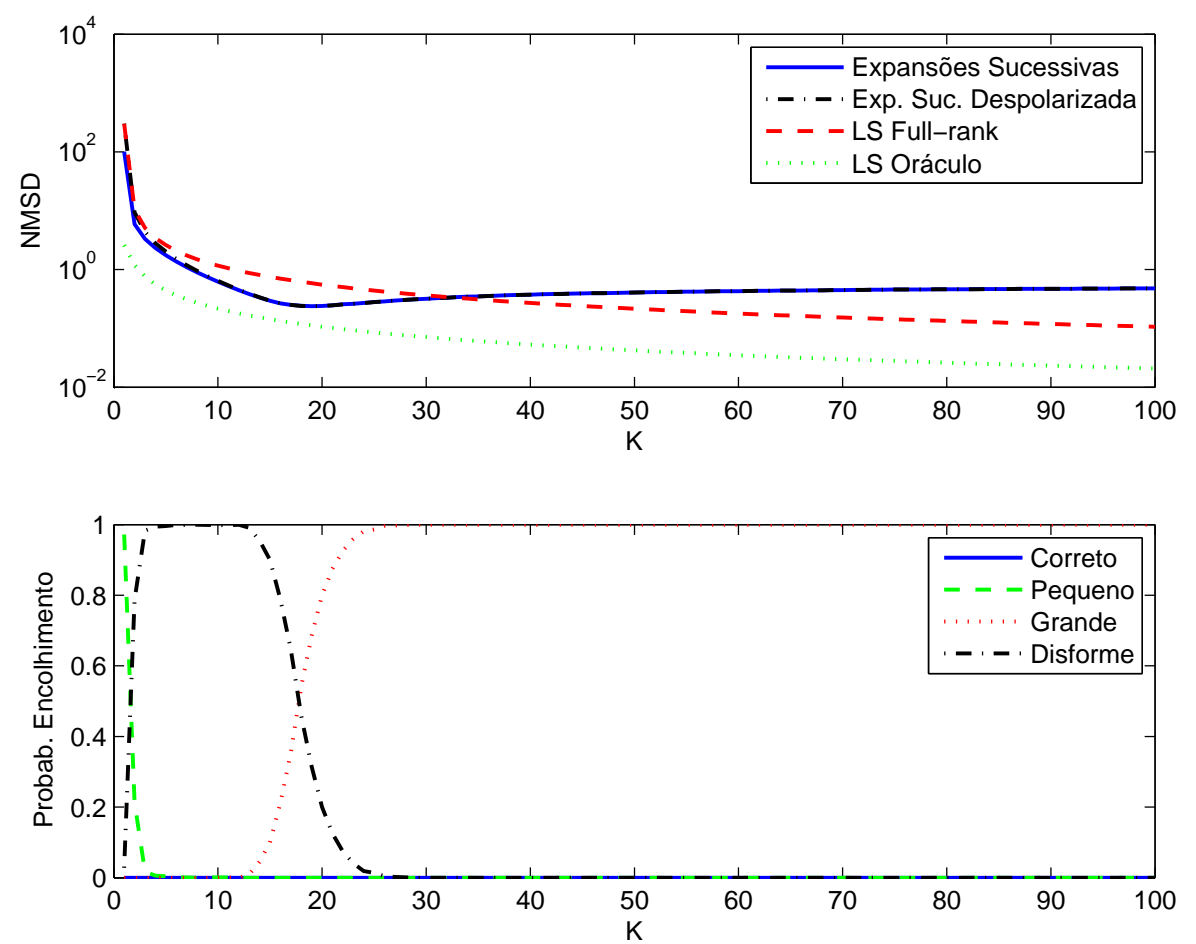

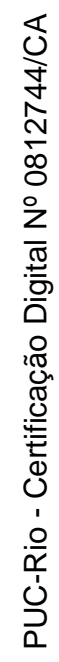

Figura 4.22: Exp. Suc.: $\operatorname{MESS}(100,20), \operatorname{SINR}=10 d B, \lambda=1,05, N=16$ e Q=1000
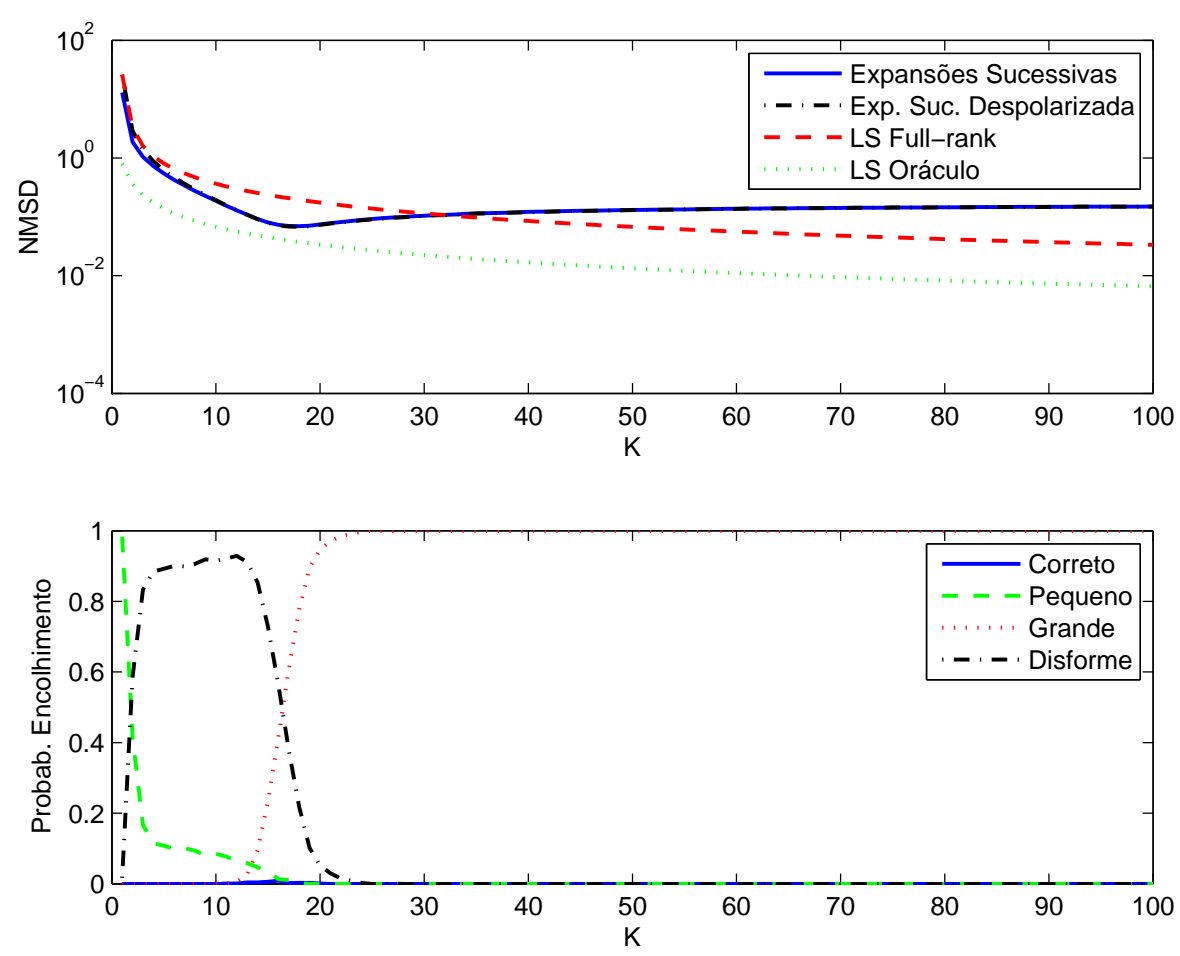

Figura 4.23: Exp. Suc.: $\operatorname{MESS}(100,20), \operatorname{SINR}=15 d B, \lambda=1,05, \mathrm{~N}=16$ e $\mathrm{Q}=1000$ 

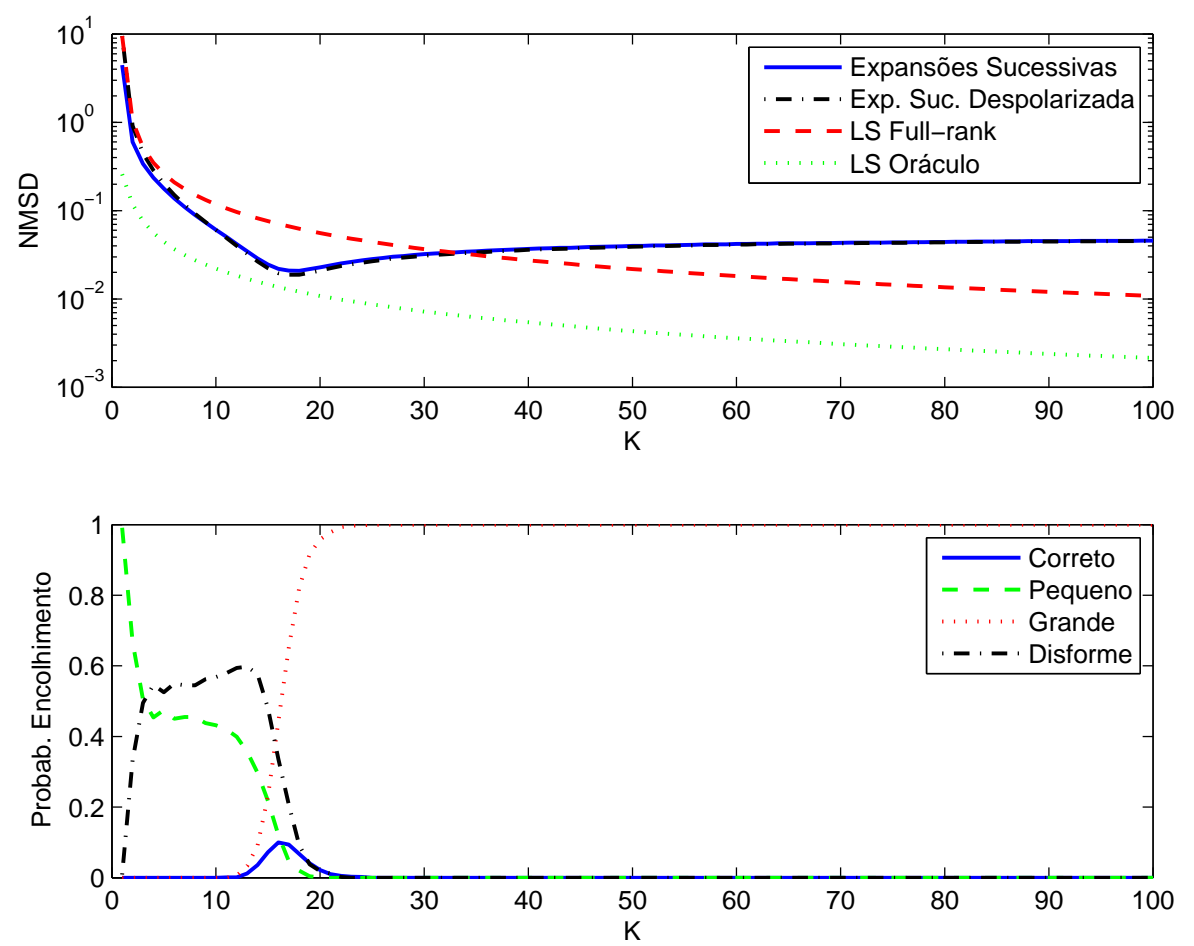

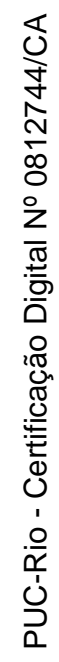

Figura 4.24: Exp. Suc.: $\operatorname{MESS}(100,20), \operatorname{SINR}=20 d B, \lambda=1,05, N=16$ e $Q=1000$
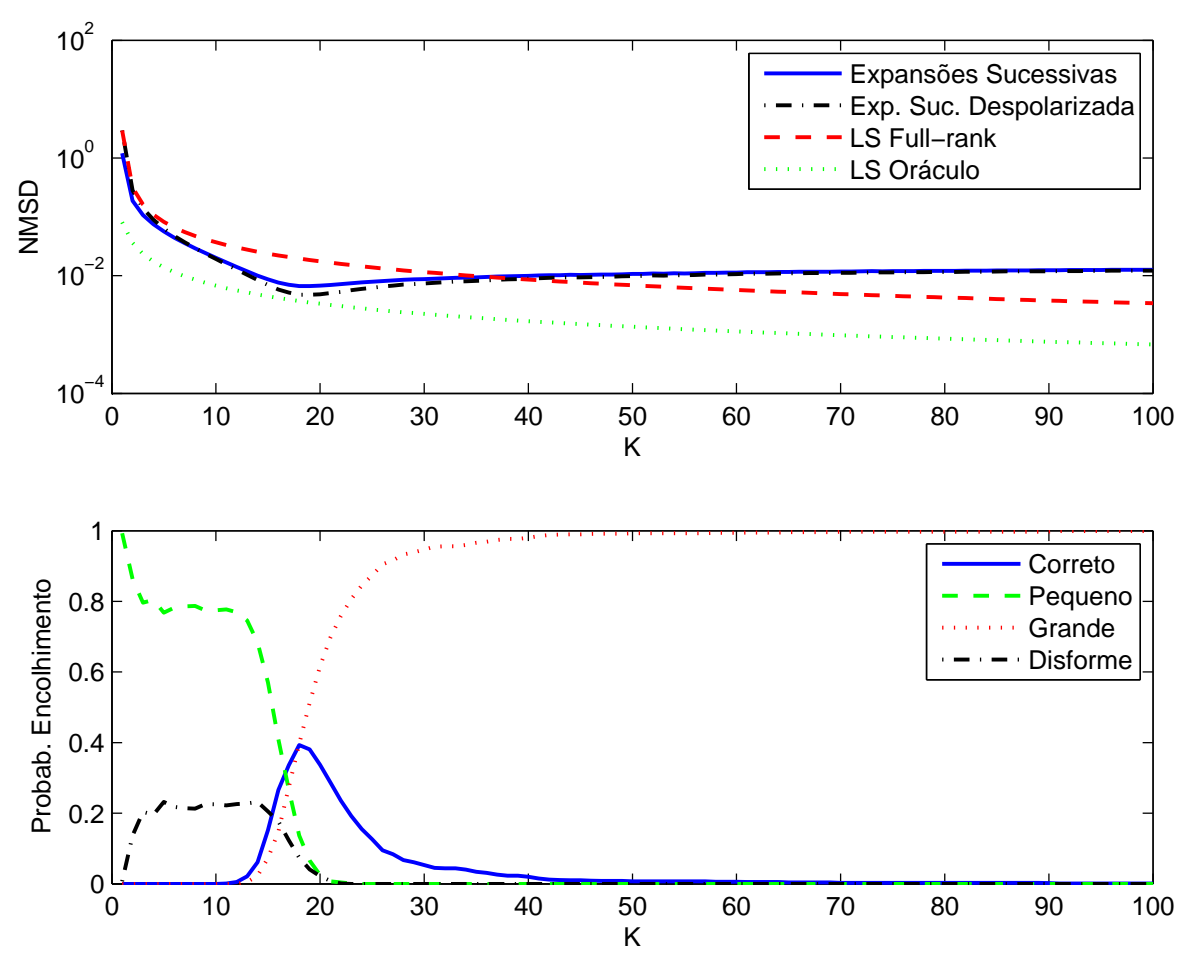

Figura 4.25: Exp. Suc.: $\operatorname{MESS}(100,20), \operatorname{SINR}=25 \mathrm{~dB}, \lambda=1,05, \mathrm{~N}=16$ e $\mathrm{Q}=1000$ 

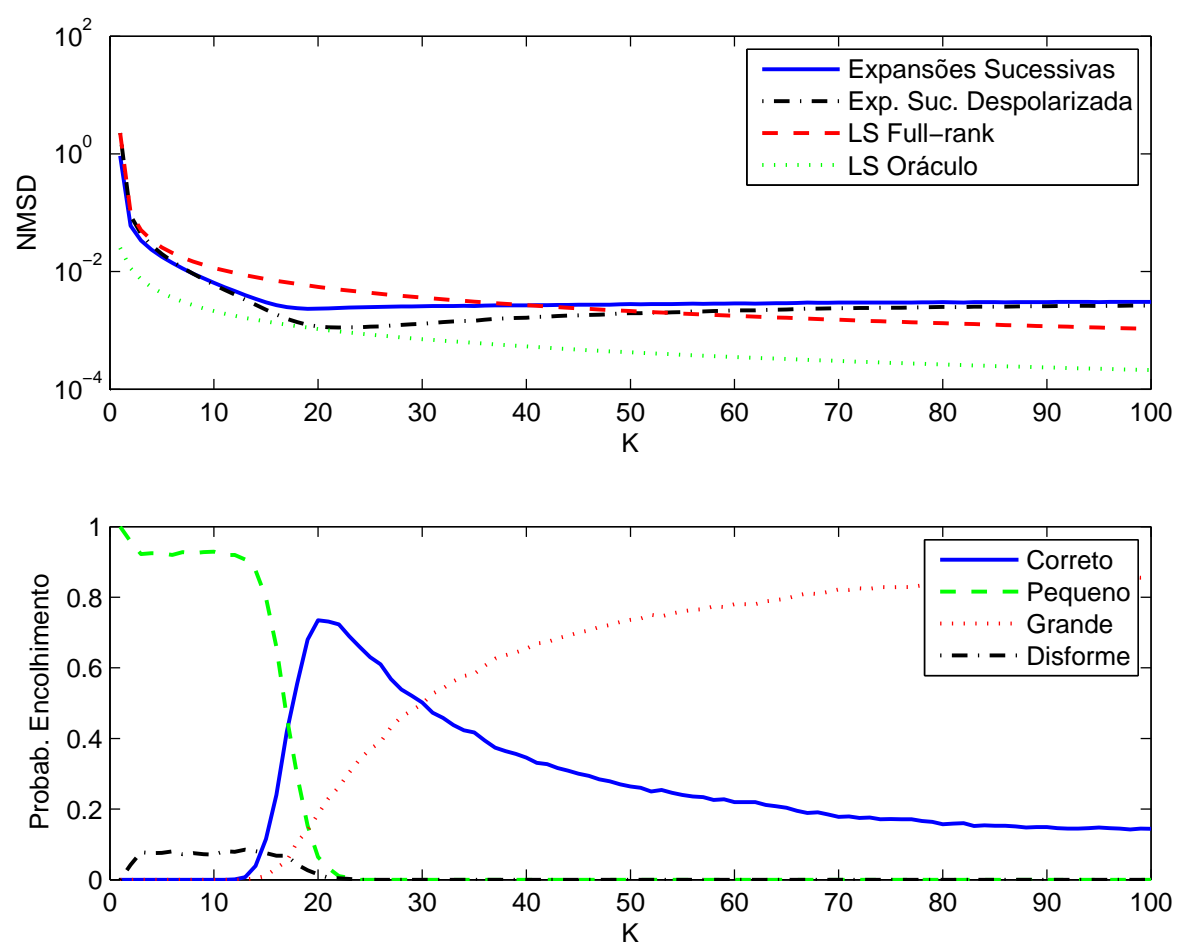

Figura 4.26: Exp. Suc.: $\operatorname{MESS}(100,20), \operatorname{SINR}=30 d B, \lambda=1,05, N=16$ e $Q=1000$

\section{7}

\section{Método de Minimização da Norma L1}

\subsection{1}

\section{Introdução}

Da Teoria de Compressive Sensing, origina-se a técnica de estimação denominada Basis Pursuit, cujo critério de otimização é dado por (13)

$$
\tilde{\mathbf{x}}=\arg \min _{\mathbf{x}}\|\mathbf{x}\|_{1} \text { tal que } \mathbf{H x}=\mathbf{r}
$$

onde $\mathbf{r}$ é o vetor que representa a observação e $\mathbf{H}$ uma matriz de dimensão $m \times n$, cujas colunas formam uma base sobredeterminada (ou seja, $m \leq n$ ).

A formulação da técnica de Basis Pursuit, adaptada para observações com ruído, é

$$
\tilde{\mathbf{x}}=\arg \min _{\mathbf{x}} J_{k}(\mathbf{x})+\gamma\|\mathbf{x}\|_{1}
$$

onde $\gamma$ é um fator de regulação arbitrado. Quanto maior o valor de $\gamma$, mais esparso $\tilde{\mathbf{x}}$. 
O operador LASSO (12), que tem forte conexão com a técnica de Basis Pursuit, pode ser formulado da seguinte forma

$$
\tilde{\mathbf{x}}=\arg \min _{\mathbf{x}} J_{k}(\mathbf{x}) \text { tal que }\|\mathbf{x}\|_{1} \leq t
$$

onde $t$ é um parâmetro que limita o grau de esparsidade de $\tilde{\mathbf{x}}$.

\subsection{2}

\section{Algoritmo proposto}

Inspirado nas técnicas de Basis Pursuit e LASSO, o método de Minimização da Norma $l_{1}$, aqui proposto, consiste em encontrar a estimativa $\tilde{\mathbf{h}}_{k}^{\mathbb{L}}$, associada ao suporte $\mathbb{L}$, no instante $k$, dada por

$$
\tilde{\mathbf{h}}_{k}^{\mathbb{L}}=\arg \min _{\mathbf{x}}\|\mathbf{x}\|_{1} \text { tal que } J_{k}^{\mathbb{L}}(\mathbf{x})<J_{\max }
$$

onde $J_{k}^{\mathbb{L}}(\mathbf{x})$ é o custo LS, dado por 3-6, e $J_{\max }$ é o valor máximo admitido para o custo LS.

Se $J_{k}^{\mathbb{L}}(\mathbf{0})<J_{\max }$, então 4-37 tem como solução única $\tilde{\mathbf{h}}_{k}^{\mathbb{L}}=\mathbf{0}$. Caso contrário, como $\|\cdot\|_{1}$ é função convexa e $J_{k}^{\mathbb{L}}($.) é estritamente convexa, então 4-37 possui uma solução única não-trivial $\tilde{\mathbf{h}}_{k}^{\mathbb{L}} \neq \mathbf{0}$.

O problema enunciado em 4-37 é equivalente a

$$
\tilde{\mathbf{h}}_{k}^{\mathbb{L}}=\arg \min _{\mathbf{x}}\|\mathbf{x}\|_{1}+\phi\left[J_{k}^{\mathbb{L}}(\mathbf{x})-J_{\max }\right]
$$

em que $\phi(z): \mathbb{R} \rightarrow \mathbb{R}$ é a função barreira ideal, definida como

$$
\phi(z)=\left\{\begin{array}{cc}
0 & z<0 \\
\infty & z \geq 0
\end{array}\right.
$$

Entretanto, $\phi(z)$ é não-diferenciável. Baseado em técnicas de ponto interior (28), a solução para 4-38 pode ser obtida analiticamente e corresponde à solução, quando $u$ tende a infinito, para a seguinte expressão

$$
\tilde{\mathbf{h}}_{k}^{\mathbb{L}}=\arg \min _{\mathbf{x}} f_{u}(\mathbf{x})
$$

onde

$$
f_{u}(\mathbf{x})=\|\mathbf{x}\|_{1}+\phi_{u}\left[J_{k}^{\mathbb{L}}(\mathbf{x})-J_{\max }\right]
$$

e $\phi_{u}(z): \mathbb{R} \rightarrow \mathbb{R}$ é a função barreira logarítmica (com parâmetro $u \in \mathbb{R}^{+}$), definida como 


$$
\phi_{u}(z)=\left\{\begin{array}{cc}
-\frac{1}{2 u} \log (-z) & z<0 \\
\infty & z \geq 0
\end{array}\right.
$$

A função $J_{k}^{\mathbb{L}}()-.J_{\max }$ é convexa. A função $\phi_{u}($.$) , além de convexa, é crescente,$ para todo $u \in \mathbb{R}^{+}$. Logo, a composição $\phi_{u}\left[J_{k}^{\mathbb{L}}()-.J_{\max }\right]$ é convexa. Como \|\|$_{1}$ é convexa, então $f_{u}($.$) também é convexa e, portanto, possui um único ponto$ de mínimo global. À medida em que $u$ tende a infinito, a função barreira logarítmica converge para a função barreira ideal e, consequentemente, a solução para 4-40 converge para a solução ao problema originalmente enunciado em 4-37.

O desenvolvimento do método proposto, apresentado a seguir, é desmembrado em duas hipóteses:

- $\tilde{\mathbf{h}}_{k}^{\mathbb{L}}$ não possui nenhum elemento nulo;

- $\tilde{\mathbf{h}}_{k}^{\mathbb{L}}$ possui um ou mais elementos nulos.

\section{Primeira hipótese: $\tilde{\mathbf{h}}_{k}^{\mathbb{L}}$ não possui nenhum elemento nulo}

Seja, por hipótese, $\mathbf{x}=\left[x_{1} x_{2} \ldots x_{|\mathbb{L}|}\right]^{T}$ um vetor de dimensão $|\mathbb{L}| \times 1$ com todos os seus elementos diferentes de zero. Nesse caso, o gradiente de $f_{u}(\mathbf{x})$ é dado por

$$
\nabla f_{u}(\mathbf{x})=\operatorname{sgn}(\mathbf{x})+u^{-1}\left[J_{\max }-J_{k}^{\mathbb{L}}(\mathbf{x})\right]^{-1}\left[\left(\mathbf{A}_{k}\right)_{\mathbb{S}, \mathbb{S}} \mathbf{x}-\left(\mathbf{b}_{k}\right)_{\mathbb{S}}\right]
$$

onde $\operatorname{sgn}(\mathbf{x})=\left[\operatorname{sgn}\left(x_{1}\right) \operatorname{sgn}\left(x_{2}\right) \ldots \operatorname{sgn}\left(x_{N}\right)\right]^{T}$, para um vetor-coluna de comprimento N. A função $\operatorname{sgn}(z): \mathbb{C}^{*} \rightarrow \mathbb{C}$ é dada por

$$
\operatorname{sgn}(z)=\frac{z}{|z|} \quad \forall z \neq 0
$$

A solução analítica de 4-40 é encontrada fazendo $\nabla f_{u}\left(\tilde{\mathbf{h}}_{k}^{\mathbb{L}}\right)=\mathbf{0}$. Nesse caso, tem-se que

$$
\operatorname{sgn}\left(\tilde{\mathbf{h}}_{k}^{\mathbb{L}}\right)+\delta_{u}^{-1}\left[\left(\mathbf{A}_{k}\right)_{\mathbb{S}, \mathbb{S}} \tilde{\mathbf{h}}_{k}^{\mathbb{L}}-\left(\mathbf{b}_{k}\right)_{\mathbb{S}}\right]=\mathbf{0}
$$

em que

$$
\delta_{u}=u\left[J_{\max }-J_{k}^{\mathbb{L}}\left(\tilde{\mathbf{h}}_{k}^{\mathbb{L}}\right)\right]
$$

Seja $\mathbf{M}_{\mathbf{x}}$ a matriz-diagonal formada pelos módulos dos elementos de $\mathbf{x}$, ou seja 


$$
M_{\mathbf{x}}=\left[\begin{array}{cccc}
\left|x_{1}\right| & & & \\
& \left|x_{2}\right| & & \\
& & \ddots & \\
& & & \left|x_{|\mathbb{L}|}\right|
\end{array}\right]
$$

Dessa forma, tem-se que

$$
\operatorname{sgn}(\mathbf{x})=\mathbf{M}_{\mathbf{x}}^{-1} \mathbf{x}
$$

Utilizando 3-9 e 4-48, 4-45 pode ser reescrita como

$$
\left\{\mathbf{I}+\delta_{u}\left(\mathbf{A}_{k}\right)_{\mathbb{S}, \mathbb{S}}^{-1} \mathbf{M}_{\tilde{\mathbf{h}}_{k}^{\mathbb{L}}}^{-1}\right\} \tilde{\mathbf{h}}_{k}^{\mathbb{L}}=\hat{\mathbf{h}}_{k}^{\mathbb{L}}
$$

Seja, por hipótese, $\left(\mathbf{A}_{k}\right)_{\mathbb{S}, \mathbb{S}}=\mathbf{I}$ (condição atendida quando $k=\infty$ ). Nesse caso, a equação 4-49 pode ser simplificada da seguinte forma

$$
\mathbf{D} \tilde{\mathbf{h}}_{k}^{\mathbb{L}}=\hat{\mathbf{h}}_{k}^{\mathbb{L}}
$$

onde D é uma matriz-diagonal dada por

$$
\mathbf{D}=\mathbf{I}+\delta_{u} \mathbf{M}_{\tilde{\mathbf{h}}_{k}^{\mathbb{L}}}^{-1}
$$

Portanto, a solução $\tilde{\mathbf{h}}_{k}^{\mathbb{L}}$ ao problema enunciado em 4-40, quando $k$ tende a infinito, pode ser expressa como

$$
\tilde{\mathbf{h}}_{k}^{\mathbb{L}}=\mathbf{D}^{-1} \hat{\mathbf{h}}_{k}^{\mathbb{L}}
$$

A equação 4-50 pode ser expressa como

$$
d_{i}\left(\tilde{\mathbf{h}}_{k}^{\mathbb{L}}\right)_{i}=\left(\hat{\mathbf{h}}_{k}^{\mathbb{L}}\right)_{i} \quad i=1,2, \ldots,|\mathbb{L}|
$$

em que

$$
d_{i}=1+\frac{\delta_{u}}{\left|\left(\tilde{\mathbf{h}}_{k}^{\mathbb{L}}\right)_{i}\right|} \quad \forall i
$$

Como $u \in \mathbb{R}^{+}$e, por hipótese, $J_{k}^{\mathbb{L}}\left(\tilde{\mathbf{h}}_{k}^{\mathbb{L}}\right)<J_{\max }$, então $\delta_{u}>0$ para todo $u$ e, consequentemente, $d_{i}>1$ para todo $i$. Logo, ao se aplicar o operador módulo nos dois lados da equação 4-53, tem-se que

$$
\left[1+\frac{\delta_{u}}{\left|\left(\tilde{\mathbf{h}}_{k}^{\mathbb{L}}\right)_{i}\right|}\right]\left|\left(\tilde{\mathbf{h}}_{k}^{\mathbb{L}}\right)_{i}\right|=\left|\left(\hat{\mathbf{h}}_{k}^{\mathbb{L}}\right)_{i}\right|
$$

que pode ser rearranjada da seguinte forma 


$$
\left|\left(\tilde{\mathbf{h}}_{k}^{\mathbb{L}}\right)_{i}\right|=\left|\left(\hat{\mathbf{h}}_{k}^{\mathbb{L}}\right)_{i}\right|-\delta_{u} \quad \forall i
$$

Como, por hipótese, $\left(\tilde{\mathbf{h}}_{k}^{\mathbb{L}}\right)_{i} \neq 0$ para todo $i$, então 4-56 implica em

$$
\delta_{u}<\min _{i}\left\{\left|\left(\hat{\mathbf{h}}_{k}^{\mathbb{L}}\right)_{i}\right|\right\} \quad \forall u
$$

A equação 4-56 mostra que o método proposto provoca um encolhimento de $\hat{\mathbf{h}}_{k}^{\mathbb{L}}$ de tal forma que todos seus elementos sofrem uma mesma redução $\delta_{u}$ em seus respectivos módulos. Uma solução ao problema enunciado em 4-40 que atende à condição estabelecida em 4-56 é dada por

$$
\left(\tilde{\mathbf{h}}_{k}^{\mathbb{L}}\right)_{i}=\left[1-\frac{\delta_{u}}{\left|\left(\hat{\mathbf{h}}_{k}^{\mathbb{L}}\right)_{i}\right|}\right]\left(\hat{\mathbf{h}}_{k}^{\mathbb{L}}\right)_{i}
$$

Lembrando que, para qualquer valor de $u$, existe uma única solução para 4-40, haja vista que $f_{u}($.$) é convexa, então conclui-se que a solução dada por 4-58 é$ a única solução para 4-40.

Alternativamente, a solução para a equação 4-40 pode ser determinada da seguinte forma. Seja $\Delta \mathbf{h}=\tilde{\mathbf{h}}_{k}^{\mathbb{L}}-\hat{\mathbf{h}}_{k}^{\mathbb{L}}$. Como, por hipótese, $\mathbf{A}_{k}=\mathbf{I}$ então, empregando 4-16, tem-se que

$$
J_{k}^{\mathbb{L}}\left(\tilde{\mathbf{h}}_{k}^{\mathbb{L}}\right)=J_{k}(\mathbb{L})+\|\Delta \mathbf{h}\|^{2}
$$

Além disso, observando 4-53 e lembrando que $d_{i}>1$ para todo $i$, então é possível afirmar que

$$
\left\|\tilde{\mathbf{h}}_{k}^{\mathbb{L}}\right\|_{1}=\left\|\hat{\mathbf{h}}_{k}^{\mathbb{L}}\right\|_{1}-\|\Delta \mathbf{h}\|_{1}
$$

Portanto, minimizar $\left\|\tilde{\mathbf{h}}_{k}^{\mathbb{L}}\right\|_{1}$ corresponde a maximizar $\|\Delta \mathbf{h}\|_{1}$, tal que a condição estabelecida em 4-59 seja atendida. Dado o valor de $\|\Delta \mathbf{h}\|_{2}^{2}$ (ou equivalentemente, o valor de $J_{k}^{\mathbb{L}}\left(\tilde{\mathbf{h}}_{k}^{\mathbb{L}}\right)$ ), o Teorema 4.3 demonstra que $\|\Delta \mathbf{h}\|_{1}$ é maximizada quando $\Delta \mathbf{h}=\delta_{u} \mathbf{1}$, o que vai ao encontro da condição estabelecida em 4-56. Logo, é possível afirmar que

$$
\delta_{u}=\sqrt{\frac{\|\Delta \mathbf{h}\|_{2}^{2}}{|\mathbb{L}|}}
$$

Empregando 4-59 em 4-61, tem-se que

$$
\delta_{u}=\sqrt{\frac{J_{k}^{\mathbb{L}}\left(\tilde{\mathbf{h}}_{k}^{\mathbb{L}}\right)-J_{k}(\mathbb{L})}{|\mathbb{L}|}}
$$


Combinando 4-46 e 4-62, tem-se que

$$
\delta_{u}=u\left[J_{\max }-J_{k}(\mathbb{L})-|\mathbb{L}| \delta_{u}^{2}\right]
$$

A equação 4-63 corresponde a um polinômio de segundo grau em $\delta_{u}$, cuja única raiz real não-negativa é dada por

$$
\delta_{u}=\frac{\sqrt{u^{-2}+4|\mathbb{L}|\left[J_{\max }-J_{k}(\mathbb{L})\right]}-u^{-1}}{2|\mathbb{L}|}
$$

Portanto, dados $J_{\max }$ e $u$, é possível obter o valor correspondente de $\delta_{u}$ através de 4-64. Se o valor obtido atender à condição estabelecida em 4-57, então quando $k$ tende a infinito, $\tilde{\mathbf{h}}_{k}^{\mathbb{L}}$ obtido através de $4-58$ obedece à hipótese de que todos seus elementos são diferentes de zero e corresponde à única solução de 4-40.

\section{Segunda hipótese: $\tilde{\mathbf{h}}_{k}^{\mathbb{L}}$ possui um ou mais elementos nulos}

Se o valor de $\delta_{u}$, obtido através de 4-64, não atender à condição estabelecida em 4-57, então como a solução de 4-40 existe e é única, conclui-se que $\tilde{\mathbf{h}}_{k}^{\mathbb{L}}$ possui um ou mais elementos nulos.

Considere as seguintes variáveis

$$
\begin{gathered}
l=\arg \min _{j}\left\{\left|\left(\hat{\mathbf{h}}_{k}^{\mathbb{S}^{(i)}}\right)_{j}\right|\right\} \\
\mathbb{S}^{(i+1)}=\mathbb{S}^{(i)} \backslash\{l\} \\
a^{(i)}=\left|\left(\hat{\mathbf{h}}_{k}^{\mathbb{S}^{(i)}}\right)_{l}\right|
\end{gathered}
$$

Sejam $\mathbb{V}^{(i)}$ o sub-espaço subjacente a $\mathbb{S}^{(i)}, \mathbf{x} \in \mathbb{V}^{(i)}$ o vetor obtido através de 458 (substituindo $\mathbb{L}$ por $\mathbb{S}^{(i)}$ ) para o caso particular em que $\delta_{u}=a^{(i-1)}$ e $\mathbf{y} \in \mathbb{V}^{(i)}$ o vetor obtido através de 4-58 para $\delta_{u}=b$, com $a^{(i-1)}<b<a^{(i)}$. À medida em que o valor de $\delta_{u}$ varia de 0 até $b$, descreve-se uma curva em $\mathbb{V}^{(i)}$, iniciando em $\hat{\mathbf{h}}_{k}^{\mathbb{S}(i)}$, passando por $\mathbf{x}$ e terminando em $\mathbf{y}$. A distância $\left\|\mathbf{x}-\hat{\mathbf{h}}_{k}^{\mathbb{S}^{(i)}}\right\|_{1}$ é a máxima distância correspondente à variação de $\delta_{u}$ de 0 até $a^{(i-1)}$. A distância $\left\|\mathbf{y}-\hat{\mathbf{h}}_{k}^{\mathbb{S}^{(i)}}\right\|_{1}$ é a máxima distância correspondente à variação de $\delta_{u}$ de 0 até b. Logo, devido à propriedade triangular das normas, é possível afirmar que a distância $\|\mathbf{y}-\mathbf{x}\|_{1}$ é a máxima distância correspondente à variação de $\delta_{u}$ de $a^{(i-1)}$ até $b$, dentro do sub-espaço $\mathbb{V}^{(i)}$.

Seja $\mathbf{z} \in \mathbb{V}^{(i-1)}$ o vetor obtido através de 4-58 (substituindo $\mathbb{L}$ por $\mathbb{S}^{(i-1)}$ ) para o caso particular em que $\delta_{u}=a^{(i-1)}$. A distância $\left\|\mathbf{z}-\hat{\mathbf{h}}_{k}^{\mathbb{S}^{(i-1)}}\right\|_{1}$ é a máxima distância correspondente à variação de $\delta_{u}$ de 0 até $a^{(i-1)}$. Quando 
$k$ tende a infinito (ou seja, quando $\mathbf{A}_{k}$ tende com probabilidade 1 à matrizidentidade), é possível verificar que $\mathbf{z} \equiv \mathbf{x}$, no sentido em que $\mathbf{z}=\mathbf{I}_{\mathbb{S}^{(i-1)}, \mathbb{S}^{(i)}} \mathbf{x}$. Seja $\mathbf{w}=\mathbf{I}_{\mathbb{S}^{(i-1)}, \mathbb{S}^{(i)}} \mathbf{y}$ (ou seja, $\left.\mathbf{w} \equiv \mathbf{y}\right)$. Portanto, observando que $\mathbb{V}^{(i)} \subset \mathbb{V}^{(i-1)} \mathrm{e}$ devido à propriedade triangular das normas, é possível afirmar que a distância $\left\|\mathbf{w}-\hat{\mathbf{h}}_{k}^{\mathbb{S}^{(i-1)}}\right\|_{1}$ é a máxima distância correspondente à variação de $\delta_{u}$ de 0 até $b$, dentro do sub-espaço $\mathbb{V}^{(i-1)}$. Além disso, é possível verificar que $J_{k}^{\mathbb{S}^{(i)}}(\mathbf{y})=J_{k}^{\mathbb{S}^{(i-1)}}(\mathbf{w})$.

Diante do exposto, conclui-se por indução que a solução $\tilde{\mathbf{h}}_{k}^{\mathbb{L}}$, quando $k$ tende a infinito, somente pode ser obtida através de encolhimentos sucessivos do suporte $\mathbb{L}$, conforme $4-65$, até que a condição $\delta_{u}<a^{(i)}$ seja atendida, com o valor de $\delta_{u}$ obtido através de 4-64. A Figura 4.27 ilustra os trajetos de máxima distância, considerando a transição entre os sub-espaços $\mathbb{V}^{(i-1)}$ e $\mathbb{V}^{(i)}$, com $\mathbb{V}^{(i)} \subset \mathbb{V}^{(i-1)}$.

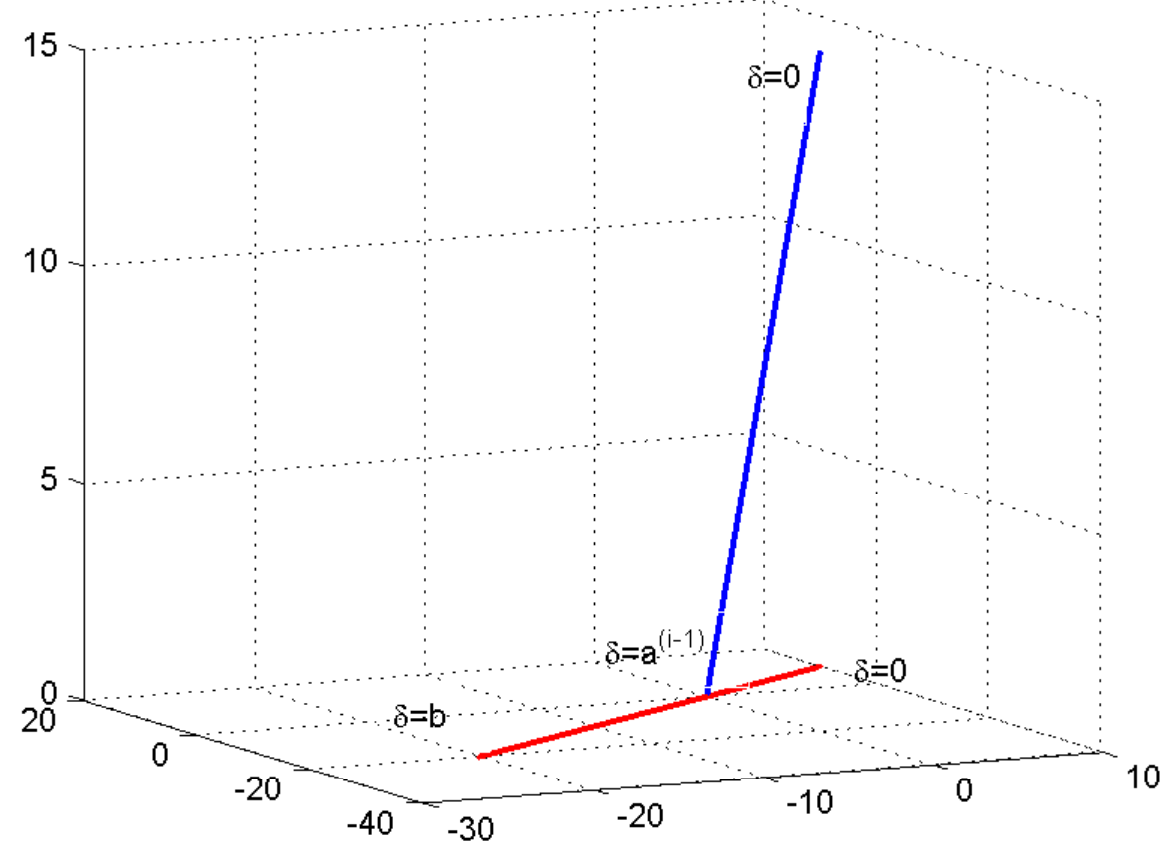

Figura 4.27: Transição entre os sub-espaços $\mathbb{V}^{(i-1)}$ e $\mathbb{V}^{(i)}$, com $\mathbb{V}^{(i)} \subset \mathbb{V}^{(i-1)}$. A curva azul representa o trajeto de máxima distância $l_{1}$, no sub-espaço $\mathbb{V}^{(i-1)}$, entre $\hat{\mathbf{h}}_{k}^{\mathbb{S}^{(i-1)}}\left(\delta_{u}=0\right)$ e o ponto de contato $\mathbf{z}$ com $\mathbb{V}^{(i)}\left(\delta_{u}=a^{(i-1)}\right)$. A curva vermelha representa o trajeto de máxima distância $l_{1}$, no sub-espaço $\mathbb{V}^{(i)}$, entre $\hat{\mathbf{h}}_{k}^{\mathbb{S}(i)}\left(\delta_{u}=0\right)$ e $\mathbf{y}\left(\delta_{u}=b\right)$, passando pelo ponto $\mathbf{x} \equiv \mathbf{z}\left(\delta_{u}=a^{(i-1)}\right)$. 


\section{Resumo do algoritmo}

O método proposto de Minimização da Norma $l_{1}$ pode ser descrito conforme o Algoritmo 4.7.

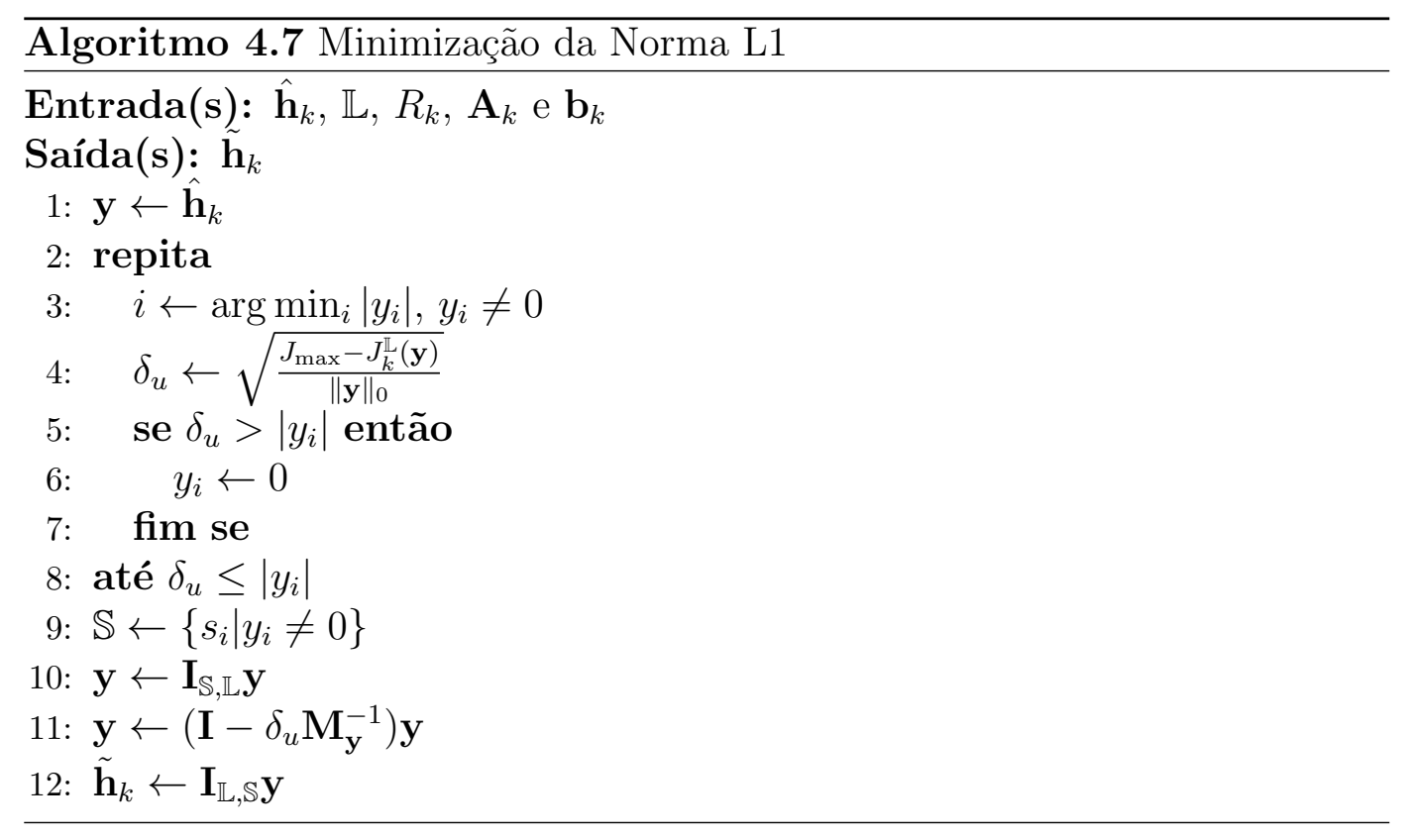

O algoritmo 4.7 gera estimativas polarizadas. Suas etapas 10 a 12 podem ser substituídas por uma etapa de despolarização, dando origem ao Algoritmo 4.8.

\subsection{3}

\section{Análise de convergência}

Quando $u$ tende a infinito, a solução $\tilde{\mathbf{h}}_{k}^{\mathbb{L}}$ ao problema enunciado em 4-40 converge para a solução ao problema original enunciado em 4-37. A expressão em 4-64 é monótona crescente. Quando $u=0, \delta_{u}=0$ (que corresponde ao ponto interior $\left.\hat{\mathbf{h}}_{k}^{\mathbb{L}}\right)$ e, quando $u$ tende a infinito, tem-se que

$$
\delta_{\infty}=\sqrt{\frac{J_{\max }-J_{k}(\mathbb{L})}{|\mathbb{L}|}}
$$

Comparando 4-66 com 4-62, verifica-se que, quando $u=\infty, J_{k}^{\mathbb{L}}\left(\tilde{\mathbf{h}}_{k}^{\mathbb{L}}\right)=J_{\max }$.

\subsection{4}

\section{Interpretação geométrica}

Geometricamente, o problema enunciado em 4-37 pode ser interpretado da seguinte forma: Seja $\mathbb{E}^{\mathbb{L}}\left(J_{\max }\right)=\left\{\mathbf{x} \mid J_{k}^{\mathbb{L}}(\mathbf{x}) \leq J_{\max }\right\}$ o lugar-geométrico 


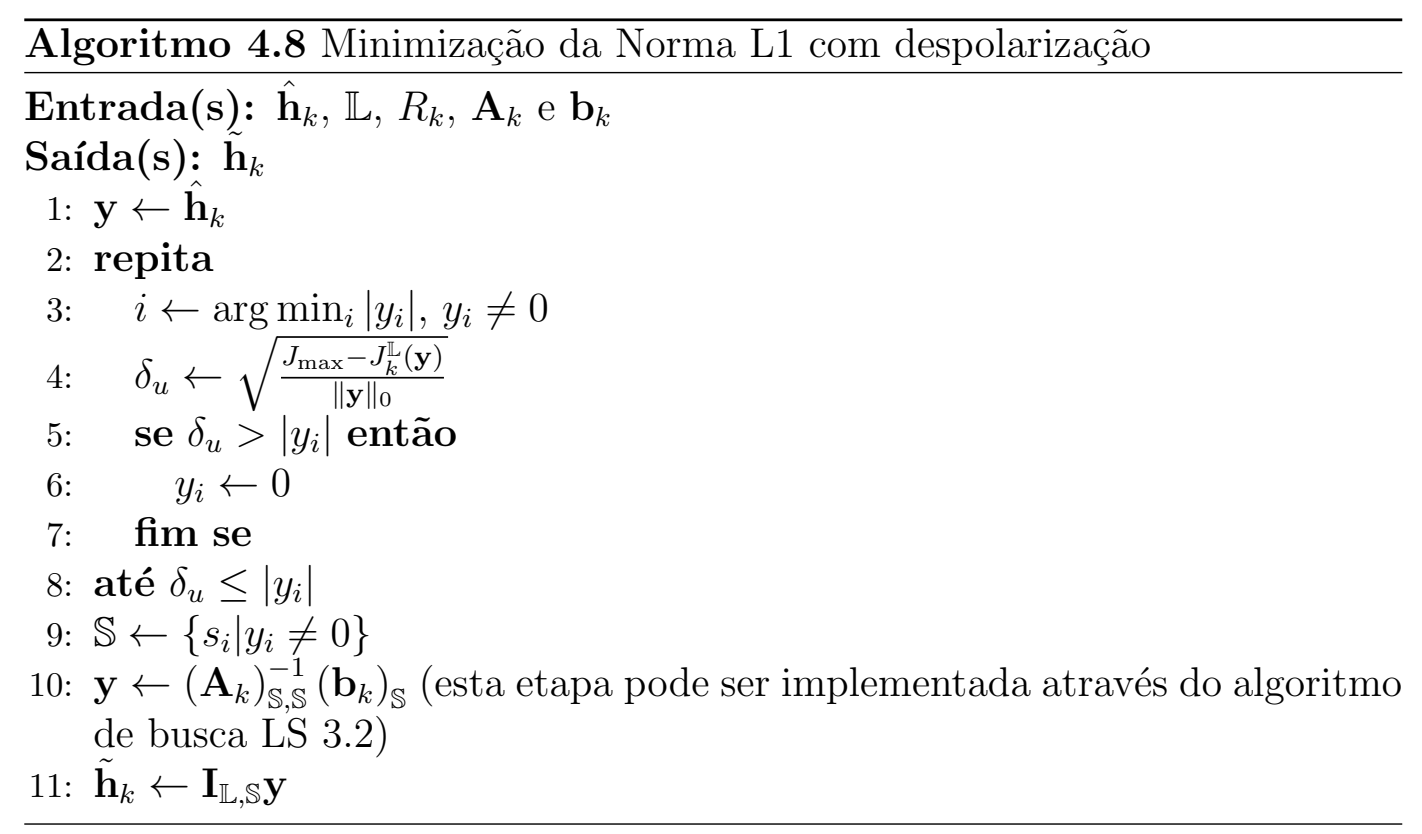

dos pontos no hiperespaço associado ao suporte $\mathbb{L}$ cujo custo LS é menor ou igual a $J_{\max }$. Nesse caso, $\mathbb{E}^{\mathbb{L}}\left(J_{\max }\right)$ é um hiper-elipsoide cujo centro é $\hat{\mathbf{h}}_{k}^{\mathbb{L}}$. Seja $\mathbb{P}^{\mathbb{L}}(c)=\left\{\mathbf{x} \mid\|\mathbf{x}\|_{1} \leq c\right\}$ o lugar-geométrico dos pontos no hiperespaço associado ao suporte $\mathbb{L}$ cuja norma $l_{1}$ é menor ou igual a uma constante $c$. Nesse caso, $\mathbb{P}^{\mathbb{L}}(c)$ corresponde a um hiper-poliedro cujo centro é a origem.

Caso $J_{k}^{\mathbb{L}}(\mathbf{0})=R_{k} \leq J_{\max }$, então o hiper-elipsoide contém a origem e, portanto, a solução trivial de 4-37 é $\tilde{\mathbf{h}}_{k}^{\mathbb{L}}=\mathbf{0}$; caso contrário, $\tilde{\mathbf{h}}_{k}^{\mathbb{L}}$ corresponde ao único ponto de contato entre a superfície do hiper-elipsoide $\mathbb{E}^{\mathbb{L}}\left(J_{\max }\right)$ e a superfície do único hiper-poliedro $\mathbb{P}^{\mathbb{L}}(c)$ que tangencia $\mathbb{E}^{\mathbb{L}}\left(J_{\max }\right)$. Portanto nesse caso, $\left\|\tilde{\mathbf{h}}_{k}^{\mathbb{L}}\right\|_{1}=c$ e $J_{k}^{\mathbb{L}}\left(\tilde{\mathbf{h}}_{k}^{\mathbb{L}}\right)=J_{\max }$. A unicidade de $\tilde{\mathbf{h}}_{k}^{\mathbb{L}}$ é garantida pelo fato de $\mathbb{P}^{\mathbb{L}}(c)$ ser convexa para todo $c \geq 0$ e $\mathbb{E}^{\mathbb{L}}\left(J_{\max }\right)$ ser estritamente convexa. A Figura 4.28 ilustra esta condição de contorno.

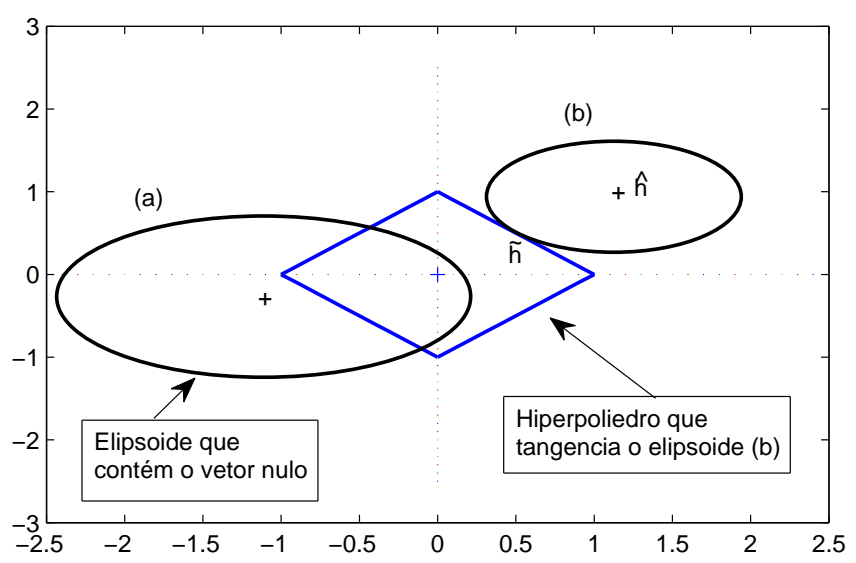

Figura 4.28: Unicidade da estimativa referente à minimização da norma $l_{1}$, restrita ao custo LS menor que $J_{\max }$ : (a) $J_{k}^{\mathbb{L}}(\mathbf{0}) \leq J_{\max }$, (b) $J_{k}^{\mathbb{L}}(\mathbf{0})>J_{\max }$ 
O ponto de tangência $\tilde{\mathbf{h}}_{k}^{\mathbb{L}}$ entre o hiperelipsóide $\mathbb{E}^{\mathbb{L}}\left(J_{\max }\right)$ e o hiperpoliedro $\mathbb{P}^{\mathbb{L}}(c)$ pode estar localizado ou em uma das faces ou em uma das arestas ou em um dos vértices de $\mathbb{P}^{\mathbb{L}}(c)$. Quando $\tilde{\mathbf{h}}_{k}^{\mathbb{L}}$ é um dos vértices de $\mathbb{P}^{\mathbb{L}}(c)$, então apenas um dos seus elementos é diferente de zero; quando $\tilde{\mathbf{h}}_{k}^{\mathbb{L}}$ está localizado em uma das arestas de $\mathbb{P}^{\mathbb{L}}(c)$, então $\tilde{\mathbf{h}}_{k}^{\mathbb{L}}$ contém um ou mais elementos nulos; $\tilde{\mathbf{h}}_{k}^{\mathbb{L}}$ esta localizado no interior de uma das faces de $\mathbb{P}^{\mathbb{L}}(c)$, então todos os seus elementos são diferentes de zero. A Figura 4.29 ilustra as três possibilidades de tangência entre o hiperelipsóide $\mathbb{E}^{\mathbb{L}}\left(J_{\max }\right)$ e o hiperpoliedro $\mathbb{P}^{\mathbb{L}}(c)$.

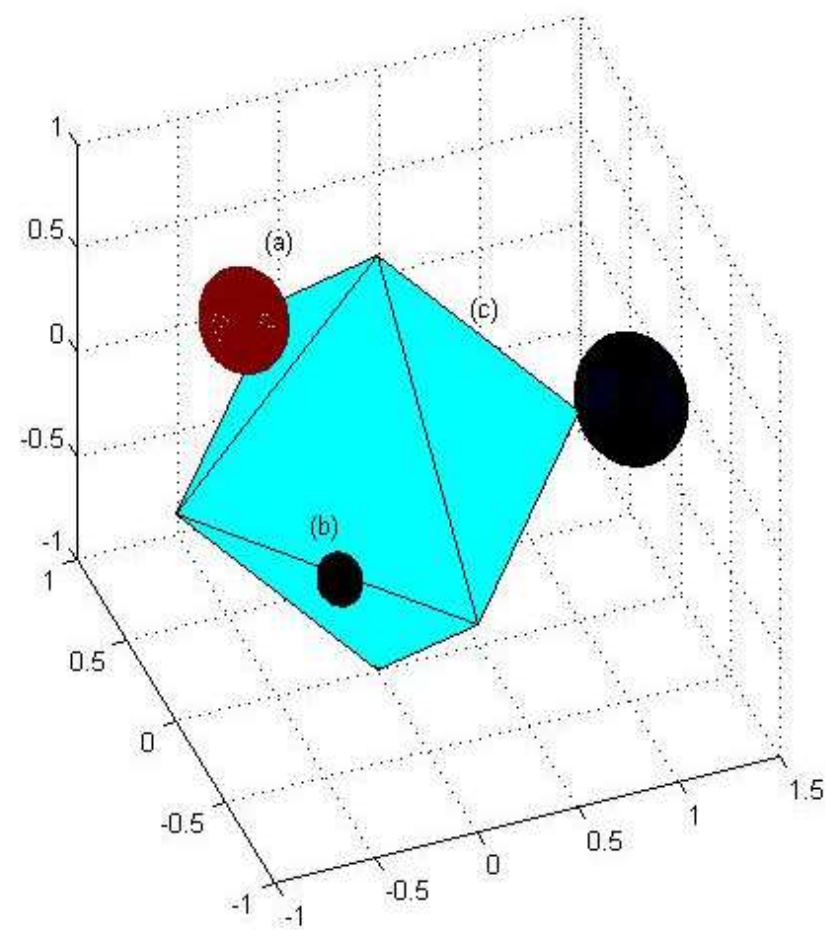

Figura 4.29: Tangência do hiper-elipsoide com: (a) face do hiperpoliedro; (b) aresta do hiperpoliedro; (c) vértice do hiperpoliedro

Quando $k$ tende a infinito, os hiperelipsóides $\mathbb{E}^{\mathbb{L}} \tilde{\mathbf{h}}\left(J_{\max }\right)$ e $\mathbb{E}^{\mathbb{L}}\left(J_{\max }\right)$ degeneramse em hiperesferas, tendo como pontos centrais $\hat{\mathbf{h}}_{k}^{\mathbb{L}} \tilde{\tilde{h}}$ e $\hat{\mathbf{h}}_{k}^{\mathbb{L}}$, respectivamente, sendo que $\hat{\mathbf{h}}_{k}^{\mathbb{L}_{\tilde{\mathbf{h}}}}$ corresponde à projeção de $\hat{\mathbf{h}}_{k}^{\mathbb{L}}$ no sub-espaço associado a $\mathbb{L}_{\tilde{\mathbf{h}}}$.

A seguinte analogia pode ser feita em relação ao Algoritmo 4.8, para determinação de $\tilde{\mathbf{h}}_{k}^{\mathbb{L}}$ : seja uma represa totalmente cheia, onde na $i$-ésima posição $(i=1,2, \ldots,|\mathbb{L}|)$ a profundidade é igual a $\left|\left(\hat{\mathbf{h}}_{k}^{\mathbb{L}}\right)_{i}\right|$, determinada pelo terreno 
irregular do seu fundo. Nesse caso, a superfície da represa cheia corresponde a $\delta_{u}=0$. À medida em que o nível da represa baixa (ou seja, à medida em que $\delta_{u}$ aumenta), alguns pontos do fundo da represa passam a ficar descobertos (a profundidade nesses pontos da represa passa a ser zero). Além disso, à medida em que $\delta_{u}$ aumenta (ou seja, o nível de água da represa baixa), $J_{k}^{\mathbb{L}}\left(\tilde{\mathbf{h}}_{k}^{\mathbb{L}}\right)$ aumenta, haja vista que, quanto maior $\delta_{u}$, maior $\left\|\hat{\mathbf{h}}_{k}^{\mathbb{L}}-\tilde{\mathbf{h}}_{k}^{\mathbb{L}}\right\|^{2}$. A Figura 4.30 ilustra essa analogia.
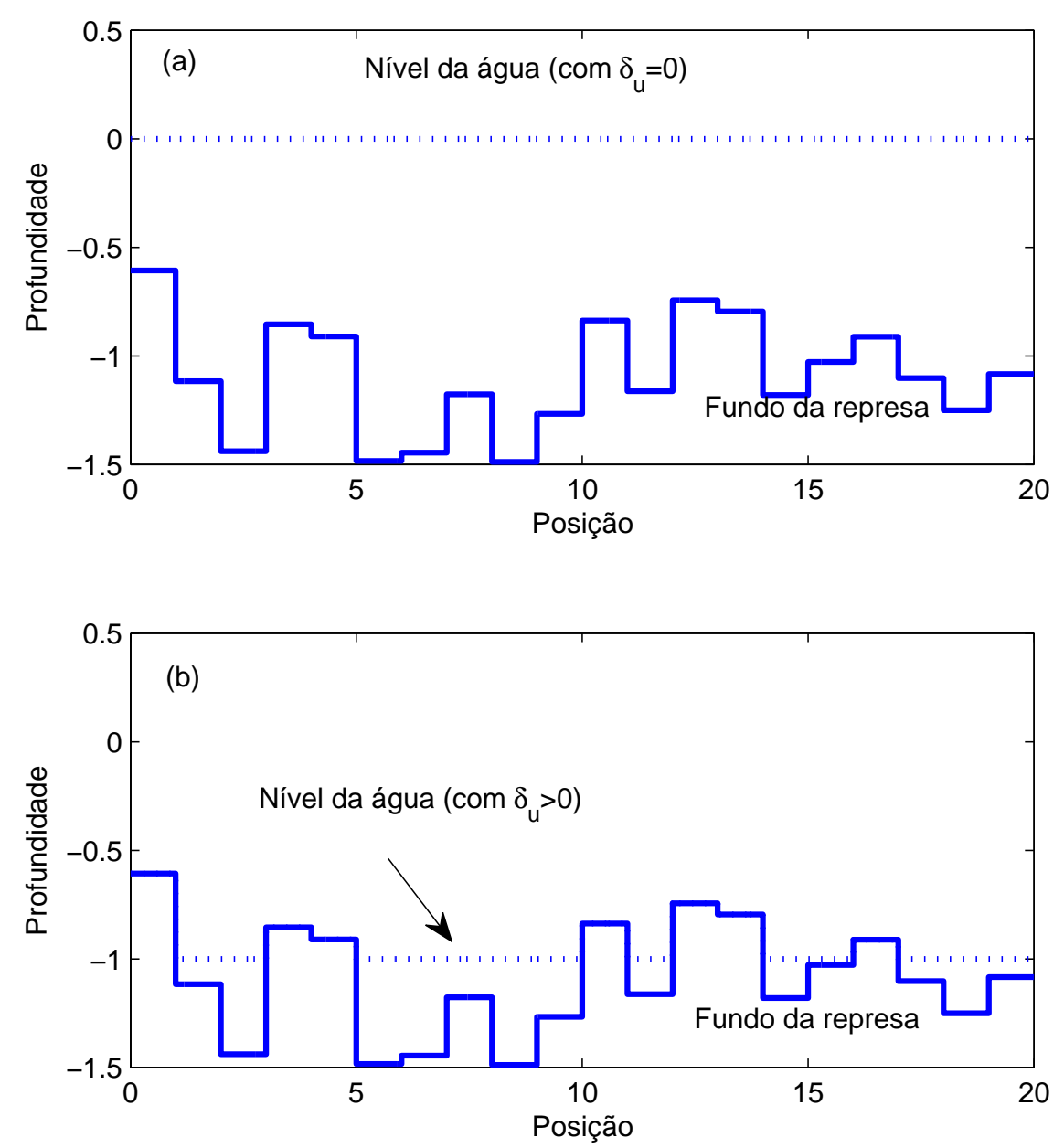

Figura 4.30: Analogia do Método de Minimização da Norma $l_{1}$ com a redução do nível de água em uma represa. Neste exemplo $|\mathbb{L}|=20$, onde: (a) se $\delta_{u}=0$, então $J_{k}^{\mathbb{L}}\left(\tilde{\mathbf{h}}_{k}\right)=J_{k}(\mathbb{L})$; (b) se $\delta_{u}>0$, então $J_{k}^{\mathbb{L}}\left(\tilde{\mathbf{h}}_{k}\right)>J_{k}(\mathbb{L})$. À medida em que $\delta_{u}$ aumenta, $J_{k}^{\mathbb{L}}\left(\tilde{\mathbf{h}}_{k}\right)$ também aumenta, ao mesmo tempo em que os elementos de $\tilde{\mathbf{h}}_{k}$ são encolhidos. Dependendo do valor de $\delta_{u}$, alguns elementos de $\tilde{\mathbf{h}}_{k}$ podem ser zerados, o que é ilustrado em (b) pelas superfícies do fundo da represa acima do nível de água. 
Cabe aqui destacar que o método proposto de Minimização da Norma $l_{1}$ é, surpreendentemente, muito semelhante ao método de Encolhimentos Sucessivos, haja vista que ambos constroem suas estimativas a partir da decimação dos elementos de $\hat{\mathbf{h}}_{k}^{\mathbb{L}}$, começando pelo elemento com o menor valor absoluto e prosseguindo em direção ao elemento com o maior valor absoluto. Entretanto, enquanto que no método de encolhimentos sucessivos o critério de decimação se justifica pelo fato de que a variância do desvio de ruído tende a zero à medida em que $k$ tende a infinito, no método de minimização da norma $l_{1}$ o critério de decimação se justifica pelas condições geométricas de contorno que o ponto de tangência $\tilde{\mathbf{h}}_{k}^{\mathbb{L}}$ entre $\mathbb{E}^{\mathbb{L}}\left(J_{\max }\right)$ e $\mathbb{P}^{\mathbb{L}}(c)$ deve atender. Apesar das diferentes argumentações, estes dois métodos apresentam uma forte conexão entre si.

\subsection{5}

\section{Custo computacional}

Os passos 2 a 8 dos algoritmos 4.7 e 4.8 são muito semelhantes ao algoritmo de encolhimentos sucessivos. Os passos 9 a 12 do algoritmo 4.7 substituem a etapa de despolarização encontrada no método de encolhimentos sucessivos e têm custo computacional $\mathcal{O}(|\mathbb{S}|$ ) (bem menor que o custo para o cálculo da estimativa LS com suporte reduzido, que é $\mathcal{O}\left(|\mathbb{S}|^{3}\right)$ ), haja vista que $\mathbf{M}_{\mathbf{y}}$ é matriz-diagonal. Por outro lado, o algoritmo 4.8 também possui uma etapa de despolarização e, por isso, seu custo computacional é um pouco maior que do algoritmo de encolhimentos sucessivos.

\subsection{6}

\section{Avaliação de desempenho}

O algoritmo proposto, em sua versão sem despolarização, foi projetado admitindo-se $\mathbf{A}_{k}=\mathbf{I}$ (o que ocorre quando $k=\infty$ ) e $u=\infty$ (que é a condição para que a solução $\tilde{\mathbf{h}}_{k}^{\mathbb{S}}$ ao problema enunciado em 4-40 coincida com a solução ao problema original enunciado em 4-37), quando $J_{k}^{\mathbb{S}}\left(\tilde{\mathbf{h}}_{k}^{\mathbb{S}}\right)=J_{\max }$.

A Figura 4.31 mostra o desvio normalizado médio do custo LS em relação a $J_{\max }$ em função do tamanho da sequência de treinamento $K$, estimado sobre $Q=1000$ realizações independentes de um sistema $\operatorname{MESS}(100, \mathrm{~S}), \operatorname{com} S=10$ e $S=20, N=32, \lambda=1,01$ e SINR igual a $6 \mathrm{~dB}$ e $20 \mathrm{~dB}$. O desvio normalizado médio do custo LS em relação a $J_{\max }$ é definido como

$$
N M S D_{J}(k)=E\left[\left|\frac{J_{k}^{\mathbb{L}}\left(\tilde{\mathbf{h}}_{k}\right)-J_{\max }}{J_{\max }}\right|^{2}\right]
$$


A Figura 4.31 mostra que, nas simulações executadas, independente do valor de SINR, o desvio normalizado médio do custo LS permaneceu pequeno mesmo para valores de $K$ relativamente pequenos.

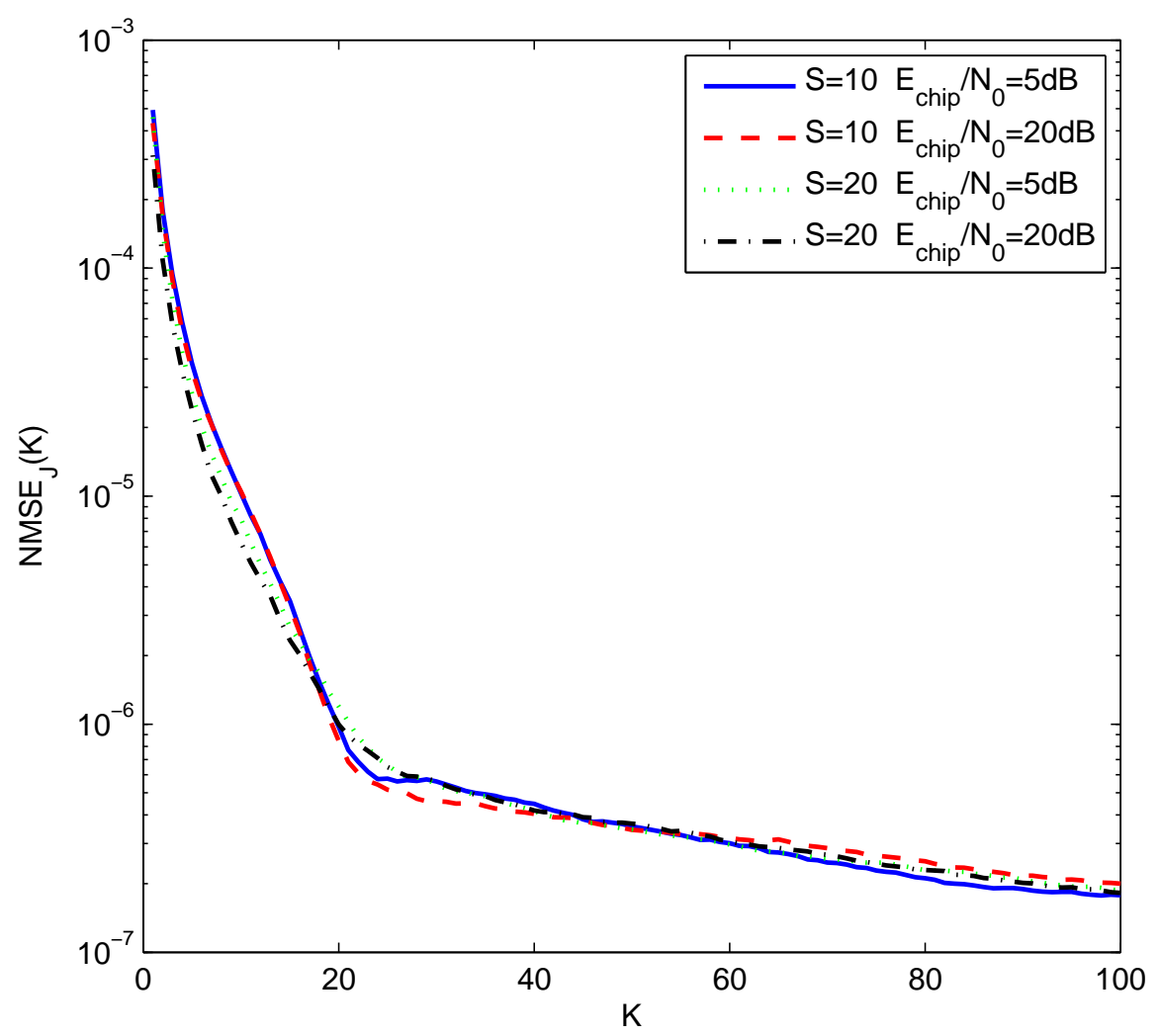

Figura 4.31: NMSD do custo LS para sistema $\operatorname{MESS}(100, S)$, com $S=10$ e $S=20, N=32, \lambda=1,01$ e $Q=1000$

As Figuras 4.32, 4.33, 4.34 e 4.35 mostram o desempenho médio do algoritmo proposto, nas versões sem e com despolarização, comparado com o desempenho das estimações LS Full-support e LS oráculo, em função do comprimento $K$ da sequência de treinamento e avaliado sobre $Q=1000$ realizações independentes de sistema $\operatorname{MESS}(100,10), \operatorname{com} N=16, \lambda=1,05$ e $\operatorname{SINR}=15,20,25$ e 30 dB respectivamente. Nos gráficos superiores, são mostrados os desempenhos dos algoritmos citados em termos de NMSD. Nos gráficos inferiores, são mostradas as probabilidades de ocorrência dos seguintes tipos de encolhimento: correto, pequeno, excessivo e disforme. As Figuras 4.36, 4.37, 4.38 e 4.39 mostram o desempenho médio do algoritmo proposto, nas versões sem e com despolarização, para MESS(100,20) e mantendo-se os parâmetros de simulação restantes inalterados. 

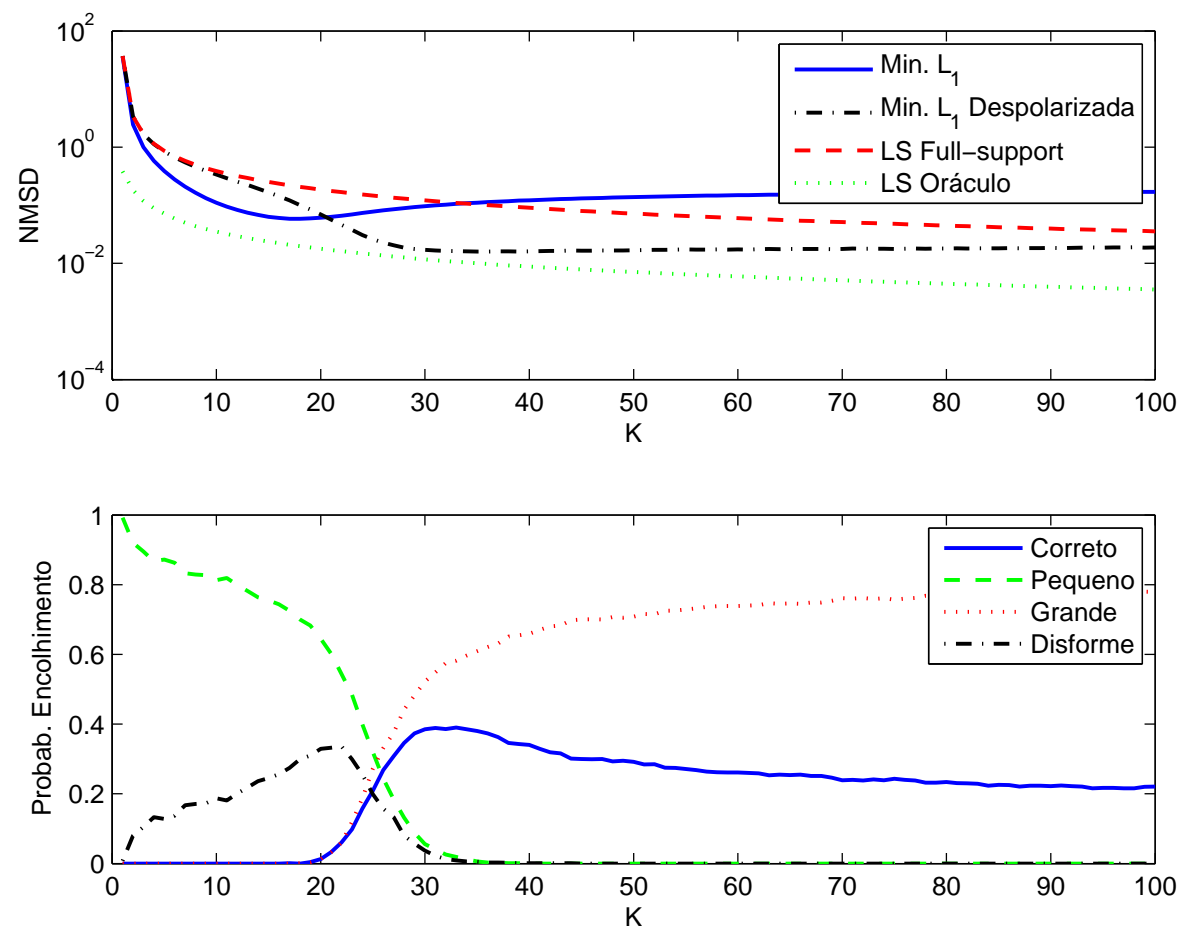

Figura 4.32: Min. $l_{1}$ : $\operatorname{MESS}(100,10), \operatorname{SINR}=15 \mathrm{~dB}, \lambda=1,05, \mathrm{~N}=16$ e $\mathrm{Q}=1000$
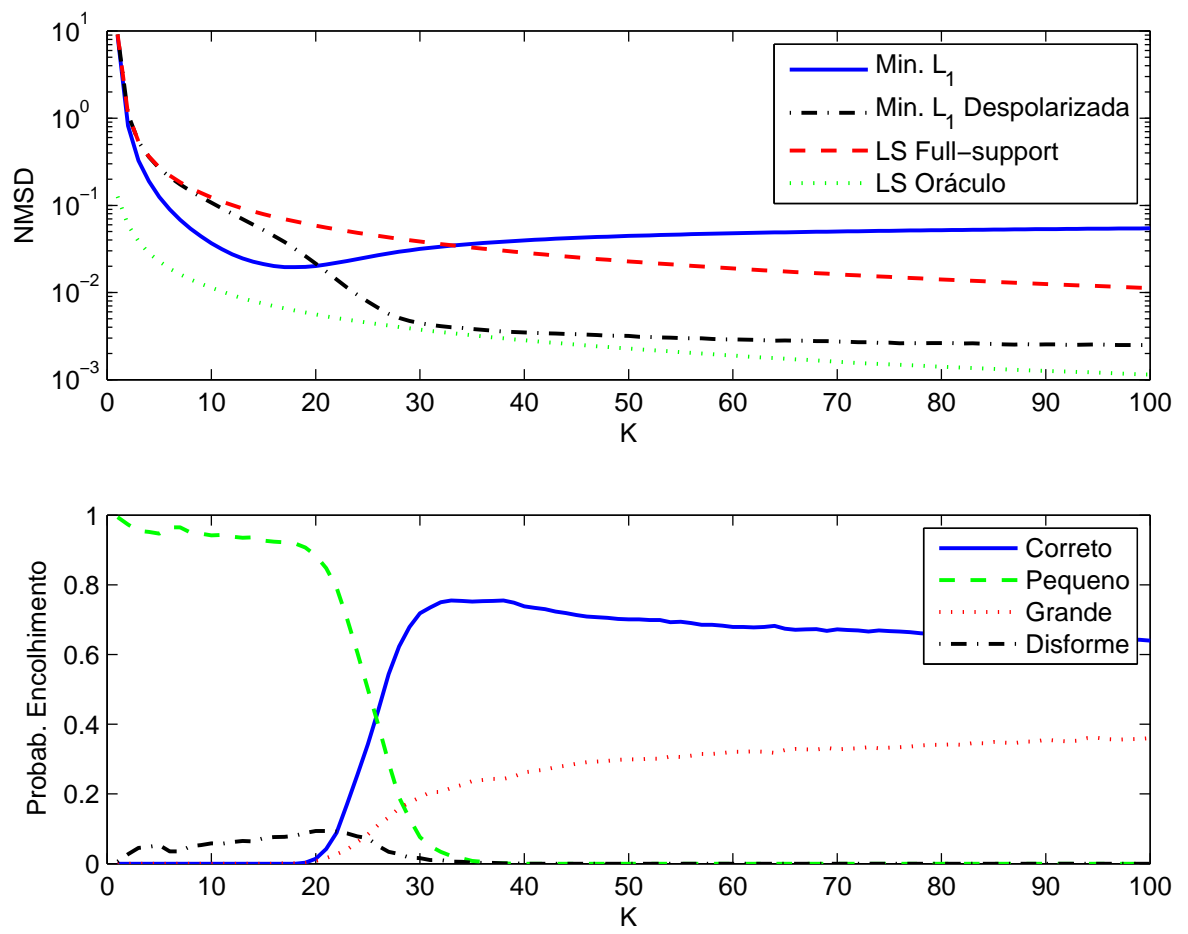

Figura 4.33: Min. $l_{1}$ : $\operatorname{MESS}(100,10), \operatorname{SINR}=20 \mathrm{~dB}, \lambda=1,05, N=16$ e $\mathrm{Q}=1000$ 

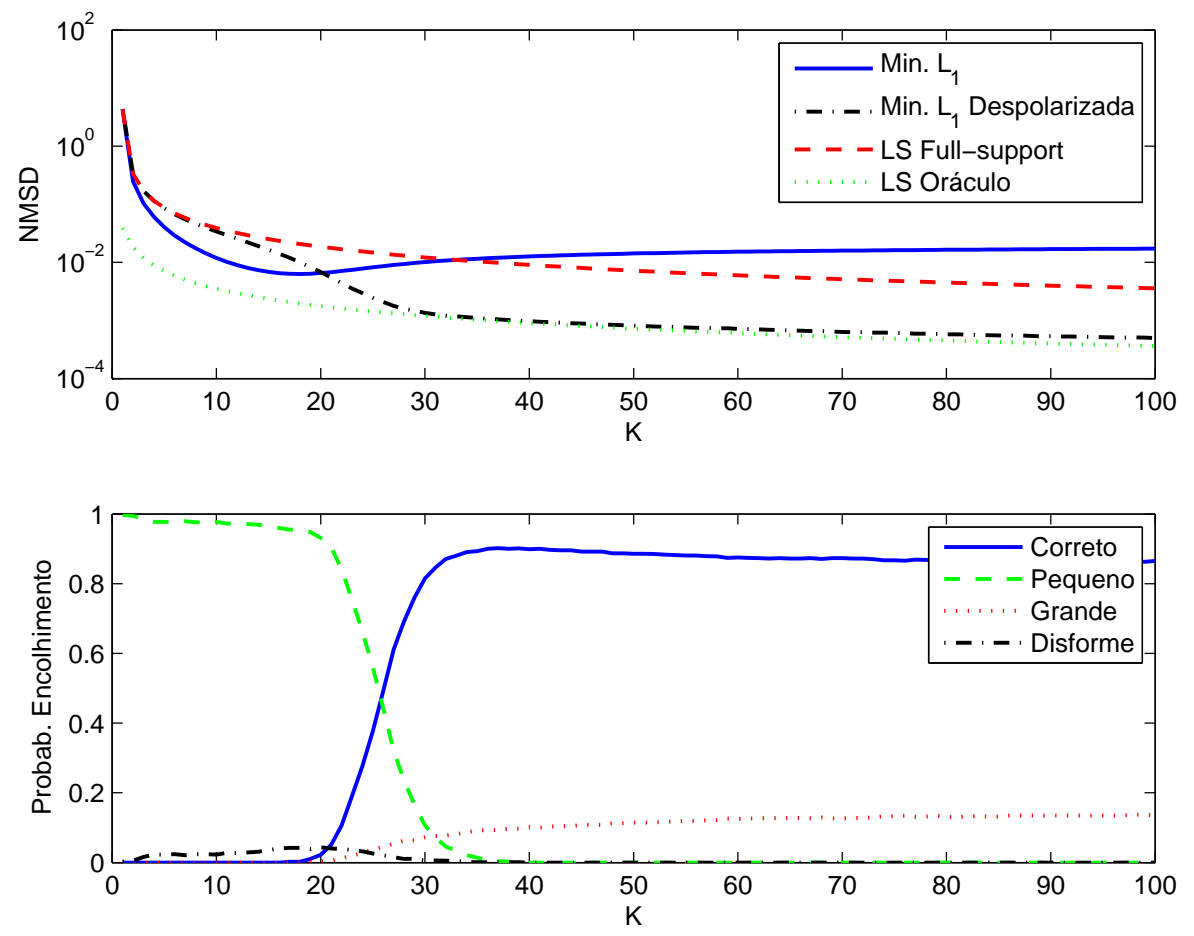

Figura 4.34: Min. $l_{1}$ : $\operatorname{MESS}(100,10), \mathrm{SINR}=25 \mathrm{~dB}, \lambda=1,05, \mathrm{~N}=16$ e $\mathrm{Q}=1000$
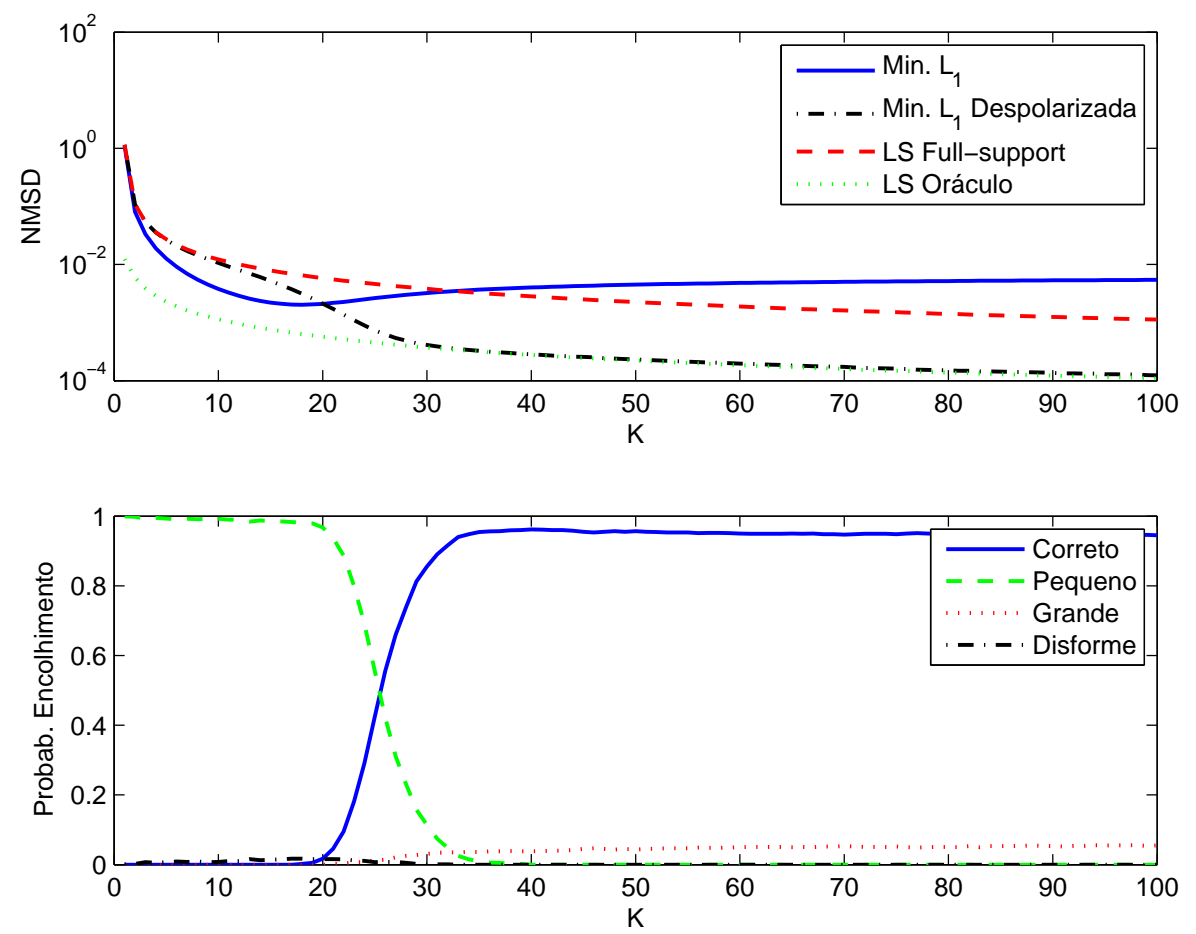

Figura 4.35: Min. $l_{1}$ : $\operatorname{MESS}(100,10), \operatorname{SINR}=30 \mathrm{~dB}, \lambda=1,05, N=16$ e $\mathrm{Q}=1000$ 

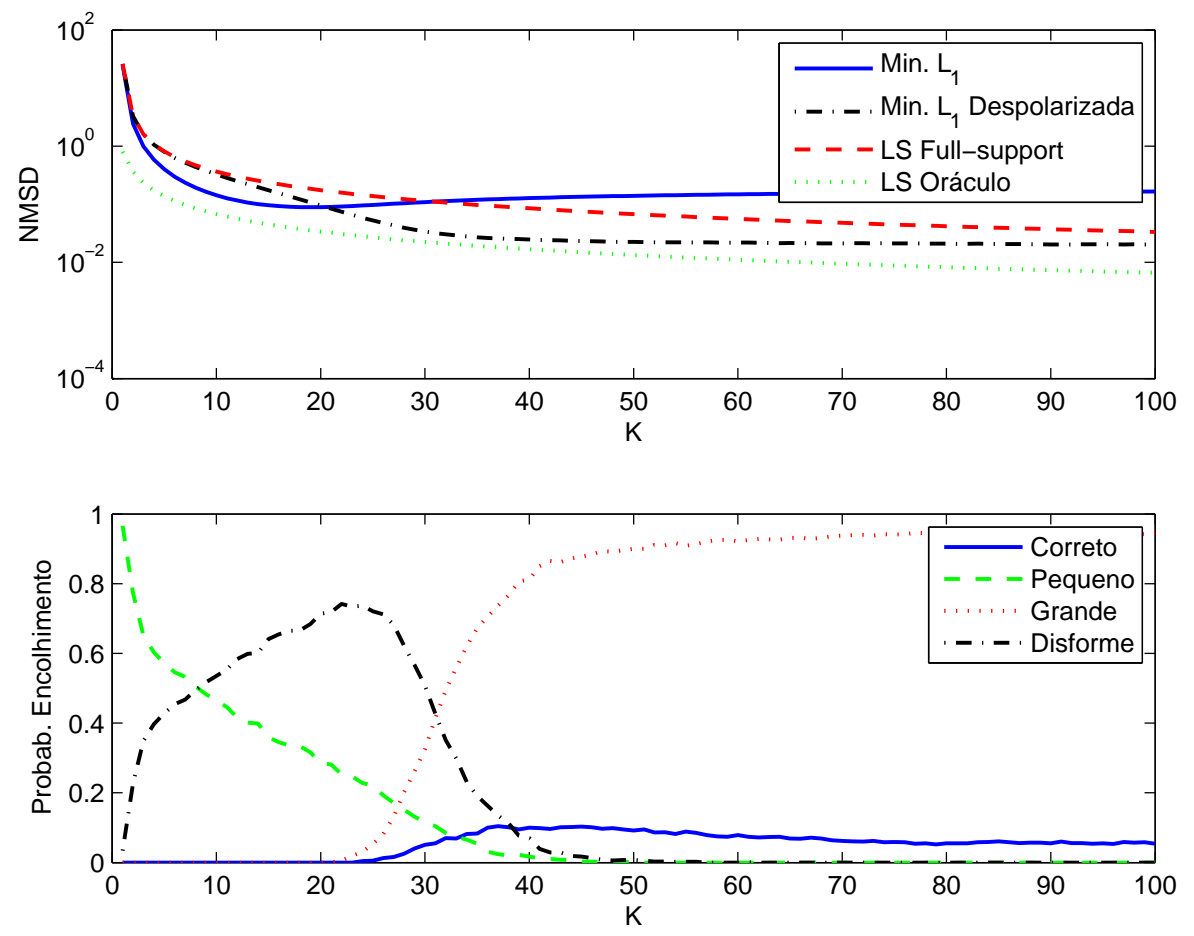

Figura 4.36: Min. $l_{1}$ : $\operatorname{MESS}(100,20), \operatorname{SINR}=15 \mathrm{~dB}, \lambda=1,05, \mathrm{~N}=16$ e $\mathrm{Q}=1000$
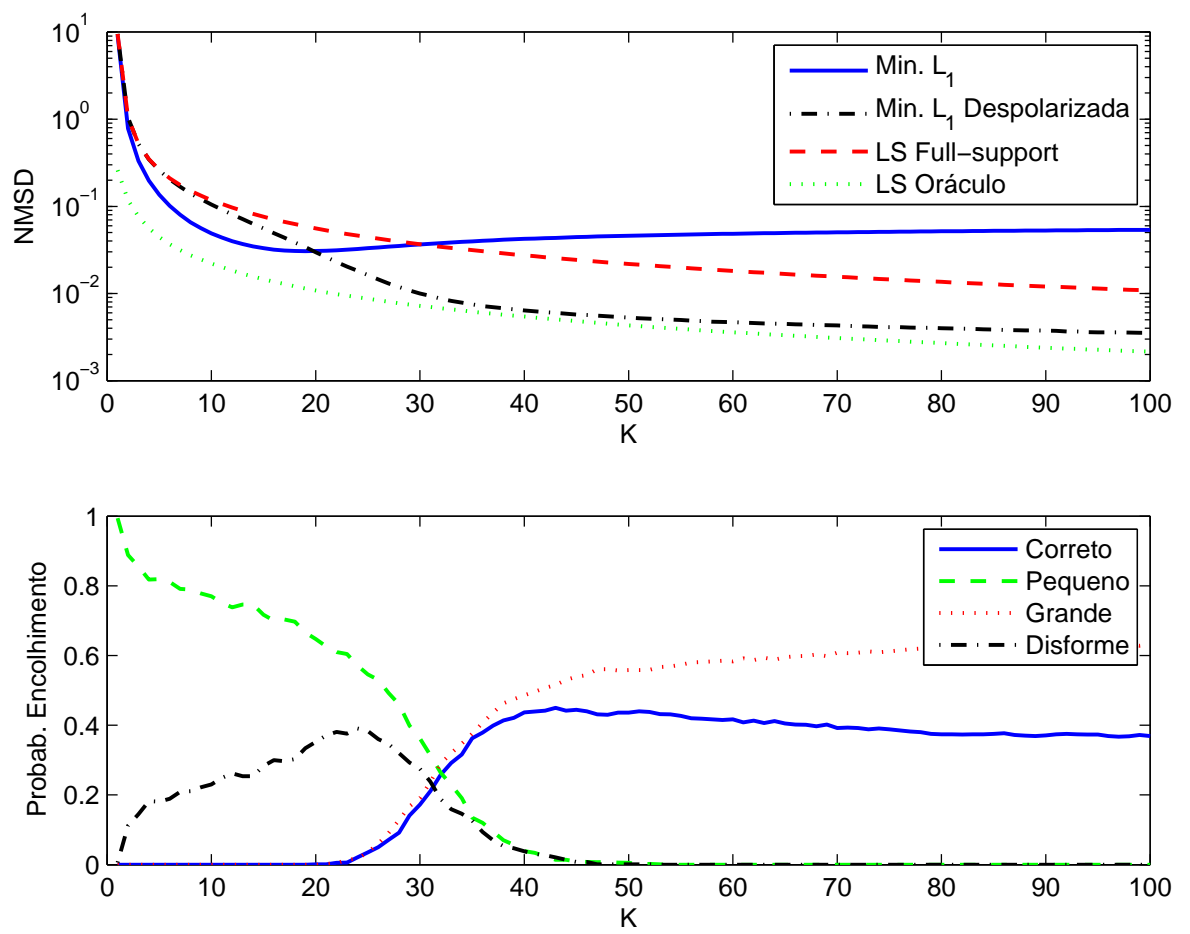

Figura 4.37: Min. $l_{1}$ : $\operatorname{MESS}(100,20), \operatorname{SINR}=20 \mathrm{~dB}, \lambda=1,05, N=16$ e $\mathrm{Q}=1000$ 

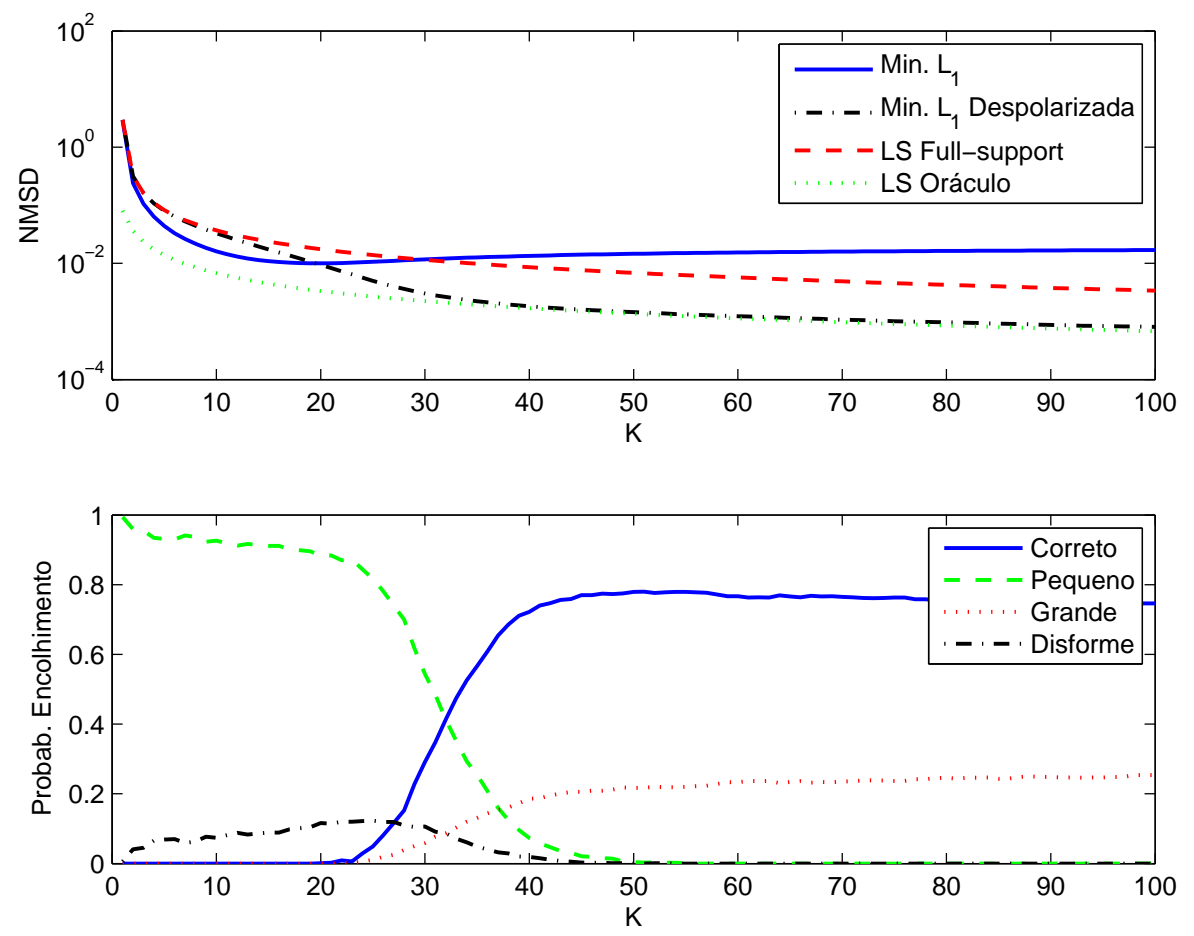

Figura 4.38: Min. $l_{1}$ : $\operatorname{MESS}(100,20), \mathrm{SINR}=25 \mathrm{~dB}, \lambda=1,05, \mathrm{~N}=16$ e $\mathrm{Q}=1000$
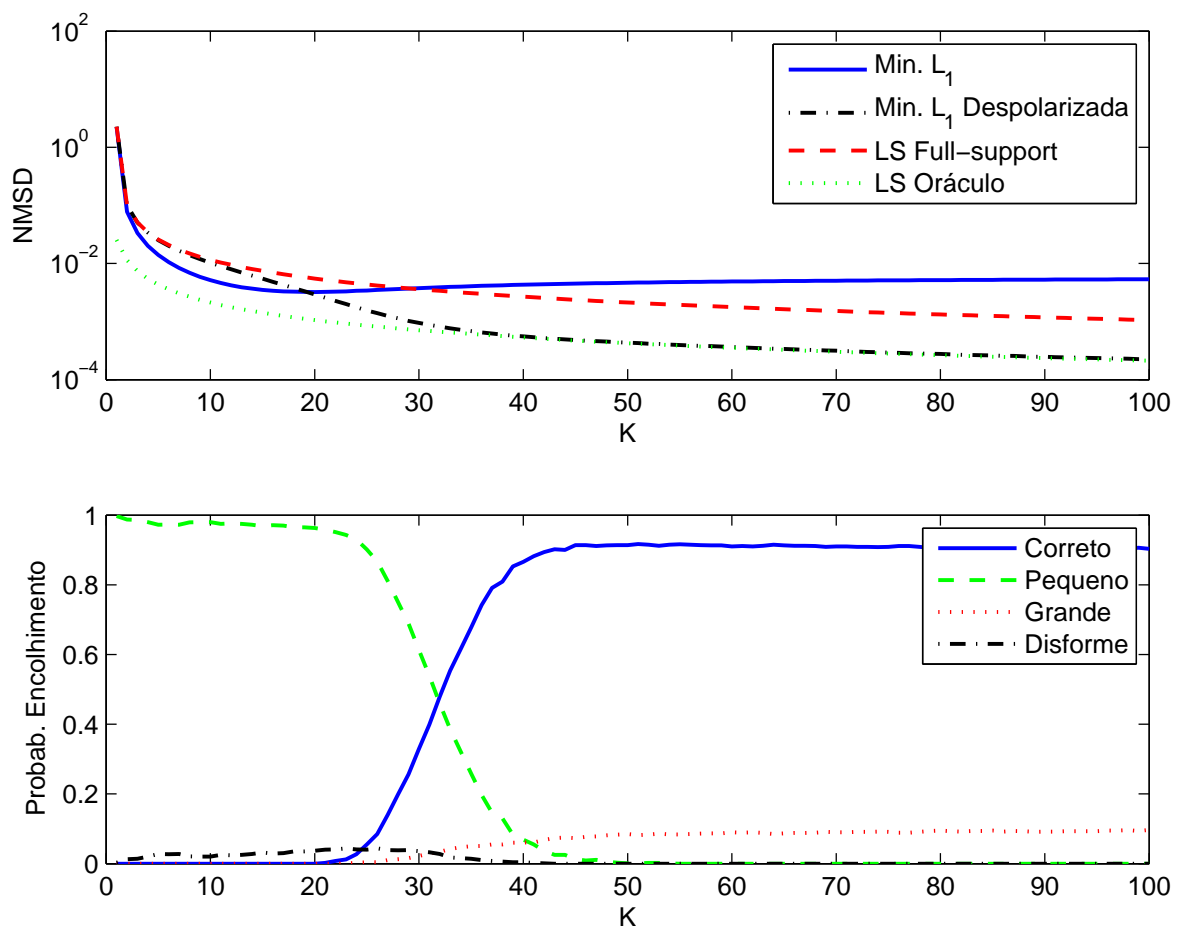

Figura 4.39: Min. $l_{1}$ : $\operatorname{MESS}(100,20), \operatorname{SINR}=30 \mathrm{~dB}, \lambda=1,05, N=16$ e $\mathrm{Q}=1000$ 
As figuras mostram que, quanto maior SINR ou mais esparso o sistema estimado, menores as probabilidades de ocorrências de sub-encolhimentos e encolhimentos disformes, aumentando-se assim o desempenho do algoritmo proposto.

\section{8}

\section{Método de Regularização Automática do custo LS}

\subsection{1}

\section{Introdução}

A técnica de regularização do custo LS consiste em adicionar uma função de penalidade à função-custo $J_{k}^{\mathbb{L}}(\mathbf{x})$, formando uma nova função-custo $\tilde{J}_{k}^{\gamma}(\mathbf{x})$ : $\mathbb{C}^{|\mathbb{L}|} \rightarrow \mathbb{R}$, dada por

$$
\tilde{J}_{k}^{\gamma}(\mathbf{x})=J_{k}^{\mathbb{L}}(\mathbf{x})+\gamma f(\mathbf{x})
$$

onde $f(\mathbf{x}): \mathbb{C}^{|\mathbb{L}|} \rightarrow \mathbb{R}$ é uma função de penalidade (também chamada de função de regularização) e $\gamma \in \mathbb{R}^{+}$é o fator de penalidade (ou fator de regularização) $(17,20)$.

A função de penalidade $f(\mathbf{x})$ é projetada para que, quanto maior o grau de esparsidade de $\mathbf{x}$, menor seu valor. O grau de esparsidade pode ser medido através de funções baseadas na pseudo-norma $l_{0}$ ou na norma $l_{1}$, no sentido de que, quanto menor $\|\mathbf{x}\|_{d}$, com $d \in\{0,1\}$, maior o grau de esparsidade de $\mathbf{x}$.

Para todo $\gamma \geq 0$, a estimativa da resposta ao impulso discreta do sistema é dada por

$$
\tilde{\mathbf{h}}_{k}(\gamma)=\arg \min _{\mathbf{x}} \tilde{J}_{k}^{\gamma}(\mathbf{x})
$$

\subsection{2}

\section{O valor ótimo do fator de regularização}

Nos métodos de regularização mais comumente encontrados na literatura, o valor de $\gamma$ é fixado em função da função de penalidade adotada e do modelo estocástico da resposta ao impulso do sistema estimado. Entretanto, essa abordagem possui duas características indesejadas: a escolha do valor de $\gamma$ é usualmente empírica e; como o valor de $\gamma$ é fixo, não é otimizado para cada realização individual da resposta ao impulso do sistema estimado.

Neste trabalho, o valor ótimo de $\gamma$, aqui denominado de $\gamma^{*}$, é definido como 


$$
\gamma^{*} \triangleq \arg _{\gamma} J_{k}^{\mathbb{L}}\left(\tilde{\mathbf{h}}_{k}(\gamma)\right)=J_{\max }
$$

onde $\tilde{\mathbf{h}}_{k}(\gamma)$ é a solução de 4-69 e $J_{\max }$ é o custo LS admitido, com $J_{k}(\mathbb{L})<$ $J_{\max }<J_{k}(\mathbf{0})$. O valor de $J_{\max }$ é escolhido conforme critério estabelecido na seção 4.2.1. Como será mostrado na seção 4.8.4, $J_{k}\left(\tilde{\mathbf{h}}_{k}(\gamma)\right)$ cresce monotonamente em função de $\gamma$ e, portanto, existe um único valor de $\gamma^{*}$ que satisfaz $4-70$.

Seja $\mathbf{h}_{k}^{*}$ o valor ótimo da estimativa esparsa (associado a $\gamma^{*}$ ), dado por

$$
\mathbf{h}_{k}^{*}=\tilde{\mathbf{h}}_{k}\left(\gamma^{*}\right)=\arg \min _{\mathbf{x}} \tilde{J}_{k}^{\gamma^{*}}(\mathbf{x})
$$

Como $\tilde{J}_{k}^{\gamma}($.$) é função convexa para todo \gamma$, então a solução de 4-71 é única. O fato de $\gamma$ pertencer ao intervalo ilimitado $[0, \infty)$ pode dificultar a obtenção de $\gamma^{*}$, o que motiva o emprego de homotopia entre o custo LS e a função de regularização.

\subsection{3}

\section{Regularização do custo LS por homotopia}

Seja a função convexa $\check{J}_{k}^{\rho}(\mathbf{x}): \mathbb{C}^{|\mathbb{L}|} \rightarrow \mathbb{R}$, com $\rho \in[0,1]$, dada por

$$
\check{J}_{k}^{\rho}(\mathbf{x})=\check{J}_{k}(\mathbf{x}, \rho) \triangleq(1-\rho) J_{k}^{\mathbb{L}}(\mathbf{x})+\rho f(\mathbf{x})
$$

A função $\breve{J}_{k}^{\rho}(\mathbf{x})$ estabelece uma homotopia entre $J_{k}^{\mathbb{L}}(\mathbf{x})$ e $f(\mathbf{x})$, ou seja, variando-se $\rho$ continuamente de zero até um, $\breve{J}_{k}^{\rho}(\mathbf{x})$ transforma-se continuamente de $J_{k}^{\mathbb{L}}(\mathbf{x})$ para $f(\mathbf{x})$.

Como $0 \leq \rho \leq 1, \breve{J}_{k}^{\rho}($.$) constitui-se em uma combinação afim de duas funções$ convexas e, portanto, também é convexa. Logo, $\breve{J}_{k}^{\rho}($.$) possui um único mínimo$ global, dado por

$$
\check{\mathbf{h}}_{k}(\rho)=\arg \min _{\mathbf{x}} \check{J}_{k}(\mathbf{x}, \rho)
$$

Comparando 4-68 com 4-72, é possível estabelecer a seguinte relação de equivalência

$$
\tilde{\mathbf{h}}_{k}(\gamma)=\check{\mathbf{h}}_{k}(\rho) \Leftrightarrow \gamma=\frac{\rho}{1-\rho}
$$

É importante observar em 4-74 que, para cada valor de $\gamma$ no intervalo ilimitado $[0, \infty)$, existe um único valor correspondente de $\rho$ no intervalo limitado $[0,1]$, e vice-versa. 


\subsection{4}

\section{Variação do custo LS e da penalidade em função do fator de regularização}

Sejam as funções $g(\rho), p(\rho)$ e $q(\rho)$ definidas a seguir para $\rho \in[0,1]$

$$
\begin{aligned}
& g(\rho) \triangleq \check{J}_{k}^{\rho}\left(\check{\mathbf{h}}_{k}(\rho)\right) \\
& p(\rho) \triangleq J_{k}^{\mathbb{L}}\left(\check{\mathbf{h}}_{k}(\rho)\right) \\
& q(\rho) \triangleq f\left(\check{\mathbf{h}}_{k}(\rho)\right)
\end{aligned}
$$

Nesse caso, é possível demonstrar o seguinte teorema:

Teorema 4.4 A função $g(\rho)$ é côncava.

Prova. Por definição, $g($.$) é função côncava se e somente se, para todo a e todo$ $b$ pertencentes ao dominio de $g($.$) e \theta \in[0,1], \theta g(a)+(1-\theta) g(b) \leq g(c)$, com $c=$ $\theta a+(1-\theta) b$. Segue que $\theta g(a)+(1-\theta) g(b)=\theta \check{J}_{k}^{a}\left(\check{\mathbf{h}}_{k}(a)\right)+(1-\theta) \check{J}_{k}^{b}\left(\check{\mathbf{h}}_{k}(b)\right) \leq$ $\theta \check{J}_{k}^{a}\left(\check{\mathbf{h}}_{k}(c)\right)+(1-\theta) \check{J}_{k}^{b}\left(\check{\mathbf{h}}_{k}(c)\right)=\theta\left[(1-a) J_{k}^{\mathbb{L}}\left(\check{\mathbf{h}}_{k}(c)\right)+a f\left(\check{\mathbf{h}}_{k}(c)\right)\right]+(1-$ $\theta)\left[(1-b) J_{k}^{\mathbb{L}}\left(\check{\mathbf{h}}_{k}(c)\right)+b f\left(\check{\mathbf{h}}_{k}(c)\right)\right]=[\theta(1-a)+(1-\theta)(1-b)] J_{k}^{\mathbb{L}}\left(\check{\mathbf{h}}_{k}(c)\right)+$ $[\theta a+(1-\theta) b] f\left(\check{\mathbf{h}}_{k}(c)\right)=(1-c) J_{k}^{\mathbb{L}}\left(\check{\mathbf{h}}_{k}(c)\right)+c f\left(\check{\mathbf{h}}_{k}(c)\right)=\check{J}_{k}^{c}\left(\check{\mathbf{h}}_{k}(c)\right)=g(c)$. Portanto, fica demonstrado que $g($.$) é função côncava.$

Além do fato de $g(\rho)$ ser côncava, é possível verificar que, quando $\rho=0$ (o que corresponde a $\gamma=0$ ), tem-se que $\check{\mathbf{h}}_{k}(0)=\hat{\mathbf{h}}_{k}$ e $g(0)=J_{k}(\mathbb{L})$; por outro lado, para o caso particular em que $f(\mathbf{x})=\|\mathbf{x}\|_{1}$, quando $\rho=1$ (o que corresponde a $\gamma=\infty)$, tem-se que $\check{\mathbf{h}}_{k}(1)=\mathbf{0}$ e $g(1)=f(\mathbf{0})=0$.

Além disso, é possível demonstrar o seguinte teorema:

Teorema 4.5 As funções $p(\rho)$ e $q(\rho)$ são, respectivamente, monótona crescente e monótona decrescente.

Prova. Combinando 4-72, 4-75, 4-76 e 4-77, tem-se que

$$
g(\rho)=(1-\rho) p(\rho)+\rho q(\rho)
$$

Admitindo-se que $p(\rho)$ e $q(\rho)$ são continuamente diferenciáveis (de classe $C^{1}$ ), derivando a expressão em 4-78 em relação a $\rho$, obtém-se

$$
g^{\prime}(\rho)=(1-\rho) p^{\prime}(\rho)+\rho q^{\prime}(\rho)+q(\rho)-p(\rho)
$$

Em consequência de 4-73, tem-se que, para $\rho$ qualquer, 


$$
\nabla \check{J}_{k}^{\rho}\left(\check{\mathbf{h}}_{k}(\rho)\right)=\nabla_{\mathbf{x}} \check{J}_{k}\left(\check{\mathbf{h}}_{k}(\rho), \rho\right)=\mathbf{0}
$$

Utilizando 4-72, a equação 4-80 pode ser reescrita da seguinte forma

$$
\nabla \check{J}_{k}^{\rho}\left(\check{\mathbf{h}}_{k}(\rho)\right)=(1-\rho) \nabla J_{k}^{\mathbb{L}}\left(\check{\mathbf{h}}_{k}(\rho)\right)+\rho \nabla f\left(\check{\mathbf{h}}_{k}(\rho)\right)=\mathbf{0}
$$

Seja $\check{\mathbf{h}}_{k}^{\prime}(\rho)=\left[h_{1}^{\prime}(\rho) h_{2}^{\prime}(\rho) \ldots h_{L}^{\prime}(\rho)\right]^{T}$, em que $h_{i}^{\prime}(\rho)$ é a derivada em relação a $\rho$ da i-ésima componente de $\check{\mathbf{h}}_{k}(\rho)$. Pela Regra da Cadeia, tem-se que

$$
\begin{aligned}
& p^{\prime}(\rho)=\Re\left\{\left[\nabla J_{k}^{\mathbb{L}}\left(\check{\mathbf{h}}_{k}(\rho)\right)\right]^{H} \check{\mathbf{h}}_{k}^{\prime}(\rho)\right\} \\
& q^{\prime}(\rho)=\Re\left\{\left[\nabla f\left(\check{\mathbf{h}}_{k}(\rho)\right)\right]^{H} \check{\mathbf{h}}_{k}^{\prime}(\rho)\right\}
\end{aligned}
$$

Combinando 4-81, 4-82 e 4-83, resulta que

$$
(1-\rho) p^{\prime}(\rho)+\rho q^{\prime}(\rho)=0
$$

$e$, portanto,

$$
\frac{p^{\prime}(\rho)}{q^{\prime}(\rho)}=-\frac{\rho}{1-\rho}
$$

Para $0<\rho<1$, como $\rho /(1-\rho)>0$, a equação 4-85 estabelece que, se $p^{\prime}(\rho)>0$, então $q^{\prime}(\rho)<0$, e vice-versa. Aplicando 4-84 em 4-79, tem-se que

$$
g^{\prime}(\rho)=q(\rho)-p(\rho)
$$

Derivando 4-86 em relação a $\rho$, e levando em consideração que a função $g(\rho)$ é côncava (Teorema 4.4), resulta que

$$
g^{\prime \prime}(\rho)=q^{\prime}(\rho)-p^{\prime}(\rho) \leq 0
$$

Finalmente, combinando 4-85 e 4-87, tem-se que $p^{\prime}(\rho) \geq 0$ e $q^{\prime}(\rho) \leq 0$ para todo $\rho \in[0,1]$. Portanto, fica demonstrado que $p(\rho)$ é função monótona crescente e $q(\rho)$ é função monótona decrescente ${ }^{3}$.

Devido à monotonicidade de $p(\rho)$ (ou de $q(\rho))$, tem-se como corolário,

$$
\rho_{1} \neq \rho_{2} \Leftrightarrow \check{\mathbf{h}}_{k}\left(\rho_{1}\right) \neq \check{\mathbf{h}}_{k}\left(\rho_{2}\right)
$$

Como a variação de $p(\rho)$ em função de $\rho$ ocorre no sentido oposto ao da variação de $q(\rho)$, ocorre que a variação de $\rho$ ao longo do intervalo [0,1] descreve uma curva de relação custo-benefício, (curva de tradeoff) entre essas duas funções.

${ }^{3}$ Admite-se que tanto $p($.$) quanto q($.$) não são funções constantes.$ 
A Figura 4.40 ilustra uma curva de tradeoffentre $p(\rho)$ e $q(\rho)$ para uma única realização de um sistema $\operatorname{MESS}(100,10), \operatorname{SINR}=10 \mathrm{~dB}, \operatorname{com} N=32$ e $k=50$, $\operatorname{com} p(\rho)=J_{k}^{\mathbb{L}}\left(\check{\mathbf{h}}_{k}(\rho)\right)$ e $q(\rho)=\left\|\check{\mathbf{h}}_{k}(\rho)\right\|_{1}$.
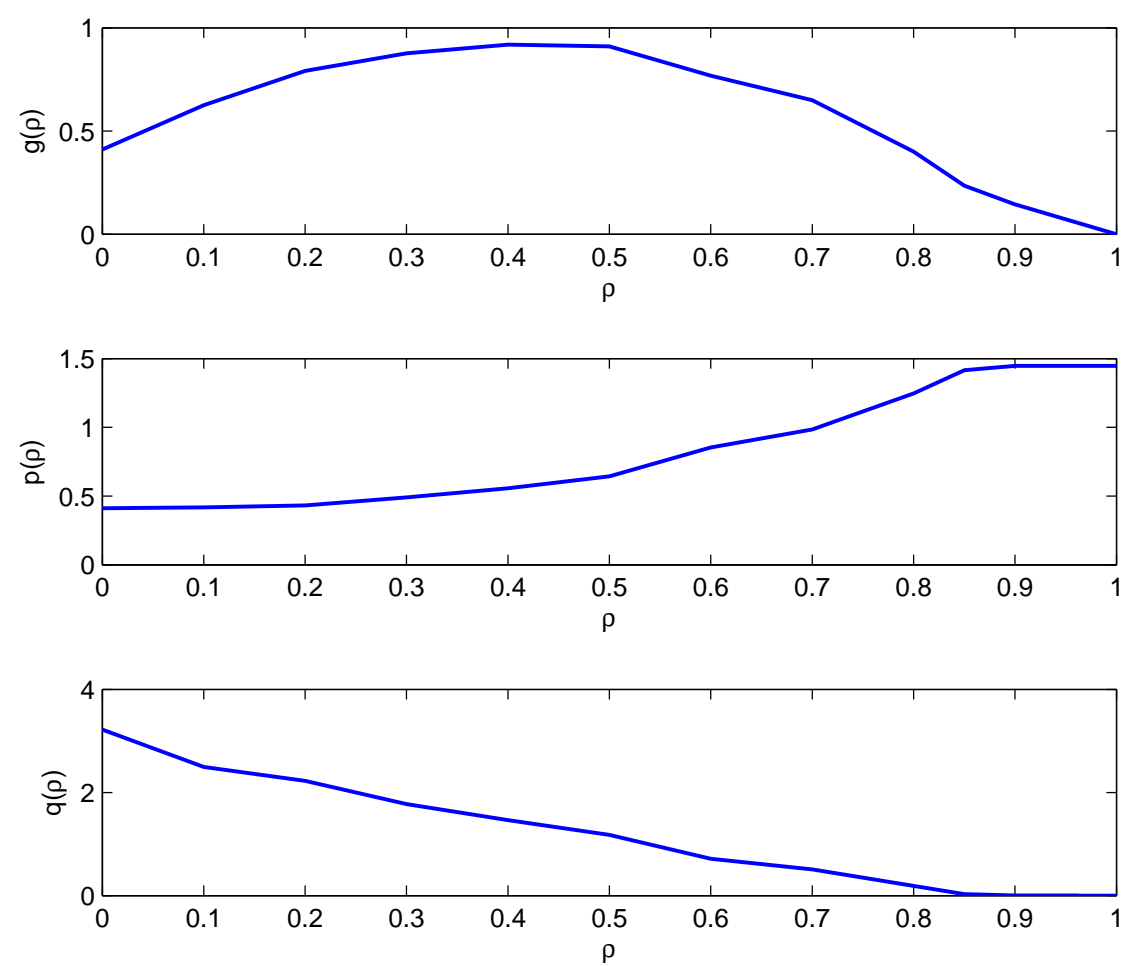

Figura 4.40: Curva de tradeoff entre $p(\rho)$ e $q(\rho)$ para uma única realização de um sistema $\operatorname{MESS}(100,10)$ e $\operatorname{SINR}=10 \mathrm{~dB}, \operatorname{com} N=32$ e $k=50$

\subsection{5}

\section{Algoritmo proposto}

A relação de equivalência estabelecida em 4-74 e a correspondência biunívoca entre $\gamma$ e $\rho$ possibilitam a obtenção de $\mathbf{h}_{k}^{*}$ a partir de $\rho^{*}$ em vez de $\gamma^{*}$. A busca por $\rho^{*}$ é mais vantajosa que a busca por $\gamma^{*}$, haja vista que $\rho$ pertence ao intervalo limitado $[0,1]$.

O algoritmo aqui proposto de Regularização Automática do custo LS, descrito no Algoritmo 4.9, tem como propósito a obtenção de $\mathbf{h}_{k}^{*}$ ao mesmo tempo em que se busca $\rho^{*}$ tal que $p\left(\rho^{*}\right)=J_{\max }$. O parâmetro tol corresponde à tolerância admitida ao erro $\left|p(\rho)-J_{\max }\right| / J_{\max }$. O parâmetro $\lambda$ corresponde ao fator de relaxamento do custo LS, para determinação de $J_{\max }$. 
O algoritmo de Regularização Automática do custo LS baseia-se no método de bisseção para a busca de $\rho^{*}$. Iniciando com $\rho_{\min }^{(0)}=0, \rho_{\max }^{(0)}=1$ e $\rho^{(0)}=0,5$, o método de bisseção consiste em, a cada nova iteração $i$-ésima iteração, fazer o seguinte: se $p\left(\rho^{(i-1)}\right)<J_{\max }$, então $\rho_{\min }^{(i)} \leftarrow \rho^{(i-1)}, \rho_{\max }^{(i)} \leftarrow \rho_{\max }^{(i-1)}$ e $\rho^{(i)} \leftarrow\left[\rho_{\min }^{(i)}+\rho_{\max }^{(i)}\right] / 2 ;$ caso contrário, $\rho_{\max }^{(i)} \leftarrow \rho^{(i-1)}, \rho_{\min }^{(i)} \leftarrow \rho_{\min }^{(i-1)}$ e $\rho^{(i)} \leftarrow$ $\left[\rho_{\min }^{(i)}+\rho_{\max }^{(i)}\right] / 2$. O método de bisseção fundamenta-se no fato de que $p(\rho)$ é monótona crescente. As iterações se repetem até que $\left|p\left(\rho^{(i)}\right)-J_{\max }\right| / J_{\max }<t o l$, quando é feito $\rho^{*} \leftarrow \rho^{(i)}$.

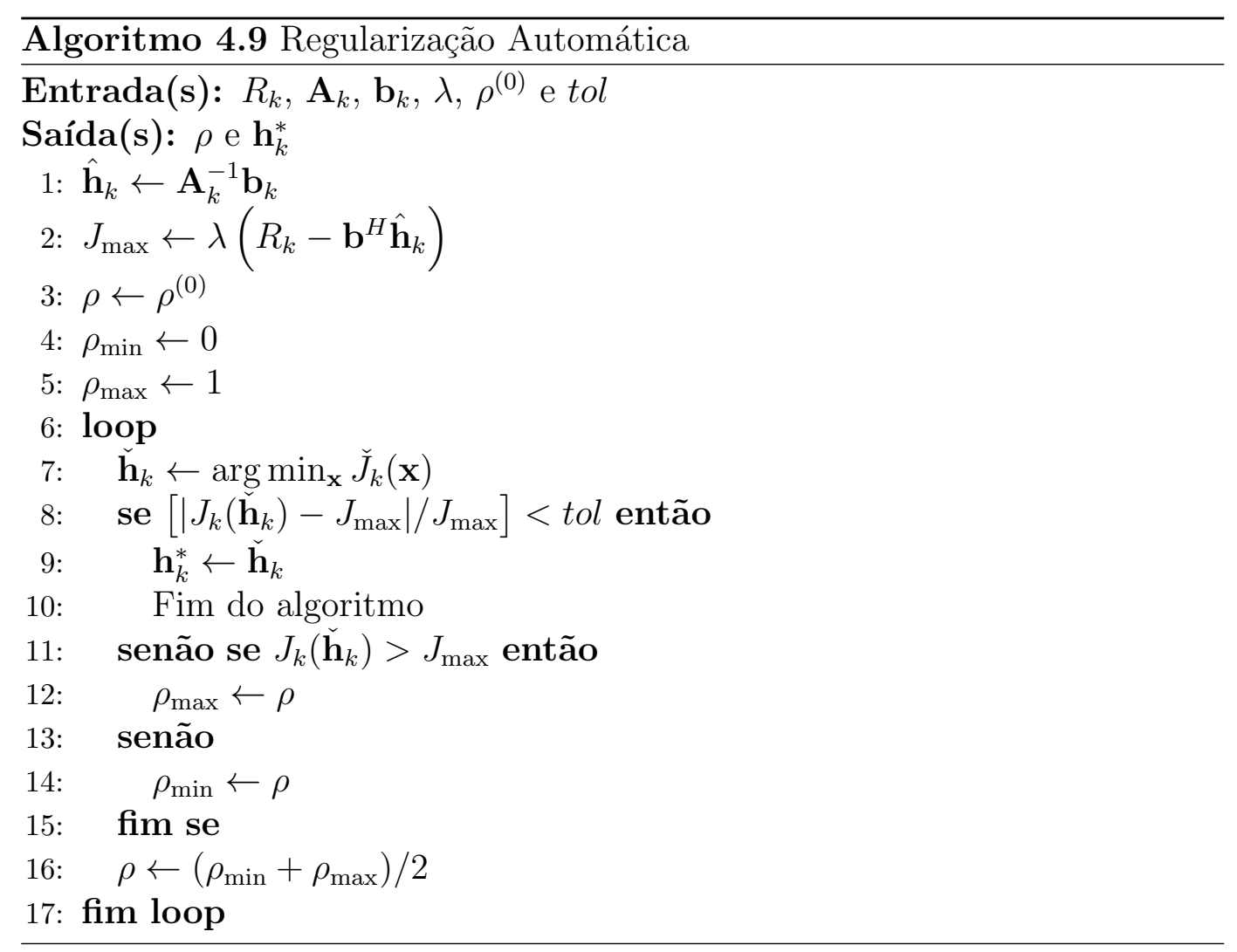

\subsection{6}

\section{Algoritmo de busca para obtenção da estimativa regularizada}

O passo 7 do Algoritmo 4.9 corresponde à obtenção de $\check{\mathbf{h}}_{k}(\rho)$. Nesta seção, propõe-se um algoritmo de busca para obtenção de $\check{\mathbf{h}}_{k}(\rho)$ baseado em backtracking line search, tendo como direção de busca o oposto do gradiente do custo LS regularizado, para o caso particular em que a função de regularização é dada por

$$
f(\mathbf{x})=\|\mathbf{x}\|_{1}
$$

Nesse caso, o gradiente da função de regularização é dado por 


$$
\nabla f(\mathbf{x})=\operatorname{sgn}(\mathbf{x})
$$

onde $\operatorname{sgn}(\mathbf{x})=\left[\operatorname{sgn}\left(x_{1}\right) \operatorname{sgn}\left(x_{2}\right) \ldots \operatorname{sgn}\left(x_{L}\right)\right]^{T}$, e $\operatorname{sgn}(z): \mathbb{C} \rightarrow \mathbb{C}$ é dada por

$$
\operatorname{sgn}(z)=\left\{\begin{array}{cc}
\frac{z}{|z|} & z \neq 0 \\
0 & z=0
\end{array}\right.
$$

O gradiente de $\breve{J}_{k}^{\rho}(\mathbf{x})$ é dado por

$$
\nabla \check{J}_{k}^{\rho}(\mathbf{x})=(1-\rho) \nabla J_{k}^{\mathbb{L}}(\mathbf{x})+\rho \nabla f(\mathbf{x})
$$

Empregando 3-8 e 4-90, obtém-se o gradiente de $\breve{J}_{k}^{\rho}(\mathbf{x})$, que é dado por

$$
\nabla \check{J}_{k}^{\rho}(\mathbf{x})=2(1-\rho)\left[\mathbf{A}_{k} \mathbf{x}-\mathbf{b}_{k}\right]+\rho \times \operatorname{sgn}(\mathbf{x})
$$

Para acelerar a velocidade de convergência, o algoritmo de busca aqui proposto incorpora um mecanismo opcional de hard-tresholding, cujo valor de corte é dado pelo parâmetro de entrada $\eta$. Este mecanismo não restringe o espaço de buscas, haja vista que, em cada iteração do algoritmo, os elementos zerados de $\tilde{\mathbf{h}}_{k}$ podem novamente ultrapassar o limiar $\eta$ de hard-thresholding.

Outra característica do algoritmo de busca proposto nesta seção é que ele realiza a normalização do gradiente antes de executar a busca pelo método de backtracking line search, o que possibilita o cálculo do parâmetro de busca $\beta$ em função dos parâmetros de entrada maxiteracoes (que corresponde ao número máximo de iterações admitido por busca) e $\epsilon$ (valor mínimo admitido para norma do vetor de busca).

O Algoritmo 4.10 descreve em detalhes o algoritmo de busca proposto nesta seção, o qual pode ser empregado no passo 7 do Algoritmo de Regularização Automática (Algoritmo 4.9).

\subsection{7}

\section{Algoritmo de Regularização Automática em versão iterativa}

Todos os algoritmos de estimação de sistemas esparsos apresentados até o momento foram projetados para funcionar ao final da sequência de treinamento. Comparado com os métodos apresentados anteriormente, o método de regularização automática do custo LS possui a vantagem de poder ser implementado de forma iterativa, na qual o esforço computacional é distribuído ao longo da sequência de treinamento.

A diferença fundamental entre as versões não-iterativa e iterativa do algoritmo de regularização automática é que, enquanto na primeira versão há um loop 


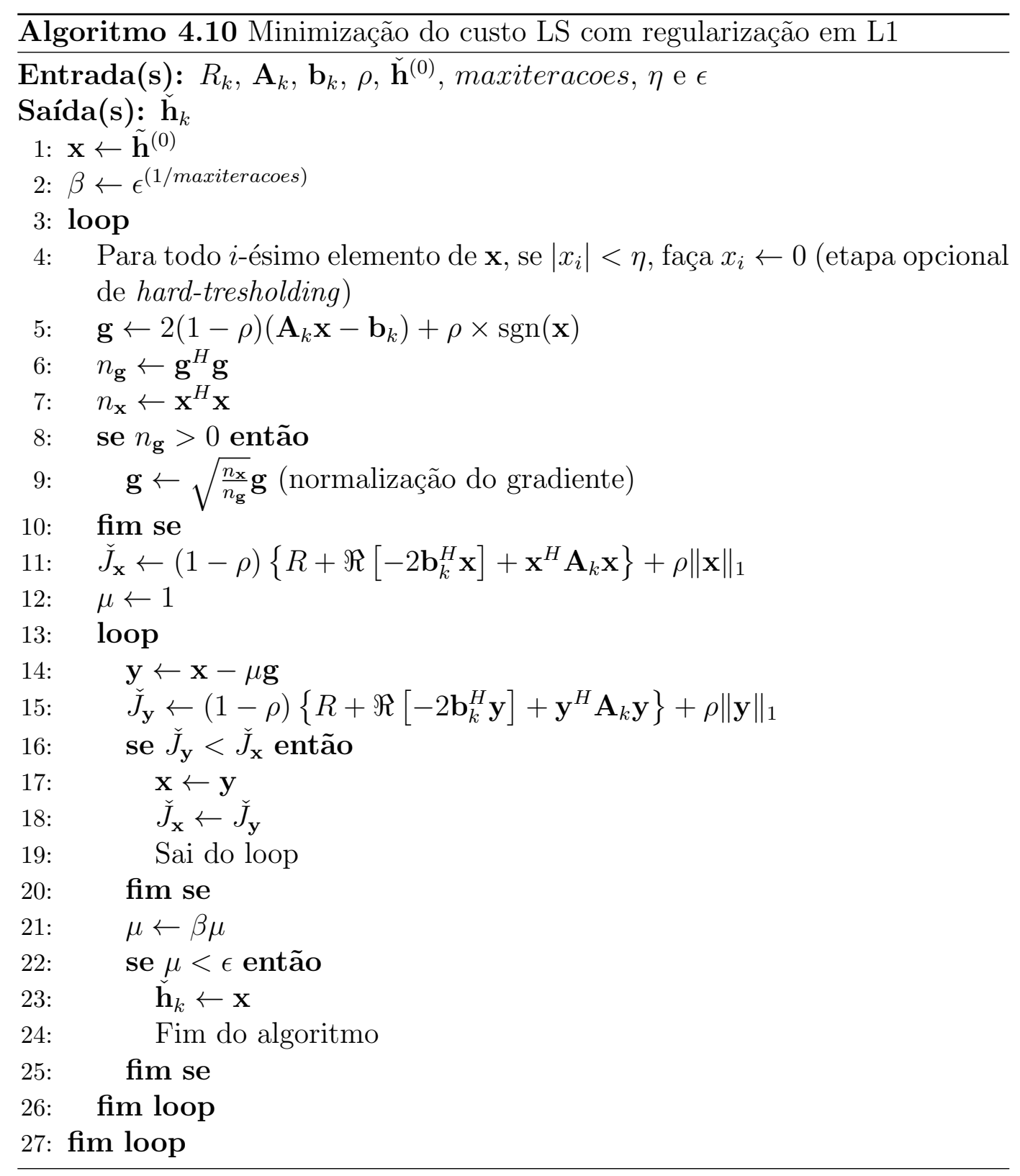

em que são realizadas várias iterações da bisseção do intervalo de busca até a obtenção de $\rho^{*}$ e $\check{\mathbf{h}}_{k}^{*}$, na segunda versão esse loop não existe, e a cada iteração do algoritmo é realizada apenas uma iteração da bisseção do intervalo de busca.

O algoritmo aqui proposto de Regularização Automática do Custo LS, em sua versão iterativa, é descrito no Algoritmo 4.11. É recomendável iniciar o algoritmo com $\check{\mathbf{h}}_{0}=\hat{\mathbf{h}}_{0}, \rho_{\max , 0}=1$ e $\rho_{\min , 0}=0$.

A Figura 4.41 ilustra a variação dos parâmetros $\rho_{\min }, \rho_{\max }$ e $\rho$ empregandose o algoritmo iterativo de regularização automática do custo LS (Algoritmo 4.11) ao longo da sequência de treinamento para uma única realização de um sistema $\operatorname{MESS}(100,10)$, com $\mathrm{SINR}=15 \mathrm{~dB}, N=16$ e $\lambda=1,05$. Os parâmetros iniciais do algoritmo foram: $\check{\mathbf{h}}_{0}=\hat{\mathbf{h}}_{1}, \rho_{\max , 0}=1$ e $\rho_{\min , 0}=1 E-10, \rho_{0}=0,5$, tol $=0,01, \eta=0,0001, \beta=0,8$ e $\epsilon=0,001$. Nela, é possível observar o 


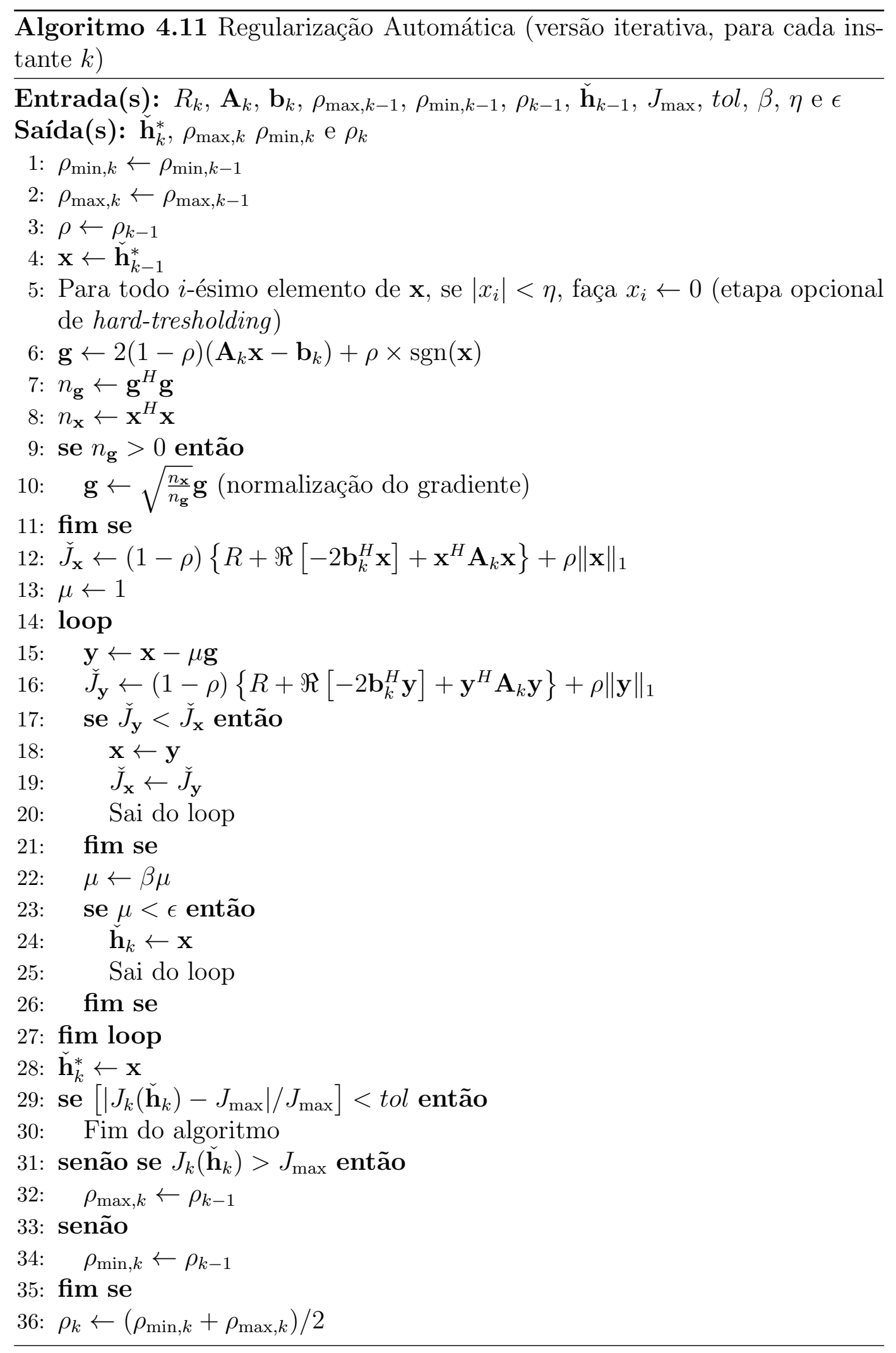


mecanismo de bisseção em funcionamento.

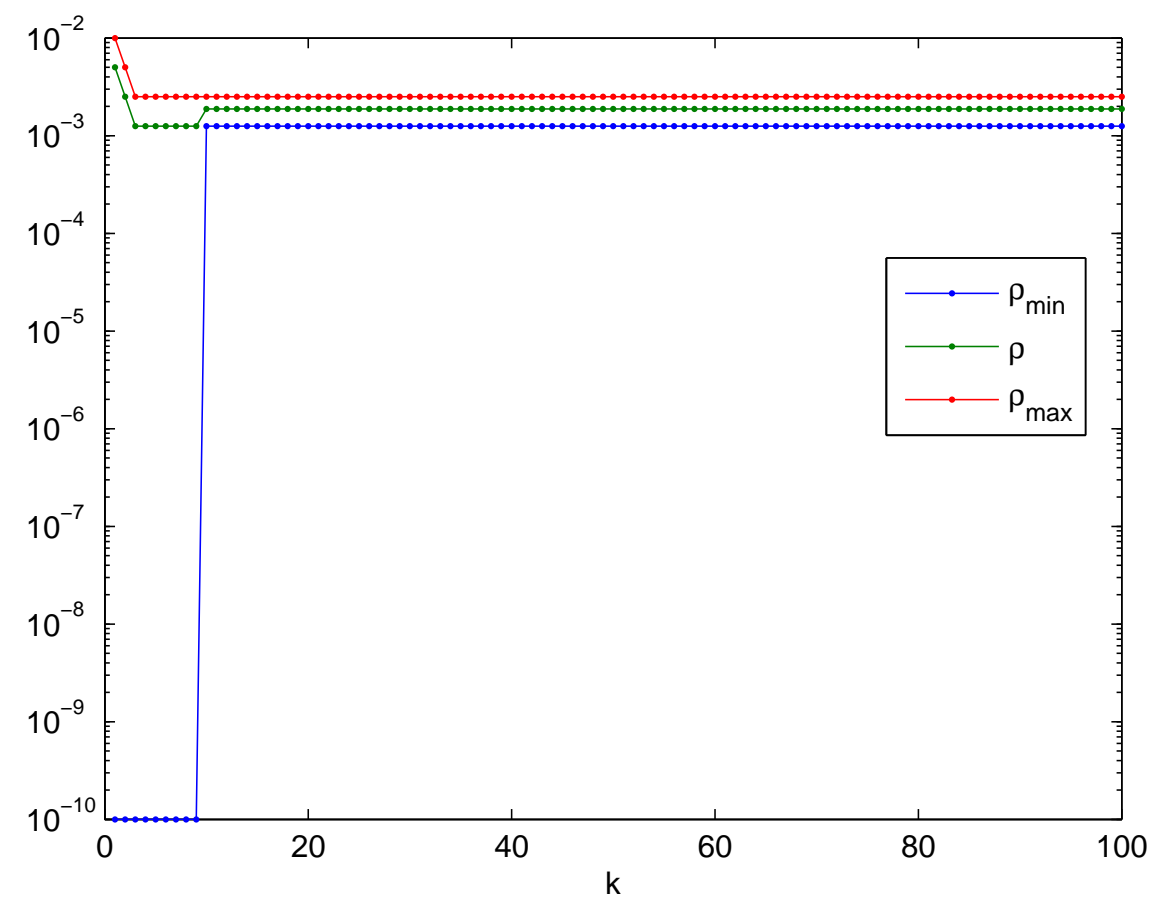

Figura 4.41: Mecanismo de bisseção em uma única realização de canal gaussiano esparso com $L=100, S=10, \mathrm{SINR}=15 \mathrm{~dB}, N=32$ e $\lambda=1,05$. Parâmetros iniciais: $\check{\mathbf{h}}_{0}=\hat{\mathbf{h}}_{1}, \rho_{\max , 0}=1$ e $\rho_{\min , 0}=1 E-10, \rho_{0}=0,01$, tol $=0,01, \eta=0,0001, \beta=0,8$ e $\epsilon=0,001$

A Figura 4.42 mostra a variação média do fator de regularização $\rho$ empregandose o algoritmo iterativo de regularização automática do custo LS (Algoritmo 4.11), estimada com $Q=1000$ realizações independentes de um sistema $\operatorname{MESS}(100, S)$, com $\lambda=1,05$, em quatro situações diferentes: (a) $S=10$ e $\mathrm{SINR}=10 \mathrm{~dB}$, (b) $S=10$ e $\mathrm{SINR}=25 \mathrm{~dB}$, (c) $S=20$ e $\mathrm{SINR}=$ $10 \mathrm{~dB}$ e, finalmente, (d) $S=25$ e SINR $=20 \mathrm{~dB}$. Nota-se que, para cada um dos cenários de simulação, $\rho$ converge para um valor diferente, em aproximadamente 10 iterações. 


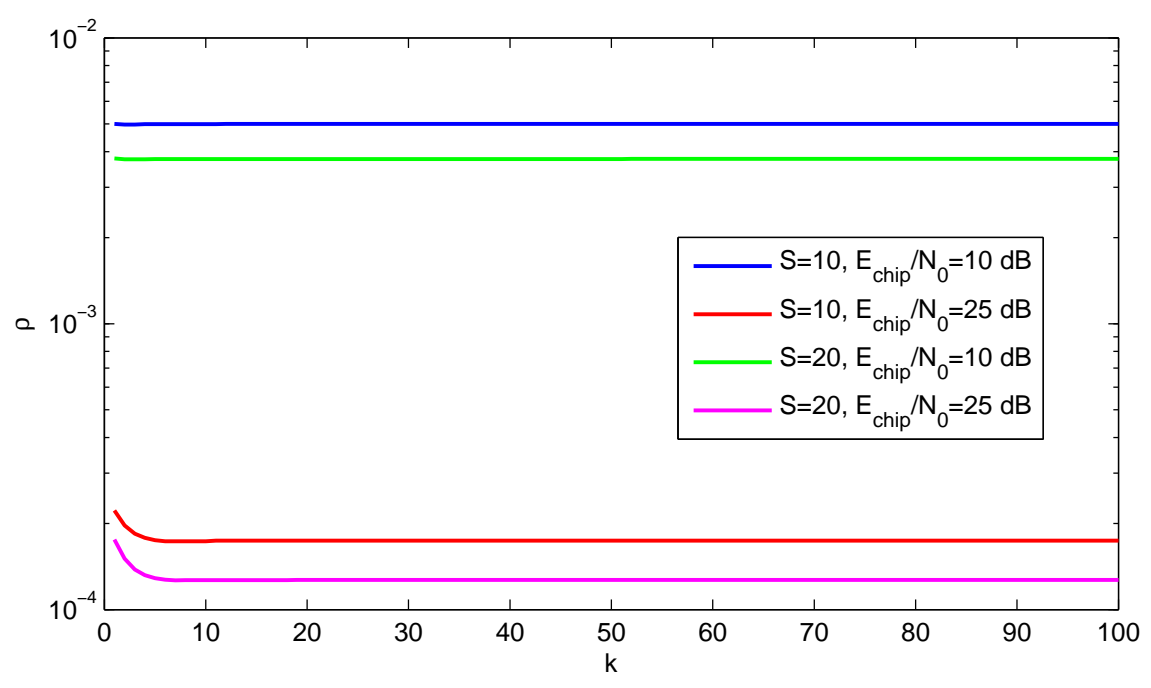

Figura 4.42: Variação média do fator de regularização $\rho$ ao longo da sequência de treinamento referente a $Q=1000$ realizações independentes de um sistema $\operatorname{MESS}(100, S), \operatorname{com} N=16$ e $\lambda=1,05$. Parâmetros iniciais: $\check{\mathbf{h}}_{0}=\hat{\mathbf{h}}_{1}$, $\rho_{\max , 0}=1$ e $\rho_{\min , 0}=1 E-10, \rho_{0}=0,5$, tol $=0,01, \eta=0,0001, \beta=0,8$ $\mathrm{e} \epsilon=0,001$

\subsection{8}

\section{Custo computacional}

O método de regularização automática do custo LS é o que apresenta o maior custo computacional dentre todos os métodos de estimação de sistemas esparsos apresentados nesta Tese, conforme mostram os resultados das simulações apresentados a seguir. Por outro lado, sua versão iterativa apresenta a vantagem, em relação aos demais métodos apresentados, de permitir a distribuição do custo computacional total ao longo de toda a sequência de treinamento.

\subsection{9}

\section{Análise de desempenho}

As Figuras 4.43, 4.44, 4.45 e 4.46 mostram o desempenho do algoritmo iterativo do método de regularização automática do custo LS (Algoritmo 4.11), comparado com o desempenho das estimações LS full-support e LS oráculo, ao longo da sequência de treinamento, com $\mathrm{N}=16, \lambda=1,05$, sistema $\operatorname{MESS}(100,10)$ e $\operatorname{SINR}=5,10,15,20,25$ e $30 \mathrm{~dB}$ respectivamente. As Figuras $4.49,4.50,4.51$ e 4.52 mostram o desempenho dos mesmos algoritmos na estimação de um sistema MESS(100,20), mantendo-se os demais parâmetros de simulação inalterados. Foram consideradas $Q=1000$ realizações independentes 
do sistema para cada valor de SINR. Em cada figura, nos gráficos superiores, são mostrados os desempenhos dos algoritmos citados em termos de NMSD e, nos gráficos inferiores, são mostradas as variações de $\rho_{\min }, \rho$ e $\rho_{\max }$ ao longo da sequência de treinamento.
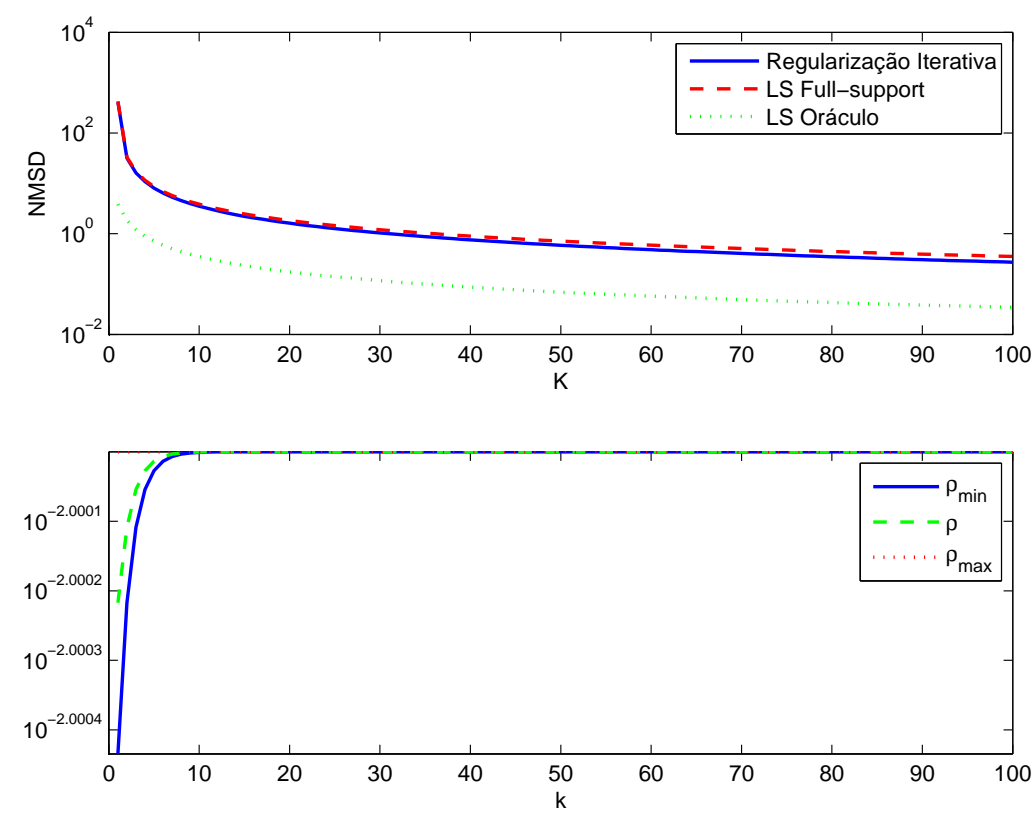

Figura 4.43: Reg. iter.: $\operatorname{MESS}(100,10), \operatorname{SINR}=5 \mathrm{~dB}, \lambda=1,05, \mathrm{~N}=16$ e $\mathrm{Q}=1000$
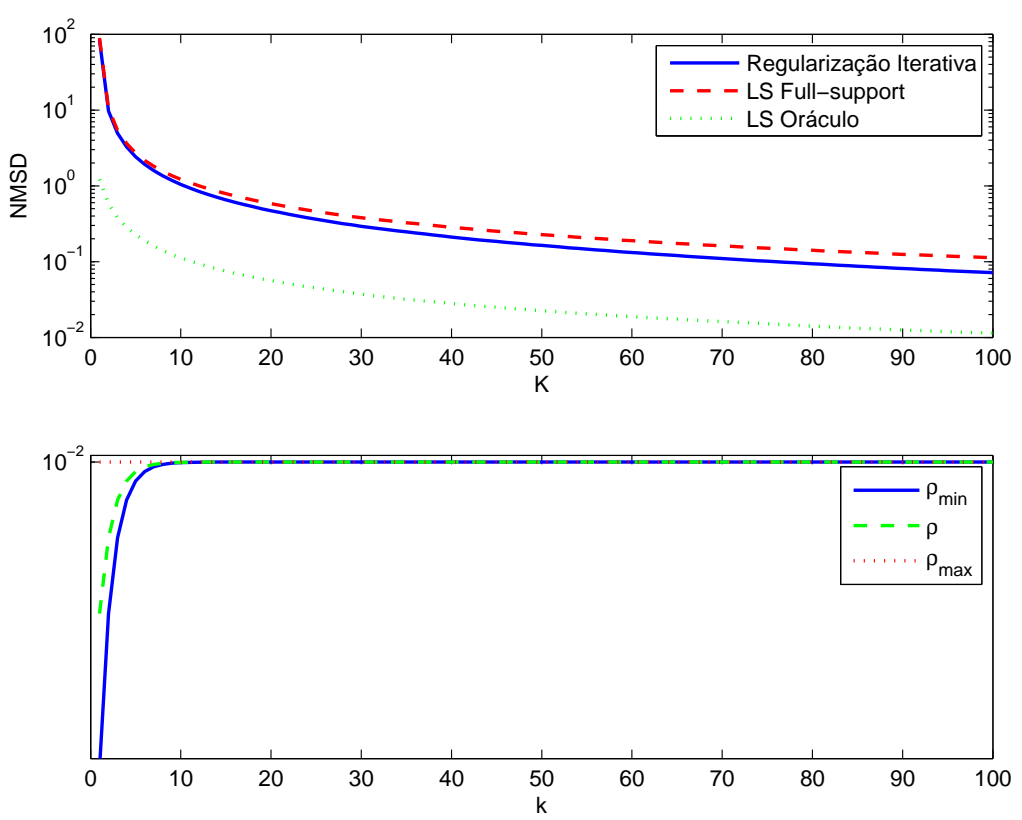

Figura 4.44: Reg. iter.: $\operatorname{MESS}(100,10), \mathrm{SINR}=10 \mathrm{~dB}, \lambda=1,05, \mathrm{~N}=16$ e $\mathrm{Q}=1000$ 

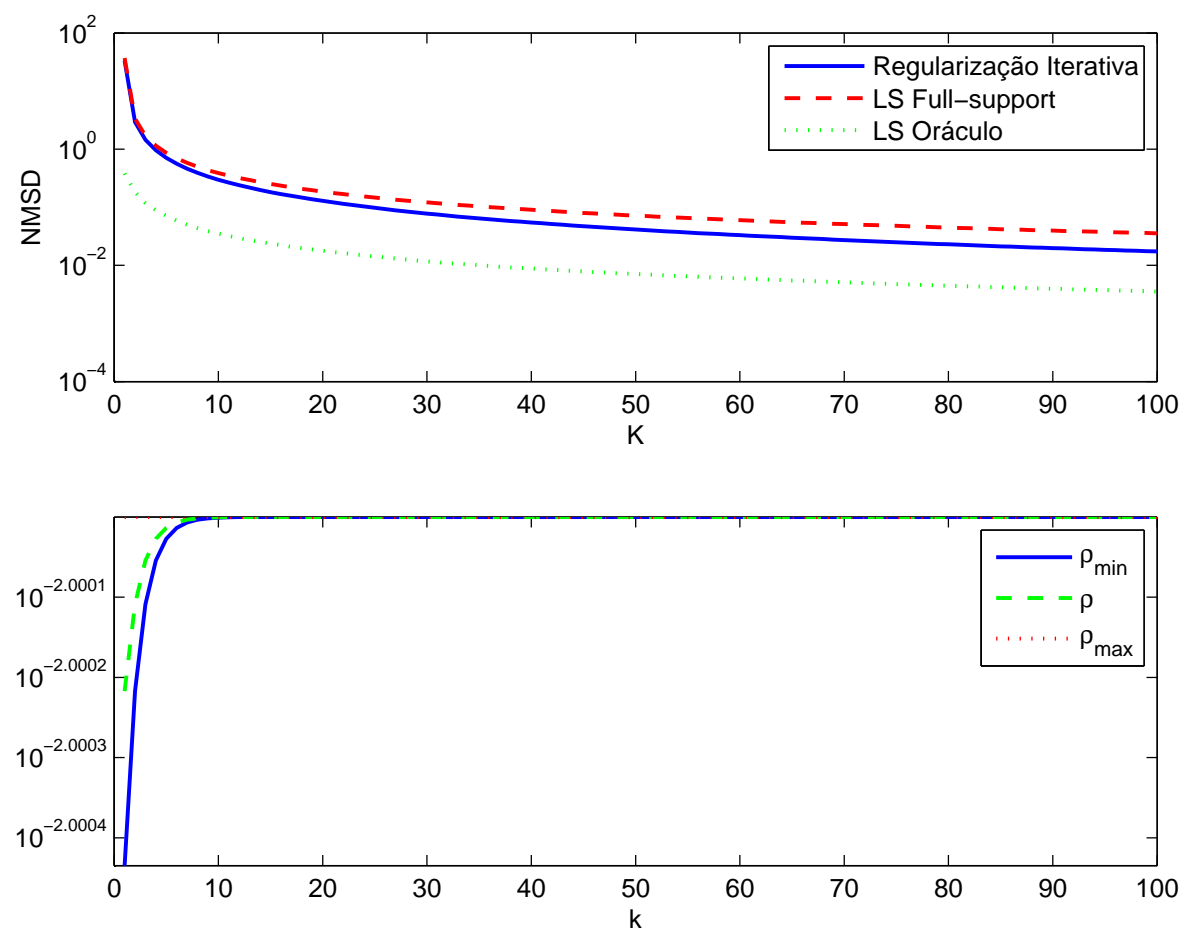

Figura 4.45: Reg. iter.: $\operatorname{MESS}(100,10), \mathrm{SINR}=15 \mathrm{~dB}, \lambda=1,05, \mathrm{~N}=16$ e $\mathrm{Q}=1000$
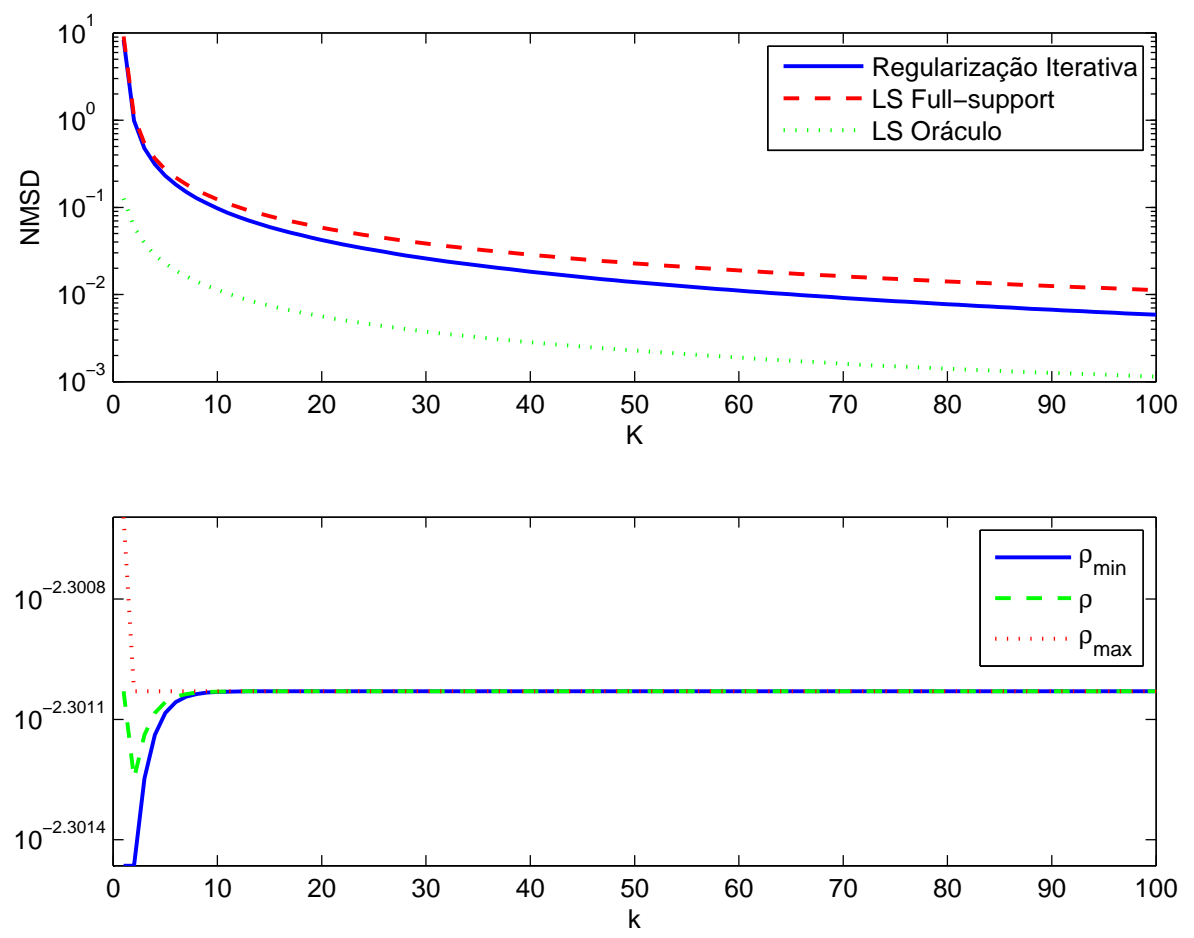

Figura 4.46: Reg. iter.: $\operatorname{MESS}(100,10), \operatorname{SINR}=20 \mathrm{~dB}, \lambda=1,05, \mathrm{~N}=16$ e $\mathrm{Q}=1000$ 

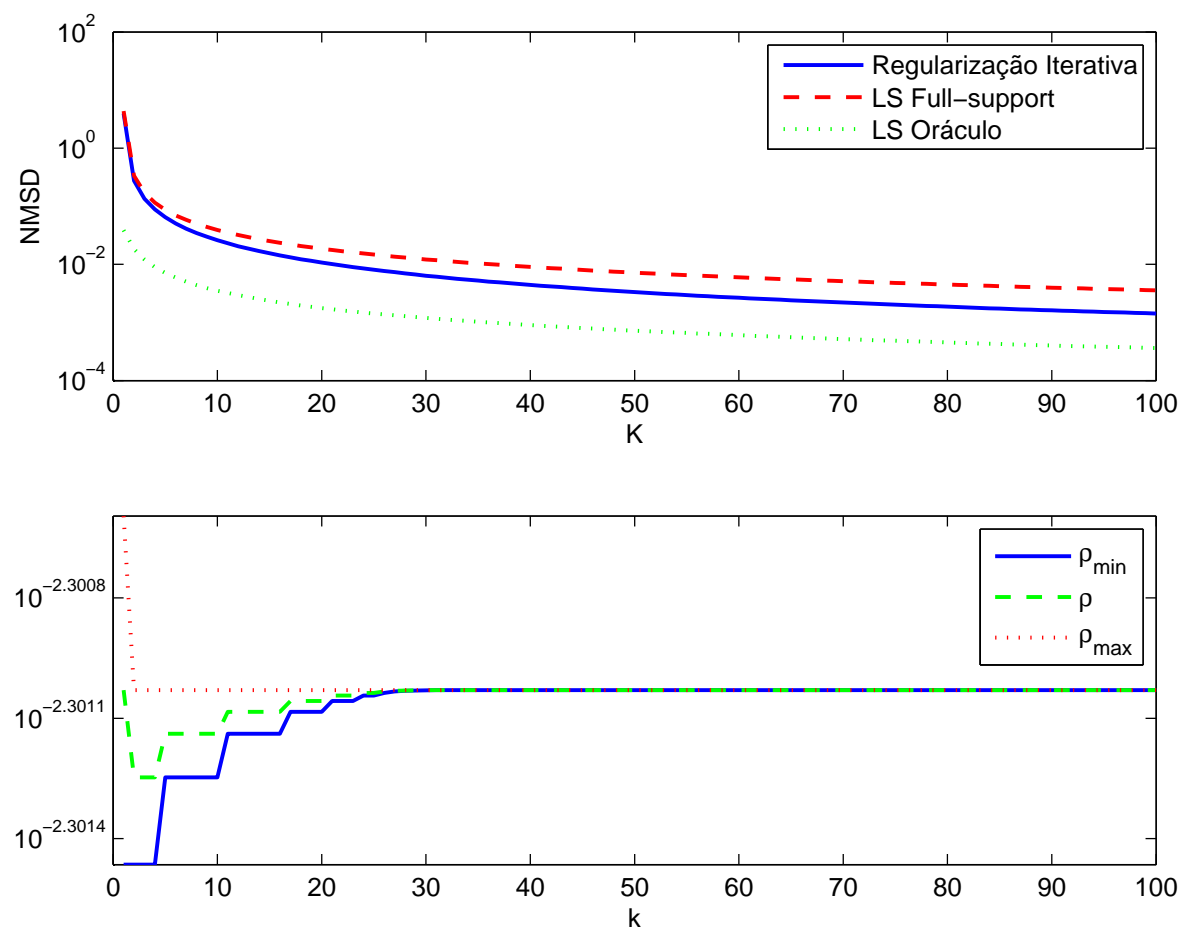

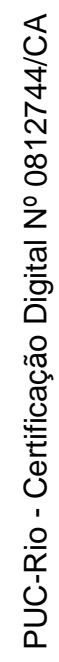

Figura 4.47: Reg. iter.: $\operatorname{MESS}(100,10), \mathrm{SINR}=25 \mathrm{~dB}, \lambda=1,05, \mathrm{~N}=16$ e $\mathrm{Q}=1000$
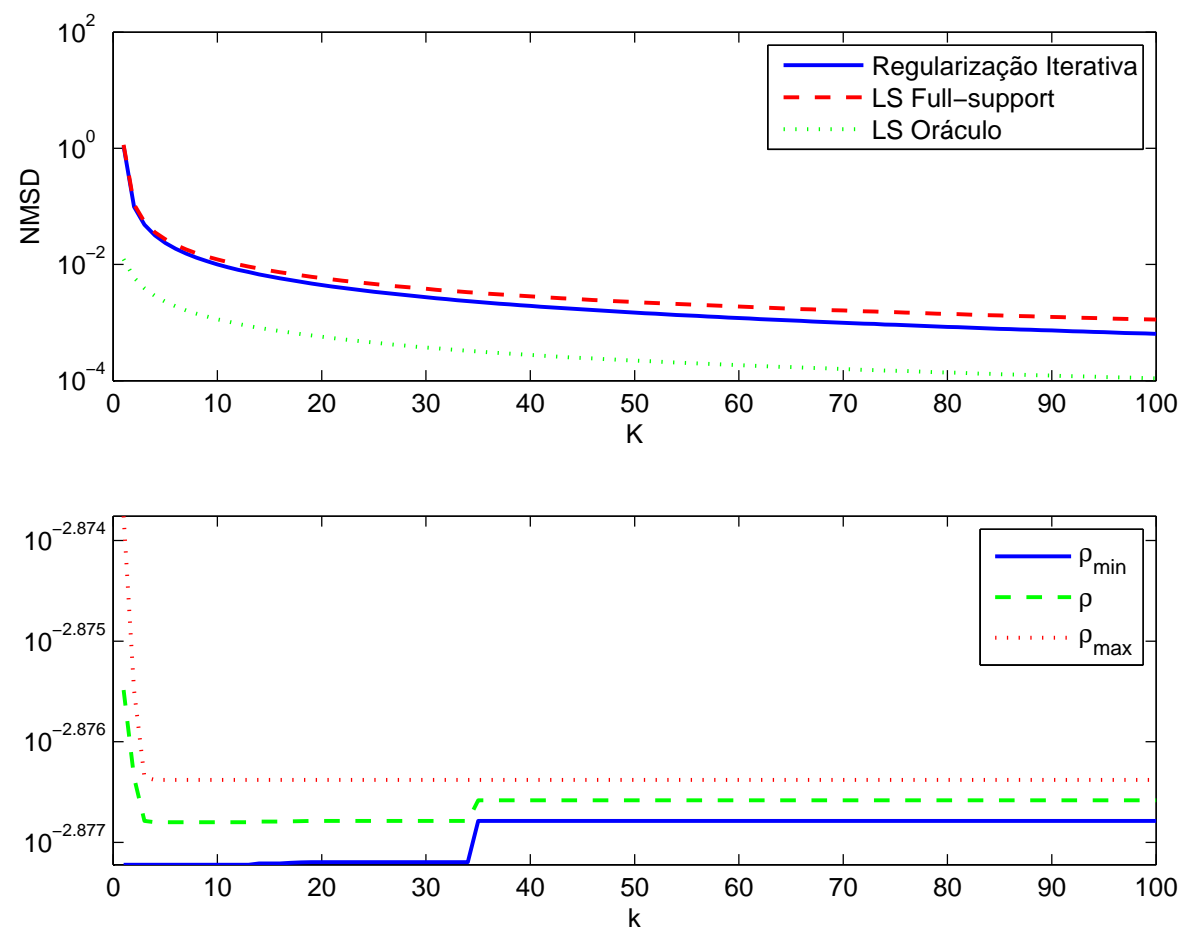

Figura 4.48: Reg. iter.: $\operatorname{MESS}(100,10), \mathrm{SINR}=30 \mathrm{~dB}, \lambda=1,05, \mathrm{~N}=16$ e $\mathrm{Q}=1000$ 

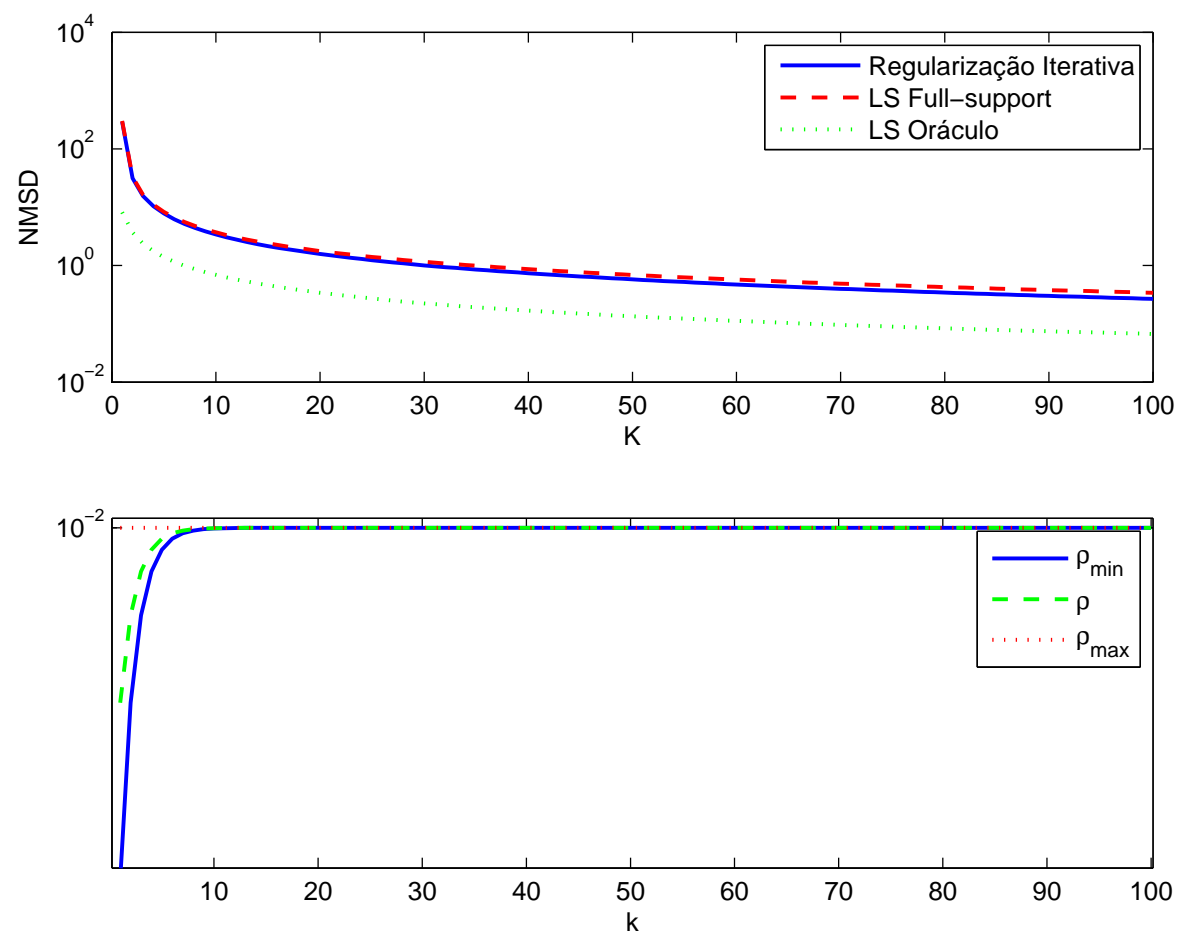

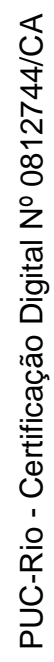

Figura 4.49: Reg. iter.: $\operatorname{MESS}(100,20), \operatorname{SINR}=5 \mathrm{~dB}, \lambda=1,05, \mathrm{~N}=16$ e $\mathrm{Q}=1000$
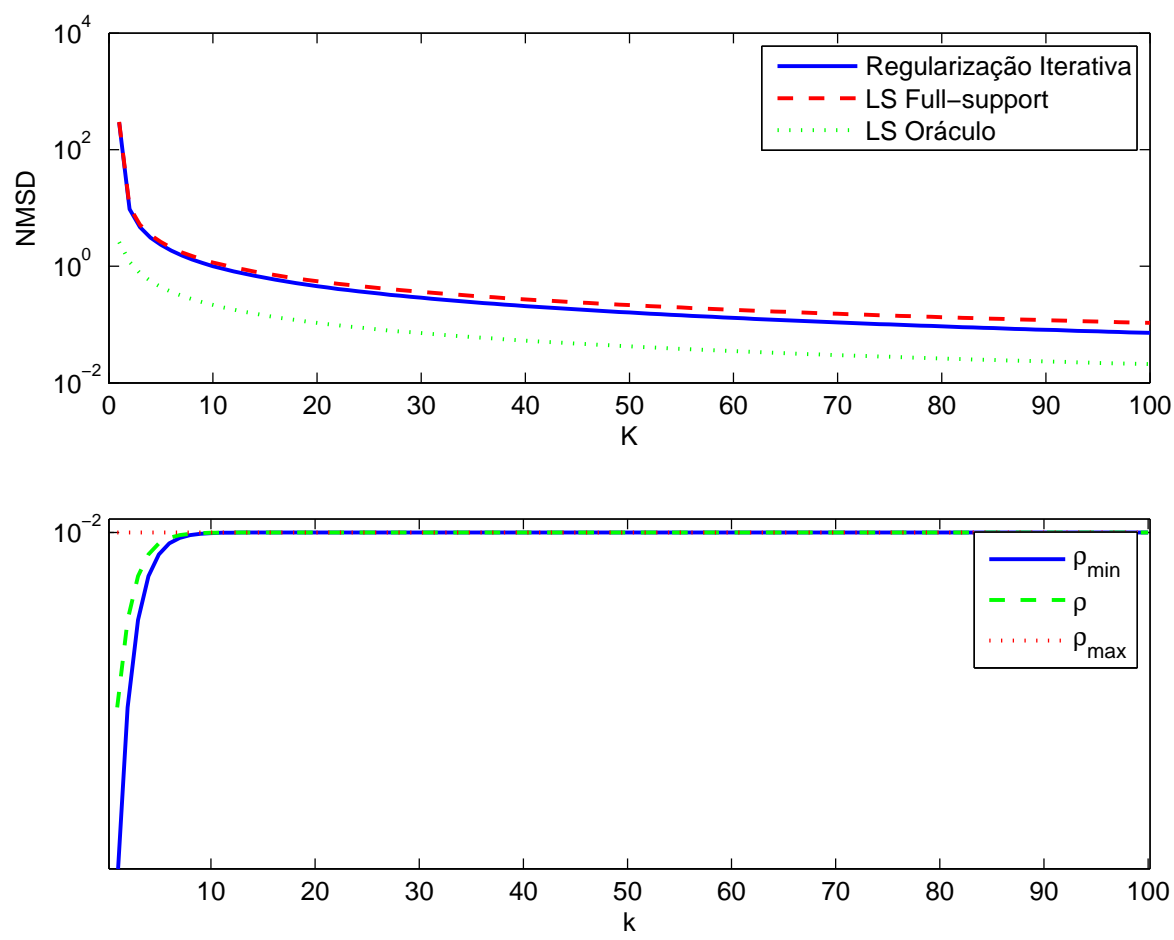

Figura 4.50: Reg. iter.: $\operatorname{MESS}(100,20), \mathrm{SINR}=10 \mathrm{~dB}, \lambda=1,05, \mathrm{~N}=16$ e $\mathrm{Q}=1000$ 

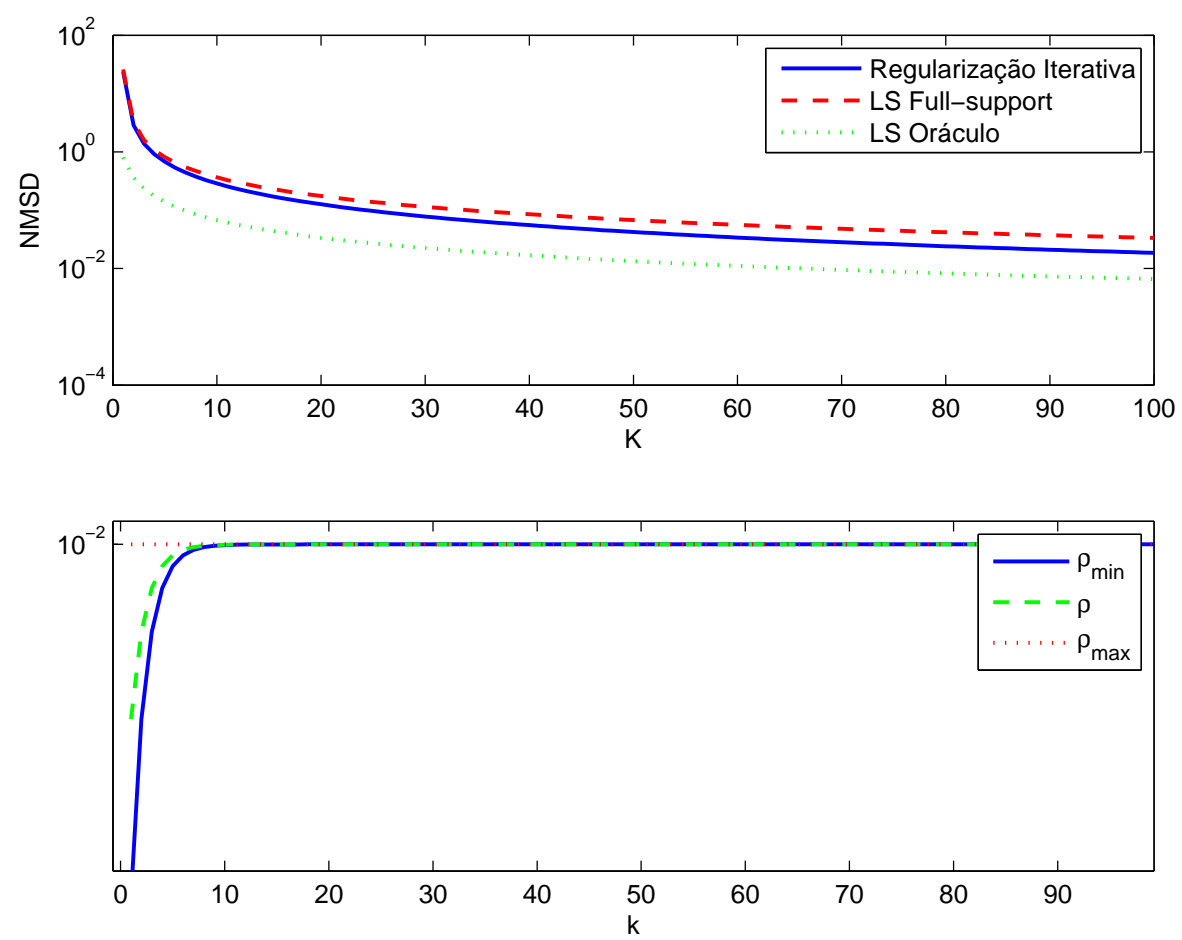

Figura 4.51: Reg. iter.: $\operatorname{MESS}(100,20), \operatorname{SINR}=15 \mathrm{~dB}, \lambda=1,05, \mathrm{~N}=16$ e $\mathrm{Q}=1000$
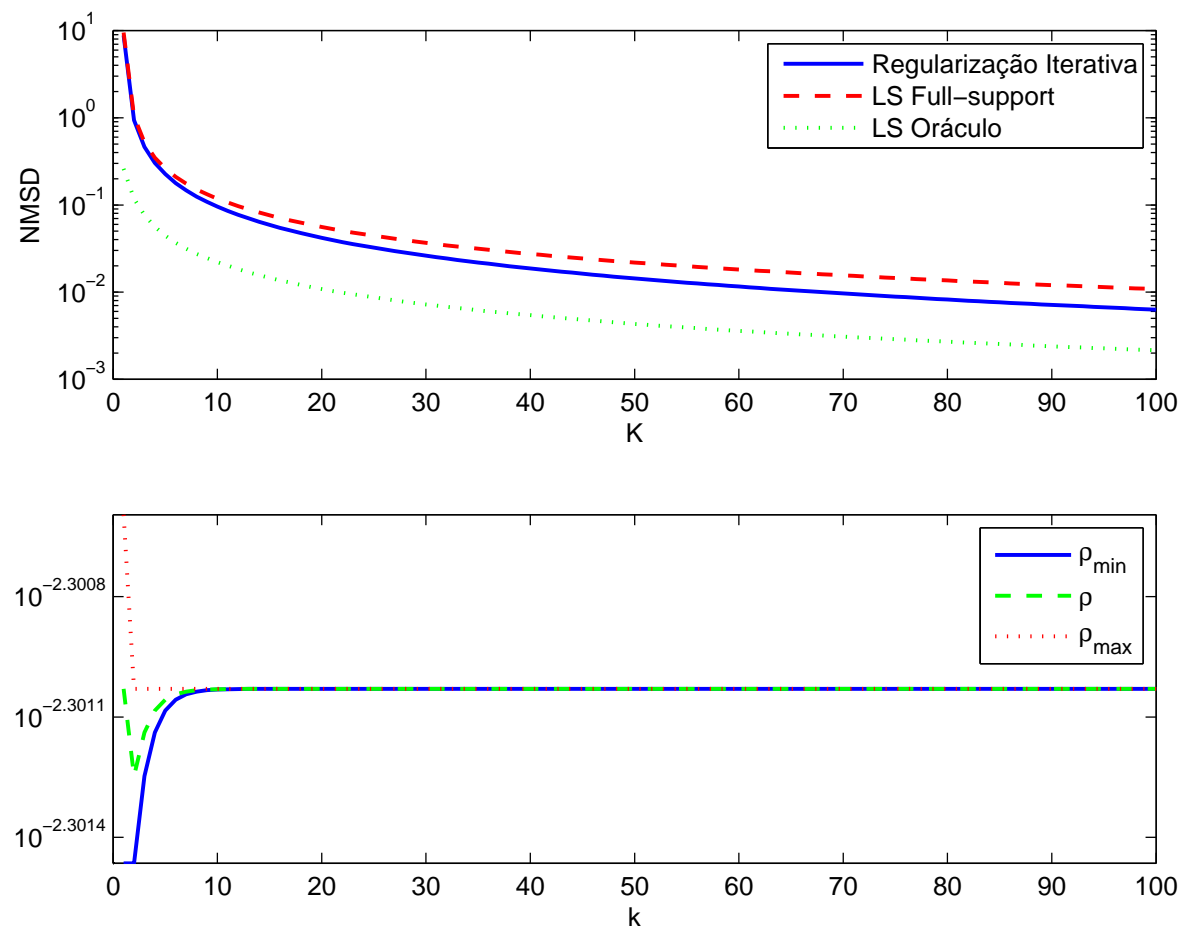

Figura 4.52: Reg. iter.: $\operatorname{MESS}(100,20), \mathrm{SINR}=20 \mathrm{~dB}, \lambda=1,05, \mathrm{~N}=16$ e $\mathrm{Q}=1000$ 

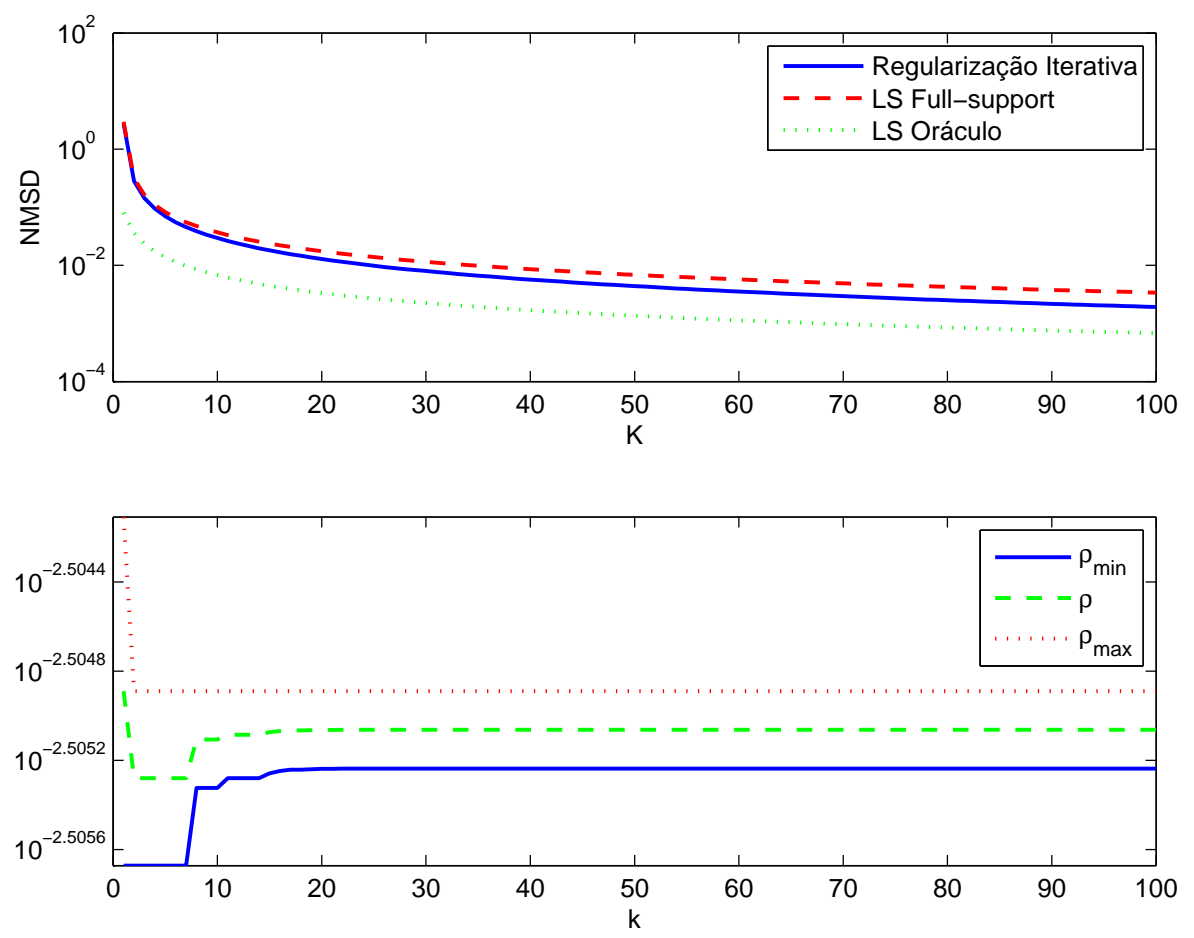

Figura 4.53: Reg. iter.: $\operatorname{MESS}(100,20), \operatorname{SINR}=25 \mathrm{~dB}, \lambda=1,05, \mathrm{~N}=16$ e $\mathrm{Q}=1000$
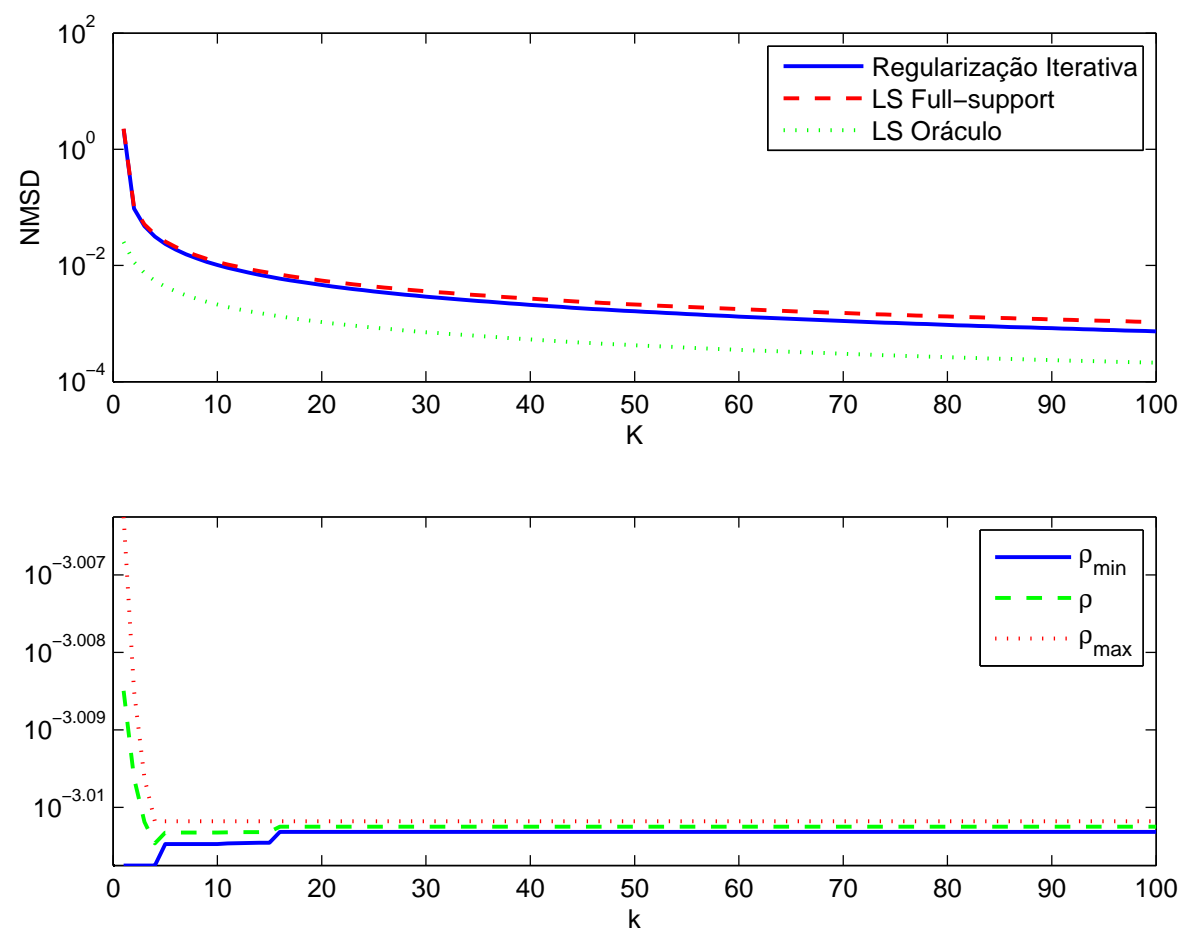

Figura 4.54: Reg. iter.: $\operatorname{MESS}(100,20), \mathrm{SINR}=30 \mathrm{~dB}, \lambda=1,05, \mathrm{~N}=16$ e $\mathrm{Q}=1000$ 
As figuras mostram que, dependendo de SINR e de $S$, o valor do fator de regularização converge para resultados muito diferentes; adicionalmente, os resultados mostram que, o valor do fator de regularização for fixo para todas as amostras geradas de resposta ao impulso do sistema, o desempenho da estimação LS com regularização é consideravelmente degradado. Esses dois fatores demonstram a utilidade do método proposto de ajuste automático do fator de regularização. Além disso, é possível verificar que as curvas de desempenho do algoritmo de Regularização Automática mantém-se sempre entre as curvas do LS Full-support e do LS Oráculo, o que demonstra a sua robustez.

\section{9}

\section{Considerações finais}

Neste capítulo, foram originalmente elaborados e apresentados quatro métodos projetados especificamente para a estimação de sistemas esparsos. São eles:

- Encolhimentos Sucessivos;

- Expansões Sucessivas;

- Minimização da norma $l_{1}$;

- Regularização do Custo LS.

Buscou-se aqui realizar a apresentação dos métodos citados de maneira unificada, em que o elo de ligação entre eles é o parâmetro $\lambda$ (fator de relaxamento do custo LS), que regula o custo LS máximo admitido $J_{\max }$. Por isso, alguns critérios para escolha do máximo valor admitido para o custo LS, baseados nas equações de convergência do custo LS apresentadas no capítulo anterior, foram apresentados.

Em seguida, foi apresentada a função $L_{0}($.$) como uma espécie de aproximação$ contínua e diferenciável da pseudo-norma $l_{0}$. Através desta função, mostrouse o vínculo existente entre a pseudo-norma $l_{0}$ e a norma $l_{1}$, no intuito de esclarecer o fato de a norma $l_{1}$ ser frequentemente empregada na técnicas de estimação esparsas encontradas na literatura. Além disso, a função $L_{0}($. revelou-se uma ferramenta útil para estimação da cardinalidade do suporte da resposta ao impulso do canal, tendo sido empregada em uma versão do algoritmo de Encolhimentos Sucessivos com limitação da cardinalidade mínima do suporte da resposta ao impulso do canal estimado.

Numa introdução aos métodos de estimação previamente citados, a tarefa de estimação ótima do suporte da resposta ao impulso do canal foi caracterizada. 
Mostrou-se que esta tarefa consiste num problema NP-hard, normalmente inviável devido ao tamanho típico da cardinalidade do suporte da resposta ao impulso do canal, justificando assim os métodos sub-ótimos de estimação apresentados a seguir.

O primeiro método sub-ótimo de estimação apresentada foi o método de Encolhimentos Sucessivos. Foi mostrado que este método fundamenta-se no Teorema 3.1, em que se demonstra a monotonicidade da sequência de custos LS associadas às estimativas LS com suporte reduzido, à medida em que o suporte estimado sofre encolhimentos sucessivos. Para implementação deste método, foi proposto um novo algoritmo de decimação iterativa, de baixo custo computacional. Foram também apresentadas diversas simulações comparando o algoritmo proposto com os algoritmos de minimização do custo LS Fullsupport e custo LS Oráculo (com suporte conhecido). Por último, foi feita uma análise do algoritmo proposto em termos de custo computacional.

O segundo método sub-ótimo de estimação apresentado foi o método de Expansões Sucessivas, que foi inspirado no algoritmo de Matching Pursuit. Foram também apresentadas diversas simulações comparando o algoritmo proposto com os algoritmos de minimização do custo LS Full-support e custo LS Oráculo (com suporte conhecido). Por último, foi feita uma análise do algoritmo proposto em termos de custo computacional.

O terceiro método sub-ótimo de estimação apresentado foi o método de Minimização da Norma $l_{1}$, Precedendo a apresentação deste método, foram apresentados os algoritmos correlatos Basis Pursuit e LASSO. Foram também apresentadas diversas simulações comparando o algoritmo proposto com os algoritmos de minimização do custo LS Full-support e custo LS Oráculo (com suporte conhecido). Por último, foi feita uma análise do algoritmo proposto em termos de custo computacional.

O quarto método sub-ótimo de estimação apresentado foi o método de Regularização Automática do Custo LS. Inicialmente, os conceitos básicos de regularização foram apresentados. Em seguida, foi proposta uma modificação na função-custo LS com regularização. A partir dessa nova função-custo, foi proposto um critério para determinação do valor ótimo do fator de regularização, baseado no fato de que a variação do fator de regularização descreve uma curva de tradeoff entre o custo LS e a norma $l_{1}$ da estimativa produzida. A partir daí, foi proposto um mecanismo de ajuste automático do fator de regularização. Finalmente, foi proposto um novo algoritmo iterativo de regularização, com ajuste automático do parâmetro de regularização. Foram também apresentadas diversas simulações comparando o algoritmo proposto com os al- 
goritmos de minimização do custo LS Full-support e custo LS Oráculo (com suporte conhecido). Por último, foi feita uma análise do algoritmo proposto em termos de custo computacional.

No capítulo seguinte, os quatro métodos de estimação de sistemas esparsos originalmente propostos neste capítulo são comparados entre si e com o algoritmo $l_{1}$-RLS (20), em termos de desempenho e custo computacional, a partir do resultado de simulações. Esses cinco algoritmos são também comparados com os algoritmos LS full-support e LS Oráculo. As simulações apresentadas no próximo capítulo dividem-se em duas categorias: estimação de sistemas MESS e estimação de canais UWB esparsos, conforme o modelo descrito no documento "IEEE 805.15.4a channel model - final report" (27). 


\section{5}

\section{Avaliação de desempenho}

\section{1}

\section{Introdução}

Este capítulo tem como finalidade realizar uma avaliação de desempenho dos métodos de estimação de sistemas esparsos apresentados no capítulo anterior. Na seção a seguir, as versões despolarizadas dos quatro métodos de estimação de sistemas esparsos propostos nesta Tese são comparadas entre si e com os algoritmos LS Full-support, LS Oráculo e $l_{1}$-RLS, em termos de desempenho e de custo computacional, a partir dos resultados obtidos em simulações em que eles são empregados na estimação de sistemas modelados como MESS (o modelo de sistemas MESS é descrito no Anexo B). Na seção subsequente, estes métodos são avaliados em termos da taxa de erros de bits versus a razãosinal ruído, a partir dos resultados obtidos em simulações de um sistema de comunicações IR-UWB, conforme o modelo descrito no Anexo A, em que estes métodos são empregados na estimação de canais que seguem o modelo IEEE 802.15.4a (27). A seção seguinte destina-se à avaliação de custo computacional dos métodos apresentados. O capítulo termina com uma seção destinada a considerações finais.

\section{2}

\section{Avaliação de desempenho dos métodos quando empregados na estimação de sistemas MESS}

Nesta seção, as versões despolarizadas dos quatro métodos de estimação de sistemas esparsos originalmente concebidos nesta Tese são comparadas entre si, em termos de desempenho na estimação de sistemas MESS.

As Figuras 5.1 a 5.6 mostram o desempenho dos métodos de Encolhimentos Sucessivos, Expansões Sucessivas despolarizado, Minimização da Norma $l_{1}$ despolarizado, Regularização Iterativa Automática do Custo LS, LS Fullsupport e LS Oráculo, para $Q=1000$ realizações de um sistema $\operatorname{MESS}(100,10)$ e SINR variando de $5 \mathrm{~dB}$ até $30 \mathrm{~dB}$, com $N=16$ e $\lambda=1,05$. As Figuras 5.7 a 5.12 mostram o desempenho dos mesmo métodos, para sistema MESS(100,20), mantendo-se inalterados os outros parâmetros de simulação. 


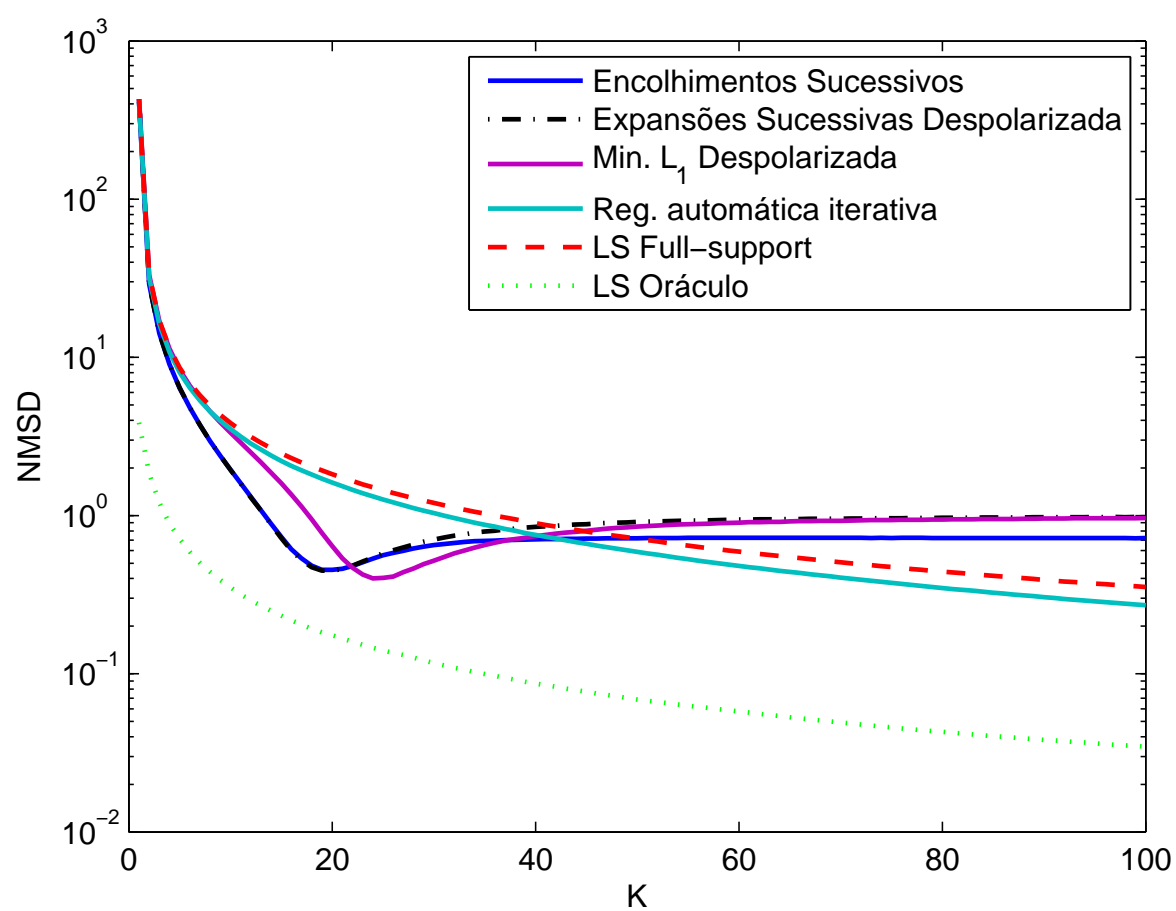

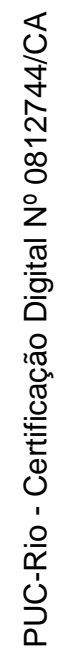

Figura 5.1: Comparação de desempenho: $\operatorname{MESS}(100,10), \operatorname{SINR}=5 \mathrm{~dB}$, $\lambda=1,05, N=16$ e $Q=1000$

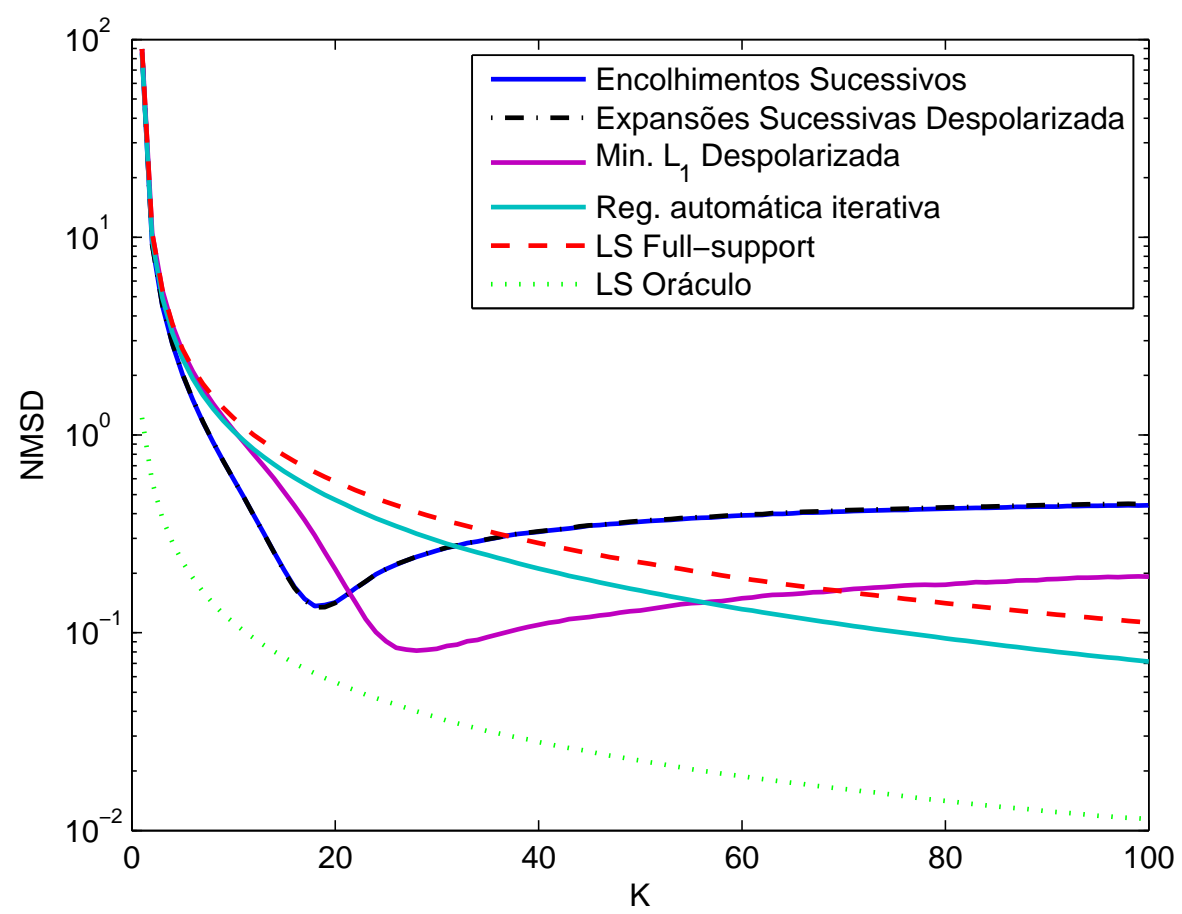

Figura 5.2: Comparação de desempenho: $\operatorname{MESS}(100,10), \operatorname{SINR}=10 \mathrm{~dB}$, $\lambda=1,05, N=16$ e $Q=1000$ 


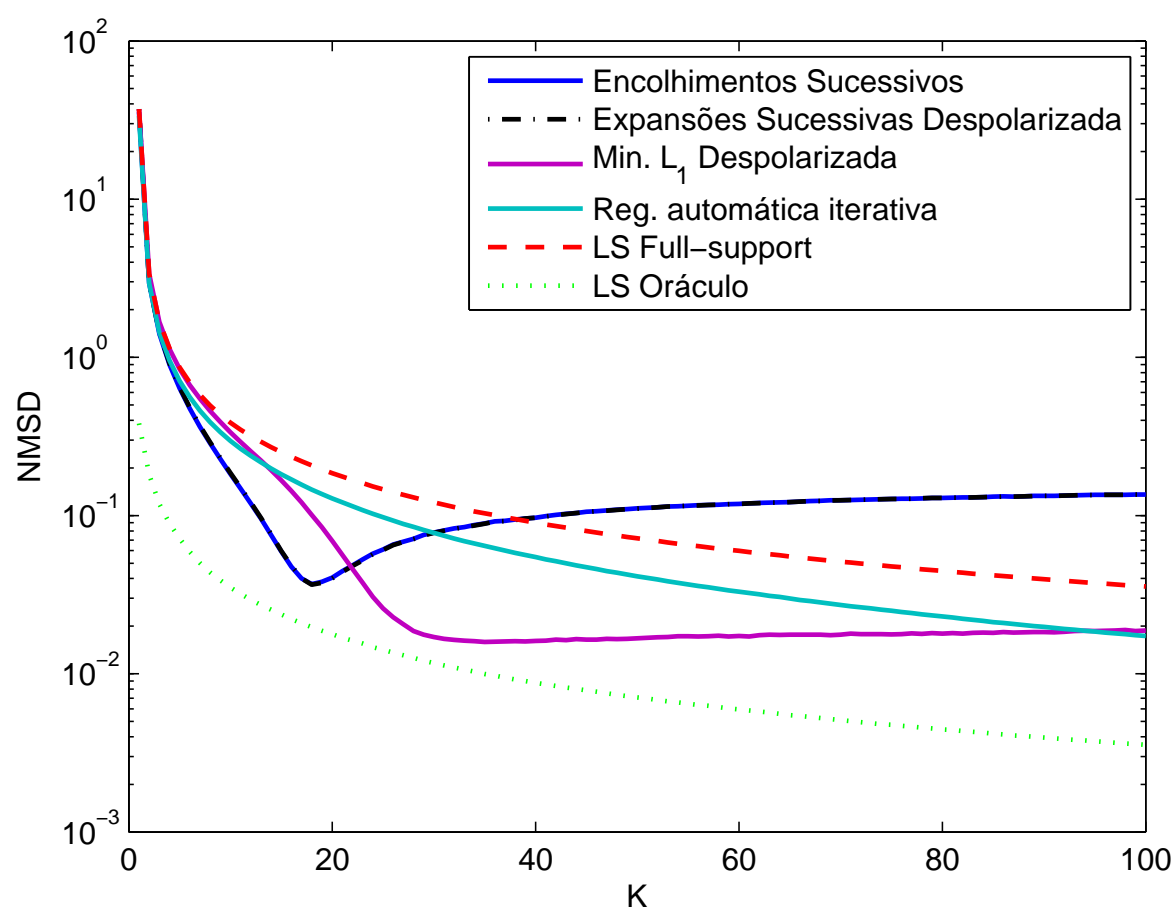

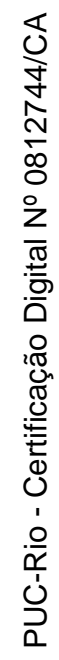

Figura 5.3: Comparação de desempenho: $\operatorname{MESS}(100,10), \mathrm{SINR}=15 \mathrm{~dB}$, $\lambda=1,05, N=16$ e $Q=1000$

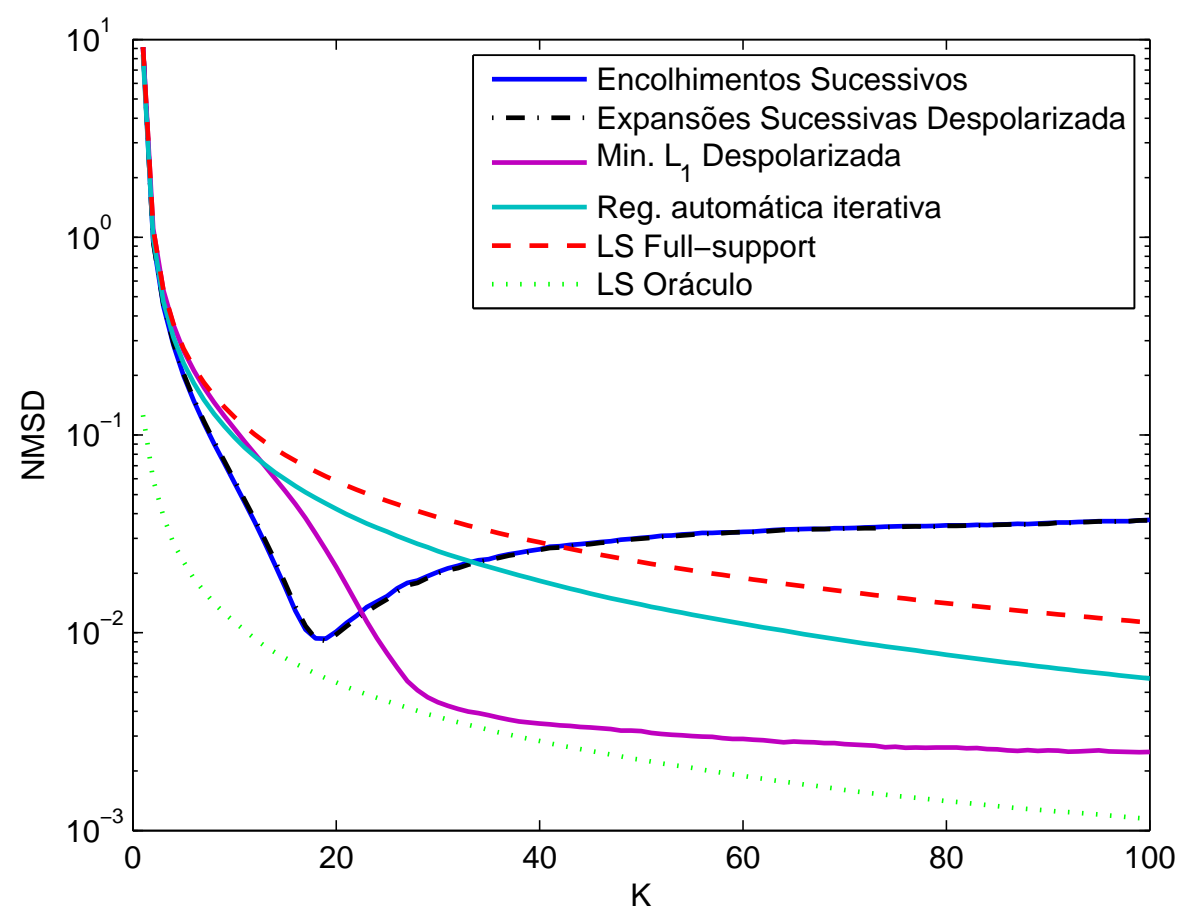

Figura 5.4: Comparação de desempenho: $\operatorname{MESS}(100,10), \operatorname{SINR}=20 \mathrm{~dB}$, $\lambda=1,05, N=16$ e $Q=1000$ 


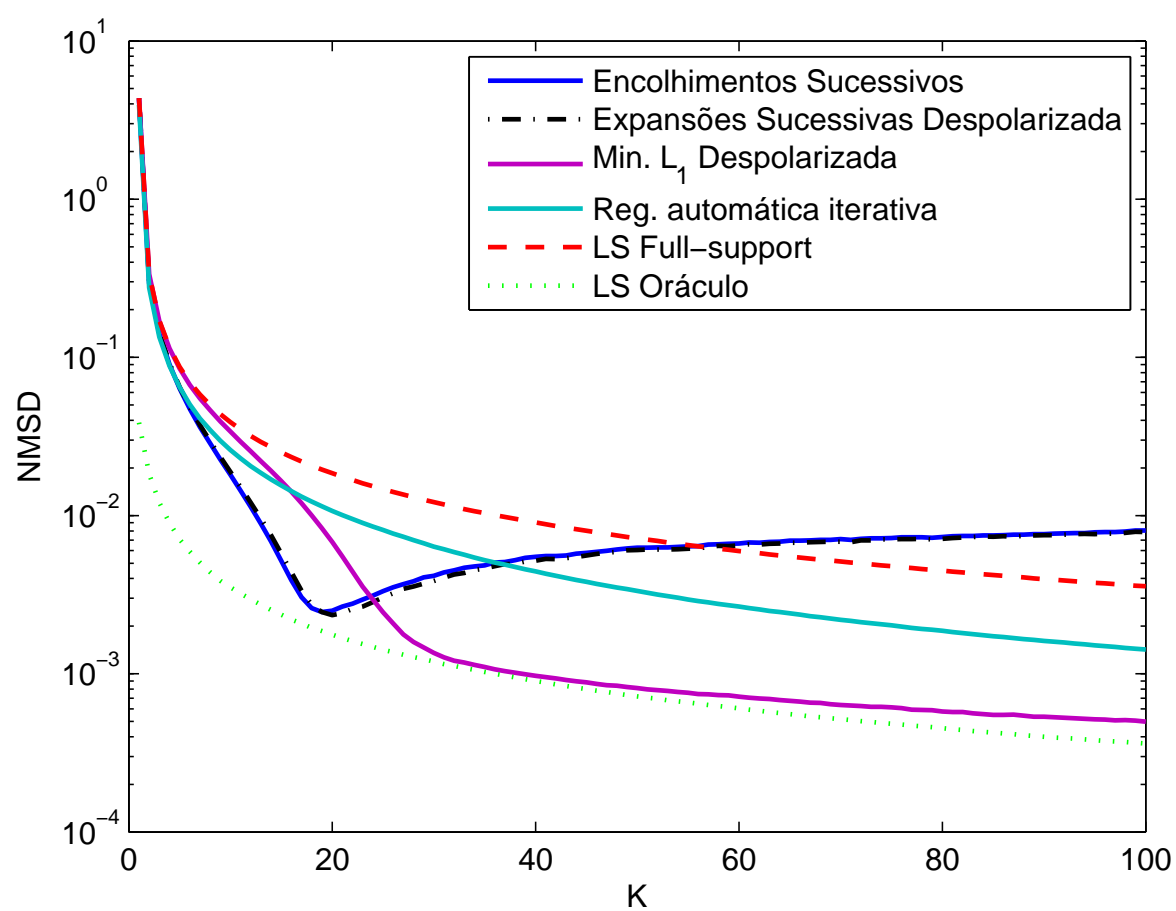

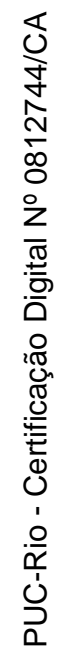

Figura 5.5: Comparação de desempenho: $\operatorname{MESS}(100,10), \operatorname{SINR}=25 \mathrm{~dB}$, $\lambda=1,05, N=16$ e $Q=1000$

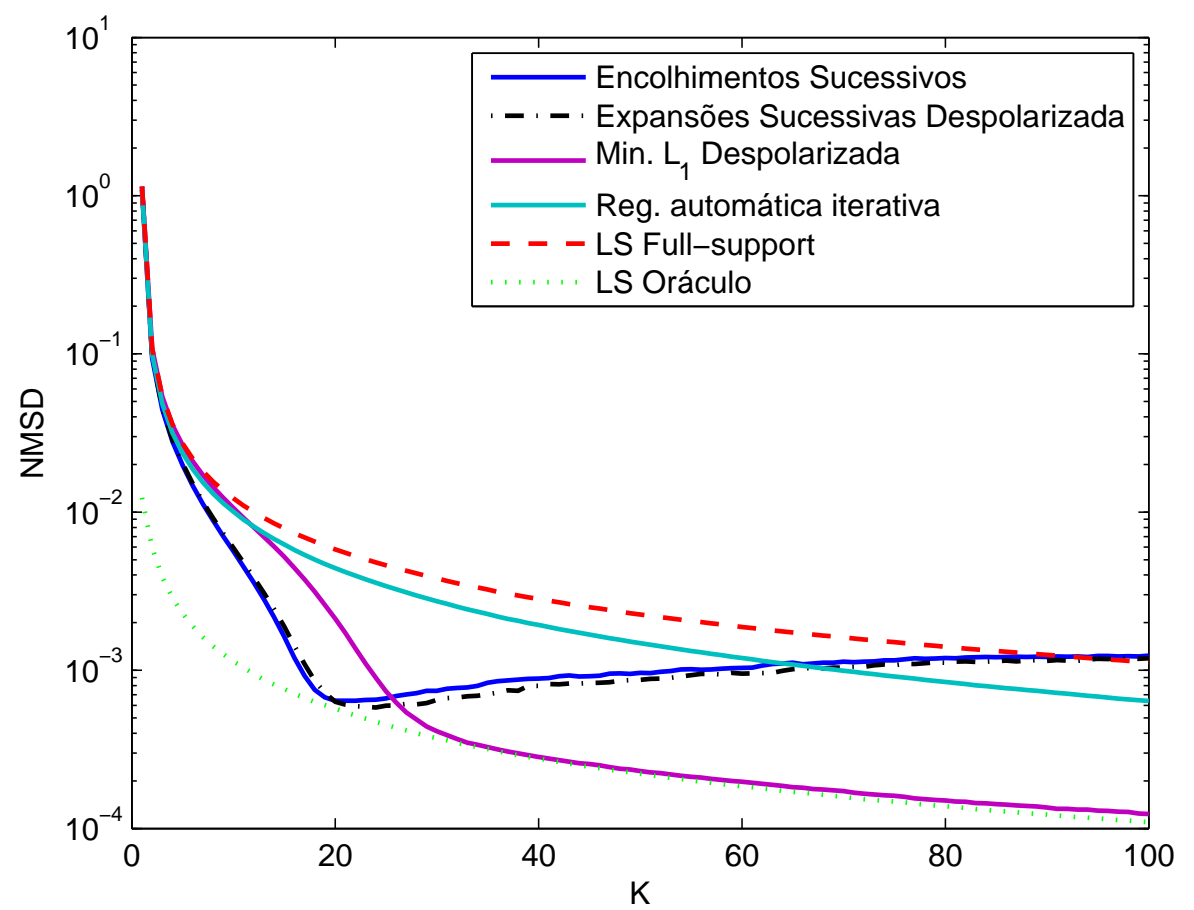

Figura 5.6: Comparação de desempenho: $\operatorname{MESS}(100,10), \operatorname{SINR}=30 \mathrm{~dB}$, $\lambda=1,05, N=16$ e $Q=1000$ 


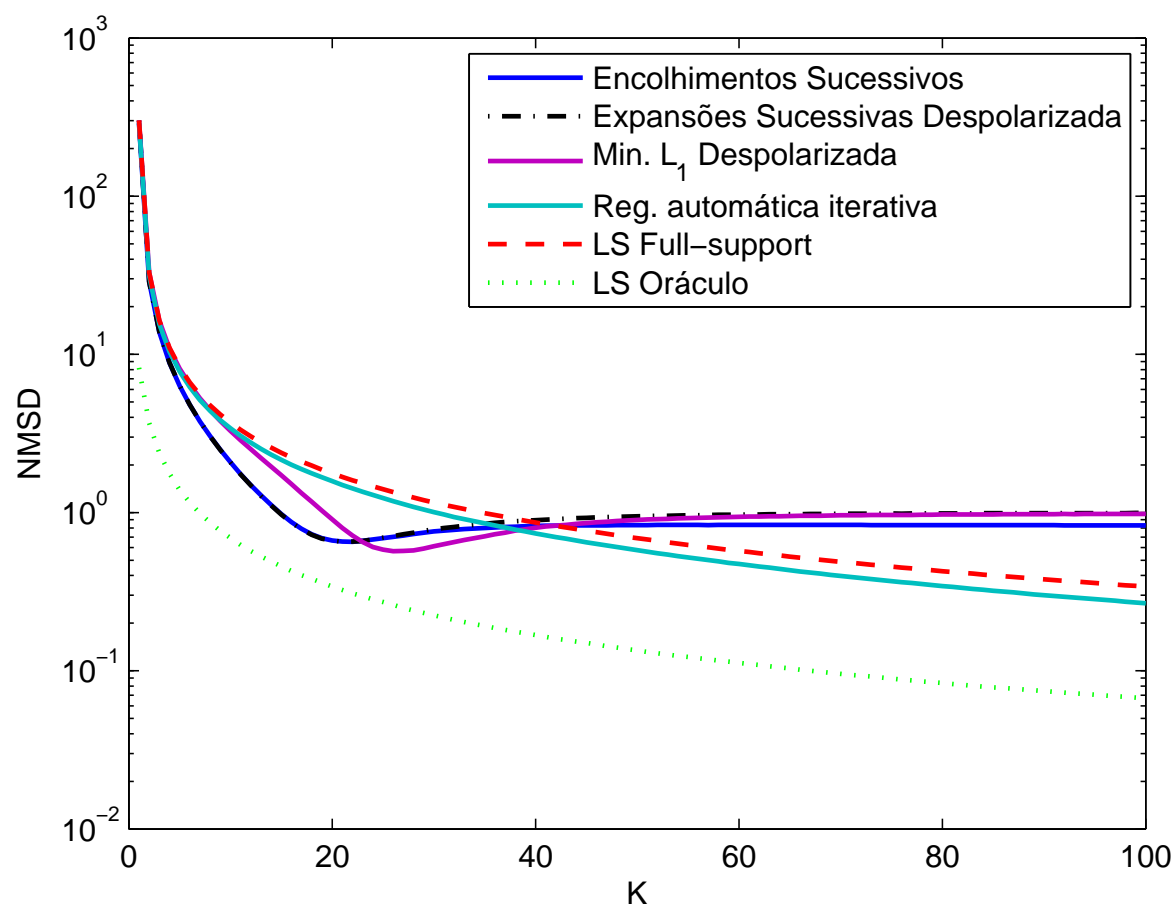

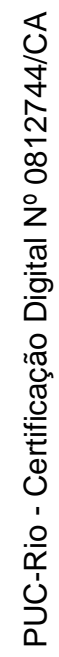

Figura 5.7: Comparação de desempenho: $\operatorname{MESS}(100,20), \operatorname{SINR}=5 \mathrm{~dB}$, $\lambda=1,05, N=16$ e $Q=1000$

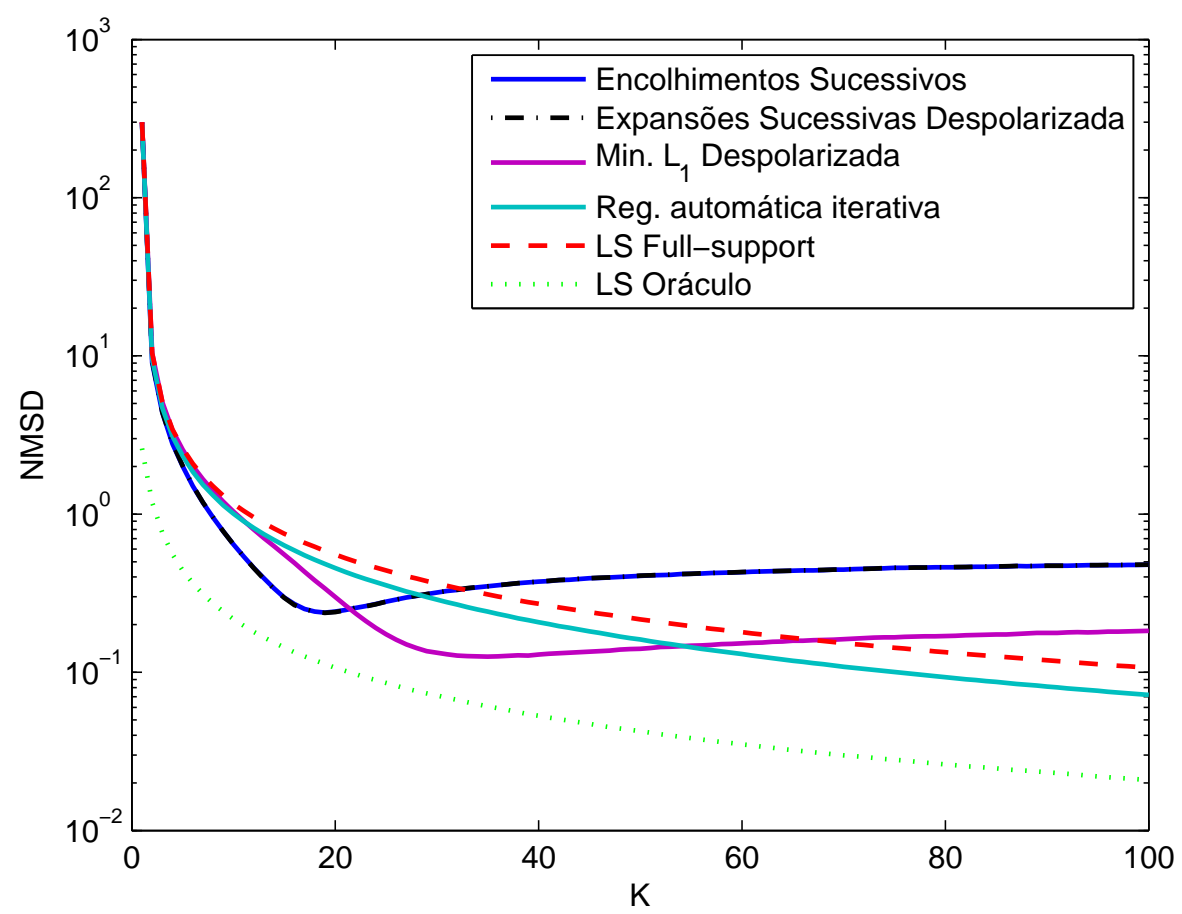

Figura 5.8: Comparação de desempenho: $\operatorname{MESS}(100,20), \operatorname{SINR}=10 \mathrm{~dB}$, $\lambda=1,05, N=16$ e $Q=1000$ 


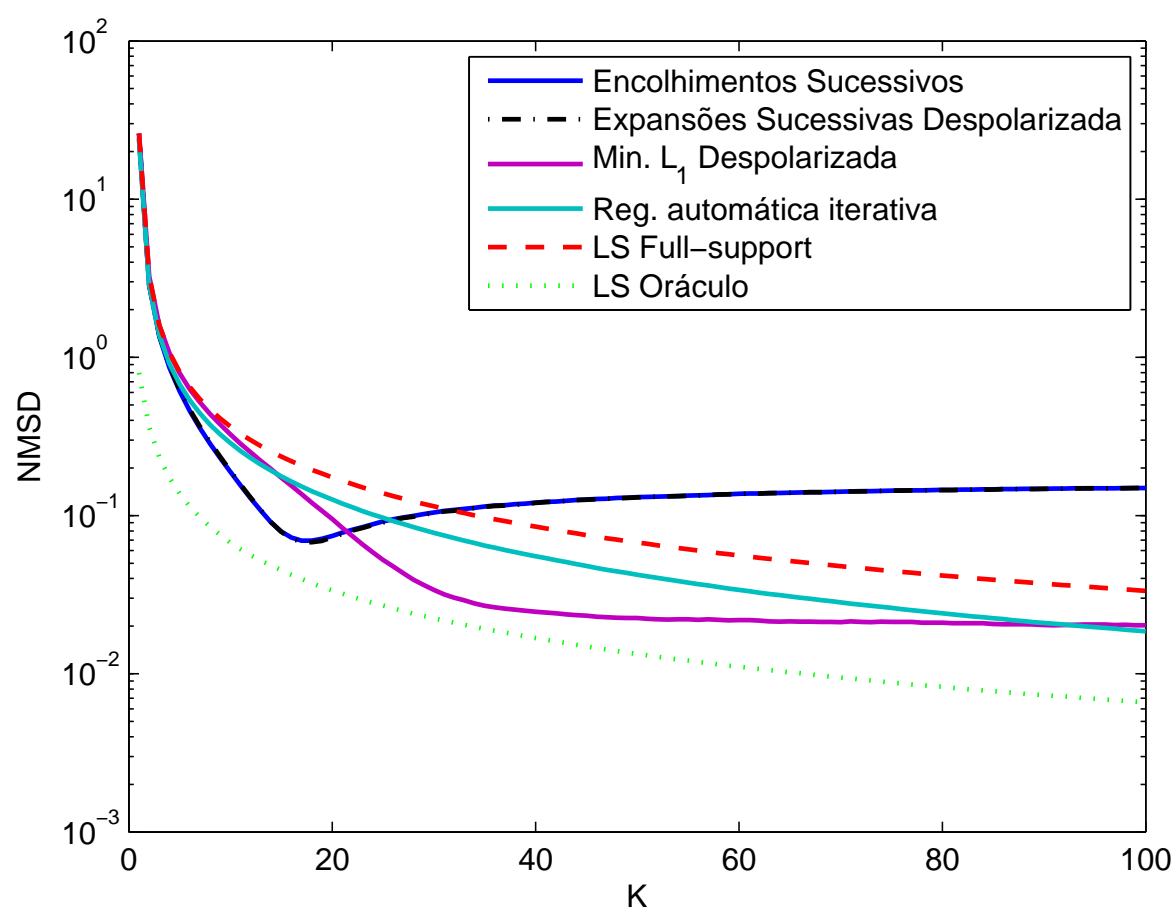

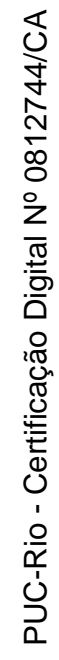

Figura 5.9: Comparação de desempenho: $\operatorname{MESS}(100,20), \quad \mathrm{SINR}=15 \mathrm{~dB}$, $\lambda=1,05, N=16$ e $Q=1000$

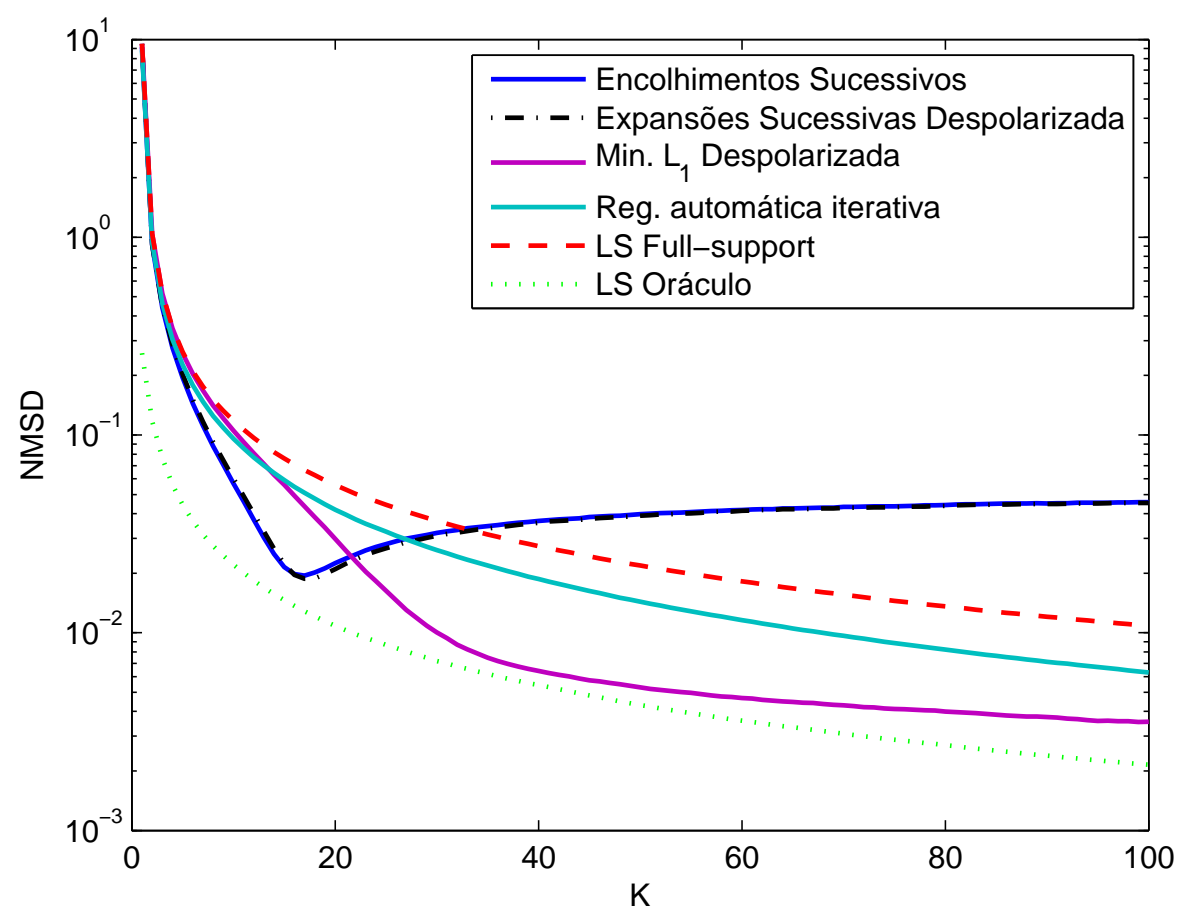

Figura 5.10: Comparação de desempenho: $\operatorname{MESS}(100,20), \operatorname{SINR}=20 \mathrm{~dB}$, $\lambda=1,05, N=16$ e $Q=1000$ 


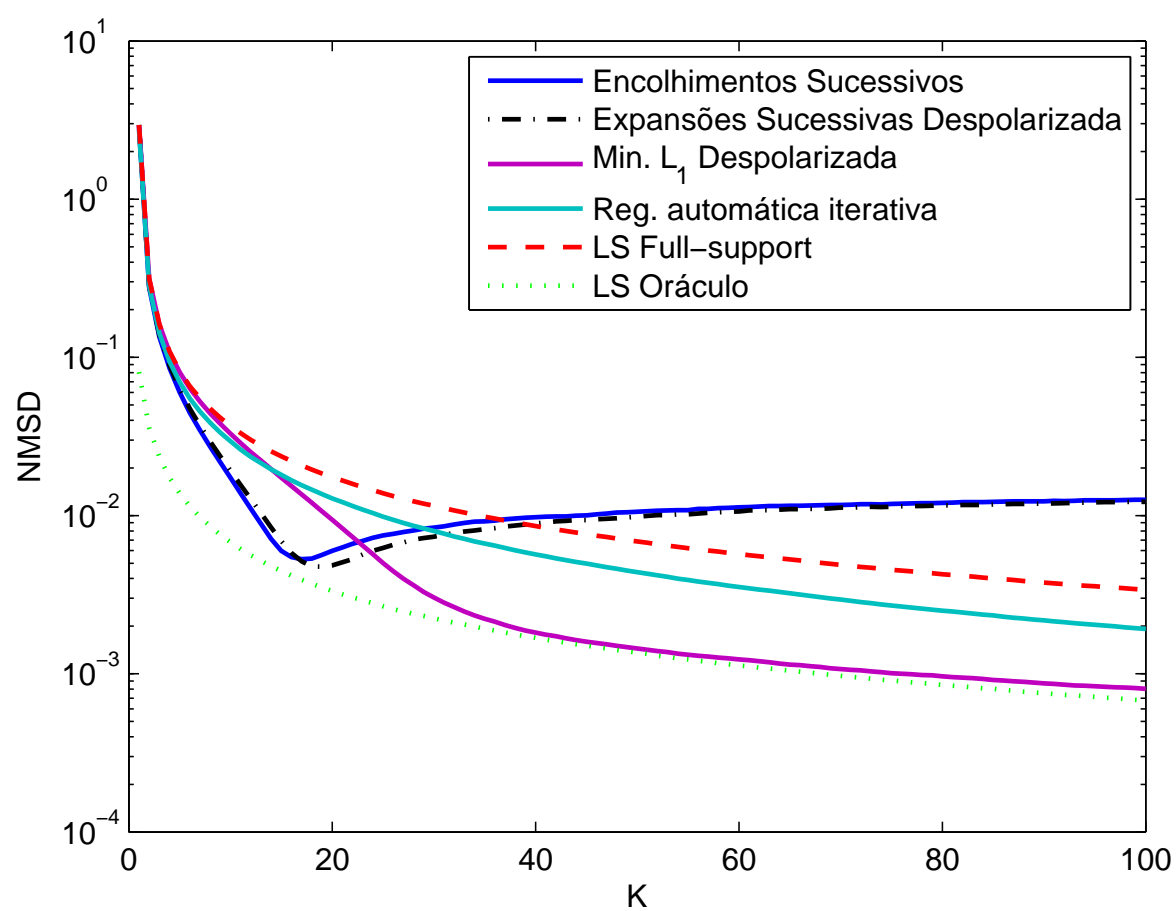

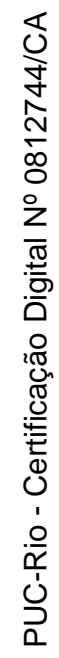

Figura 5.11: Comparação de desempenho: $\operatorname{MESS}(100,20)$, SINR $=25 \mathrm{~dB}$, $\lambda=1,05, N=16$ e $Q=1000$

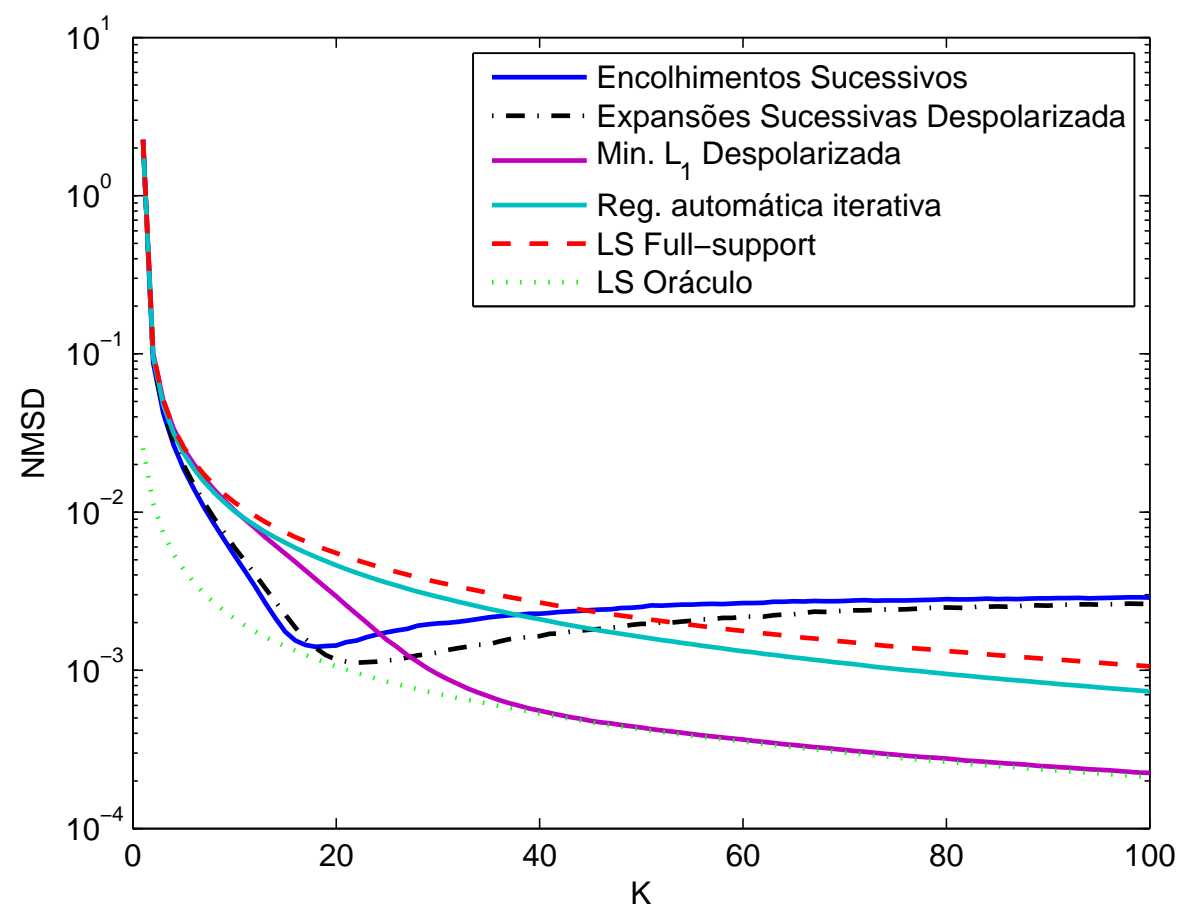

Figura 5.12: Comparação de desempenho: $\operatorname{MESS}(100,20)$, SINR $=30 \mathrm{~dB}$, $\lambda=1,05, N=16$ e $Q=1000$ 
Para que a comparação de desempenho não ficasse restrita aos métodos originalmente apresentados nesta Tese, o algoritmo $l_{1}$-RLS, apresentado em (20), foi eleito com essa finalidade, por se tratar de um algoritmo baseado na técnica de estimação LS, assim como os demais. A Figura 5.13 mostra o desempenho dos métodos de Encolhimentos Sucessivos, Expansões Sucessivas despolarizado, Minimização da Norma $l_{1}$ despolarizado, Regularização Iterativa Automática do Custo LS, LS Full-support, LS Oráculo e $l_{1}$-RLS, nas mesmas condições de de avaliação apresentadas em (20), que são: $Q=100$ realizações de um sistema $\operatorname{MESS}(64,8), \mathrm{SINR}=20 \mathrm{~dB} \operatorname{com} N=1000, M=1$ e $\lambda=1,2$. O algoritmo $l_{1}$-RLS foi testado com os mesmos valores de parâmetros adotados em (20): $\mathrm{ff}=0,995$ (fator de esquecimento) e fr=1,5 (fator de regularização). Verifica-se que o patamar de NMSD estacionário do algoritmo $l_{1}$-RLS é maior que dos demais algoritmos, devido ao fato de que $(\mathrm{ff}<1)$, pois o efeito de regularização só ocorre quando $(\mathrm{ff}<1)$, o que consiste em uma limitação do algoritmo $l_{1}$-RLS.

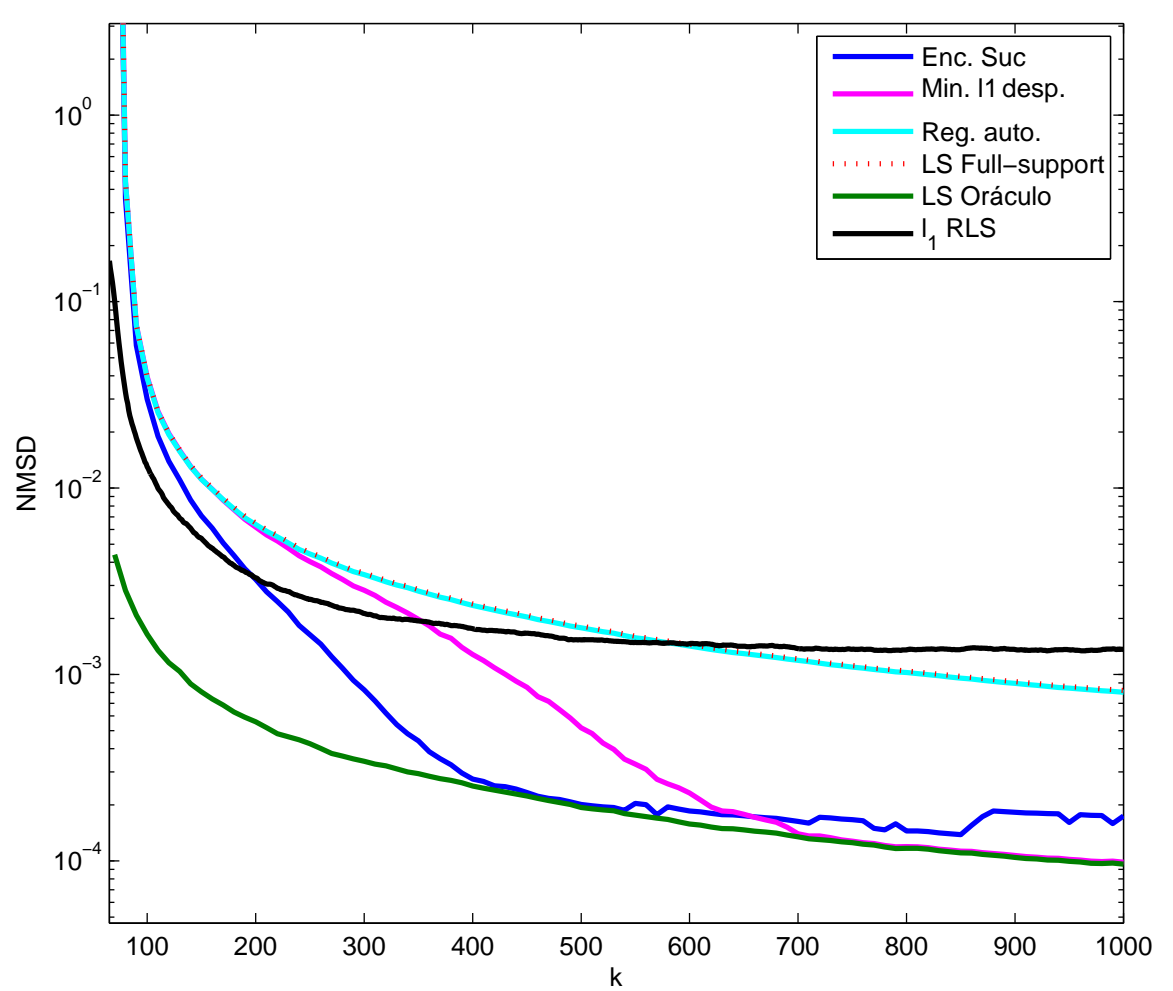

Figura 5.13: Comparação de desempenho entre os métodos de Encolhimentos Sucessivos, Expansões Sucessivas despolarizada, Minimização da norma $l_{1}$ despolarizada, Ajuste automático do fator de regularização do custo LS, LS Full-support, LS Oráculo e $l_{1}$-RLS, com: $\operatorname{MESS}(64,8), \operatorname{SINR}=20 \mathrm{~dB}, \lambda=1,2$, $N=1000, M=1$ e $Q=1000$ 


\section{3}

\section{Avaliação de desempenho dos métodos quando empregados na estimação de canais IEEE 802.15.4a}

Nesta seção, os quatro métodos de estimação de sistemas esparsos originalmente apresentados nesta Tese e os algoritmos LS Full-support, LS Oráculo e $l_{1}$-RLS são comparados entre si, em termos de taxa de erros de bits versus razão sinal-ruído, quando empregados na estimação de canais em sistemas IRUWB, conforme modelo de sinais apresentado no Anexo A. Nas simulações, o canal equivalente discreto foi gerado a partir da amostragem, com intervalo de 2ns, da convolução entre o filtro de transmissão, o filtro de recepção (ambos do tipo raiz de cosseno levantado com fator de rolloff igual a 0,35 ) e o modelo IEEE 802.15.4a de canal para ambientes outdoor, descrito em (27).

As Figuras 5.14 a 5.29 mostram, de forma intercalada, a variação do fator de casamento (a definição do fator de casamento é apresentada no Anexo A) e da taxa de erros de bits (BER) em função do tamanho da sequência de treinamento, para valores de razão sinal-ruído variando de $6 \mathrm{~dB}$ a $20 \mathrm{~dB}$, mantendo-se os seguintes parâmetros dos algoritmos: $\mathrm{N}=32$ e $\lambda=1,01$. Em cada figura, foram geradas $Q=1000$ realizações de canal, e a taxa de erros de bits foi obtida de forma semi-analítica, a partir do fator de casamento entre cada uma dessas realizações e sua respectiva estimativa, conforme apresentado no Anexo A. Convém observar que, quanto menor o fator de casamento (que pode variar de -1 a 1), maior a taxa de erro de bits e, à medida em que o fator de casamento aproxima-se de 1 , mais a taxa de erro de bits aproxima-se do seu valor teórico para canais lineares e invariantes no tempo com ruído aditivo gaussiano branco. Constata-se que o fator de casamento varia de forma diferente para cada método de estimação avaliado, sendo que os métodos de Encolhimentos Sucessivos, Expansões Sucessivas e Minimização da norma $l_{1}$ alcançam o fator de casamento máximo com uma sequência de treinamento mais curta que o método de Regularização Automática do Custo LS.

A Figura 5.30 mostra o desempenho em termos da menor taxa de erros de bits alcançada por cada método versus a razão sinal-ruído, a partir da consolidação dos resultados mostrados nos gráficos antecessores.

\section{4}

\section{Avaliação do custo computacional}

A Figura 5.31 mostra o custo computacional dos métodos apresentados, medido através do tempo de processamento dos mesmos, nas simulações 


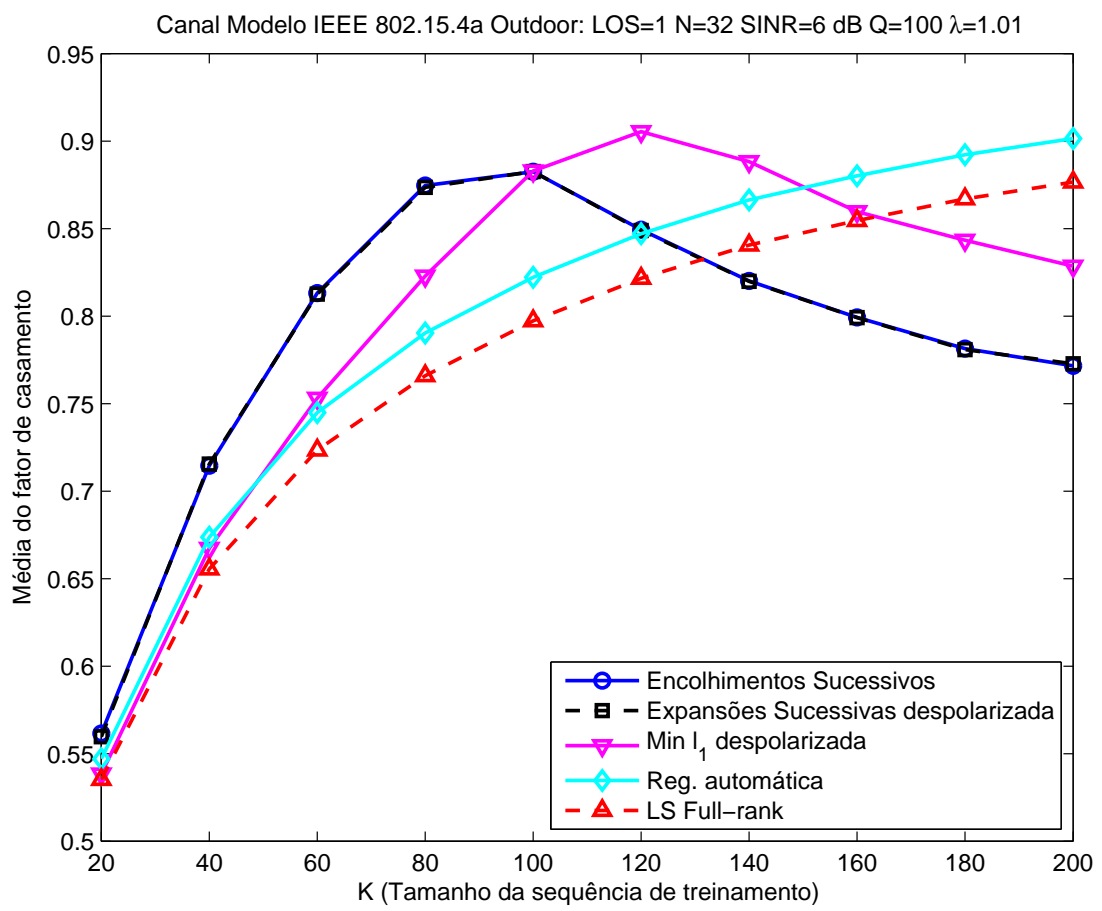

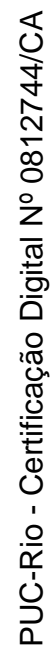

Figura 5.14: Fator de casamento na estimação do canal IEEE 802.15.4a (6dB)

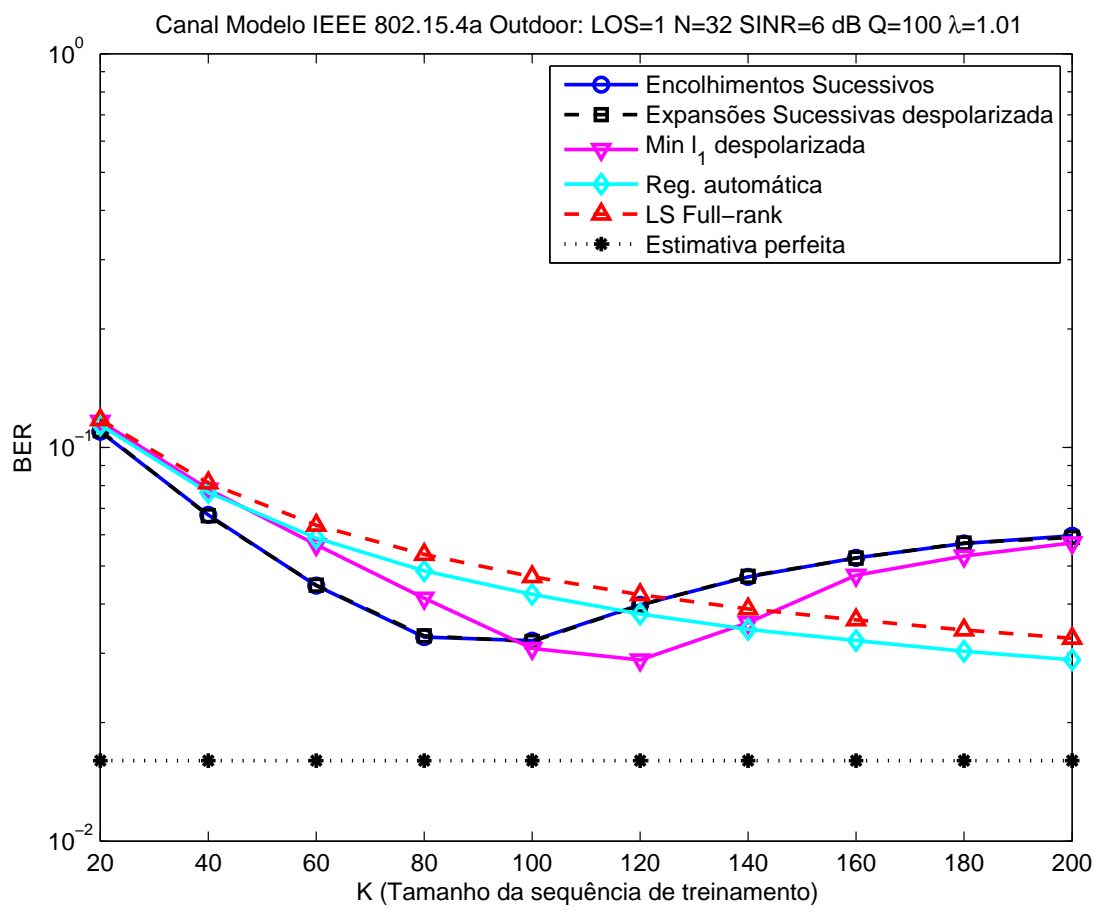

Figura 5.15: Taxa de erro de bits na estimação do canal IEEE 802.15.4a (6dB) 


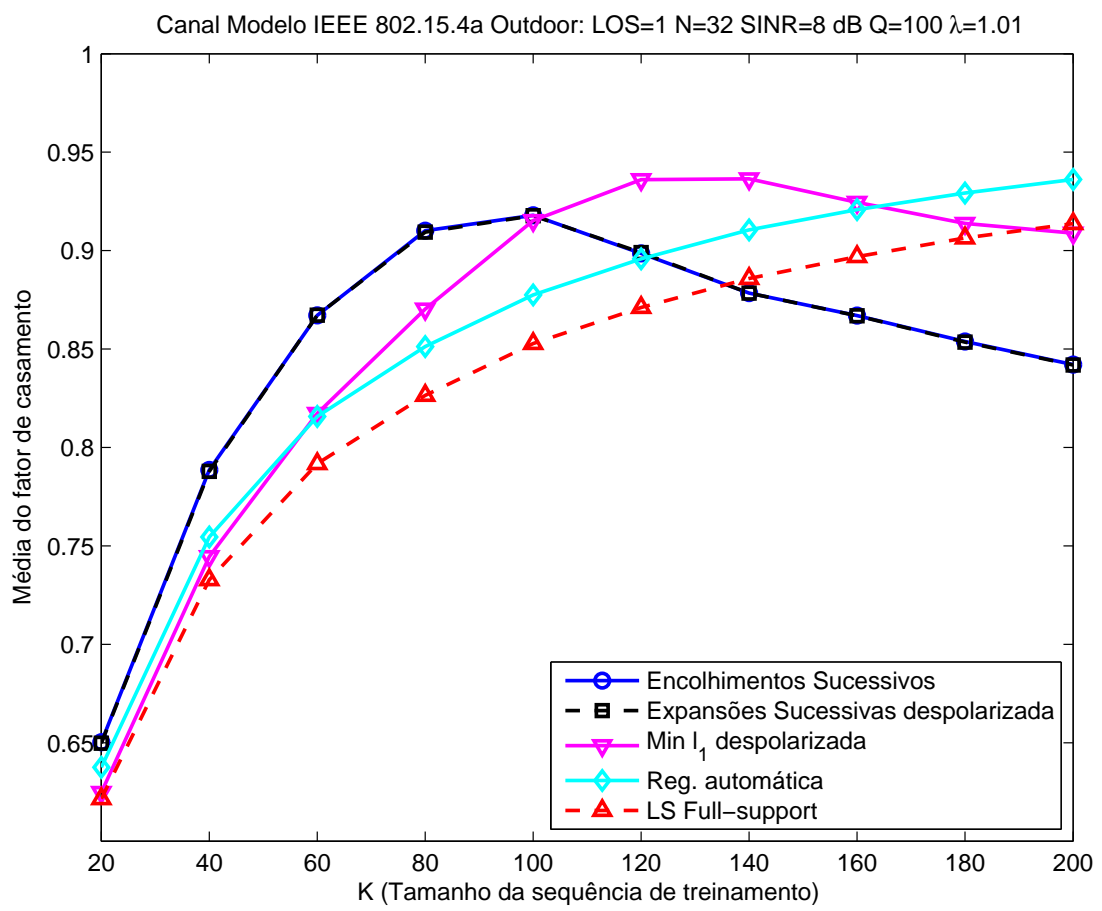

Figura 5.16: Fator de casamento na estimação do canal IEEE 802.15.4a (8dB)

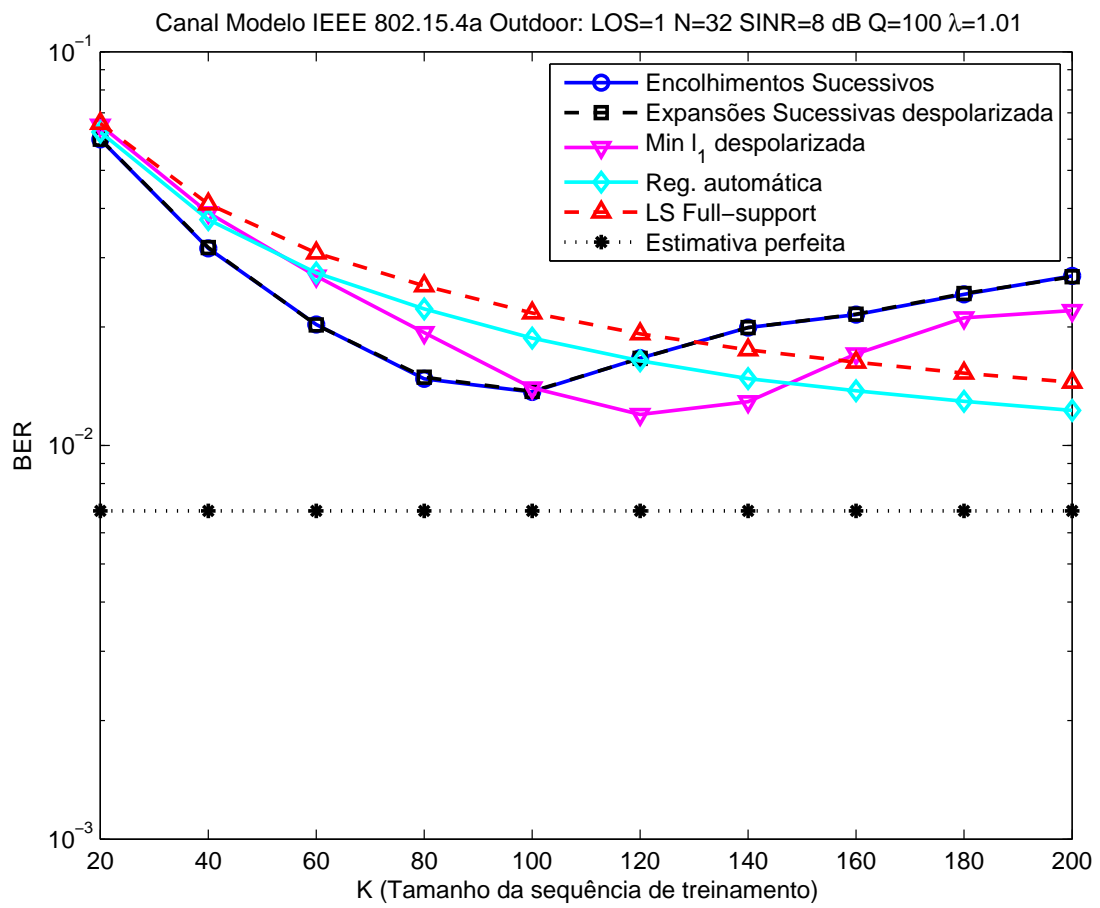

Figura 5.17: Taxa de erro de bits na estimação do canal IEEE 802.15.4a (8dB) 


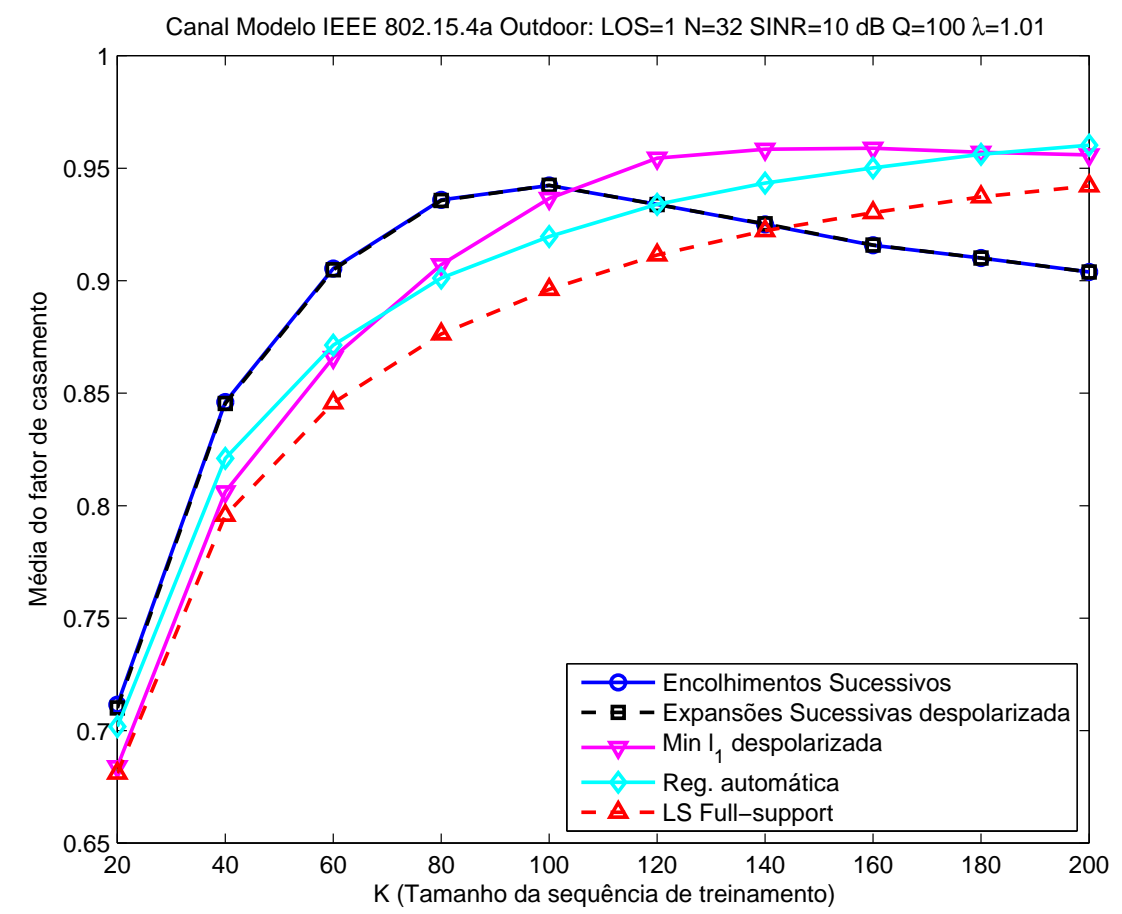

Figura 5.18: Fator de casamento na estimação do canal IEEE 802.15.4a (10dB)

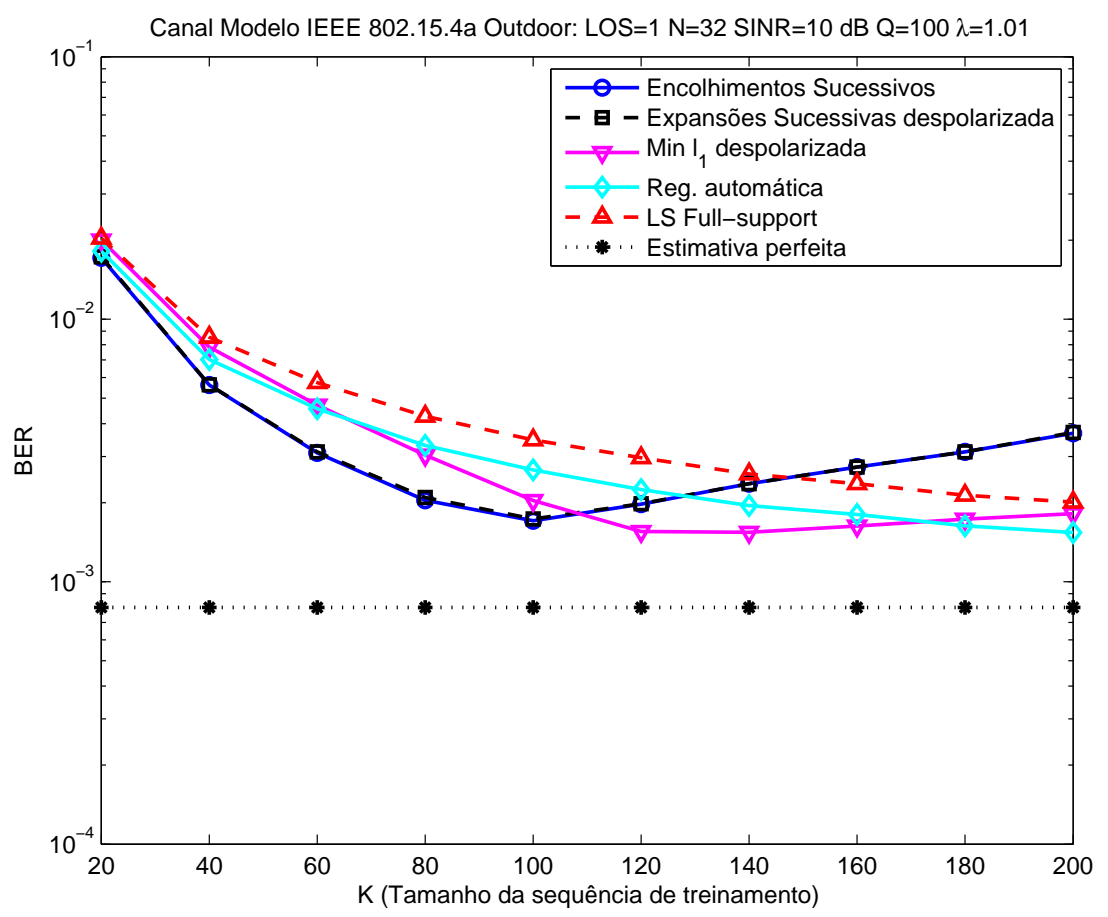

Figura 5.19: Taxa de erro de bits na estimação do canal IEEE 802.15.4a (10dB) 


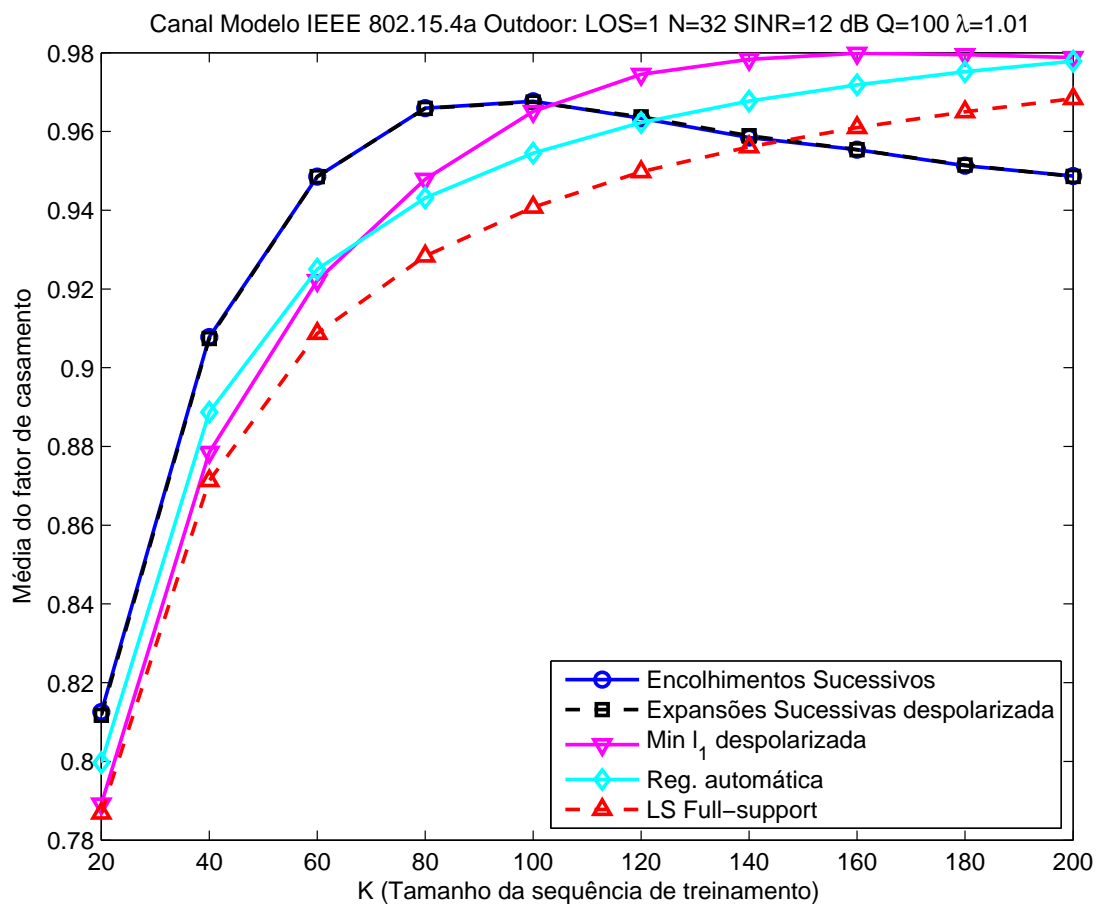

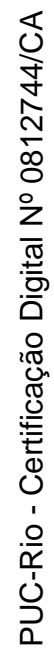

Figura 5.20: Fator de casamento na estimação do canal IEEE 802.15.4a (12dB)

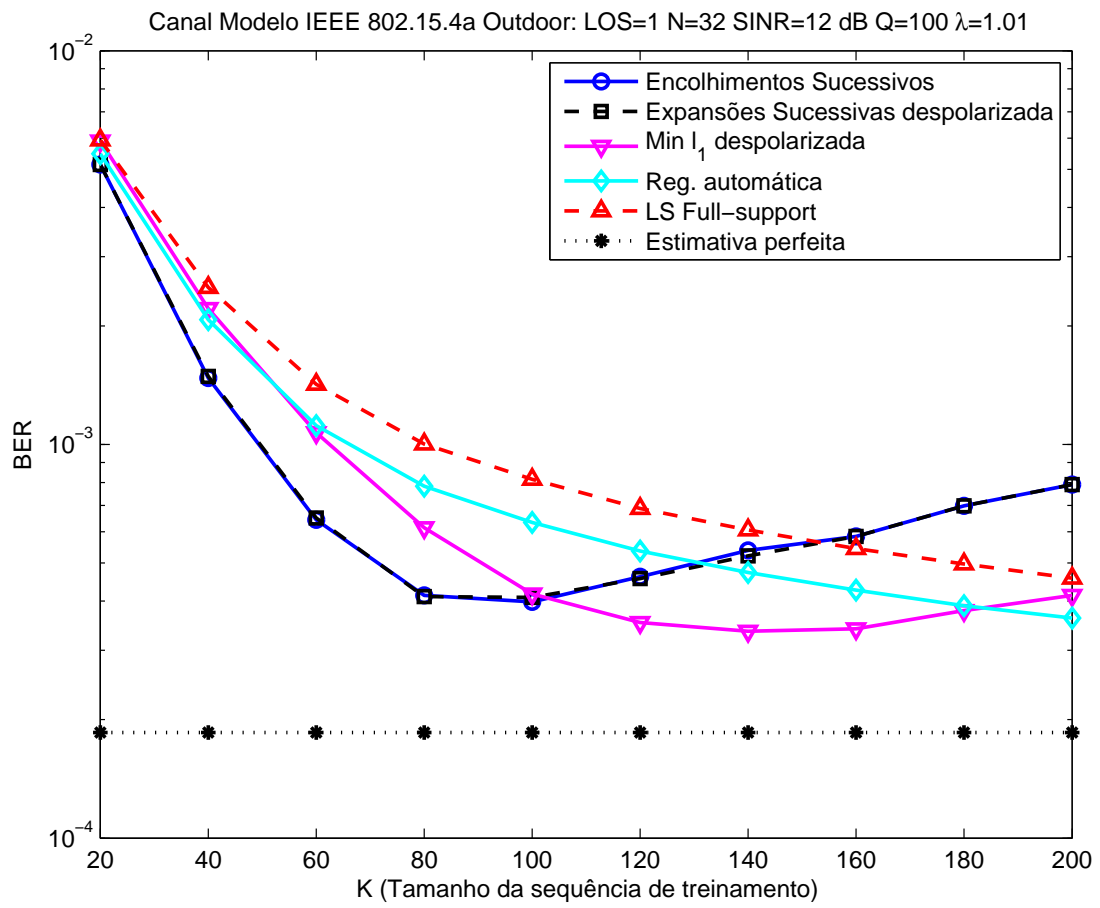

Figura 5.21: Taxa de erro de bits na estimação do canal IEEE 802.15.4a (12dB) 


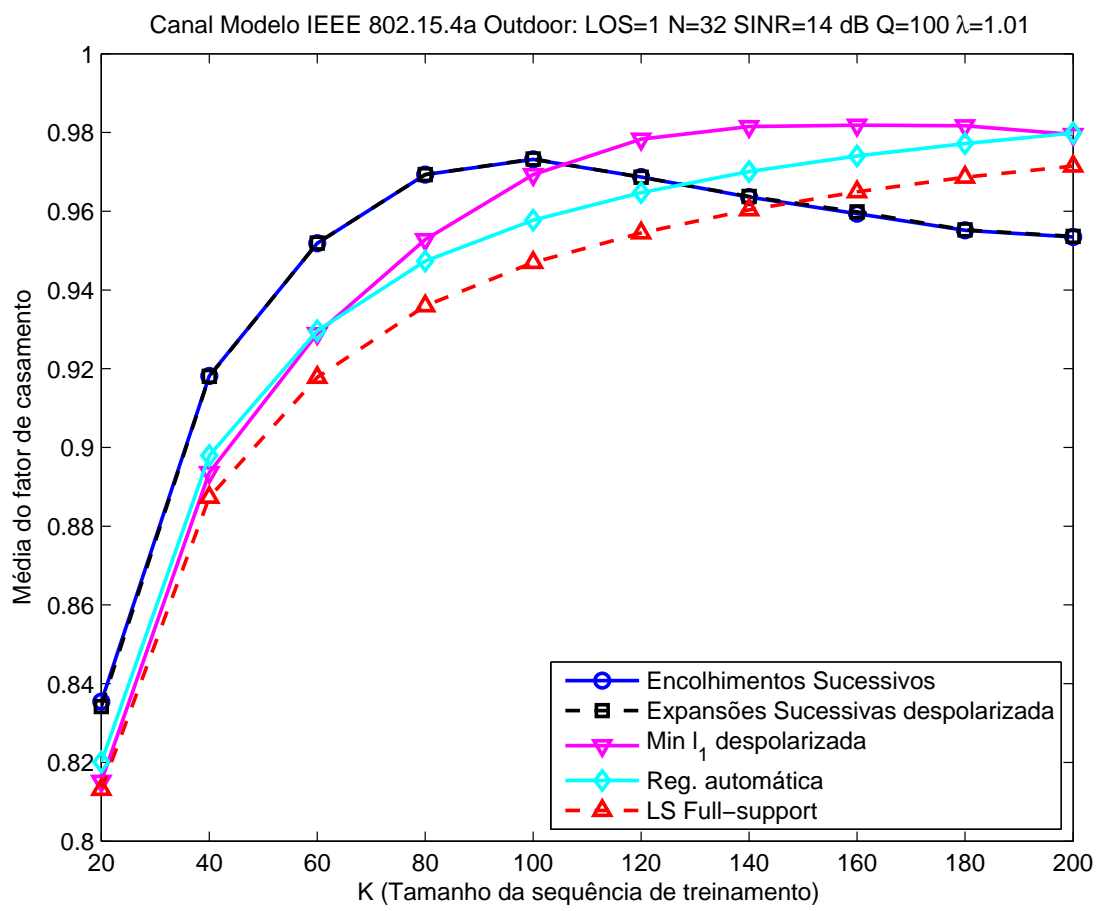

Figura 5.22: Fator de casamento na estimação do canal IEEE 802.15.4a (14dB)

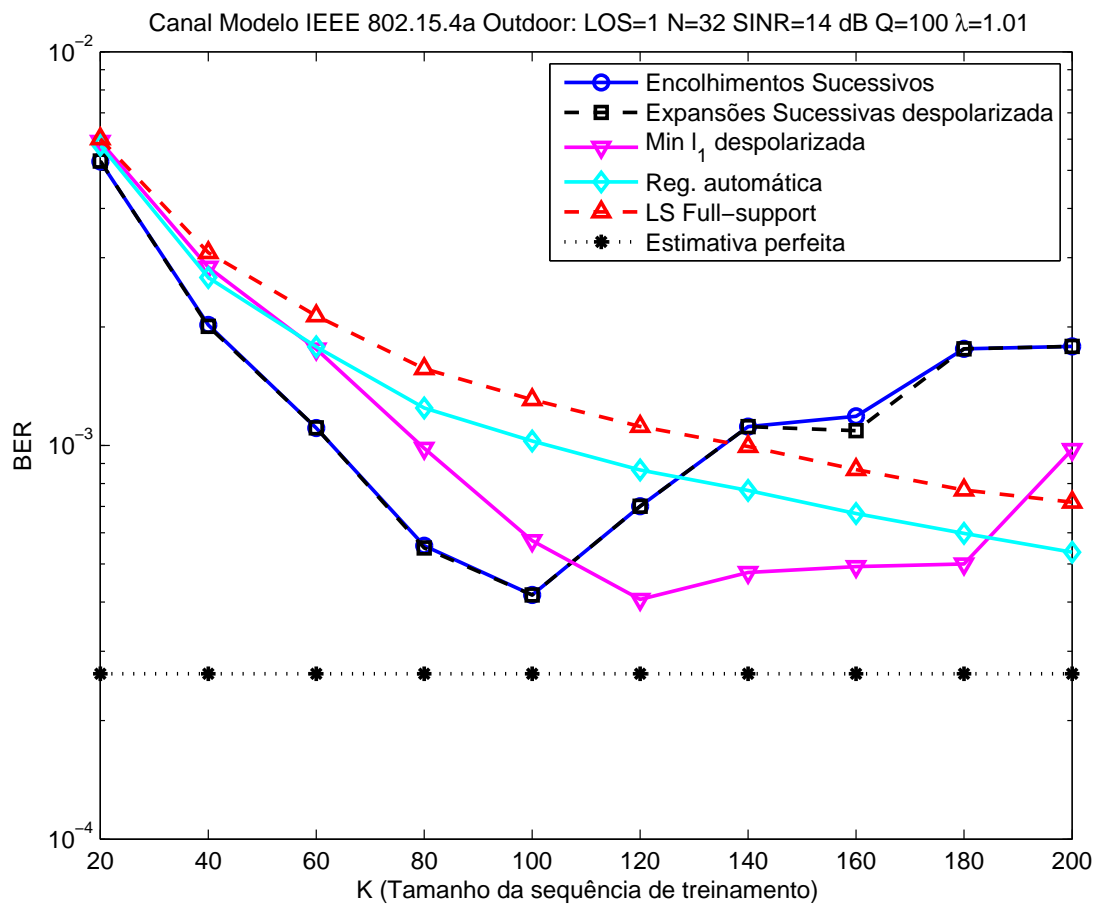

Figura 5.23: Taxa de erro de bits na estimação do canal IEEE 802.15.4a (14dB) 


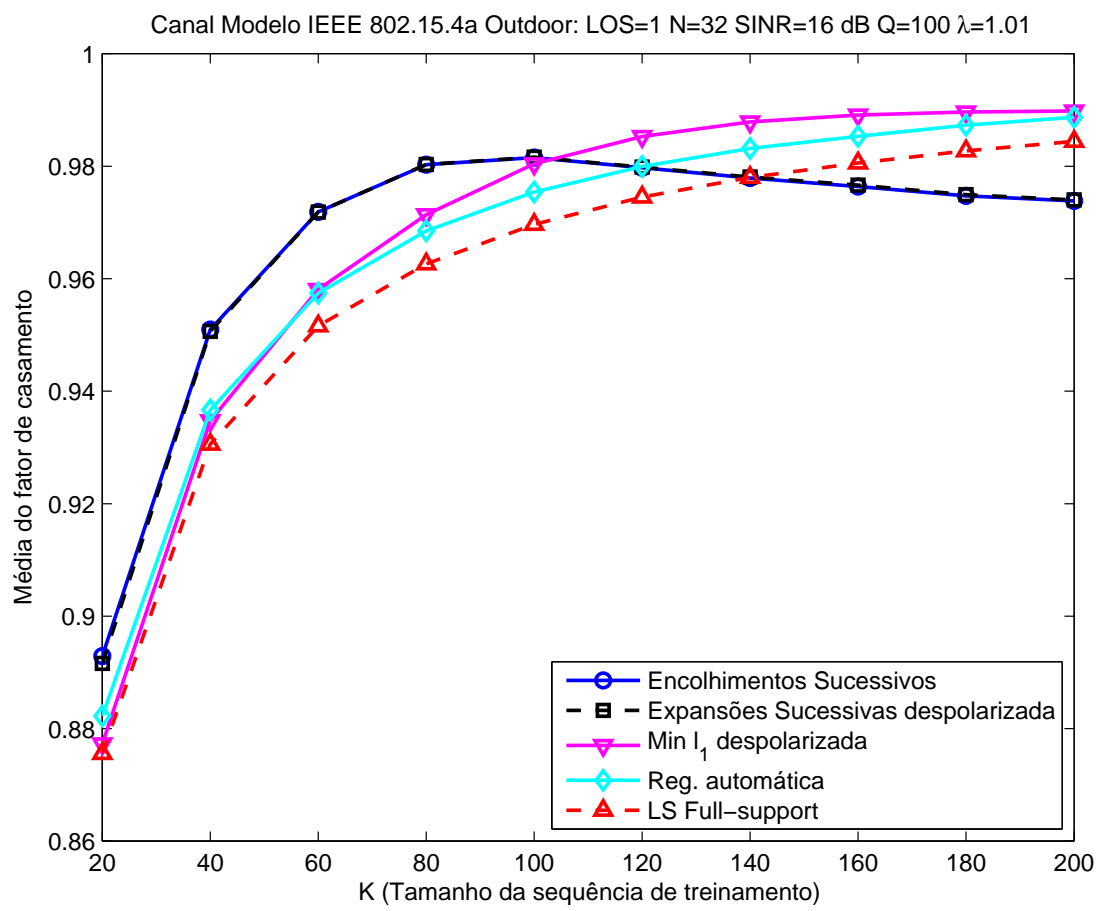

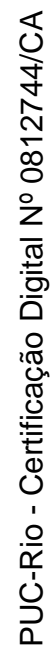

Figura 5.24: Fator de casamento na estimação do canal IEEE 802.15.4a (16dB)

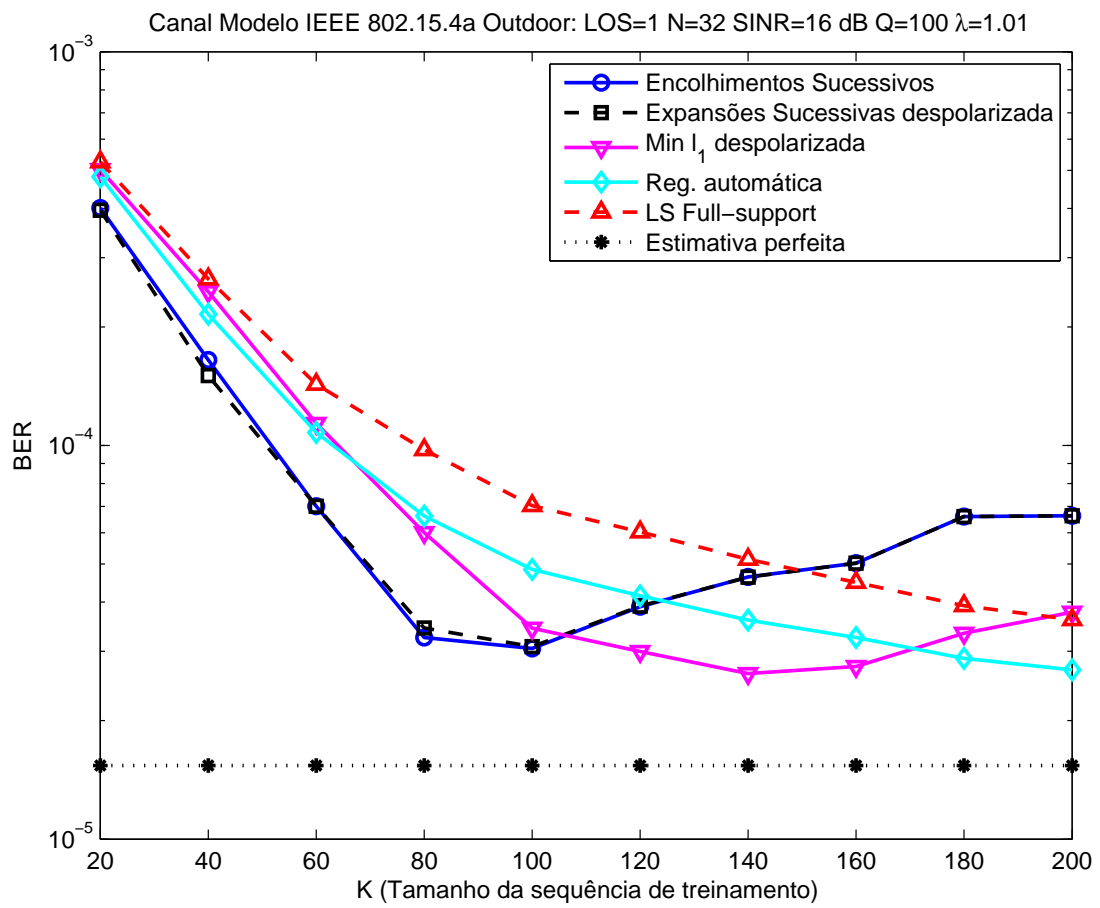

Figura 5.25: Taxa de erro de bits na estimação do canal IEEE 802.15.4a (16dB) 


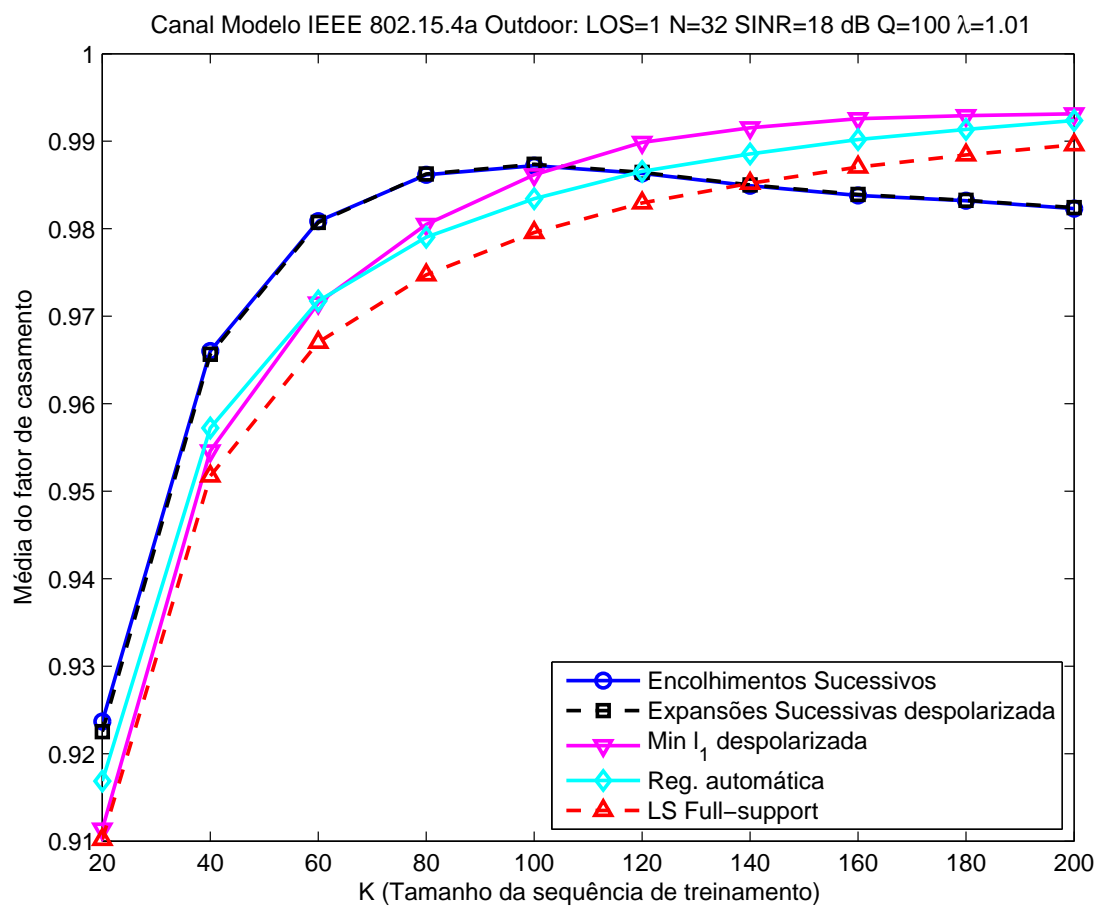

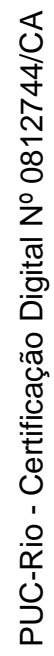

Figura 5.26: Fator de casamento na estimação do canal IEEE 802.15.4a (18dB)

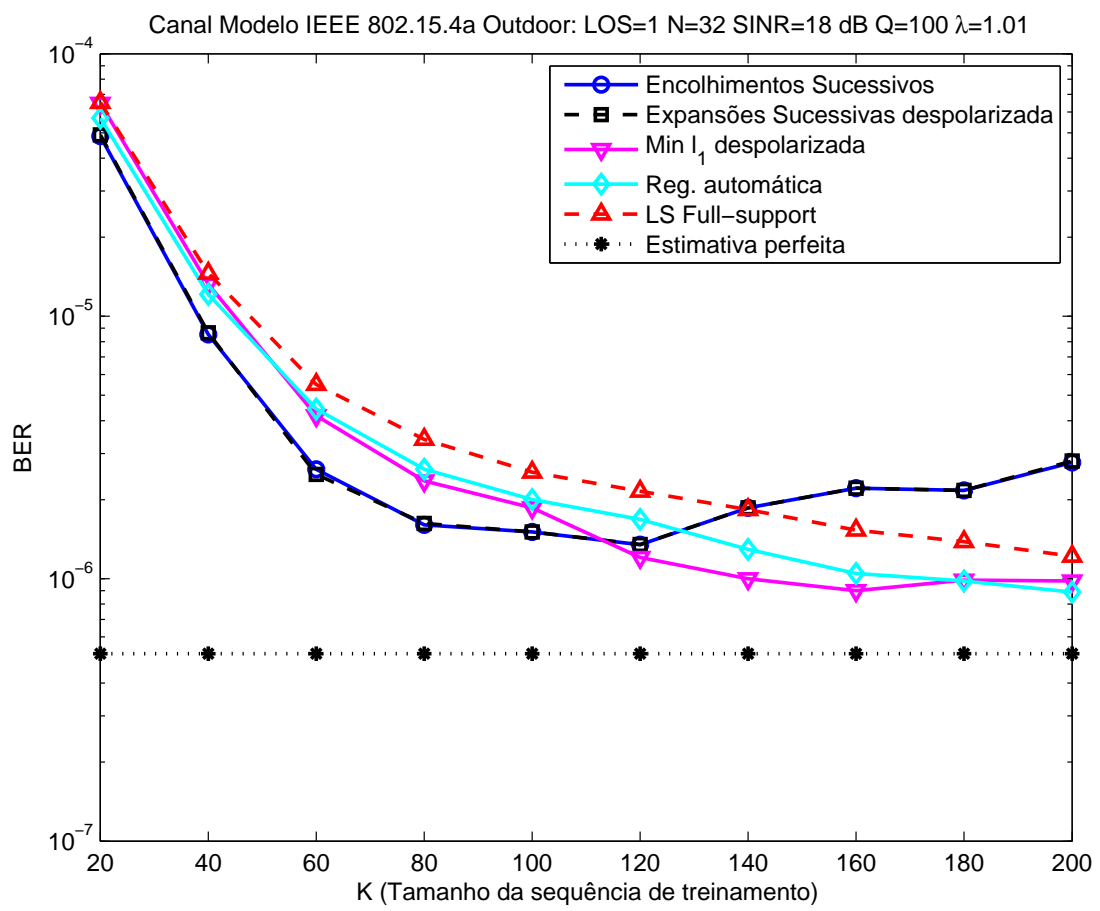

Figura 5.27: Taxa de erro de bits na estimação do canal IEEE 802.15.4a (18dB) 


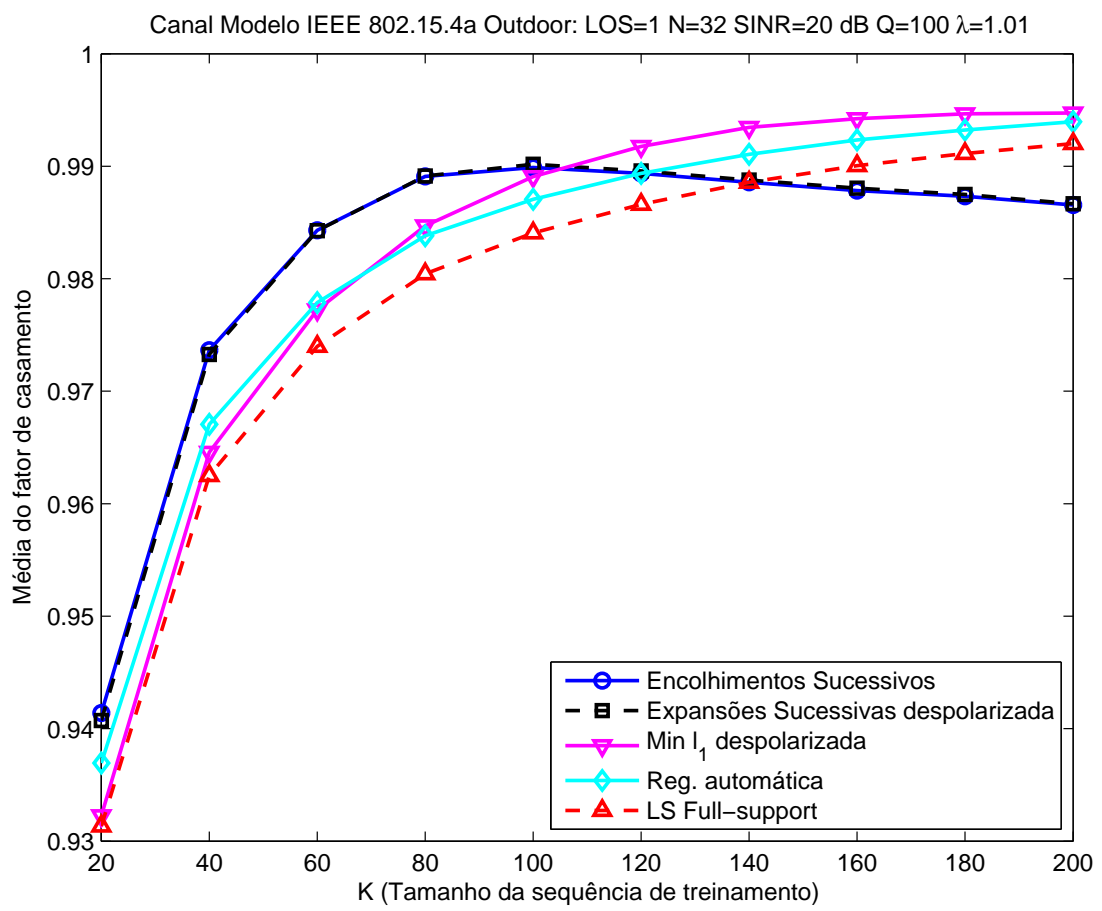

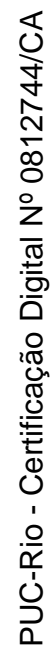

Figura 5.28: Fator de casamento na estimação do canal IEEE 802.15.4a (20dB)

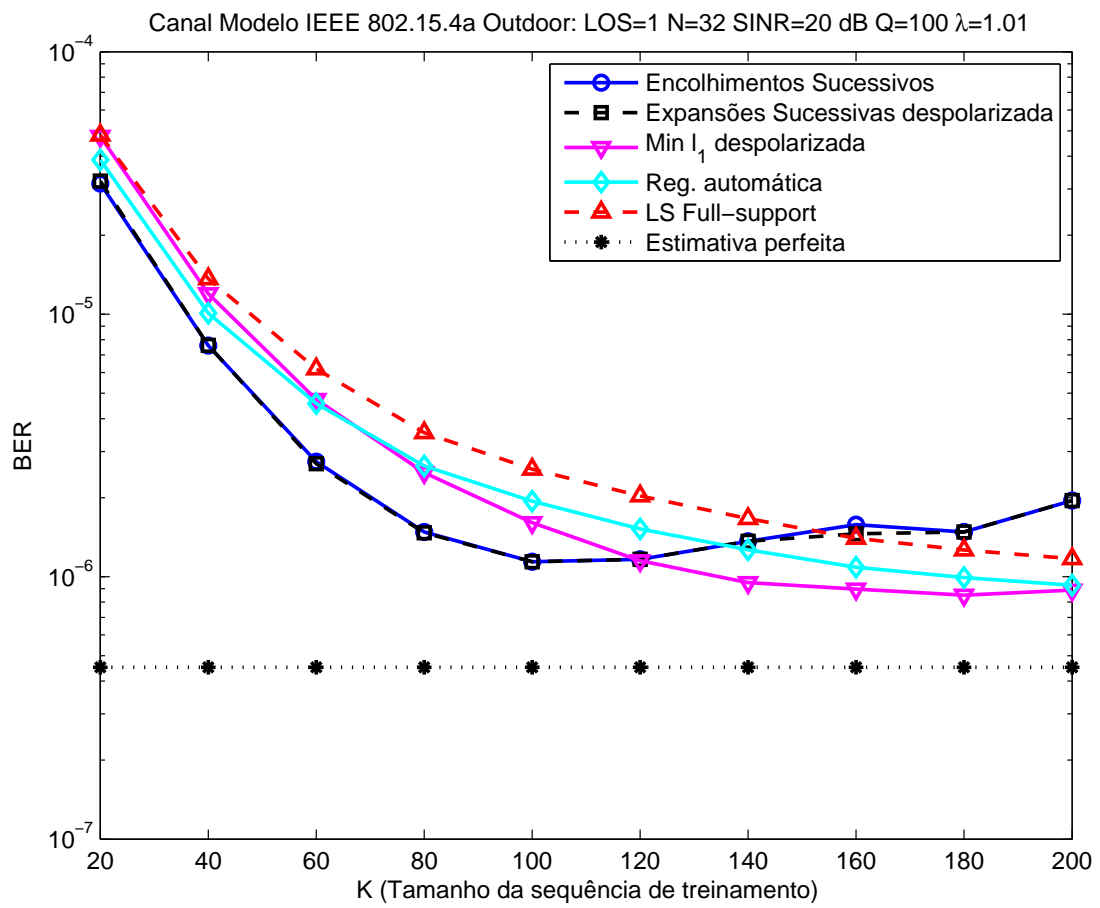

Figura 5.29: Taxa de erro de bits na estimação do canal IEEE 802.15.4a (20dB) 


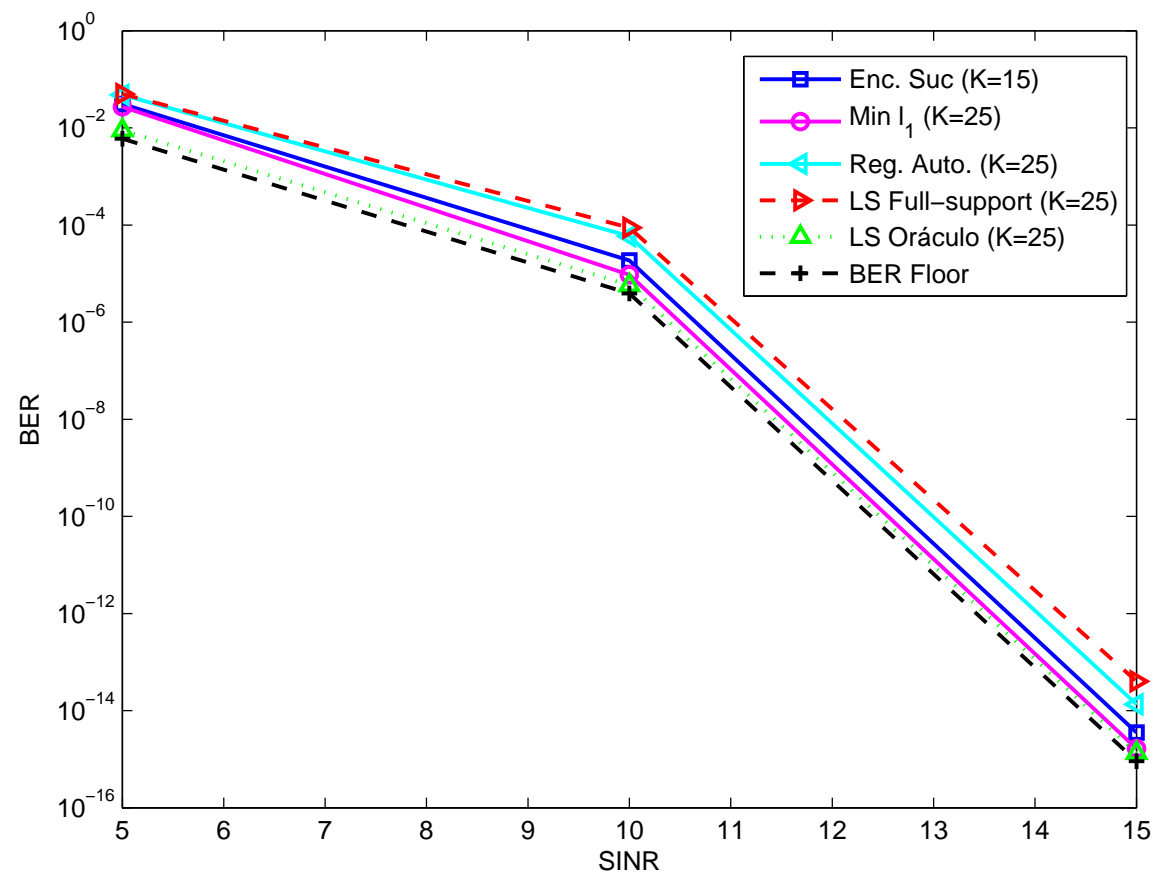

Figura 5.30: BER versus SINR

referentes à estimação de sistemas MESS, cujos resultados foram apresentados na seção 5.2. As simulações foram implementadas em Matlab e executadas um computador com Processador Intel i5M460@2,53GHz com 4GB de memória RAM. Nela, nota-se que o Método de Regularização Automática do Custo LS é o método que apresenta o maior custo computacional (contabilizando o tempo acumulado de processamento até o instante $k$ ), seguido de perto do algoritmo $l_{1}$-RLS, embora o tempo de processamento médio por iteração, representado pela linha tracejada, seja menor que o tempo de processamento apresentados pelos métodos de Encolhimentos Sucessivos e de Minimização da norma $l_{1}$ despolarizada. O algoritmo de Expansões Sucessivas despolarizada apresenta tempo de processamento bem menor que do algoritmo de Encolhimentos Sucessivos, devido ao fato de as realizações dos sistemas geradas nas simulações serem bastante esparsas ( $L=100$ e $S=10$ ). Dessa forma, o algoritmo de Expansões Sucessivas despolarizada precisa de menos iterações para produzir as estimativas que o algoritmo de Encolhimentos Sucessivos (nessas simulações em particular, aproximadamente 10 iterações contra aproximadamente 90 do algoritmo de encolhimentos sucessivos). Finalmente, é possível constatar que o algoritmo de Minimização da norma $l_{1}$ apresenta o desempenho mais estável às custas de um esforço computacional um pouco maior que o do algoritmo de Encolhimentos Sucessivos. 


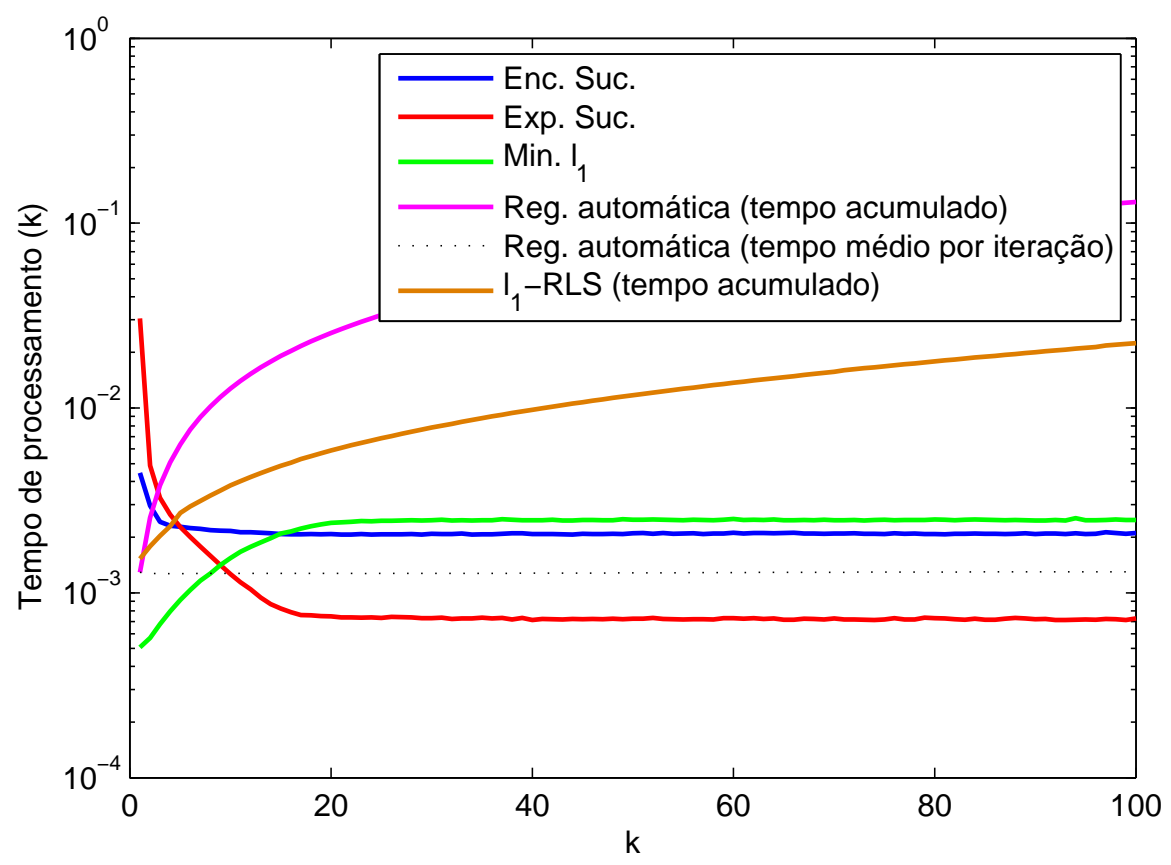

Figura 5.31: Custo computacional relativo

\section{5}

\section{Considerações finais}

Os resultados das simulações mostram que os métodos de Encolhimentos Sucessivos, Expansões Sucessivas, Minimização da norma $l_{1}$ e Ajuste automático do fator de regularização do custo LS apresentam desempenho superior que o algoritmo LS Full-support, em muitos casos alcançando o desempenho do algoritmo LS Oráculo e com a vantagem em relação ao algoritmo $l_{1}$-RLS de não apresentarem um patamar de NMSD. Além disso, os algoritmos de Encolhimentos Sucessivos, Expansões Sucessivas e Minimização da norma $l_{1}$ demandam menor esforço computacional que o algoritmo $l_{1}$-RLS. 


\section{Conclusão}

Esta Tese enfoca o problema de estimação de sistemas esparsos. A principal motivação para a elaboração deste trabalho consiste na atual proliferação de Redes de Sensores baseados na tecnologia Impulse-Radio Ultra-Wideband (IRUWB), de amplo interesse em aplicações civis e militares. A razão para isso se deve ao fato de que os modelos de canal nos quais operam os sistemas IR-UWB são tipicamente esparsos.

Os esforços envidados nesse trabalho se concentraram na elaboração de novos métodos, de baixa complexidade computacional, com a finalidade específica de estimação de sistemas esparsos. O resultado foi o desenvolvimento de quatro novos métodos para estimação de sistemas esparsos, todos baseados na técnica de estimação por mínimos quadrados, universalmente conhecida por sua denominação em inglês - Least Squares (LS) Estimation. São eles: Encolhimentos Sucessivos, Expansões Sucessivas, Minimização da norma $l_{1}$ e Ajuste automático do fator de regularização do custo LS. Objetivando acelerar a velocidade de convergência, estes novos métodos incorporam técnicas relacionadas à otimização convexa e à Teoria de Compressive Sensing. O elo de ligação entre eles decorre do fato de compartilharem o mesmo critério de relaxamento do custo LS.

Os quatro métodos de estimação de sistemas esparsos originalmente elaborados e apresentados nesta Tese foram construídos a partir do modelo de processamento em blocos da estimação LS, proposto no Capítulo 2. A generalização desse modelo possibilitou a aplicação dos métodos em questão na tarefa específica de estimação de canais esparsos em sistemas de comunicações baseados em algumas tecnologias CDMA e IR-UWB, de amplo interesse em aplicações civis e militares.

Os métodos de Encolhimentos Sucessivos, Expansões Sucessivas e Minimização da Norma $l_{1}$ intrinsecamente envolvem a estimação do suporte da resposta ao impulso em tempo discreto do sistema estimado. O método de Encolhimentos Sucessivos fundamenta-se no fato de que a sequência de mínimos custos LS, associada a uma sequência de suportes em que o suporte posterior é subconjunto do anterior, é monótona não-decrescente. O método de Expansões Sucessivas foi inspirado no algoritmo de Matching Pursuit. Esses dois algoritmos podem ser considerados antagônicos, no sentido de que o primeiro constrói uma estimativa esparsa a partir da estimativa LS full-support e promove o encolhimento do suporte da estimativa a cada iteração, enquanto que o segundo constrói a 
estimativa a partir do vetor nulo e promove iterativamente a expansão do suporte da estimativa. O método de Minimização da Norma $l_{1}$ foi inspirado nos algoritmos Basis Pursuit e LASSO e também constrói iterativamente a estimativa a partir da estimativa LS, promovendo o encolhimento do suporte a cada nova iteração. Nesse aspecto, guarda semelhança com o método de Encolhimentos Sucessivos. A diferença fundamental entre eles reside no fato de que a solução do método de minimização da norma $l_{1}$ surge do atendimento às condições de contorno de um problema de otimização convexa com restrições, enquanto que a solução do outro surge da convergência com probabilidade 1 das estimativas LS com suporte reduzido.

O método de Ajuste automático do fator de regularização do Custo LS foi inspirado nos algoritmos de regularização convexa e zero-attractor. O mérito desse algoritmo é incorporar a técnica de bisseção para a busca automática do valor ótimo do fator de regularização, que corresponde àquele que faz com que o custo LS associado à estimativa fique igual a um valor máximo admitido.

Resultados obtidos em simulações mostram que os algoritmos originalmente apresentados nesta Tese conciliam baixa complexidade computacional e excelente desempenho, em muitos casos igualando o desempenho apresentado pelo algoritmo de benchmark LS Oráculo.

Dando continuidade ao desenvolvimento de novos métodos para estimação de sistemas esparsos, uma oportunidade de pesquisa é a parametrização a partir da inserção de um fator de esquecimento, com o propósito de que esses novos métodos possam ser empregados em estimação de sistemas esparsos variantes no tempo. Outra oportunidade de pesquisa é o desenvolvimento de critérios não-empíricos para escolha do fator de relaxamento. 


\section{Referências Bibliográficas}

[1] Simon Haykin, "Adaptive Filter Theory", Prentice-Hall International, Inc., third edition, 1996 1.1, 2.1

[2] Jose L. Paredes, Gonzalo R. Arce and Zhongmin Wang, "Ultra-Wideband Compressed Sensing: Channel Estimation", IEEE Journal of Selected Topics in Signal Processing, vol. 1, no. 3, October 2007 1.1, 3.1

[3] Christian R. Berger and Zhaohui Wang, "Application of Compressive Sensing to Sparse Channel Estimation", IEEE Communications Magazine, November $20101.1,3.1$

[4] Richard G. Baraniuk, "Compressive Sensing", IEEE Signal Processing Magazine, July 20071.1

[5] , Emmanuel J. Candes and Michael B. Wakin, "An Introduction to Compressive Sampling", IEEE Signal Processing Magazine, March 20081.1

[6] S. G. Mallat and Z. Zhang, "Matching Pursuits with Time-Frequency Dictionaries", IEEE Transactions on Signal Processing, December 1993 1.1, 1.2, 4.6 .1

[7] Shane F. Cotter and Bhaskar D. Rao, "Sparse Channel Estimation via Matching Pursuit With Application to Equalization", IEEE Transactions on Communications, vol. 50, no. 3, March 20021.1

[8] Nuo Wang and Yongge Wang, "An Image Reconstruction Algorithm based on Compressed Sensing using Conjugate Gradient", IEEE IUCS, 20101.1

[9] Yih-Fang Huang and Sridhar Gollamudi, "Set-membership Identification for Adaptive Equalization", Proceedings of the 38th Midwest Symposium on Circuits and Systems, 19951.1

[10] Cecilia Carbonelli, Satish Vedantam and Urbashi Mitra, "Sparse Channel Estimation with Zero Tap Detection", IEEE Transactions on Wireless Communications, vol. 6, no. 5, May 20071.1

[11] Simon Foucart, "Hard Thresholding Pursuit: An Algorithm for Compressive Sensing", SIAM Journal on Numerical Analysis, vol. 49, Issue 6, November 2011 1.1, 1.2, 4.5.2

[12] Robert Tibshirani, "Regression Shrinkage and Selection via the Lasso", Journal of the Royal Statistical Society, Series B (Methodological), Vol. 58, No. 1, pp. 267-288, 1996 1.1, 1.2, 4.7.1 
[13] Scott Shaobing Chen, David L. Donoho and Michael A. Saunders, "Atomic Decomposition by Basis Pursuit", SIAM, Vol. 43, no. 1, pp 129-159, 2001 1.1, $1.2,4.7 .1$

[14] Md. Zulfiquar Ali Bhotto and Andreas Antoniou, "A Family of Shrinkage Adaptive-Filtering Algorithms", IEEE Transactions on Signal Processing, vol. 61, no. 7, April 20131.1

[15] Mahesh Godavarti and Alfred O. Hero, "Partial Update LMS Algorithm", IEEE Transactions on Signal Processing, vol. 53, no. 7, July 20051.1

[16] Yilun Chen, Yuantao Gu and Alfred O. Hero III, "Sparse LMS for System Identification", Proc. ICASSP, 20091.1

[17] Yuantao Gu, Jian Jin and Shunliang Mei, " $L_{0}$ Norm Constraint LMS Algorithm for Sparse System Identification", IEEE Signal Processing Letters, vol. 16, no. 9, September 2009 1.1, 1.2, 4.8.1

[18] Guan Gui, "Improved least mean square algorithm with application to adaptive sparse channel estimation", EURASIP Journal on Wireless Communications and Networking, 20131.1

[19] Masahiro Yukawa, "Krylov-Proportionate Adaptive Filtering Techniques Not Limited to Sparse Systems", IEEE Transactions omn Signal Processing, vol. 57, no. 3, March 20091.1

[20] Ender M. Eksioglu and A. Korhan Tanc, "RLS Algorithm With Convex Regularization", IEEE Signal Processing Letters, vol. 18, no. 8, August 2011 $1.1,1.2,1.3,4.8 .1,4.9,5.2$

[21] Zaiwen Wen, Wotao Yin, Donald Goldfarb and Yin Zhang, "A Fast Algorithm for Sparse Reconstruction based on Shrinkage, Subspace Optimization and Continuation", SIAM, vol. 32, no. 4, 20101.1

[22] Yuriy Zakharov and Vítor Nascimento, "Homotopy algorithm using dichotomous coordinate descent iterations for sparse recovery", Conference on Signals, Systems and Computers (ASILOMAR), 20121.1

[23] Yang, Zhaocheng; de Lamare, R.C.; Xiang Li, "Regularized STAP Algorithms With a Generalized Sidelobe Canceler Architecture for Airborne Radar", Signal Processing, IEEE Transactions on , vol.60, no.2, pp.674,686, Feb. 20121.1

[24] Panahi, A.; Viberg, M., "Fast Candidate Points Selection in the LASSO Path", Signal Processing Letters, IEEE, vol.19, no.2, pp.79,82, Feb. 20121.1 
[25] Yancheng Li, Yuantao Gu and Kun Tang, "Parallel NLMS Filters with Stochastic Active Taps and Step-sizes for Sparse System Identification", IEEE ICASP, 20061.1

[26] S. Li, R. C. de Lamare and Rui Fa, "Reduced-Rank Linear Interference Supression for DS-UWB Systems Based on Switched Approximations of Adaptive Basis Functions", IEEE Transactions on Vehicular Technology, vol. 60, no. 2, February 20111.1

[27] Andreas F. Molisch, Kannan Balakrishnan, Dajana Cassioli, Chia-Chin Chong, Shahriar Emami, Andrew Fort, Johan Karedal, Juergen Kunisch, Hans Schantz, Ulrich Schuster and Kai Siwiak, "IEEE 802.15.4a channel model - final report", 2004 1.3, 4.9, 5.1, 5.3, A

[28] Stephen Boyd and Lieven Vandenberghe, "Convex Optimization", Cambridge University Press, seventh edition, 2009 2.1, 3.7, 3.7, 4.7.2

[29] Gene H. Golub and Charles F. Van Loan, "Matrix Computations", The Johns Hopkins University Press, third edition, 1996 2.2, 2.3, 3.8

[30] Penghang Yin, Ernie Esser, and Jack Xin, "Ratio and Difference of I1 and 12 Norms and Sparse Representation with Coherent Dictionaries", Adaptive Data Analysis and Sparsity Workshop at the Institute for Pure and Applied Mathematics at UCLA, 20131

[31] Alexandre de M. Torturela, Rodrigo C. de Lamare, Cesar A. Medina and Raimundo Sampaio-Neto, "Sparse IR-UWB Channel Identification Based on Successive Relaxations and Least Squares Estimation", IEEE ISWCS-2013 2

[32] Aline de Oliveira Pinto Silva, "Detecção de Sinais em Sistema de Comunicações de Banda Ultra-Larga", Dissertação de Mestrado, Pontifícia Universidade Católica do Rio de Janeiro, Dezembro de 2010 A 
A

\section{Representação de sinais em sistemas UWB}

Uma das aplicações de particular interesse para os métodos de estimação de sistemas esparsos propostos ao longo desta Tese é a estimação de canais esparsos em sistemas de comunicações, tais quais nos sistemas UWB que seguem o padrão IEEE 802.15.4a (27). Portanto, o modelo de sinal apresentado neste anexo é apropriado para representar os sistemas de comunicações UWB que seguem o referido padrão (32).

\section{A.1}

\section{Modelo de sinal em tempo contínuo}

Seja $x(t)$ a representação em banda básica do sinal transmitido por um terminal qualquer em uma rede de comunicações, com modulação linear, dada por

$$
x(t)=\sum_{k=1}^{K} a_{k} q_{k}\left(t-k T_{q}\right)
$$

onde

- $K$ é a quantidade de símbolos transmitidos (comprimento do pacote);

- $a_{k}$ é o $k$-ésimo símbolo transmitido, com $a_{k} \in \mathbb{A}$, sendo $\mathbb{A}$ o alfabeto (conjunto de símbolos) referente ao esquema de modulação linear empregado;

- $q_{k}(t)$ é o sinal referente à sequência pseudoaleatória (código) de espalhamento direto dentro do $k$-ésimo quadro do pacote;

- $T_{q}$ é a duração de cada um dos quadros.

Considerando que o esquema de múltiplo acesso é realizado através da associação dos mecanismos de espalhamento direto por código e time-hopping, então $q_{k}(t)$ pode ser representado como

$$
q_{k}(t)=\sum_{n=1}^{N} c_{k, n} p\left(t-\Delta_{k}-(n-1) T_{c}\right)
$$

onde

- $c_{k, n}$ é o $n$-ésimo elemento do $k$-ésimo código de espalhamento direto;

- $N$ é o comprimento dos códigos de espalhamento direto; 
- $p(t)$ é o pulso básico de transmissão, com duração igual a $T_{p}$;

- $T_{c}$ é o intervalo de chip;

- $\Delta_{k}$ é o $k$-ésimo intervalo de time-hopping.

No presente trabalho, supõe-se não haver sobreposição de quadros consecutivos, ou seja,

$$
\Delta_{k} \leq \Delta_{\max } \quad \text { e } \quad \Delta_{\max }+(N-1) T_{c}+T_{p}<T_{q}
$$

O respectivo sinal recebido $y(t)$, nos terminais da antena de um usuário receptor, pode ser representado como

$$
y(t)=x(t) \star v(t)+w(t)
$$

onde o símbolo $\star$ representa o operador convolução e

- $v(t)$ é a resposta ao impulso do canal, considerado invariante ao longo da duração do pacote;

- $w(t)$ é processo estocástico correspondente ao ruído mais interferência.

Admitindo que o filtro receptor seja casado ao pulso básico de transmissão, o sinal na saída do filtro casado pode ser representado como

$$
r(t)=y(t) \star p(-t)
$$

Utilizando A-2 e A-4 em A-5, o sinal $r(t)$ pode ser reescrito como

$$
r(t)=\sum_{k=1}^{K} a_{k}\left[\sum_{n=1}^{N} c_{k, n} h\left(t-k T_{q}-\Delta_{k}-(n-1) T_{c}\right)\right]+z(t)
$$

onde

$$
h(t)=p(t) \star v(t) \star p\left(T_{p}-t\right)
$$

é a resposta ao impulso do canal equivalente e

$$
z(t)=w(t) \star p\left(T_{p}-t\right)
$$

é o ruído-mais-interferência equivalente.

A equação A-6 pode ser reescrita como

$$
r(t)=\sum_{k=1}^{K} a_{k} s_{k}\left(t-k T_{q}-\Delta_{k}\right)+z_{k}(t)
$$


onde

$$
s_{k}(t)=\sum_{n=1}^{N} c_{k, n} h\left(t-(n-1) T_{c}\right)
$$

é a assinatura efetiva associada ao $k$-ésimo quadro.

\section{A.2}

\section{Modelo de sinal em tempo discreto}

Para fins de detecção coerente de sinais, o sinal na saída do filtro de recepção $r(t)$ deve ser devidamente sincronizado e amostrado. Seja $r_{k, m}$ a amostra de $r(t)$ no instante $t_{k, m}$, dado por

$$
r_{k, m}=r\left(t_{k, m}\right) \mid t_{k, m}=k T_{q}+\Delta_{k}+(m-1) T_{c}
$$

Aplicando A-9 em A-11, verifica-se que

$$
r_{k, m}=a_{k} s_{k, m}+z_{k, m}+\tilde{s}_{k, m}
$$

onde

$$
s_{k, m}=s_{k}\left((m-1) T_{c}\right)=\sum_{n=1}^{N} c_{k, n} h\left((m-n) T_{c}\right)
$$

é a $m$-ésima amostra referente à $k$-ésima assinatura efetiva,

$$
z_{k, m}=z\left(t_{k, m}\right)
$$

é a amostra de $z(t)$ no instante $t_{k, m} \mathrm{e}$

$$
\tilde{s}_{k, m}=\sum_{i \neq k} a_{i} s_{i}\left(t_{k, m}-i T_{q}-\Delta_{i}\right)
$$

é a amostra no instante $t_{k, m}$ do sinal referente à interferência provocada pela sobreposição das assinaturas efetivas anteriores e posteriores à $k$-ésima assinatura efetiva.

Seja $T_{h}$ a duração de $h(t)$. Observando A-10, verifica-se que a duração $T_{s}$ de cada uma das assinaturas efetivas é dada por

$$
T_{s}=T_{h}+(N-1) T_{c}
$$

No presente trabalho, supõe-se que $h(t)=0 t<0$ e que

$$
T_{s}+\Delta_{\max }<T_{q}
$$


Dessa forma, são evitadas interferências entre assinaturas efetivas consecutivas e, com isso, tem-se que

$$
\tilde{s}_{k, m}=0 \quad \forall k, m
$$

A quantidade necessária de amostras de $r(t)$, para fins de detecção de $a_{k}$ com estatística suficiente, é dada por

$$
M=\left\lceil\frac{T_{s}}{T_{c}}\right\rceil
$$

ou equivalentemente

$$
M=N+L-1
$$

onde

$$
L=\left\lceil\frac{T_{h}}{T_{c}}\right\rceil
$$

Seja $\mathbf{r}_{k}=\left[\begin{array}{llll}r_{k, 1} & r_{k, 2} & \ldots & r_{k, M}\end{array}\right]^{T} \mathrm{o} k$-ésimo vetor de observação, de dimensão $M \times 1$. Observando A-12 e supondo que a condição em A-18 é satisfeita, verifica-se que $\mathbf{r}_{k}$ pode ser expresso na seguinte notação vetorial

$$
\mathbf{r}_{k}=a_{k} \mathbf{s}_{k}+\mathbf{z}_{k}
$$

onde $\mathbf{s}_{k}=\left[\begin{array}{llll}s_{k, 1} & s_{k, 2} & \ldots & s_{k, M}\end{array}\right]^{T}$ é a representação discreta da assinatura efetiva e $\mathbf{z}_{k}=\left[\begin{array}{llll}z_{k, 1} & z_{k, 2} & \ldots & z_{k, M}\end{array}\right]^{T}$ é a representação discreta do sinal correspondente ao ruído mais interferência.

A equação A-13 pode ser reescrita da seguinte forma

$$
\mathbf{s}_{k}=\mathbf{c}_{k} \star \mathbf{h}
$$

onde $\star$ representa o operador convolução discreta e

- $\mathbf{c}_{k}=\left[\begin{array}{llll}c_{k, 1} & c_{k, 2} & \ldots & c_{k, N}\end{array}\right]^{T}$ é vetor de dimensão $N \times 1$ que representa o $k$-ésimo código pseudoaleatório de espalhamento direto;

$-\mathbf{h}=\left[h(0) h\left(T_{c}\right) h\left(2 T_{c}\right) \ldots h\left((L-1) T_{c}\right)\right]^{T}$ é vetor de dimensão $L \times 1$ que representa a resposta ao impulso discreta do canal equivalente $h(t)$.

A equação A-23 também pode ser expressa na seguinte notação algébrica

$$
\mathbf{s}_{k}=\mathbf{C}_{k} \mathbf{h}
$$




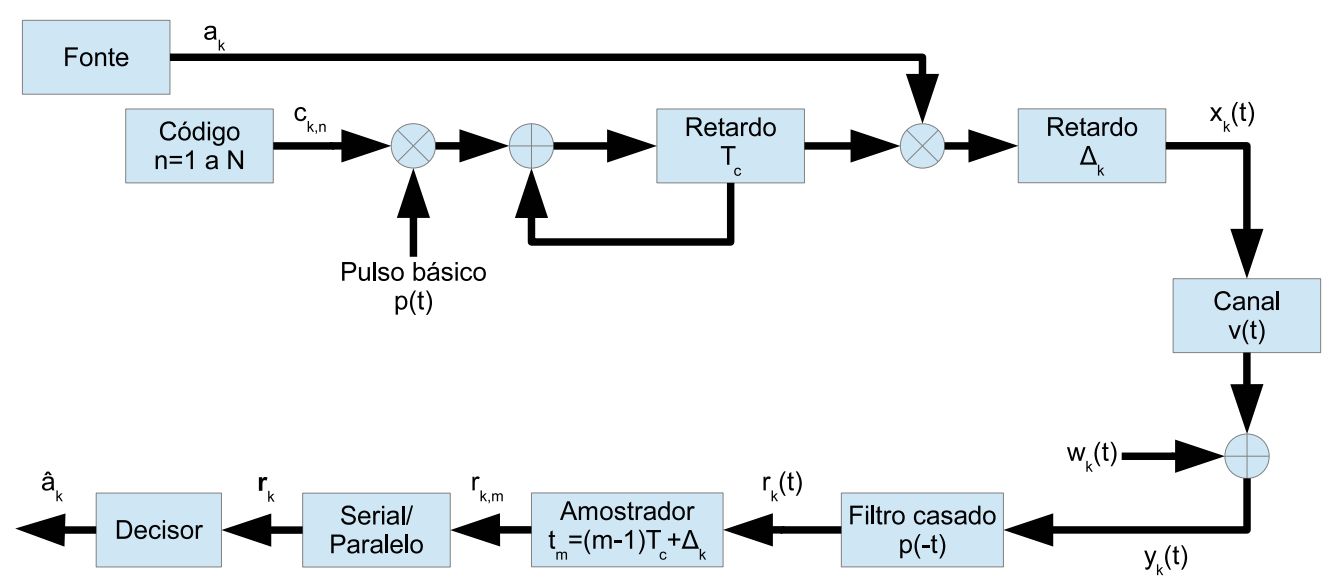

Figura A.1: Diagrama de um Sistema de Comunicações com Espalhamento Direto por Código e Time-Hopping

onde $\mathbf{C}_{k}$ é matriz Toeplitz cujo produto $\mathbf{C}_{k} \mathbf{h}$ é igual à convolução discreta entre $\mathbf{c}_{k}$ e $\mathbf{h}$.

Portanto, utilizando A-23, é possível reescrever A-22 como

$$
\mathbf{r}_{k}=a_{k} \mathbf{c}_{k} \star \mathbf{h}+\mathbf{z}_{k}
$$

ou ainda, utilizando A-24, é possível reescrever A-22 como

$$
\mathbf{r}_{k}=a_{k} \mathbf{C}_{k} \mathbf{h}+\mathbf{z}_{k}
$$

A Figura A.1 mostra o modelo de sistema de comunicações adotado. A Figura A.2 mostra o modelo equivalente ao apresentado na Figura A.1.

O modelo discreto ora apresentado será empregado, de forma genérica ao longo desta Tese, como o esquema em que os métodos propostos para estimação de sistemas esparsos são aplicados.

\section{A.3}

\section{Regras de decisão de símbolos}

Seja $\hat{a}_{k}$ o $k$-ésimo símbolo decidido utilizando o critério de decisão de Máxima Probabilidade a Posteriori (critério MAP), expresso como

$$
\hat{a}_{k}=\arg \max _{a \in \mathbb{A}} P\left(a_{k}=a \mid \mathbf{r}_{k}\right)
$$

Pela regra de Bayes, a probabilidade a posteriori $P\left(a \mid \mathbf{r}_{k}\right)$ pode ser expressa em função das probabilidades e densidades de probabilidade a priori da seguinte forma 


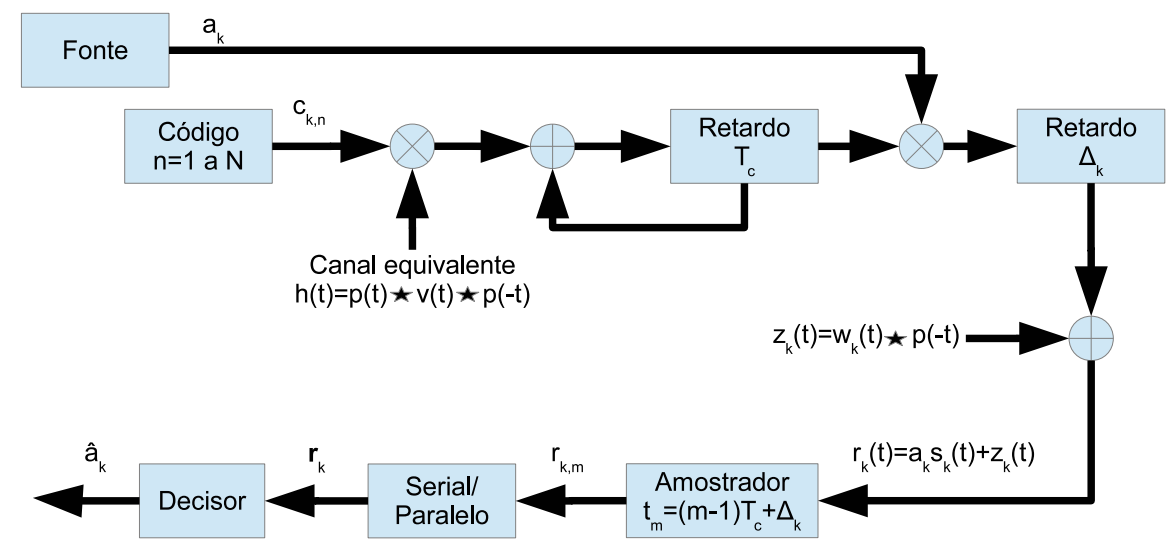

Figura A.2: Diagrama equivalente do Sistema de Comunicações representado na Fig. A.1

$$
P\left(a_{k}=a \mid \mathbf{r}_{k}\right)=\frac{P\left(a_{k}=a\right) p_{\mathbf{r}}\left(\mathbf{r}_{k} \mid a_{k}=a\right)}{p_{\mathbf{r}}\left(\mathbf{r}_{k}\right)}
$$

onde $p_{\mathbf{r}}($.$) é função densidade de probabilidade do vetor de observação.$

Se os símbolos $a \in \mathbb{A}$ forem equiprováveis, então aplicando A-28 em A-27, o critério MAP pode ser substituído pelo critério de Máxima Verossimilhança (critério ML), dado por

$$
\hat{a}_{k}=\arg \max _{a \in \mathbb{A}} p\left(\mathbf{r}_{k} \mid a_{k}=a\right)
$$

Utilizando A-22, o vetor $\mathbf{z}_{k}$ (que representa o ruído mais interferência) pode ser expresso como

$$
\mathbf{z}_{k}=\mathbf{r}_{k}-a_{k} \mathbf{s}_{k}
$$

Suponha que $w(t)$ seja processo estacionário branco com média zero e função autocorrelação $\sigma_{w}^{2} \delta(\tau)$, e que $p(t)$ atende ao critério de Nyquist para eliminação de interferência entre símbolos. Considere ainda, sem perda de generalidade, que a energia de $p(t)$ seja igual a 1 . Supondo sincronização perfeita entre transmissor e receptor e observando A-8, verifica-se que $\mathbf{z}_{k}$ é vetor gaussiano complexo com média $\mathbf{0}$ e matriz de autocorrelação $\sigma_{w}^{2} \mathbf{I}$. Com isso, a função densidade de probabilidade a priori $p_{\mathbf{r}}\left(\mathbf{r}_{k} \mid a_{k}=a\right)$ pode ser expressa como

$$
p_{\mathbf{r}}\left(\mathbf{r}_{k} \mid a_{k}=a\right)=p_{\mathbf{z}_{k}}\left(\mathbf{r}_{k}-a \mathbf{s}_{k}\right)=\frac{1}{(2 \pi)^{M}} \exp \left[-\frac{1}{\sigma_{w}^{2}}\left\|\mathbf{r}_{k}-a \mathbf{s}_{k}\right\|^{2}\right]
$$

Observando A-31, verifica-se que, no caso particular em que $\mathbf{z}_{k}$ é vetor gaussiano complexo com média $\mathbf{0}$ e matriz de autocorrelação $\sigma_{w}^{2} \mathbf{I}$, o critério de 
decisão expresso em A-29 pode ser expresso pela regra de decisão pela mínima distância, dada por

$$
\hat{a}_{k}=\arg \min _{a \in \mathbb{A}}\left\|\mathbf{r}_{k}-a \mathbf{s}_{k}\right\|^{2}
$$

No caso específico de modulações em fase, nas quais $\left|a_{k}\right|$ é uma constante (isto é, $\left|a_{k}\right|^{2}=\mathcal{E}_{a} \forall a_{k} \in \mathbb{A}$ ), a regra de decisão em A-32 pode ser substituída pela regra de decisão pela máxima correlação entre o sinal reconstituído $a \mathbf{s}_{k}$, (com $a \in \mathbb{A}$ ) e o vetor de observação $\mathbf{r}_{k}$, dada por

$$
\hat{a}_{k}=\arg \max _{a \in \mathbb{A}} \Re\left[a^{*} \mathbf{s}_{k}^{H} \mathbf{r}_{k}\right]
$$

No caso ainda mais particular em que $\mathbb{A}=\{-1,+1\}$ (que corresponde ao esquema de modulação binária antipodal), a regra de decisão em A-33 pode ser simplificada da seguinte forma

$$
\hat{a}_{k} \stackrel{+1}{\gtrless} \Re\left[\mathbf{s}_{k}^{H} \mathbf{r}_{k}\right]
$$

\section{A.4 \\ Impacto do erro de estimação do canal na detecção}

As regras de decisão de símbolos apresentadas na seção anterior fazem uso da assinatura efetiva. Entretanto, para a assinatura efetiva ser calculada, é necessário conhecer a resposta ao impulso do canal equivalente discreto. Porém, na prática, o receptor dispõe apenas de uma estimativa da resposta ao impulso do canal. Esta seção apresenta uma análise do impacto provocado pelo erro de estimação da resposta ao impulso do canal equivalente discreto na degradação da taxa de erro de símbolos (ou de bits), particularmente para os esquemas de modulação BPSK e bi-ortogonal, empregados no padrão UWB IEEE 802.15.4a.

Seja $\hat{\mathbf{s}}_{k}$ a estimativa da $k$-ésima assinatura efetiva, dada por

$$
\hat{\mathbf{s}}_{k}=\mathbf{C}_{k} \hat{\mathbf{h}}
$$

onde $\hat{\mathbf{h}}$ é a estimativa da resposta ao impulso do canal equivalente discreto.

Perante o desconhecimento da resposta ao impulso do canal equivalente discreto e no caso específico de modulação BPSK, em que o alfabeto é $\mathbb{A}=\{-1,+1\}$, o critério de decisão em A-34 deve ser substituído pelo seguinte critério de decisão

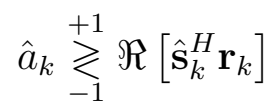


Utilizando A-22, o critério de decisão em A-36 pode ser expandido como

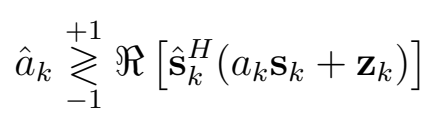

que, por sua vez, pode ser reescrito como

$$
\hat{a}_{k} \stackrel{+1}{\gtrless} \operatorname{sgn}\left[\Re\left(\hat{\mathbf{s}}_{k}^{H} \mathbf{s}_{k}\right)\right] a_{k} \sqrt{\mathcal{E}_{b}}+n
$$

em que $\mathcal{E}_{b}$ é a energia do bit na entrada do módulo decisor, dada por

$$
\mathcal{E}_{b}=\left[\Re\left(\hat{\mathbf{s}}_{k}^{H} \mathbf{s}_{k}\right)\right]^{2}
$$

$\operatorname{sgn}():. \mathbb{R}^{*} \rightarrow\{-1,+1\}$ é a função-sinal, definida como

$$
\operatorname{sgn}(x) \triangleq \frac{x}{|x|}
$$

e $n$ é variável aleatória dada por

$$
n=\Re\left[\hat{\mathbf{s}}_{k} \mathbf{z}_{k}\right]
$$

Suponha que os códigos $\mathbf{c}_{k}$ sejam projetados de forma que

$$
\mathbf{C}_{k}^{H} \mathbf{C}_{k}=\mathcal{E}_{c} \mathbf{I} \quad \forall k
$$

em que $\mathcal{E}_{c}=N \mathcal{E}_{\text {chip }}$ é a energia dos códigos de espalhamento.

Utilizando A-35 e A-42, A-39 pode ser reescrita como

$$
\mathcal{E}_{b}=\mathcal{E}_{c}^{2}\left[\Re\left(\hat{\mathbf{h}}^{H} \mathbf{h}\right)\right]^{2}
$$

Por sua vez, supondo que $\mathbf{z}_{k}$ seja vetor gaussiano de média zero e matrizautocorrelação $\sigma_{z}^{2} \mathbf{I}$, tem-se que $n$ é variável aleatória gaussiana de média zero e variância $\sigma_{n}^{2}$, dada por

$$
\sigma_{n}^{2}=\frac{1}{2} E\left[\left|\hat{\mathbf{s}}_{k}^{H} \mathbf{z}_{k}\right|^{2}\right]
$$

Utilizando A-35 e A-42, A-44 pode ser reescrita como

$$
\sigma_{n}^{2}=\frac{\mathcal{E}_{c} \sigma_{z}^{2}}{2}\|\hat{\mathbf{h}}\|^{2}
$$

Portanto, a razão sinal-ruído-mais-interferência na entrada do decisor é

$$
\frac{\mathcal{E}_{b}}{\sigma_{n}^{2}}=\frac{2 \mathcal{E}_{c}}{\sigma_{z}^{2}} \frac{\left[\Re\left(\hat{\mathbf{h}}^{H} \mathbf{h}\right)\right]^{2}}{\|\hat{\mathbf{h}}\|^{2}}
$$


A razão sinal-ruído-mais-interferência média na entrada do filtro de recepção é definida como

$$
\mathrm{SINR} \triangleq \frac{\mathcal{E}_{c} E\left[\|\mathbf{h}\|^{2}\right]}{\sigma_{z}^{2}}
$$

Empregando A-47, A-46 pode ser reescrita como

$$
\frac{\mathcal{E}_{b}}{\sigma_{n}^{2}}=\frac{2 \Theta^{2}\|\mathbf{h}\|^{2}}{E\left[\|\mathbf{h}\|^{2}\right]} \mathrm{SINR}
$$

onde $\Theta$ é o fator de casamento entre $\hat{\mathbf{h}}$ e $\mathbf{h}$, definido como

$$
\Theta \triangleq \frac{\Re\left[\hat{\mathbf{h}}^{H} \mathbf{h}\right]}{\|\hat{\mathbf{h}}\|\|\mathbf{h}\|}
$$

Utilizando a Desigualdade de Cauchy-Schwarz, é possível mostrar que

$$
-1 \leq \Theta \leq 1
$$

com $\Theta=1$ se e somente se $\hat{\mathbf{h}}=\kappa \mathbf{h}$, com $\kappa$ real positivo.

Utilizando A-49 e A-42, é possível reescrever A-38 como

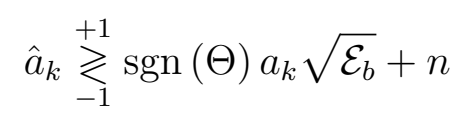

A taxa de erro de bits (Bit Error Rate - BER) para modulação BPSK, tendo como critério de decisão a regra estabelecida em A-51, é dada por

$$
\operatorname{BER}_{2}\left(\frac{\mathcal{E}_{b}}{\sigma_{n}^{2}}, \Theta\right)=\left\{\begin{array}{cc}
Q\left(\sqrt{\frac{\mathcal{E}_{b}}{\sigma_{n}^{2}}}\right) & \Theta \geq 0 \\
1-Q\left(\sqrt{\frac{\mathcal{E}_{b}}{\sigma_{n}^{2}}}\right) & \Theta<0
\end{array}\right.
$$

na qual a função $Q($.$) é definida como$

$$
Q(x) \triangleq \frac{1}{\sqrt{2 \pi}} \int_{x}^{\infty} e^{-\frac{Y^{2}}{2}} d Y
$$

Finalmente, empregando A-48 em A-52, a taxa de erro de bits da modulação BPSK, em função de SINR, $E\left[\|\mathbf{h}\|^{2}\right]$, h e $\Theta$ pode ser expressa como

$$
\mathrm{BER}_{2}\left(\operatorname{SINR}, E\left[\|\mathbf{h}\|^{2}\right], \mathbf{h}, \Theta\right)=\left\{\begin{array}{cc}
Q\left(\sqrt{\frac{2 \Theta^{2}\|\mathbf{h}\|^{2}}{E\left[\|\mathbf{h}\|^{2}\right]} \mathrm{SINR}}\right) & \Theta \geq 0 \\
1-Q\left(\sqrt{\frac{2 \Theta^{2}\|\mathbf{h}\|^{2}}{E\left[\|\mathbf{h}\|^{2}\right]} \mathrm{SINR}}\right) & \Theta<0
\end{array}\right.
$$


Como $Q($.$) é função monótona decrescente, conclui-se que, para um valor de$ SINR qualquer, quanto menor $\Theta$, maior a taxa de erro de bits.

No caso de sistemas com modulação bi-ortogonal quaternário, tais quais os sistemas que seguem o padrão IEEE 802.15.4a, o detector pode ser visto como dois bancos de decisores BPSK paralelos, nos quais decide-se pelo símbolo que apresenta a maior correlação dentre os dois bancos. Portanto, a taxa de erro de bits dos sistemas com modulação bi-ortogonal é a mesma do esquema de modulação QPSK e pode ser expressa como

$$
\mathrm{BER}_{4}\left(\operatorname{SINR}, E\left[\|\mathbf{h}\|^{2}\right], \mathbf{h}, \Theta\right)=\left\{\begin{array}{cc}
b_{4}\left(\frac{2 \Theta^{2}\|\mathbf{h}\|^{2}}{E\left[\|\mathbf{h}\|^{2}\right]} \mathrm{SINR}\right) & \Theta \geq 0 \\
1-b_{4}\left(\frac{2 \Theta^{2}\|\mathbf{h}\|^{2}}{E\left[\|\mathbf{h}\|^{2}\right]} \mathrm{SINR}\right) & \Theta<0
\end{array}\right.
$$

em que

$$
b_{4}(x)=2 Q(\sqrt{x})-Q^{2}(\sqrt{x})
$$

Um dos objetivos das estratégias de identificação de sistemas esparsos apresentadas ao longo deste trabalho é, quando aplicadas em sistemas de comunicações na estimação de canais esparsos, reduzir a taxa de erro de bits, a partir da geração de estimativas $\hat{\mathbf{h}}$ da resposta ao impulso do canal com o maior fator de casamento possível com a resposta ao impulso verdadeira $\mathbf{h}$, empregando a menor sequência de treinamento possível. 


\section{B}

\section{O Sistema Esparso de Máxima Entropia}

Seja h o vetor-coluna que representa uma realização de um Sistema Esparso de Máxima Entropia (Maximum Entropy Sparse System - MESS). Ao longo deste trabalho, o MESS é representado pela notação $\operatorname{MESS}(\mathrm{L}, \mathrm{S})$, cujos parâmetros são:

- o comprimento $L$ do vetor $\mathbf{h}$;

- a quantidade $S$ de elementos não-nulos em h.

Seja $\mathbb{L}=\{1,2, \ldots, L\}$. Cada realização $\mathbf{h}$ do MESS é gerada da seguinte forma:

1. São sorteados $S$ elementos distintos de $\mathbb{L}$, com todos os elementos de $\mathbb{L}$ equiprováveis e estatisticamente independentes entre si. Esses $S$ elementos formam o suporte $\mathbb{S}_{\mathbf{h}}$ da realização $\mathbf{h}$, isto é, o conjunto dos $S$ elementos não-nulos de $\mathbf{h}$.

2. Os elementos cujos índices pertencem a $\mathbb{S}_{\mathbf{h}}$ são sorteados. Esses elementos consistem em variáveis aleatórias gaussianas complexas independentes entre si, de média zero e mesma variância igual a $1 / S$.

3. É atribuído o valor zero aos elementos de $\mathbf{h}$ cujos índices não pertencem a $\mathbb{S}_{\mathrm{h}}$

Todos os possíveis suportes, que correspondem a todas as possibilidades de subconjuntos de $\mathbb{L}$ com cardinalidade igual a $S$, são amostras de uma variável aleatória discreta com distribuição uniforme, o que corresponde à distribuição discreta de máxima entropia. Os elementos não-nulos de $\mathbf{h}$ formam um vetor gaussiano com média zero e matriz autocorrelação diagonal, o que corresponde à função densidade de probabilidade conjunta de máxima entropia. Por essas duas razões, o presente modelo é aqui denominado Sistema Esparso de Máxima Entropia (MESS). Nesse sentido, o MESS apresenta máxima variabilidade e, por isso, constitui-se em um excelente modelo de referência para testar a eficiência dos algoritmos de estimação de sistemas esparsos propostos ao longo desta Tese. 